\title{
EFFECTS OF HEAT STRESS AND FESCUE TOXICOSIS ON THE IMMUNE SYSTEM AND OTHER PHYSIOLOGICAL PARAMETERS
}

\author{
A Thesis presented to \\ the faculty of the graduate school \\ at the University of Missouri - Columbia
}

In Partial Fulfillment of the

Requirements for the Degree

Master of Science

by

Deepan K. Kishore

Dr. Donald Spiers, Thesis Supervisor

DECEMBER 2010 
The undersigned, appointed by the Dean of the Graduate School, have examined the Thesis entitled

\title{
EFFECTS OF HEAT STRESS AND FESCUE TOXICOSIS ON THE IMMUNE SYSTEM AND OTHER PHYSIOLOGICAL PARAMETERS
}

Presented by Deepan Kirubaharan Kishore

A candidate for the degree of Master of Science

We hereby certify that, in their opinion, it is worthy of acceptance.

\section{Dr. Donald Spiers - Thesis Advisor}

\author{
Dr. Kevin Fritsche
}

Dr. Matthew Waldron

Dr. Tim Evans 


\section{ACKNOWLEDGEMENTS}

I thank my advisor Dr Spiers, for giving me an opportunity to work in the area of fescue toxicosis and heat stress. He has helped and guided me in all aspects of my life since since I arrived in the United States. I am grateful to him for his continous encouragement and suggestions while I was learning new techniques, and analyzing voluminous amount of data. He has also spent a great amount of time in teaching me to present my results and develop a coherent writing style. A special thanks to Dr Fritsche for teaching me techniques in immunology and graciously allowing me to use his laboratory. I am glad that I was able to meet him any time I needed help and I thank him for his invaluable suggestions whenever I approached him. I thank Dr Waldron and Dr Evans for their guidance and support during my thesis project. I am grateful to Dr Evans and Dr Murphy for helping me pursue my interest in Veterinary medicine.

Peggy Ann has been very helpful in setting up all my experiments and I appreciate her patience in guiding me in the analysis of all of my data. I thank all the graduate students in our lab and it has been a wonderful and memorable experience working with Brad, Eric, Jay, Hilary, Jenny and Roxanne. I also thank Stephanie, Sara, Nathan, Wei and Laura for helping me during my thesis projects. I thank all the graduate students in ASRC for making my stay in Missouri a memorable one. A special thanks to Karthick, Vinod, Kalai, Chandrasekar, Thanga, Sudhan, Rohan, Saurav, Aravind, Gopi, Ganesh and all my other friends for their support and encouragement.

I thank my parents, Kirubaharan and Shoba, grandparents, Arul Selvaraj and 
Navaratna for their prayers, blessings and support during my Masters degree program.

I dedicate this thesis to my late Grandma Kamala and I thank her for all that she has done for me. 


\section{TABLE OF CONTENTS}

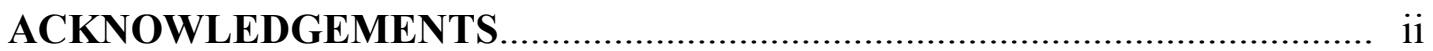

LIST OF FIGURES ................................................... viii

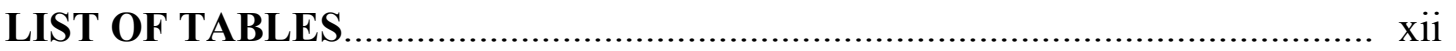

\section{CHAPTER}

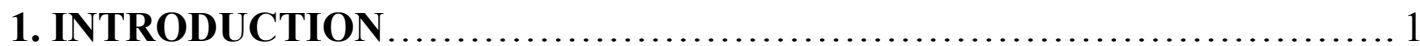

\section{LITERATURE REVIEW}

1. FESCUE TOXICOSIS ..................................................

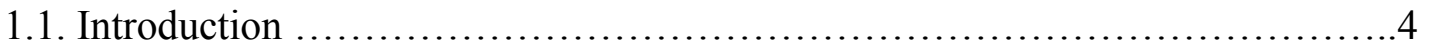

1.2. Toxins in tall Fescue grass........................................ 4

1.3. Fescue toxicosis and thermoregulation $\ldots \ldots \ldots \ldots \ldots \ldots \ldots \ldots \ldots \ldots \ldots \ldots \ldots$

1.4. Fescue toxicosis and feed inake ........................................ 6

1.5. Effects of fescue toxicosis on bodyweight and growth rate ................... 7

1.6. Effects fescue toxicosis on prolactin................................... 8

1.7. Fescue toxicosis and immune system.................................. 8

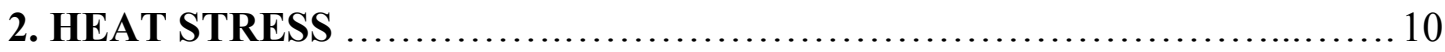

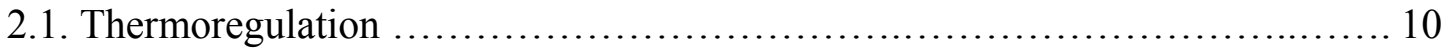

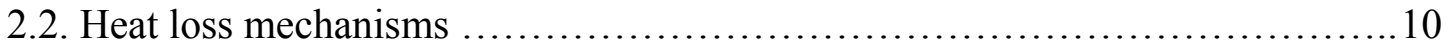

2.3. Heat stress and immune system......................................... 12

2.4. Heat stress and effects on production parameters......................... 12

3. CALORIC RESTRICTION ............................................. 13

3.1. Caloric restriction and immune system................................ 13

3.2. Caloric restriction and core body temperature............................ 13

4. INTERACTION BETWEEN STRESSORS .......................... 14

4.1. Effects of heat stress and fescue toxicosis on feed intake .................. 14

4.2. Effects of heat stress and fescue toxicosis on core body temperature .......... 15

4.3. Effects of stressors on gross motor activity .............................. 15 
5.1. Immune system overview........................................... 16

5.2. Lipopolysaccharide administration..................................... 22

5.2.1. Introduction........................................................ 22

5.2.2. Mechanism of action ................................................. 22

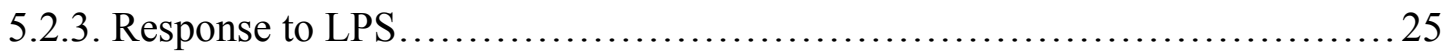

5. 2.4. LPS and Core body temperature...................................... 28

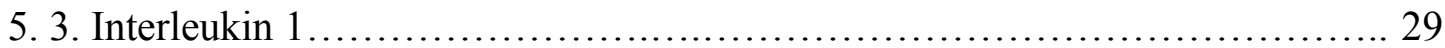

5. 4. Tumor necrosis factor alpha........................................... 29

5. 5. Lymphocytes........................................................... 30

5. 5.1. T-lymphocyte differentiation and maturation.......................... 30

5. 5.2. B-lymphocyte overview ................................................ 33

5. 5.3. Natural killer cell overview.......................................... 33

\section{LACK OF ADAPTATION TO FESCUE TOXICOSIS UNDER THERMONEUTRAL AND HEAT STRESS CONDITIONS}

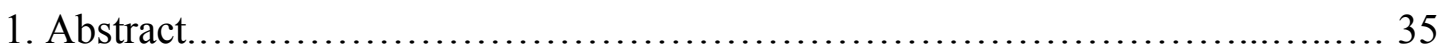

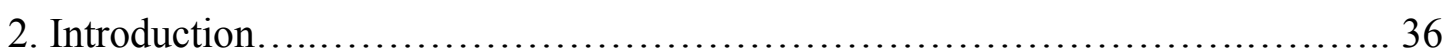

3. Materials and Methods ....................................................... 37

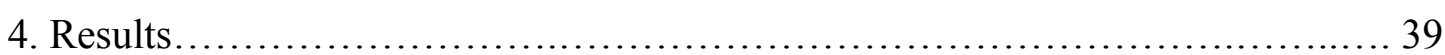

5. Discussion................................................................. 43

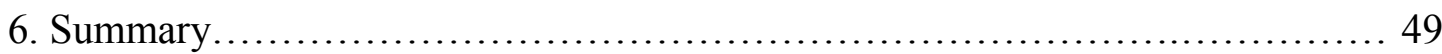

IV. EFFECT OF HEAT STRESS AND ERGOPEPTINE ALKALOIDS ON THE IMMUNE SYSTEM OF RATS

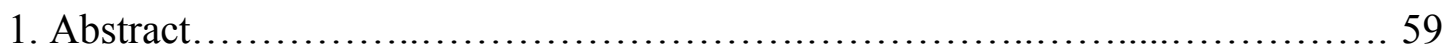

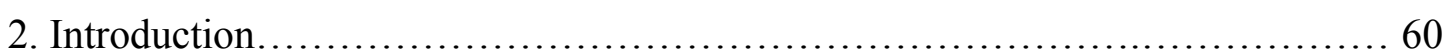

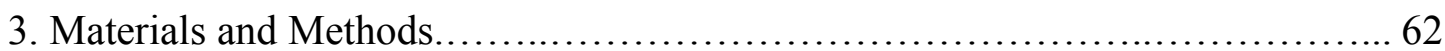

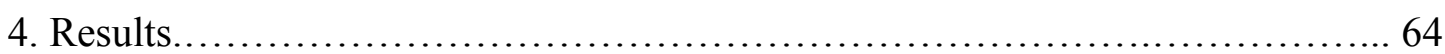

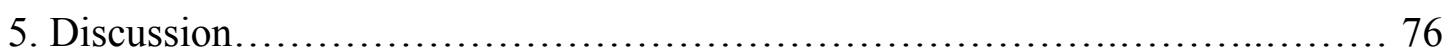

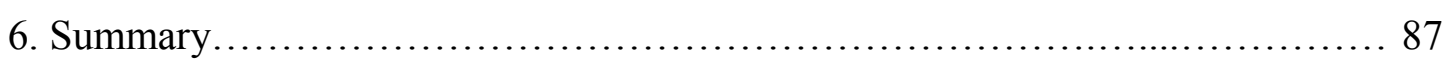

V. EFFECT OF SUBCHRONIC HEAT STRESS AND ERGOPEPTINE ALKALOIDS ON THE IMMUNE SYSTEM OF RATS

1. Abstract................................................................... 110

2. Introduction........................................................ 111 


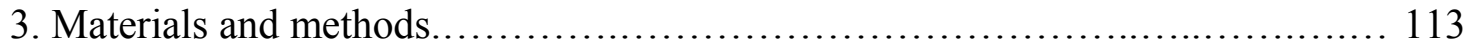

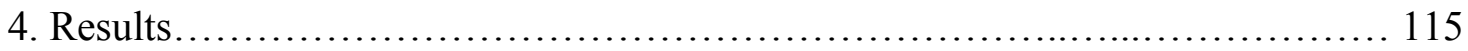

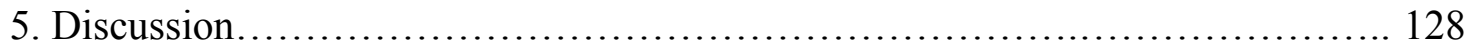

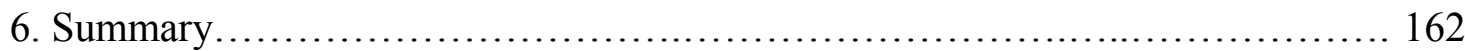

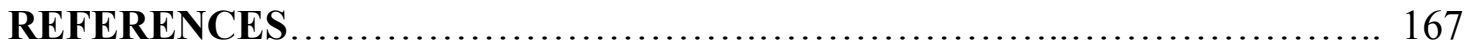




\section{APPENDICES}

Appendix 1. Implanting mini-mitters in rats................................ 181

Appendix 2. Flow cytometry................................................. 182

Appendix 3. Total numbers............................................... 186

Appendix 4. Flow cytometry results......................................... 187 


\section{LIST OF FIGURES}

Figure

1. Innate immune response and immune cells associated with the process of inflammation

1.1. Shows association of TRL4 to LPS, followed by a downward signalling cascade to activate production of proinflammatory cytokines

1.2. Shows the activation of macrophages to produce cytokines TNF $\alpha$, IL1 and IL12.

1.3. Timeline of the study showing E+ and E- rats maintained in different thermal environments.

1.4. Average daily feed intake of Control, Exp. 1 and Exp. 2 groups is shown.

1.5. Average body weight gain of Control, Exp. 1 and Exp. 2 groups is shown.

1.6. Average hourly core body temperature of rats in the Control, Exp. 1 and Exp. 2 groups over the entire study period.

1.7. Average daily core body temperatures of rats in the Control, Exp. 1 and Exp. 2 groups.

1.8. Hourly core body temperature averaged by group and averaged for days 11 to 14 for Control, Exp. 1, and Exp. 2 groups.

1.9. Hourly core body temperature averaged by group and averaged for days 16 to 21 .

1.10. Hourly core body temperature averaged by group and averaged for days 30 and 31

1.11. Hourly core body temperature averaged by group and averaged for days 33 to 36 for Control, Exp. 1, and Exp. 2 groups.

2. Timeline of short term study. Time line of study shows E+, E- and PF $\mathrm{TO} \mathrm{E}+$ rats maintained in pretreatment, diet treatment and thermal treatment

2.1. Average daily feed intake of E-, E+, and PF TO E+ rats during HS and TN periods 
2.2. Change in average daily feed intake of E-, E+, and PF TO E+ during pre treatment period.

2.3. Change in average daily feed intake of E-, E+, and PF TO E+ during thermal treatment period.

2.4. Change in average daily body weight gain of E-, E+ and PF TO E+ rats maintained at $\mathrm{HS}$ and $\mathrm{TN}$ conditions during the entire study......

2.5. Change in average daily body weight of E-, E+, and PF TO E+ groups during diet treatment at $\mathrm{TN}$

2.6. Change in average daily body weight of E-, E+, and PF TO E+ during thermal and diet treatment period

2.7. Change in average core body temperature of E-, E+, and PF TO E+ rats maintained under HS and TN conditions.

2.8. Change in average core temperature of E-, E+, and PF TO E+ during the diet treatment period....

2.9. Change in average core body temperature of E-, E+ and PF TO E+ during the thermal and diet treatment period...

2.10. Circadian changes in average core body temperature of E-, E+, and PF TO E + during the diet treatment period

2.11. Circadian changes in average core body temperature of E-, E+, and PF TO E+ during the thermal and diet treatment period....

2.12. Mean gross motor activity of E-, E+, and PF TOE+ groups during the thermal and diet treatment period...

2.13. Circadian changes in gross motor activity of E-, E+, and PF TOE+ groups during the diet treatment period...

2.14. Circadian changes in gross motor activity of E-, E+ and PF TOE+ groups during the thermal treatment period.

2.15. Horizontal bars represent E-, E+, and PF TO E+ percentages of natural killer cells under HS and TN conditions

2.16. Horizontal bars represent E-, E+, and PF TO E+ percentages of Blymphocytes cells under $\mathrm{HS}$ and $\mathrm{TN}$ conditions

2.17 Horizontal bars represent E-, E+, and PF TO E+ percentages of Tlymphocytes for rats under HS and TN conditions.

2.18. Change in average core body temperature of E-, E+ and PF TO E+ in response to LPS challenge at TN... 
2.19. Change in average core body temperature of E-, E+ and PF TO E+ in response to LPS challenge under HS conditions.

2.20. Horizontal bars represent levels of circulating IL1 $\beta$ in E-, E+, and PF TO E+ 2 hours after LPS challenge at TN and under HS conditions...

2.21 Horizontal bars represent levels of circulating TNF $\alpha$ in E-, E+ and PF TO E+ 2 hours after LPS challenge at TN and under HS conditions...

3. Timeline of long term study. Time line of study shows E+, E- and PF $\mathrm{TO} \mathrm{E}+$ rats maintained in pretreatment, diet treatment and thermal treatment.

3.1. Average daily feed intake of E-, E+, and PF TO E+ rats under HS and TN conditions

3.2. Change in average daily feed intake of E-, E+, and PF TO E+ during diet treatment period.

3.3. Change in average daily feed intake of E-, E+, and PF TO E+ during heat stress and diet treatment period.

3.4. Average daily body weight gain of E-, E+, and PF TO E+ rats maintained under HS and TN conditions...

3.5. Change in average daily body weight intake of E-, E+ and PF TO E+ during diet treatment period.

3.6. Change in average daily body weight of E-, E+, and PF TO E+ during HS and diet treatment period.

3.7. Average daily core body temperature of E-, E+, and PF TO E+ rats maintained under HS and TN conditions during entire study period...

3.8. Change in average daily core body temperature of E-, E+, and PF TO $\mathrm{E}+$ during diet treatment.

3.9. Change in core body temperature of E-, E+, and PF TO E+ during first three days of HS and diet treatment period.

3.10. Change in core body temperature of E-, E+, and PF TO E+ of rats maintained under HS and TN conditions.

3.11. Circadian changes in core body temperature of E-, E+, and PF TO E+ rats during diet treatment period.

3.12. Circadian changes in core body temperature of E-, E+, and PF TO E+ rats maintained under $\mathrm{HS}$ and $\mathrm{TN}$ conditions. 
3.13. Average daily activity of E-, E+, and PF TO E+ rats maintained under HS and TN conditions

3.14. Circadian changes in average daily activity of E-, E+, and PF TO E+ rats during diet treatment period

3.15. Circadian changes in average daily activity of E-, E+, and PF TO E+ rats maintained under HS and TN conditions.

3.16. Horizontal bars represent E-, E+, and PF TO E+ percentages of natural killer cells maintained under TN and HS conditions

3.17. Horizontal bars represent E-, E+, and PF TO E+ percentages of $\mathrm{T}$-lymphocytes maintained at $\mathrm{TN}$ and $\mathrm{HS}$ conditions.

3.18. Horizontal bars represent E-, E+, and PF TO E+ percentages of CD4+ cells maintained under TN and HS conditions

3.19. Horizontal bars represent E-, E+, and PF TO E+ percentages of CD8+ cells maintained under TN and HS conditions

3.20. Average core body temperature of E-, E+, and PF TO E+ rats in response to LPS at thermoneutrality...

3.21. Average core body temperature of E-, E+, and PF TO E+ rats in response to LPS under HS conditions

3.22. Horizontal bars represent levels of circulating IL1 $\beta$ in E-, E+, and PF TO E+ 2 hours after LPS challenge at TN and under HS conditions...

3.23. Horizontal bars represent levels of circulating TNF $\alpha$ in E-, E+ and PF TO E+ 2 hours after LPS challenge at TN and under HS conditions... 


\section{LIST OF TABLES}

Tables

1. Important cells and physiologic functions of innate and adaptive

immune system. Abbas

1.1. The table shows different $\mathrm{T}$ cell surface receptors and depicts Th1, Th2 and Th17 pathways of CD4+ Cells.

1.2. The arrows indicate significant increase or decrease when compared to the E-TN group in the short term study

1.3. The arrows indicate significant increase or decrease when compared to the E-TN group in the long term study 


\section{INTRODUCTION}

Fescue toxicosis is caused by the fungus Neotyphodium coenophialum that is sometimes present in tall fescue grass (Lolium arundinaceum) and makes the fescue heat and drought tolerant (Malinowski et al., 2005). Tall fescue is widely used in the midwestern United States as pasture grass, and more than 50\% of the tall fescue in the United States is infected by this fungus (Jones et al., 2004). Every year more than 8.5 million cattle and 700,000 horses graze on tall fescue pasture (Stuedmann and Seman, 2005). A conservative estimate by Hoveland (1993) is that there is a loss of over 600 million dollars annually to the beef industry alone due to fescue toxicosis. Similarly, a more recent estimate is that there is an annual loss of over 1 billion dollars to the livestock industry (Panaccione et al., 2001).

Various ergopeptines alkaloids are produced by the fescue plant and fungus symbiotic relationship, which include ergonine, ergovaline, ergogotamine and lysergic acids (Porter, 1995). Of these, ergovaline is the most potent vasoconstrictor

(Lyons et al., 1986; Aiken et al., 2007). These toxins bind to adrenergic, serotonergic and dopaminergic receptors which result in reduced feed intake (FI), body weight (BW) gain, and altered thermoregulatory system (Cross et al., 1995; Oliver, 2005). The reproductive performance is affected in both male and female animals (Collier et al., 2006). Animals affected by these toxins include cattle (Cross et al., 1995; Oliver, 2005), horses (Cross et al, 1995), rabbits (Filipov et al., 1998), sheep (Gadberry et al., 2003), rats (Spiers et al., 2005) and mice (Varney et al., 1991).

Similarly, heat stress decreases production in cattle and other farm animals (West, 2003). Heat stress reduced FI and body weight gain in rats with fescue toxicosis (Settivari et al., 2006). In the United States, heat stress causes an annual 
economic loss to the animal industries of $\$ 1.69$ to $\$ 2.36$ billion dollars (St-Pierre et al., 2003). The dairy industry is affected the most (\$897 to $\$ 1500$ million dollars), followed by the beef industry (370 million dollars), the swine industry ( $\$ 299$ to $\$ 316$ million dollars) and the poultry industry (\$128 to $\$ 165$ million dollars) (St-Pierre et al., 2003).

Different responses to fescue toxin in thermoneutral and heat stress conditions have been documented using the rat model. Interestingly, upon exposure to fescue toxins, rats decrease their core body temperature at thermoneutrality (Zhang et al., 1994) and increase their core body temperature under heat stress conditions (Osborn et al., 1992; Spiers et al., 2005). This underscores the importance of studying fescue toxicosis in both thermoneutral and heat stress conditions.

Effects of fescue toxicosis on the immune system have been variable. Studies using cattle and mice have shown that fescue toxicosis lowers white blood cell count, mononuclear phagocytic activity and serum prolactin levels (Saker et al., 1998; Filipov et al., 1999a; Dew et al., 1990; Settivari et al., 2006). Similarly, administered ergot alkaloids decreased eosinophil count and plasma $\gamma$-globulin level, and increased clot formation and release of acute phase proteins (Oliver et al., 2000). In a long-term study of mice fed an endophyte infected diet at thermoneutrality, Dew et al. (1990) reported an increase in $\mathrm{T}$ suppressor cells in the splenocytes of mice.

Few studies have attempted to differentiate the effects of immune system under thermoneutral (TN) and heat stress (HS) conditions. Likewise, studies to identify the effects of fescue toxicosis, heat stress, caloric restriction and their interaction have not been performed. In our study, we hypothesized that the combined effect of heat stress and fescue toxicosis could affect the immune system more than either acting alone. 
We also hypothesized that long-term heat stress and fescue toxicosis will allow the animal to adapt to these stressors. 


\section{LITERATURE REVIEW}

\section{FESCUE TOXICOSIS}

\subsection{Introduction}

Tall fescue is widely used as pasture grass in the United States, and is the most important cool season grass for farm animals (Sleper and Buckner, 1995). It is considerably resistant to insect attacks and thrives in marginal, acidic and poorly drained soils, in areas of low fertility, and in zones stressed by overgrazing and drought (West, 2003). These advantages are related to its symbiotic relationship with the fungus Neotyphodium coenophialum (Malinowski et al., 2005). However, this relationship is the primary cause of toxicosis in animals and warrants a detailed analysis to delineate the various changes in the system.

\subsection{Toxins in tall fescue grass}

Research has shown that fescue plant produces alkaloids that include pyrrolizidines (lolines), ergot alkaloids (ergovaline, clavines, lysergic acid, ergosine, and ergonine) and pyrrolopyrazine (peramines) (Porter, 1995). Ergovaline accounts for more than $85 \%$ of the ergot alkaloids present in the plant (McCollough et al., 1994). Ergovaline is the most potent vasoconstrictor of all the ergot alkaloids present in the plant (Aiken et al., 2007) and has a dose dependent vasoconstrictor action on the caudal artery of beef cattle. Peramines and lolines are also isolated from the plant, but they do not have any significant effects on animals (Porter, 1995). 
Ergoline alkaloids (lysergic acid) may be responsible for clinical signs associated with fescue toxicosis (Hill et al., 2001), with their absorption being greater compared to other toxins in ruminants. Hill (2005) proposed that lysergic acid was responsible for most of the clinical signs associated with fescue toxicosis. In contrast, Klotz et al. (2006) showed that lysergic acid is a weak vasoconstrictor and does not cause vasoconstriction at lower doses. Similarly, in vivo and in vitro studies have shown that ergovaline is a potent vasoconstrictor and causes hyperthermia in rats (Strickland et al., 1993; Spiers et al., 1995). Although ergovaline is accepted as the primary contributor to symptoms associated with fescue toxicosis, there is the possibility that other toxins may contribute to some of the signs of fescue toxicosis (Gadberry et al., 2003).

Erogovaline concentrations in plants can vary from 2 to 6 microgram/gram (Porter, 1995), with effects of soil nitrogen and season on these levels. Therefore, it is important to know the level of ergovaline concentration in forages by animals (Porter, 1995). Ergovaline's toxic concentration is dependent on species of animal studied. Horses tolerate a dietary level of $500 \mathrm{ppb}$, cattle tolerate a maximum of 500 to 700 ppb, and sheep tolerate a maximum of 1000 to 1500 (Cross et al., 1995).

\subsection{Fescue toxicosis and thermoregulation.}

Fescue toxicosis affects the thermoregulatory system in various ways. One way is to bind adrenergic and serotonergic receptors to cause vasoconstriction (Oliver, 2005). The result is that vasodilation is reduced along with the release of heat from the body. Moreover, the binding to adrenergic receptors causes vasoconstriction of bronchioles, which results in an additional strain on heat loss through respiration (Osborn et al., 1992). The other mechanism is the inhibition of the release of nitric 
oxide which aids in vasodilation (Al-Tamimi et al., 2007). Because of these effects on the thermoregulatory system, there is a greater increase in the core body temperature of animals subjected to both heat stress and endophyte (Spiers et al., 2005; Settivari et al., 2006). Another interesting feature observed with fescue toxicosis is hypothermia in thermoneutral or cold environments (Neal and Schmidt, 1985; Osborn et al., 1992; Spiers et al., 1995). Hypothermia associated with fescue toxicosis in the rat at thermoneutrality is attributed to a decrease in metabolic heat production rate and increased heat loss from the rat's tail (Spiers et al., 1995). This underscores the importance of studying animals in both conditions, because the effects could be markedly different.

\subsection{Fescue toxicosis and feed intake}

Fescue toxicosis causes a significant reduction in FI of most animals (Hemken et al., 1981). This decrease in FI is documented in cattle, sheep (Schmidt et al., 1982; Osborn et al., 1992) and rats (Spiers et al., 2005). The effect of fescue toxicosis on FI is greater during heat stress (Hemken et al., 1981; Spiers et al., 2005; Settivari et al., 2006). This reduction is related to the toxins present in the feed, and is not due to the consistency of seed in the diet or palatability of feed (Spiers et al., 2005). Ergot alkaloid impact on the autonomic nervous system could be one avenue for alteration of FI. Several neurotransmitters affect FI and gut motility. Ergot alkaloids can bind $\alpha-2$ adrenergic, dopaminergic, serotonergic, and hydroxytryptamine (5-HT) receptors (Cross et al., 1995; Oliver, 2005). Serotonin plays an important role in satiety following food consumption. There is a dose-dependent reduction in FI of rats in response to serotonin injection (Pollock and Rowland, 1981). In the rat model, injection of $\alpha$-adrenergic receptor agonist into the paraventricular nucleus (Wellman 
et al., 1993) and intraperitoneal injection of dopamine causes a reduction in FI

(Bednar et al., 1991). Likewise, prazosin (an $\alpha$-adrenergic antagonist) administered to rats intraperitoneaaly produced a significant increase in FI under heat stress conditions (Larson, 1993). In steers intramuscular injection metoclopromide (dopamine antagonist) increased FI, average daily weight gain, serum prolactin and cholesterol levels (Lipham et al., 1989; Rhodes et al., 1991). These reports suggest that the effect of fescue toxicosis on FI is receptor mediated. Although these measures improved FI and prolactin levels, their ability to alleviate thermal strain was limited

\subsection{Effects of fescue toxicosis on body weight and growth rate}

Fescue toxicosis causes a reduction in FI which leads to decreased body weight gain. These effects are observed both in thermoneutral and heat stress conditions. A marked (70-78\%) reduction in FI and average daily weight gain was observed in rats that received an endophyte-infected seed diet during heat stress compared to animals at thermoneutrality (Spiers et al., 2005). Other researchers have also documented significant reductions in FI and body weight gain (22 to 79\%) of cattle under different ambient temperatures (Schmidt et al., 1982). Similar to other features, the combined effects of heat stress and endophyte-infected fescue causes the largest reduction in body weight gain in rats and livestock (Gadberry et al., 2003; Spiers et al., 2005; Settivari et al., 2006). Hypoprolactemia could be a possible contributing factor to the reduced FI and body weight gain (Cross et al., 1995). Attempts to separate the effects of fescue toxicosis and caloric restriction have shown that fescue toxicosis by itself can affect growth rate, nitrogen digestibility, and reproductive performance of farm animals (Varney et al., 1991) 


\subsection{Effects of fescue toxicosis on prolactin}

Consumption of endophyte-infected fescue causes a significant reduction in serum prolactin level in cattle, sheep, and rats (Mizinga et al., 1993; Thompson et al., 2001; Gadberry et al., 2003). Binding of ergopeptine alkaloids to dopaminergic receptors (Thompson and Stuedemann, 1993; Oliver, 2005) leads to a decrease in serum prolactin level. Decreased prolactin level could lead to reduced milk production, a dull and shaggy hair coat, reproductive failure, and immunosuppresion (Reber, 1993; Regisford and Katz, 1993; Prasad et al., 1989; Aiken et al., 2006). Serum prolactin level is a very sensitive indicator of fescue toxicosis. Like other hormones, prolactin is connected to various systems in the body and a major reduction could impact normal functioning of these systems.

\subsection{Fescue toxicosis and immune system}

Fescue toxicosis impacts the immune system, and various research teams have attempted to develop procedures to reduce this effect. Studies of cattle and rats show that ergot alkaloids lower white blood cell count, eosinophil count, $\gamma$-globulin level mononuclear phagocytic activity and lymphocyte to neutrophil ratio (Saker et al., 1998; Filipov et al., 1999a; Dew et al., 1990). They also increase clot formation and release of acute-phase proteins in the cattle model (Oliver et al., 2000). Genomic studies have shown reduction in genes corresponding to the T-lymphocyte lineage (Settivari et al., 2006), consistent with previous studies which have reported an increase in T-suppressor cell percentage in rats fed ergot alkaloids at thermoneutrality for 4 weeks (Dew et al., 1990). 
Long-term pasture studies of cattle have suggested that a deficiency of copper in endophyte-infected fescue $(\mathrm{E}+)$ pastures reduces major histocompatibility complex II (MHC) expression and prolactin level in serum. Supplementing of copper in the diet of cattle in endophyte infected fescue pasture, increases MHC II expression (Saker et al., 1998). Similarly, steers grazing on endophyte-infected tall fescue have a lower monocytic phagocyte activity compared to steers on endophyte free fescue (E-) pastures (Saker et al., 2001). Supplementing seaweed Ascophyllum nodosum, with the regular diet increased MHC II expression and phagocytic activity in steers (Saker et al., 2001). Other efforts to improve immune function have used a vaccine for fescue toxicosis and were successful under laboratory conditions (Filipov et al., 1998).

Steers fed an E+ diet for 8 months were injected lipopolysaccharide (LPS) intravenously and showed a higher pro-inflammatory response compared to controls. This was evident from the increased production of cortisol and tumor necrosis factor$\alpha$ (Filipov et al., 1999a). Similar results were obtained using mice which showed an increased inflammatory response in response to an ergotamine containing diet (Filipov et al., 1999b). This suggests that fescue toxicosis in thermoneutrality may not affect proinflammatory cytokine production. Similarly, cattle fed an E+ diet also had normal or increased humoral response when they were challenged with concanavalin and sheep red blood cells (Rice et al., 1997). In a separate study, Dew et al. (1990) documented a 42\% increase in T suppressor cells of mice fed an E+ diet for 43 days. Contrary to all other studies, this study showed a decrease in humoral immune response to concanavalin and LPS challenges. The effect of endophyteinfected tall fescue on immune system function varies with environmental 
temperature, dose of toxin, period of exposure, and health status of animals under observation.

\section{HEAT STRESS}

\subsection{Thermoregulation}

Homeotherms have the ability to regulate their body temperature, in spite of variations in ambient temperature (Baker, 1989). Metabolism and heat gained from the environment are two important sources of heat accumulation in the body. This gained heat must be regulated and dissipated to maintain a normal core body temperature (Knochel and Reed, 1994). Thermoreceptors in the periphery and central nervous system send thermal information to the hypothalamus in the brain (Baker, 1989). The rostral cooling center in the hypothalamus stimulates the medial satiety center, which in turn inhibits the lateral appetite center causing decreased FI and milk production (Albright and Alliston, 1972).

\subsection{Heat loss mechanisms}

Heat loss by conduction, convection, radiation, and evaporation of water are different mechanisms of temperature regulation. Radiation and convection avenues contribute to heat dissipation at low temperatures. Non-evaporative heat loss is reduced at higher temperatures, making animals dependent on convective heat transfer to the skin with peripheral vasodilation and both cutaneous and respiratory evaporative losses to lose heat (Berman et al., 1985). A greater shift to the evaporative avenue occurs as ambient temperature rises closer to skin temperature (Silanikove, 2000). Convection can be broadly classified into natural and forced 
convections. Moving animals or obejectsthrough water or air results in heat transfer to these media via forced convection. Medium density and the thermal gradient between the animal and the surrounding medium help in heat transfer from animal to surroundings by convection (Bligh and Johnson, 1973; Mercer, 2001). There is actually an inverse relationship between convective heat loss and environmental temperature. As air temperature increases above skin temperature, animals begin to gain heat from surroundings (Maia et al., 2005). Velocity of the surrounding media also affects the rate of convective heat transfer (Silanikove, 2000).

Similarly, animals can also lose body heat by radiant exchange. All objects with a temperature above absolute zero release infrared electromagnetic energy (Mercer, 2001). Radiant heat transfer occurs from a higher to lower temperature (Curtis, 1983), therefore animals and their surroundings can gain heat from the sun during the day and lose it in the night. Similarly, skin color can influence radiant heat exchange, and this has been documented using cattle with different skin colors (Hutchinson and Brown, 1969). Animals with lighter coat reflect radiation, while animals with darker coat absorb more radiation in the visible portion of the electromagnetic spectrum.

Conduction is heat loss from animal to any stationary object that is in contact with the animal. For instance, contact with any media surrounding the animal could help in conductive heat loss (Silanikove, 2000). Heat loss by conduction is influenced by the thermal gradient between the exchanging surface, conductivity of the media, and the area of contact with the animal (Schmidt-Nielson et al., 1952).

Evaporative heat loss occurs from cutaneous and respiratory systems. Respiratory and cutaneous diffusion causes passive loss of heat (Yousef, 1985) in cattle. An increase in blood temperature increases sweating rate (Buono and Sjoholm, 1988) which increases heat loss by evaporation. Previous data suggests that 
evaporation of 1.7 milli litres of sweat results in the loss of $1 \mathrm{kcal}$ of heat (Buono and Sjoholm, 1988). Likewise, human studies have shown that up to $600 \mathrm{Kcal}$ heat can be lost in a single day by sweating in a dry environment (Buono and Sjoholm, 1988) making it a very efficient heat loss mechanism in extreme heat, if humidity is conducive for evaporation.

\subsection{Heat stress and immune system}

In response to heat stress, endothelial and epithelial cells, and leukocytes undergo a complex reaction to protect the body from injury (Gabay and Kushner, 1999). Various chemical messengers known as cytokines are released from macrophages in response to heat stress. Proinflammatory cytokines like tumor necrosis factor alpha $(\mathrm{TNF} \alpha)$ and interleukin $1 \beta$ (IL1 $\beta)$ are released during heat stress in animals (Bouchama et al., 1991; Lin et al., 1997). Lin et al. (1997) showed that these cytokines can also increase intracranial pressure and cause neuronal damage. These damages are not reversed even if body temperature is reduced to its normal levels. The proinflammatory cytokine response to heat stress is comparable to that of endotoxemia (Abbas et al., 2007). However, few studies have determined the individual and combined effects on the proinflammatory cytokines during heat stress.

\subsection{Heat stress and effects on production parameters}

Heat stress is a major concern of producers in dairy and beef industry. It affects FI, daily bodyweight gain, and reproduction in both sexes (Collier et al., 2006). Heat stress has direct and indirect effects on the animals. Animals consume less during heat stress and the effects of the resulting caloric restriction can be seen in the shifts in both hormonal and acid-base profile of the cattle (El-Noughty et al., 1990; Smith et 
al., 1997; West, 2003). The reduction in caloric intake with heat exposure is greater than the decrease in caloric utilization associated with heat strain (Pereira et al., 2007). This has been documented in field studies using cattle. Like cattle, rats are also susceptible to heat stress as evidenced by a significant reduction in FI and daily bodyweight gain (Johnson and Stack., 1989; Spiers et al., 2005; Settivari et al., 2006).

\section{CALORIC RESTRICTION}

\subsection{Caloric restriction and immune system}

Heat stress and fescue toxicosis reduces FI in animals. Although the average daily gain is reduced, there are a few useful effects of caloric restriction. Caloric restriction of 25 to $50 \%$ for a prolonged period of time, slows down the aging process in rats (Masoro, 2000; Masoro and Austad.,1996; Wanagat et al., 1999). Long-term caloric restriction increases the production of T-lymphocytes in aged rats (Tian et al., 1995). These effects are mediated by a reduction in oxygen-free radicals in the system. In growing male rats, caloric restriction increases the $\mathrm{CD} 4+/ \mathrm{CD} 8+$ ratio and reduces B cell number (Esquifinoa et al., 2004). Likewise, a $60 \%$ caloric restriction decreases the risk of colitis and increases NK cell activity in mice (Shibolet et al., 2002). A caloric restriction of $40 \%$ can reduce the pro-inflammatory response in mice (Muthukumar et al., 2000), but these effects were documented after four to seven months of caloric restriction.

\subsection{Caloric restriction and core body temperature}

Caloric restriction affects core body temperature in rats. It reduces thyroid and growth hormone levels, metabolic rate, and body mass (Kaplan and Utiger 1978; 
Severinsen and Munch, 2001). Severinsen and Munch, (2001) found that restriction of one fourth the caloric value of the control diet reduced core body temperature by $0.04^{\circ} \mathrm{C}$ for every 24 hours, compared to starved rats that reduced core body temperature by $0.1^{\circ} \mathrm{C}$ every 24 hours. Another study of rats (Duffy et al., 1989) showed that resting metabolic rate was lower in caloric restricted and starved rats compared to rats that received feed for ad libitum access. However, the difference between maximum and minimum core temperature was more in rats that received caloric restrictions compared to those that did not (Duffy et al., 1989). This leads to the suggestion that metabolic rate and the energy released as a result of metabolism impact core body temperature (Munch, 1995). Likewise, rats maintained in E+ diet at thermoneutrality exhibit reduced FI and core body temperature (Spiers et al., 2005; Settivari et al., 2006). Genomic analysis (Settivari et al., 2006) has also supported the idea of down regulation of genes associated with energy metabolism.

\section{INTERACTION BETWEEN STRESSORS}

\subsection{Effects of heat stress and fescue toxicosis on feed intake}

It is a well known that animals reduce their FI in response to fescue toxicosis, heat stress, and the combination of both (Aldrich et al., 1993; Larson et al., 1999). However, for a long time, the effects of stressors acting alone were unknown. Later, it was documented that rats decrease their FI in both thermoneutral and heat stress conditions when fed with an E+ diet (Spiers et al., 1995, 2005). Rats fed E+ diet in thermoneutrality showed an adaptation to FI and increased their FI after three to four days of exposure to ergot alkaloids (Roberts et al., 2002; Spiers et al., 2005). Similarly, rats which received E- diet in heat also showed an increase in feed intake 
after three days in heat stress (Spiers et al., 2005). However, the combination of heat stress and $\mathrm{E}+$ produce a greater reduction in FI compared to either acting alone (Spiers et al., 2005; Settivari et al., 2006).

\subsection{Effects of heat stress and fescue toxicosis on core body temperature}

Peripheral vasoconstriction occurs as a result of fescue toxicosis, and diminishes heat loss to result in an increase in core body temperature. A reduction in nitric oxide caused by fescue toxicosis prevents vasodilation in cattle and supplementing nitric oxide helps cattle dissipate heat.(Oliver et al., 2000). This study reported a reduction in plasma arginine (a precursor of nitric oxide), nitrite and nitrate levels reduced nitric oxide in peripheral blood and hamper vasodilation in cattle. Previous studies done using the rat model have shown that supplementing nitric oxide increases vasodilation and alleviates the effects of HS caused by the endophyte infected diet (Al-Tamimi et al., 2007). The bovine model also showed that supplementing nitric oxide reverses the effect of endophyte in HS conditions (Al-Tamimi et al., 2007). This shows that supplemeting nitric oxide aides in vasodilation and protects the animal from harmful effects associated with increased core body temperature.

Heat stress, fescue toxicosis, and their combination cause an increase in core body temperature, Rats that received E- diet and heat stress $\left(31^{\circ} \mathrm{C}\right)$ were able to adapt and returned to the preheat core body temperatures (Settivari et al., 2008). The same study showed that E+ HS rats continue to increase their body temperature and a lack of adaptation was seen in the short term study. This might be due acute stress and rats might need more time to significantly reduce their core body temperature. However, Settivari et al. (2008) reported an adaptation of rats in the long term (3 weeks) HS study $\left(31^{\circ} \mathrm{C}\right)$ and significant reductions in core body temperatures after 3 days in HS. 


\subsection{Effects of stressors on gross motor activity}

Animal activity can be an indication of health status. Sick rats have lowered activity compared to healthy rats due to both the infections and psychological disorders associated with illness (Meerlo et al., 1996; Luker et al., 2000). Similarly, caloric restriction of $75 \%$ caused a greater reduction in activity compared to rats $25 \%$ caloric restriction in TN conditions reduces (Severinsen and Munch, 2001). Spiers et al. (2005) reported a trend towards reduced activity in TN conditions compared to pretreatment levels.

The circadian activity follows the same pattern as that of core body temperature rhythm in rats (Scales and Kluger, 1987). The circadian pattern is dependent on light in the natural world. The day light cycles helped rats maintain a circadian rhythm in experimental chambers through its effects on suprachiasmatic nucleus in the brain (Saper and Breder, 1994). Gordon, (1993) reported a increase in activity at 2100 after lights were turned off and a decrease from 0600 to 0700 when lights were turned on. These effects were seen in rats fed endophyte infected diet and maintained at HS conditions. This suggests that endophyte and HS does not alter the circadian pattern completely, rather makes the rats follow the same circadian pattern at a higher temperature.

\section{IMMUNE SYSTEM}

\subsection{Immune system overview}

The immune system protects the body against invading pathogens and microorganisms. This system can be broadly classified in to innate immunity and adaptive immunity (Flajnik and Pasquier, 2004). In the innate immune system, the 
host's protective mechanisms are non-specific (Abbas et al., 2007). These include physical barriers like skin, mucous membranes, phagocytes, cytokines and natural killer cells (Flajnik and Pasquier, 2004). Phagocytes of innate immune system are neutrophils, monocytes, macrophages, dentritic cells and natural killer cells (Figure a). Macrophages are a major source of cytokines and nitric oxide. Cytokines are polypeptides released in response to microbial pathogens and they assist in immune regulation and inflammation. Pro-inflammatory cytokines TNF $\alpha, 1 \mathrm{~L} 1$, interferon gamma (IFN $\gamma)$ and IL12 are important cytokines of innate immune response (Table. 1). Neutrophils and macrophages along with cytokines, initiate a complex inflammatory cascade, which is an essential feature of innate immune system. 


\begin{tabular}{|c|c|c|}
\hline Features & Innate immunity & Adaptive immunity \\
\hline Examples & TNF, $\|L-1\| L-12,, I F N-\gamma^{*}$ & $\|L-2\| L-4,, \| L-5, \mid \mathbb{F N}-\gamma^{*}$ \\
\hline Major cell source & Macrophages, NK cells & Tlymphocytes \\
\hline $\begin{array}{l}\text { Principal physiologic } \\
\text { functions }\end{array}$ & $\begin{array}{l}\text { Mediators of inflammation } \\
\text { (local and systemic) }\end{array}$ & $\begin{array}{l}\text { Regulation of lymphocyte growth and } \\
\text { differentiation; activation of effector cells } \\
\text { (macrophages, eosinophils, mast cells) }\end{array}$ \\
\hline Stimuli & $\begin{array}{l}\text { LPS (endotoxin), bacterial peptidoglycans, } \\
\text { viral RNA, T cell-derived cytokines (IFN-y) }\end{array}$ & Protein antigens \\
\hline Amounts produced & May be high; detectable in serum & Generally low; usually undetectable in serum \\
\hline $\begin{array}{l}\text { Local or systemic } \\
\text { effects }\end{array}$ & Both & Usually local only \\
\hline Roles in disease & Systemic diseases (e.g., septic shock) & $\begin{array}{l}\text { Local tissue injury (e.g., granulomatous } \\
\text { inflammation) }\end{array}$ \\
\hline Inhibitors & Corticosteroids & Cyclosporine, FK-506 \\
\hline
\end{tabular}

${ }^{\star} \mid \mathrm{FN}$ - p plays important roles in innate and adaptive immunity.

Abbreviations: IFN, intereferon; IL, interleukin; LPS, lipopolysaccharide; NK, natural killer; TNF, tumor necrosis factor

Table 1. Important cells and physiologic functions of innate and adaptive immune system. Abbas et al., 2007. 
Inflammation is a response by the host and sets in as soon as the innate immune system receives signals of impending danger. Macrophages, neutrophils and mast cells release inflammatory mediators such as cytokines, to initiate a complex inflammatory cascade (Figure 1; Abbas et al., 2007). Bradykinins, leukotrienes, nitric oxide and histamine aid in vasodilation and augment movement of inflammatory cells to the tissues or places were the pathogen is present. Pro-inflammatory cytokines, IL1 and TNF $\alpha$, cause a wide range of responses which include attraction of neutrophils, leukocyte adherence, migration to site of injury, activation of fibroblasts and fever (Figure 1; Abbas et al., 2007). Although various other cytokines are involved in the innate immune system, IL1, TNF $\alpha$ and 1L6 play a central role in innate immune response and thus have been studied extensively (Abbas et al., 2007). 


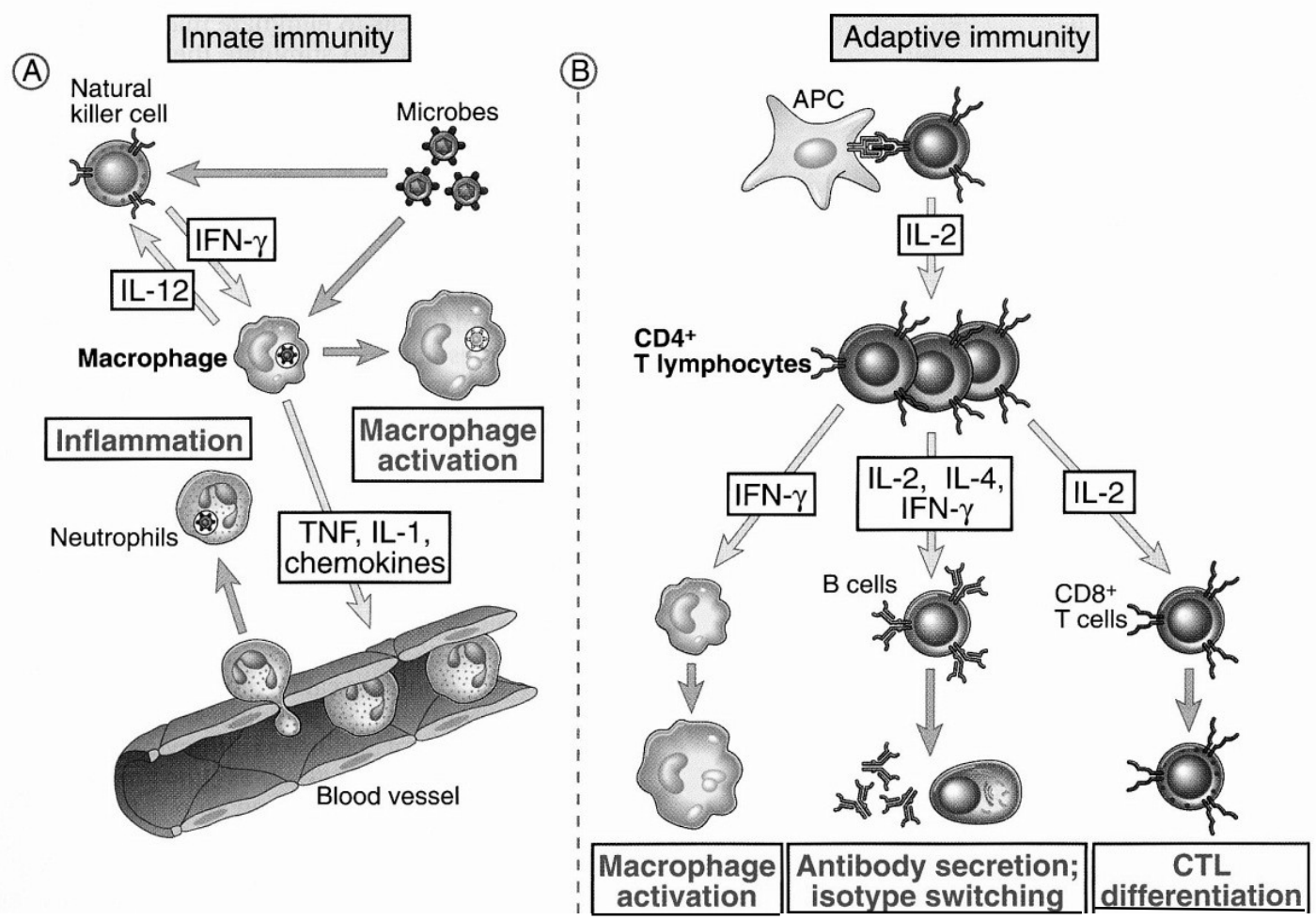

Figure 1. Innate immune response and immune cells associated with the process of inflammation. The T-lymphocyte (CD4+ and $\mathrm{CD} 8+)$ and B-lymphocytes role in adaptive immunity are also portrayed. Abbas et al., 2007 . 
In contrast to innate immunity, the adaptive immune system has specificity, diversity and immunologic memory (Flajnik and Pasquier, 2004). Specificity of adaptive immune system initiates specific response against distinct pathogens. Cells of adaptive immunity such as lymphocytes have specific membrane receptors which can detect subtle differences in structure of different antigens (Abbas et al., 2007). Diversity helps the adaptive immune system to act against different types of antigens. Immunologic memory within the system means that it mounts a more rapid and larger response on subsequent exposure to the same pathogen (Flajnik and Pasquier, 2004). For example memory $\mathrm{B}$ cells produce more antibodies and $\mathrm{T}$ cells react more rapidly and vigorously when exposed to the pathogen compared to naïve cells. Adaptive immunity is broadly classified in to humoral immune system and cell-mediated immune system (Abbas et al., 2007). Humoral immunity is characterized by formation of antibodies from B-lymphocytes. Antibodies attach to foreign antigens and aid in phagocytosis and destruction of these antigens. Cell-mediated immunity is mediated by T-lymphocytes (Figure 1; Abbas et al., 2007). T-lymphocytes are characterized by the expression of certain markers on their surface. The major categories of T-lymphocyte subsets are cluster of differentiation $4+, 8+$ and $3+$ (CD4+, CD8+ and CD3+). The CD4+ (helper-T cells) and CD8+ (cytotoxic-T cells) cells are activated based on the type and location of microbes or antigens in the body (Figure 1; Abbas et al., 2007). The primary functional difference between CD4+ and CD8+ T-cells is the ability of CD8+ cells to act against intracellular pathogens and CD4+ cells ability to act against extracellular pathogens. The CD4+ CD8+ percentages have been widely used in the diagnosis of immunosuppressive conditions like HIV (Taylor et al., 1989). Many studies have reported that a balance is needed 
between these cells for the proper functioning of the immune system (Ander Ryst et al., 1998). The host responds through cell-mediated or humoral immune system or both depending on the nature and location of the invading pathogen (Abbas et al., 2007). The functions of immune system is very complex and it is very important to achieve a moderate and desired response because over or underactivation of the immune system can cause undesired effects.

\subsection{Lipopolysaccharide admnistration}

\subsubsection{Introduction}

Gram negative bacteria cause a wide variety of clinical diseases in animals and humans (Abbas et al., 2007). Lipopolysaccharide (LPS) is a major component of gram negative bacteria. A natural infection caused by gram negative bacteria can be simulated in a research laboratory using lipopolysaccharide challenge (Blackwell and Christman, 1997; Hawinger, 2001).

\subsubsection{Mechanism of action}

When LPS is injected, it forms a complex (Figure c) and attaches to the TLR4 receptor which activates the nuclear factor kappa $(\mathrm{NF}-\kappa \mathrm{B})$ signalling cascade (Figure 1.1; Miyake et al., 2007). The NF- $\kappa B$ signalling cascade initiates a series of

phosphorylation events to inhibit кappaB. Once, this molecule is phosphorylated it releases Nuclear factor- $\kappa \mathrm{B}(\mathrm{NF}-\kappa \mathrm{B})$ to initiate transcription of various proinflammatory cytokines IL1, TNF $\alpha$ and 1L6 (Figure 1.1; Blackwell and 
Christman, 1997; Hawinger, 2001). The LPS signalling cascade is illustrated in the Figureure given below. 

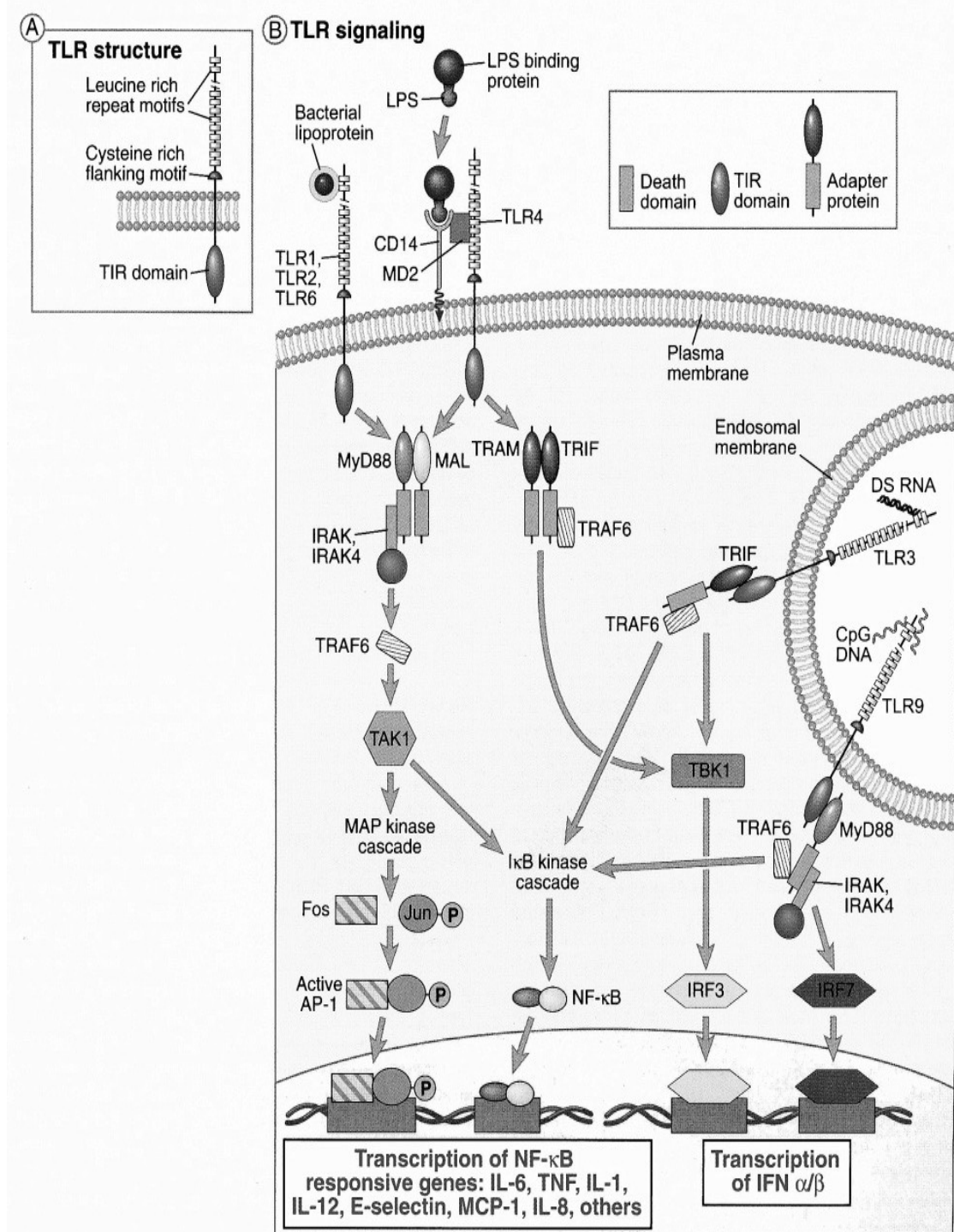

Figure 1.1. Shows association of TRL4 to LPS, followed by a downward signalling cascade to activate production of proinflammatory cytokines. Abbas et al., 2007 


\subsubsection{Response to LPS}

Injection of LPS into an animal stimulates an innate immune response causing a marked increase in several proinflammatory cytokines (IL1, TNF $\alpha$ and IL6), cortisol levels, as well as inducing fever and decreased gross motor activity (Bison et al., 2009). The proinflammatory cytokines TNF $\alpha$, IL1 and IL6 are released in response to LPS and their effects in causing fever are overlapping (Blatteis, 2000; Abbas et al 2007). Proinflammatory cytokines initiate production cyclooxygenase-2, which enhances the production of prostaglandin E2. Elevation of Prostanglandin E2 in the central nervous system, produces fever (Blatteis, 1997). 


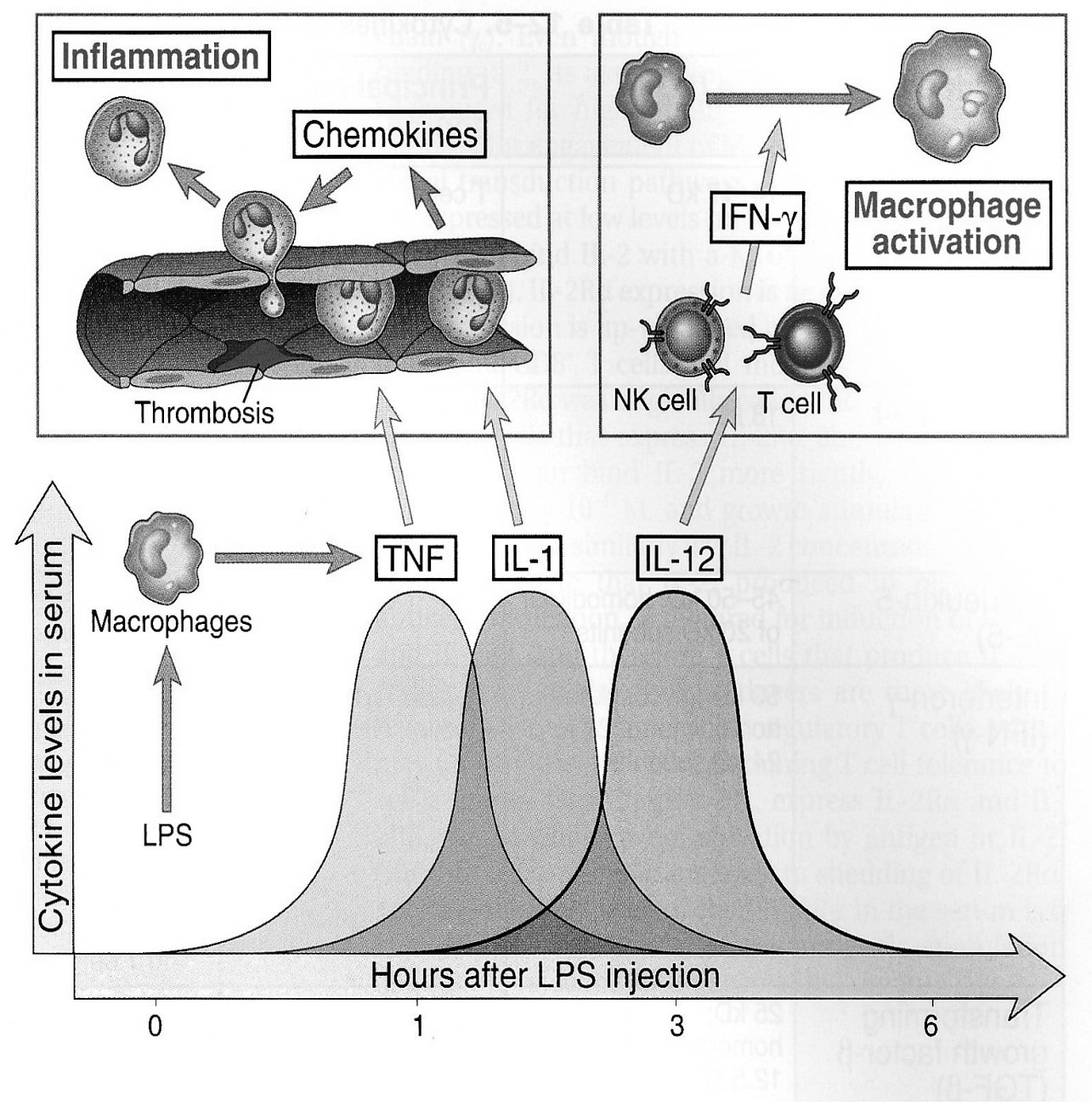

Figure 1.2. Shows the the activation of macrophages to produce cytokines TNF $\alpha$, IL1 and IL12. These cytokines act on the endothelial cells to initiate inflammation. Cytokines release at different time frames is also depicted in the picture. Abbas et al., 2007. 
The inflammatory process in response to LPS is rapid and dynamic. Likewise, TNF $\alpha$ and IL1 levels in response to LPS are different at various time points after LPS challenge. It is widely accepted that a both cytokines are released in greater quantities within 2 hours of LPS challenge (Chu et al., 1997, Ellis et al 2005; Abbas et al., 2007). It is interesting to note that TNF $\alpha$ peaks first, about 1 to 2 hours after LPS challenge and then returns to baseline after 3 to 4 hours of LPS challenge (Figure 1.2; Zuckerman and Butter, 1989; Abbas et al., 2007). The pace at which the levels decline in peripheral blood is dependent on the levels of corticosterone in the blood (Zuckerman and Butter, 1989; Goujon et al., 1997). Similarly, IL1 peaks around 2 hours and then stays above baseline for more than 24 hours (Zuckerman and Butter, 1989; Bison et al., 2009). Therefore, while estimating levels of cytokines in serum samples from peripheral blood, it is important to consider the time at which samples were collected after LPS challenge.

Proinflammatory cytokines are important mediators of sickness behaviour in the mice and rat model (Kent et al., 1992; Bison et al., 2009) and reducing the levels of cytokines by increasing corticosterone is a major adaptive mechanism by the hosts' immune system (Zuckerman and Butter, 1989; Goujon et al., 1997). Reduced activity, anorexia, lethargy, fever, adipisia and reduced social interaction have also been observed in the mice and rat model, when challenged with LPS injection (Weil et al., 2006; Bison et al., 2009). These responses are collectively known as sickness behaviour. This is a part of the hosts' adaptive mechanism to help in recovery from LPS challenge (Weil et al., 2006).

Proinflammatory cytokines stimulate the liver to release acute phase proteins such as fibrinogen, beta microglobin, cystein proteinase inhibitor, serum amyloid A 
and C-reactive protein which play a significant role in the innate immune response (Abbas et al., 2007). These acute phase proteins aid in phagocytosis or recruitment of more inflammatory cells to the site of LPS injection.

\subsubsection{LPS and core body temperature}

Research done in the mice model has show an increased core body temperature in response to LPS challenge (Abbas et al., 2007). However, reports on core body temperature response to LPS in the rat model have not been consistent. A few papers have reported that an increase in body temperature is observed in rats in response to LPS challenge in thermoneutral conditions (Klir et al., 1993; Ellis et al., 2005). These studies reported a significant increase in core body temperature and relate its effect to increased production of proinflammatory cytokines in thermoneutral conditions.

Kluger et al. (1997) and Chu et al. (1997) reported a mild increase in core body temperature after rats were heat stressed before LPS challenge. Many other research groups have stated that rats do not experience an increase their core body temperature. Many groups report that the rat model experiences hypothermia in thermoneutral conditions in response to LPS challenge (Lylte et al., 1973; Ishikawa and Hayaishi, 1982; Kasting and Martin, 1983; Chu et al., 1997; Bison et al., 2009). For example in a recent study doses of $1,5,15,50,125$ and $250 \mu \mathrm{g} / \mathrm{kg}$ BWT were used for IP LPS challenge and rats were monitored over a period of 24 hours following LPS challenge to monitor core body temperature, activity and cytokine response (Bison et al., 2009). This study reported that there was no significant increase in core body temperature in the first two hours of study (Bison et al., 2009). These authors showed that a dose of $250 \mu \mathrm{g} / \mathrm{kg}$ BWT produced hypothermia at 2 hours of LPS challenge. However, all rats used in the study responded with an increased cytokine production as expected. 
This observation suggests that LPS had taken effect and it had minimal effect on core body temperature and activity in rat model.

\subsection{IL 1 (Interleukin 1)}

Interleukin1 was the first cytokine discovered, however scientist later discovered that it is produced after TNF $\alpha$ in response to an infection (Dinarello et al., 1986). Actions of both these cytokines are overlapping as proinflammatory cytokines and share many common features (Dinarello, 1991). There are two separate IL1 $\alpha$ and IL1 $\beta$ receptors (Sims et al., 1994). Injecting IL1 produced fever in animals and blocking IL1 receptor signalling can prevent fever (Chai et al., 1996). IL1 when secreted in low quantities caused local effects, however, in higher concentrations it had systemic effects. When secreted in higher quantities IL1 can directly activate CD4+ memory cells, and CD4+ memory cells initiate production of antibodies from B-lymphocytes (Abbas et al., 2007).

\subsection{TNF $\alpha$ (Tumor necrosis factor alpha)}

The TNF $\alpha$ is an important mediator of innate immune response in response to gram negative bacteria and other systemic infections (Abbas et al., 2007). TNF $\alpha$ is mainly released from macrophages, mast cells and natural killer cell. The TNF $\alpha$ increases vascular permeability attracts leukocytes such as neutrophils and basophils, to the site of infection (Tracey et al., 1986; Dinarello, 1991). This function has both useful and harmful effects on host tissues. On the one hand, it increases permeability and allows phagocytic cells to tissues to enhance phagocytosis. On the other hand, increased vascular permeability can lead to shock, sepsis, disseminated intravascular coagulation, and death (Tracey et al., 1987). Structurally, TNF $\alpha$ and IL1 are different 
and IL1 on its own cannot cause septic shock and apoptosis of cells. These effects are mainly mediated by TNF $\alpha$. Production of TNF $\alpha$ stimulates macrophages to produce more IL1. Effects of TNF $\alpha$ and IL 1 are overlapping and their role in producing fever was indistinguishable (Dinarello et al., 1986).

\subsection{Lymphocytes}

During development of immune cells, they are designated as lymphoid or myeloid progenitors. Lymphoid progenitors result in the formation of T-lymphocytes, B-lymphocytes and natural killer cells (Kondo et al., 1997).

\subsubsection{T-lymphocytes differentiation and maturation}

Mature T-lymphocytes are produced in the thymus. Following migration of lymphocyte precursors to the thymus they can either mature into T-lymphocytes or Blymphocytes (Izon et al., 2002). The activation of T-lymphocytes results in production of CD4+, CD8+ and T-regulatory cells. These cells can act as cytotoxic $(\mathrm{CD} 8+)$ or helper cells (CD4+) depending on the nature of infection (Abbas et al., 2007). Precursors of CD4+ T- lymphocytes are transformed to Th1 (T-helper 1), Th2 (T-helper 2) or recently discovered Th-17 (T-helper 17 cells) (Table 1.1; Harrington, 2005). All three have unique effector functions which were initially related to Th1 and Th2 only (Harrington, 2005). Differentiation of Th0 cells into either Th1, Th2, or Th17 cells is mainly related to local cytokine regulation as described in the Figureure given below. The CD4+cells help in countering extracellular organisms and also activate humoral immunity (Figure. 1.1; Abbas et al., 2007). The CD4+ cells aid in immunological memory and help mount an increased response when the host is exposed to the same pathogen subsequently. The CD8+ cells are mostly involved in 
eliminating tumor cells, intracellular organisms and virus lodged within the cell (Abbas et al., 2007).

The $\mathrm{T}$ cell receptors occur in close association with other $\mathrm{T}$ cell surface molecules. CD4+ and CD8+ are glycoproteins in surface of T cells, which mediate the interaction between the major histocompatibility complex MHC and the T cell receptors. To stimulate T-cell effector functions, an interaction between $\mathrm{MHC}$ and $\mathrm{T}$ cells is needed (Janeway, 1992; Abbas et al 2007). The CD4+ cells interact with MHC II and CD8+ interacts with MHC I, to aid in antigen presentation. A balance between $\mathrm{CD} 4+$ and $\mathrm{CD} 8+$ is essential for the normal functioning of the immune system (Abbas et al., 2007). 


\begin{tabular}{|c|c|c|c|c|c|}
\hline \multirow[b]{2}{*}{ Types of effector T cell } & CD8 cylotoxicT cells & $\mathrm{CO}^{\mathrm{T}} \mathrm{T}_{\mathrm{H}} 1$ cells & $\mathrm{CD}^{\mathrm{T}} \mathrm{T}_{H} 2$ cells & $\mathrm{CDAT}_{\mathrm{H}} 17$ cells & $\begin{array}{l}\text { CD4 regulatory T cells } \\
\text { (various types) }\end{array}$ \\
\hline & & & & & \\
\hline $\begin{array}{l}\text { Main functions in adaptive } \\
\text { immune response }\end{array}$ & Kill virus-infected cells & $\begin{array}{c}\text { Activate infected } \\
\text { macrophages } \\
\text { Provide help to B cells } \\
\text { for antibody production }\end{array}$ & $\begin{array}{l}\text { Provide help to } \\
\text { B cells for antibody } \\
\text { production, especially } \\
\text { switching to lgE }\end{array}$ & $\begin{array}{l}\text { Enhance } \\
\text { neutrophil } \\
\text { response }\end{array}$ & $\begin{array}{l}\text { Suppress } \\
\text { T-cell } \\
\text { responses }\end{array}$ \\
\hline Pathogens targeted & $\begin{array}{c}\text { Viruses } \\
\text { (e.g. influenza, } \\
\text { rabies, vaccinia) } \\
\text { Some intracellular } \\
\text { bacteria }\end{array}$ & $\begin{array}{l}\text { Microbes that persist in } \\
\text { macrophage vesicles } \\
\text { (e.g. mycobacteria, } \\
\text { Listeria, } \\
\text { Leishmania donovani, } \\
\text { Pneumocystis carinii) } \\
\text { Extracellular bacteria }\end{array}$ & $\begin{array}{l}\text { Helminth } \\
\text { parasites }\end{array}$ & $\begin{array}{l}\text { Extracellular } \\
\text { bacteria } \\
\text { (e.g. Salmonella } \\
\text { enterica) }\end{array}$ & \\
\hline
\end{tabular}

Table 1.1. The table shows different T cell surface receptors and depicts Th1, Th2 and Th17 pathways of CD4+ Cells. Janeway et al., 2007. 


\subsubsection{B-lymphocyte overview}

The B-lymphocytes can become either memory cells, or more likely plasma cells. The plasma cells pump out secretory antibodies $(\mathrm{Ab})$ which has the same antigen binding specificity as parent B cell (Manz et al., 1997; Slifka et al., 1998). Antibodies and $\mathrm{T}$ - cell receptors are responsible for the specificity of an immune response. The Abs either occur as soluble molecules in plasma called as immunoglobins or just attached to the surface of B cells. There are different types of antibody isotypes. They are useful in neutralization of and phagocytosis of pathogens.

To suppress infection or foreign pathogens, binding of the $\mathrm{B}$ cell receptor to the antigen is not sufficient, T-helper cells also activate antibody production. For this to occur, co-stimulation by T-helper cells, cytokines and ligand-receptor (MHC II) interactions is essential (Ochsenbein et al., 2000). These interactions result in the formation of antibodies that can help in neutralization of microbes, virus, and phagocytosis of pathogens.

\subsubsection{Natural killer cells overview}

Natural killer cells were initially discovered by their potential to act on tumor cell lines (Trinchieri, 1989). Natural killer cells are distinct from T-lymphocytes in that they do not have a TCR and CD3 receptor complex, so they were grouped as non-T cells (Biron et al., 1987; Trinchieri et al., 1989; Lanier et al., 1992; Wang et al., 1994). The NK cells protect our body from various intracellular bacteria like Listeria monocytogens, protozoal, viral infections and carcinomas (Scott P at al., 1995; Biron, 1997; Scharton- Kersten and Sher, 1997). The NK cells play an important role in the innate immune system (Santoli et al., 1978; Gidlund et al., 1978). Studies after this 
have shown that natural killer cells produce microbicidal and immunoregulatory cytokines like IL4,1L12, 1L5 and 1L 10 (Table. 1; Scharton and Scott, 1993; Warren et al., 1995; Biron and Gazzinelli., 1995; Kim and Yokoyama., 1998).

Proinflammatory cytokines TNF $\alpha$, and IL $1 \beta$ act together with NK cells in innate immunity (Figure. 1.1; Abbas et al., 2007). 


\section{CHAPTER III}

\section{LACK OF ADAPTATION TO FESCUE TOXICOSIS UNDER THERMONEUTRAL AND HEAT STRESS CONDITIONS}

\section{Abstract}

Intake of endophyte-infected tall fescue reduces feed intake, activity, and body weight gain. This study determined the potential for short-term adaptation to fescue toxicosis and heat stress. Male CD outbred rats $(n=24)$ were implanted with temperature transmitters (Respironics, Bend, OR) to measure core temperature (Tcore). In addition, feed intake (FI) and body weight (BW) were measured daily to examine three basic responses associated with fescue toxicosis using this model. Experimental design included several shifts (7 days each) in treatment to detect adaptation. All rats were initially fed diets with ground, uninfected tall fescue seed (E-) and exposed to $21^{\circ} \mathrm{C}$ (thermoneutral; TN) to establish baseline values. In Period 1, all groups were maintained at TN for 7 days, with one group fed a diet containing ground, endophyte-infected tall fescue seed (E+; 165 $\mu \mathrm{g} \mathrm{EV/kg} \mathrm{BW/d)} \mathrm{and} \mathrm{two}$ groups fed E- diet. This was followed by 7 days at $31^{\circ} \mathrm{C}$ (heat stress; HS; Period 2) on the same diets. All animals were fed E- diet during the second 7 days of HS (Period 3). In the final 7 days (Period 4), E+ diet was returned to the original group and fed to one previously E- group, with the third group remaining on E- diet. In Period 1, FI decreased by $40 \%$ with $\mathrm{E}+$ treatment $(\mathrm{P}<0.05)$, with a comparable reduction in $\mathrm{BW}$ $(\mathrm{P}<0.05)$ after 4 days. Initial HS (Period 2$)$ immediately reduced FI in all groups $(\mathrm{P}$ $<0.05)$, and diminished differences between E+ and E- groups. Growth stopped in controls, and continued to decrease in $\mathrm{E}+$ rats during HS. A shift from $\mathrm{E}+$ to $\mathrm{E}$ - diets (Period 3) immediately returned FI and average daily gain to control levels. Treatment 
of the original $\mathrm{E}+$ and one control group with $\mathrm{E}+$ diet (Period 4) produced identical reductions in FI and $\mathrm{BW}(\mathrm{P}<0.05)$ to indicate that these parameters had not adapted to fescue toxicosis. An expected reduction in daily Tcore with initial E+ treatment occurred at TN $(\mathrm{P}<0.05)$, followed by an increase during early $\mathrm{HS}(\mathrm{P}<0.05)$. Removal of $\mathrm{E}+$ in Period 3 produced a reduction in daily Tcore $(\mathrm{P}<0.05)$ that coincided with increase in FI. Both groups fed E+ in Period 4 exhibited similar Tcore responses with no increase from Period 3. Although feed intake and growth shows no sign of adaptation to fescue toxicosis, there is indication that adaptation to heat stress improves the thermal response.

\section{Introduction}

A large portion of the tall fescue (Lolium arundinaceum) in the US contains an endophyte (Neotyphodium coenophialum) that benefits the plant, but causes problems in animals that are collectively known as fescue toxicosis. The most common symptoms are decreased feed intake and an associated reduction in body weight gain. Peripheral vasoconstriction, overgrowth of winter coat, and hyperthermia during heat stress are additional signs associated with fescue toxicosis (Strickland et al., 1993). Binding of fescue toxins to adrenergic and serotonergic receptors are considered major reasons for clinical signs in animals (Oliver, 2005). A rodent model has been developed in our laboratory with evaluated responses that resemble those from bovine models of fescue toxicosis, that allows for rapid analysis of many components of this condition (Spiers et al., 2005). Classical studies show that animals are able to adapt to numerous stressors such as heat and cold conditions and, in turn, become more tolerant of these conditions. There are no controlled studies that demonstrate adaptation to ergot alkaloids and heat stress associated with fescue toxicosis. We 
hypothesize that sensitizing rats to these collective stressors might help them maintain lower body temperature, with improved feed intake and growth, on subsequent exposures.

\section{Materials and methods}

\section{Animals and treatment}

Adult, CD outbred, male rats (400 to $500 \mathrm{~g} \mathrm{BW}$; $=24$ ) were implanted with a transponder (G2 E-Mitter; Respironics, Bend, OR) to measure core body temperature $\left(\mathrm{T}_{\text {core }}\right)$ every 10 minutes throughout the study period Data loggers (HOBO Boxcar Prodata; Onset Computer Corp., Bourne, MA) were placed in the chamber to record ambient temperature and percent relative humidity throughout the study. A xylazine $(13 \mathrm{mg} / \mathrm{ml})$ and ketamine $(87 \mathrm{mg} / \mathrm{ml})$ cocktail, at the dose rate of $0.1 \mathrm{mg} / \mathrm{kg} \mathrm{BW}$, was used to anesthetize the rats. E-mitters were sutured to the body wall through midventral incision. The incision was closed using simple interrupted sutures. Animals were allowed a postoperative recovery period of one week. Treatment diet, containing endophyte-infected fescue seed $(\mathrm{E}+)$ was formulated to provide approximately $165 \mu \mathrm{g}$ ergovaline/kg BW/d using standard protocol (Rottinghaus et al., 1993). Control diet, which contained uninfected fescue seed, did not have a significant level of ergovaline. Diets were stored at $4^{\circ} \mathrm{C}$ prior to feeding rats. All procedures were approved by University of Missouri Animal Care and Use Committee.

\section{Treatment period and sample collection}


After one week of postoperative recovery, all animals were initially maintained at thermoneutrality $\left(\mathrm{TN} ; 21^{\circ} \mathrm{C}\right)$ for seven days and then exposed to a constant heat stress (HS; $31^{\circ} \mathrm{C}$ ) for 21 days (Figure. 1.3 ). Relative humidity was maintained at $50 \pm 5 \%$ during this period. Rats were assigned to one of three treatment groups during the recovery period. They consisted of: 1) Control (C) rats that received E- diet throughout the entire study, 2) Exposure 1 (Exp. 1) rats that received E+ diet for two weeks, followed by one week on E- diet, and a final week on E+ diet, and 3) Exposure 2 (Exp. 2) rats that received E- diet for three weeks, followed by a final week on E+ diet. Feed composition of treatment and control diets was the same as in previous trials (Spiers et al., 2005). Water and feed were provided for ad libitum intake throughout the study. Feed intake and body weight were measured daily at 1000. At the end of the study, rats were anesthetized using a xylazine $(13 \mathrm{mg} / \mathrm{ml})$ and ketamine $(87 \mathrm{mg} / \mathrm{ml})$ cocktail at the dose rate of $0.1 \mathrm{mg} / \mathrm{kg} \mathrm{BW}$. Blood was collected from anesthetized rats using intracardiac puncture.

\section{Statistical analysis}

Daily averages were calculated for all data by day (FI and BW) and hour by day (Tcore) using a two-way analysis of variance (ANOVA; JMP ${ }^{\circledR} 8$; SAS Institute, Inc.). All parameters were analyzed for significant effects using repeated measures ANOVA of SAS and a standard least square model fit (JMP ${ }^{\circledR} 8$; SAS Institute, Inc.) to ascertain time-related changes in these parameters. Model components included treatment, time, and animal as class variables to determine overall treatment and time-related differences, and treatment by time interactions. Once a significant difference was noted for any parameter, a Si Tukey-Kramer multiple comparison test (Steele and Torrie, 1980) was performed to identify specific differences $(\mathrm{P}<0.05)$. A P value of 
less than or equal to 0.05 was reported as significant. Linear plot analysis was performed using the mean weight of all rats in a treatment group for a particular day.

\section{Results}

\section{Feed intake}

Rats fed the E- diet during the pretreatment period (Days -5 to 7; Figure. 1.4) exhibited no diet $(\mathrm{P}>0.71)$ or diet by day effects on feed intake $(\mathrm{FI})(\mathrm{P}>0.96)$. In contrast, FI decreased by $40 \%$ on the first day (Day 8) of E+ treatment (Exp. 1; P $<$ 0.05) in Period 1 compared to the pretreatment period (Day 7). A significant diet by day effect $(\mathrm{P}<0.0001)$ was also observed from Days 8 to14 at TN. Rats fed the E+ diet (Exp. 1) had a lower FI, compared to E-TN rats until Day 14. From Days 8 to 15, the FI of the E+ rats was lower than that of rats fed the E- diet. Exposure to HS (Days 15 to 36$)$ produced reduction $(\mathrm{P}<0.05)$ in FI for all treatment groups compared to preheat levels (Figure. 1.2, Period 2). A 35\% reduction in FI of E+ rats occurred from Days 14 to. The FI of both E-HS groups was reduced $37 \%$ on the first day of HS. However, these rats showed signs of adaptation with an increase in FI $(\mathrm{P}<0.05)$ of $30.76 \%$ from Day 15 to 16 , where it stabilized. Feed intake increased within 24 hours in Period $3(\mathrm{P}<0.05)$ when rats were switched from E+ to E- diets (Days 21-28). The Exp. 1 rats increased FI by $47 \%$ when returned to E- diet. There were no treatment differences in FI in Period $3(\mathrm{P}>0.17)$. Return to $\mathrm{E}+$ diet in Period 4 produced a reduction $(\mathrm{P}<0.05)$ in $\mathrm{FI}$ of the Exp. 1 rats when $\mathrm{E}+$ diet was reintroduced (Exp.1, Period 4) or presented to the Exp. 2 group for the first time. During this period, there was a significant diet $(\mathrm{P}<0.0001)$ and diet by day effect $(\mathrm{P}<0.0001)$. For Exp. 1 
rats, FI decreased by $56.21 \%$ from Days 28 to 29 . In contrast, the rats receiving E+ diet for the first time (Exp. 2) reduced FI by 36.6\% during this period.

\section{Body weight gain}

As expected there was no diet effect $(\mathrm{P}<0.95)$ on body weight during the pretreatment period (Days 5 to 7$)$. However, there was a day effect $(\mathrm{P}<0.0001)$ due to normal daily weight gain. A significant day effect $(\mathrm{P}<0.0001)$ was observed when rats were fed the E+ diet in Period 1. Body weight gain was reduced in rats on the $\mathrm{E}+$ diet (Exp. 1) after 3 days at TN (Figure. 1.5, Period 1; $\mathrm{P}<0.05$ ), while the other groups were unaffected. A linear regression plot from Days 8 to 14 showed a positive weight gain for rats in Control and Exp. 2 groups $(2.06 \mathrm{~g} / \mathrm{d}$ and $1.68 \mathrm{~g} / \mathrm{d}$, respectively). In contrast, Exp. 1 rats lost body weight at a rate of $2.5 \mathrm{~g} / \mathrm{d}(\mathrm{P}<0.05)$ during this period.

Although all treatment groups exhibited a reduction in body weight in Period 2 with exposure to HS (Days 15 to 21, Figure. 3), there was a greater reduction in rats on the $\mathrm{E}+\operatorname{diet}($ Exp. 1). Also, there was a significant day effect $(\mathrm{P}<0.0001)$. Body weight was lowest for all groups during the first two days of HS, followed by a gradual increase for only the rats on the E- diet. A linear plot showed that Control ($1.7 \mathrm{~g} / \mathrm{d})$, Exp. $1(-3.2 \mathrm{~g} / \mathrm{d})$ and Exp. 2 rats $(-1.6 \mathrm{~g} / \mathrm{d})$ lost weight $(\mathrm{P}<0.05)$ throughout this period.

Change from E+ to E- diets (Days 22-28, Figure. 1.5, Period 3.; Exp. 1) produced a return in growth rate within 2 days, even under HS conditions. During this period, there was a significant day effect $(\mathrm{P}<0.0001)$. A linear plot revealed a daily weight gain $(\mathrm{P}<0.05)$ in Control $(1.8 \mathrm{~g} / \mathrm{d})$, Exp. $1(2.6 \mathrm{~g} / \mathrm{g})$ and Exp. $2(0.60 \mathrm{~g} / \mathrm{d})$ treatment groups. 
The second exposure of rats to E+ during HS (Days 29-36; Period 4; Exp. 1) produced significant day $(\mathrm{P}<0.0001)$ and day by diet effects $(\mathrm{P}<0.0001)$. Rats in both $\mathrm{E}+$ groups in Period 4 displayed the same reduction in growth rate compared to the control group. Linear slope values showed weight change in Control $(1.81 \mathrm{~g} / \mathrm{d})$ rats, and significant weight loss $(\mathrm{P}<0.05)$ in Exp. $1(-3.31 \mathrm{~g} / \mathrm{d})$ and Exp. 2 rats $(-3.94$ $\mathrm{g} / \mathrm{d})$.

\section{Core body temperature}

\section{Average daily core body temperature}

Average hourly values are shown in Figure 4 for rats in the three treatment groups during the entire period of study. It can be seen that there are both E+ and thermal effects across the four periods of the study that require more detailed analysis. There was no difference across treatment groups during the TN adjustment baseline period. In addition, there was no diet $(\mathrm{P}>0.87)$ or diet by day effects $(\mathrm{P}>0.42)$ during this period. Once the rats were placed on E+ diet at TN (Exp. 1; Period 1) there was an overall reduction in $\mathrm{T}_{\text {core }}\left(0.22^{\circ} \mathrm{C} ; \mathrm{P}<0.05\right.$; Figure. 1.6$)$ below rats on the E- diet (Figure. 1.7). During this period, there were significant day effect $(\mathrm{P}<$ 0.0001), but not a diet effect $(\mathrm{P}<0.21)$. The lowest $\mathrm{T}_{\text {core }}$ was recorded on Day 13, which was five days after the start of E+ diet treatment.

In contrast to the $\mathrm{TN}$ response, intake of $\mathrm{E}+$ diet during HS (Exp. 1; Period 2; Figure. 1.7) produced an increase in $\mathrm{T}_{\text {core }}\left(0.30^{\circ} \mathrm{C} ; \mathrm{P}<0.05\right)$ above the level for rats on the E- diet. Maximum $\mathrm{T}_{\text {core }}$ for the $\mathrm{E}+$ group (Exp. 1) was $0.50^{\circ} \mathrm{C}$ above preheat level on the fifth day after the start of HS. During this period, there were significant thermal, $(\mathrm{P}<0.0001)$ day, $(\mathrm{P}<0.0001)$ and diet by day effects $(\mathrm{P}<0.0001)$. 
All treatment groups in Period 3 had a higher $\mathrm{T}_{\text {core }}$ due to HS compared to pretreatment levels with no significant differences between treatment groups. No E+ effect $(\mathrm{P}>0.74)$ was observed, but there were significant day and day by $\mathrm{E}+$ effects $(\mathrm{P}<0.0001)$ during Period 3. Subsequently, E+ diet treatment (Period 4) produced a marked increase in $\mathrm{T}_{\text {core }}(\mathrm{P}<0.05)$ of rats $($ Exp. 2$)$ receiving $\mathrm{E}+$ diet for the first time. This effect was nonexistent after several days on the E+ diet (Exp. 2), and was different from the initial HS exposure on the E+ diet (Exp. 1). This increase was not observed in rats (Figure 8, Exp. 1, Period 4) previously sensitized to the toxin or HS.

\section{Hourly core body temperature}

Hourly $\mathrm{T}_{\text {core }}$ for the pretreatment period (Days 1 to 7 ) showed expected circadian changes. Both hour $(\mathrm{P}<0.0001)$ and hour by treatment $(\mathrm{P}<0.0001)$ effects were observed. The highest $\mathrm{T}_{\text {core }}$ was at 0000 to 0200 , and corresponded to the time of day when rats are most active. A significant decrease $(P<0.05)$ in $T_{\text {core }}$ was observed from 0600 to 0700 before lights were turned on. Similarly, $\mathrm{T}_{\text {core }}$ started to increase after 1800 when lights were turned off. These findings are similar to previous reports in the rodent model (Gordon, 1993). The spike in $\mathrm{T}_{\text {core }}$ at 1000 and 1200 is an artifact associated with daily feeding time, and is attributable to increased activity and caloric intake.

Rats fed the E+ diet at thermoneutrality experienced a hypothermic response (Exp. 1; Period 1; Figure. 1.8) that was most evident at 0900 to $1000(\mathrm{P}<0.05)$ when the nocturnal rat reaches its lowest point in its circadian rhythm (Days 11-14). Other effects were similar to pretreatment levels.

Rats exposed to heat stress had a similar circadian $\mathrm{T}_{\text {core }}$ pattern to pretreatment level, but only at a higher level (Days 16 to 21). Heat stress hyperthermia (Exp. 1; 
Period 2; Figure. 1.9) associated with fescue toxicosis was significant at 0500 to 0700 $(\mathrm{P}<0.05)$, when the rat exhibits the increase in core temperature of the daily circadian cycle,

Circardian variation in Period 4 (Figure. 1.10) showed that rats in Exp. 2 had a large spike in $\mathrm{T}_{\text {core }}$ as it was exposed to the toxin for the first time even after adapting to HS (Days 30 and 31; Figure. 1.10). These effects were maximal $(\mathrm{P}<0.05)$ from 0900 to 1900 when lights were on. However, after three days the rats exposed to the toxin for the first time had adapted with a $\mathrm{T}_{\text {core }}$ only slightly above Exp 1 level (Figure. 1.11).

\section{Discussion}

Rats fed an E+ diet during heat stress had shifts in FI and growth that changed over time. Likewise, $\mathrm{T}_{\text {core }}$ was altered over time under these conditions (Figure. 4) that reflected adaptive shifts in thermal status.

\section{Feed intake}

Feed intake was reduced by $40 \%$ within 24 hours following the initial intake of an $\mathrm{E}+$ diet at TN. Reduction in feed intake is a sensitive marker of fescue toxicosis. More than 50\% reduction in feed intake of rats was observed in previous studies when animals were fed an endophyte-infected fescue seed diet (Roberts et al., 2002; Spiers et al., 2005; Al-Tamimi et al., 2007; Settivari et al., 2008). Previous studies have shown that FI of E+ treated rats gradually improves over time under both thermoneutral and heat stress conditions (Spiers et al., 2005). These effects were evident from Day 4 in the current experiment, but they did not reach the levels of control animals until the end of the study period. A similar pattern of recovery from 
Day 4 at thermoneutrality has also been reported by Roberts et al.(2002). Decreasing feed intake helps rats lower core body temperature when subject to heat stress (Shido et al., 1991; Gordon, 1993). The specific mechanism of recovery in FI is unknown and reasons for recovery have not been established.

The feed intake of E-HS rats was reduced $37 \%$ on the first day of heat stress. However, control rats showed signs of adaptation and their feed intake increased $30 \%$ after two days in heat. These findings are similar to previous studies of rats (Johnson and Stack, 1989; Spiers et al., 2005). Similar to rats, cattle also showed a significant decrease in feed intake immediately after exposure to heat stress (Wallace et al., 1996). A 10 to $25 \%$ drop in feed intake was observed in cattle on the first day of exposure to heat stress at $29^{\circ} \mathrm{C}$ (Kibler et al., 1965). This suggests the rat model follows a pattern similar to cattle in reduction of feed intake. Reduced FI may be due to decreased duration, frequency and quantity of FI (Johnson and Cabanac., 1982; Johnson and Strack., 1989). Al- Tamimi et al. (2007) stated that a decreased FI is an adaptive mechanism to lower post prandial heat production.

Feed intake in all rats increased when switched from E+ diet to E- diet. Recovery of feed intake occurred within $24 \mathrm{~h}$ with a switch from $\mathrm{E}+$ to $\mathrm{E}$ - diets (Figure. 1.4, Period 3; Exp. 1). The Exp. 1 rats had an increase in FI of 47\% when returned to E- diet, which was equal to E- control level. This experimental protocol involving removing and reintroducing endophyte in rat diets has not been previously used. However, past studies have clearly shown that the effect of endophyte and heat stress causes a greater decrease in feed intake compared to heat stress alone in cattle, lamb, and rat models used to study fescue toxicosis (Osborn et al., 1992; Gadberry et al., 2003; Spiers et al., 2005). This is the first time that this type of study is done as earlier studies have not attempted to study effects sensitizing rats to the toxin. 
This study demonstrates that feed intake was reduced in response to heat stress or intake of endophyte-infected fescue seed, with a greater decrease occurring with the combination of both stressors (Figure. 1.4). These results are consistent with previous studies using rats (Osborn et al., 1992; Spiers et al., 2005; Al-Tamimi et al., 2007). Spiers et al. (2005) also reported a gradual recovery of feed intake when rats were continuously fed the E + diet. However, they did not examine recovery and adaptation by removing them from the E+ diet followed by re exposure. In the present study, it was clear that repeated intake of the $\mathrm{E}+$ diet did not help the rats to maintain a higher feed intake when taken off the $\mathrm{E}+$ diet and then returned to it again (Figure. 1.4). Spiers et al. (2005) suggested that this reduction in FI is not due to palatability of the diet. A lack of adaptation in feed intake could have a significant economic impact if similar effects are seen in cattle, as reported in earlier studies (Hemken et al., 1981; Osborn et al., 1992)

\section{Body weight}

A reduction in body weight occurred in the present study in response to intake of the $\mathrm{E}+$ diet, heat stress, and the combination of both. The greatest effect was observed with the combination (Figure. 1.5). These results are consistent with previous results (Spiers et al., 2005; Al-Tamimi et al., 2007). Average daily body weight gain is reduced in the same pattern as feed intake, however it takes considerably longer for weight to recover compared to feed intake. Past studies reported a $63 \%$ decrease in average daily gain at thermoneutrality when rats were fed an endophyte-infected diet compared to those fed an uninfected diet (Neal and Schmidt, 1985). Similarly, a decrease of 22 to $79 \%$ in body weight was observed in 
cattle, when fed with endophyte-infected diet at thermoneutrality (Schmidt et al., 1982; Stuedemann et al., 1986).

Rats fed $\mathrm{E}+$ diet in heat stress experienced a greater reduction in body weight ($3,2 \mathrm{~g} / \mathrm{d})$ compared to control rats $(-1.6 \mathrm{~g} / \mathrm{d})$. This is in agreement with previous studies using rats (Roberts et al., 2002) and sheep (Gadberry et al., 2003). Spiers et al. (2005) also reported a greater reduction in body weight of E+HS rats compared to E-HS rats. In this earlier study, E+HS rats never reached the body weight of E-HS rats.

When rats were switched from the $\mathrm{E}+$ to the $\mathrm{E}$ - diet in the heat there was an immediate recovery in body weight gain $(2.6 \mathrm{~g} / \mathrm{d})$, but they did not reach the levels of control rats which had never received the $\mathrm{E}+$ diet. This is in agreement other studies of heat stress response in rats (Johnson and Cabanac, 1982; De Vries et al., 1993). Rats in Exp. 1 and Exp. 2 groups decreased their feed intake in Period 4 and, as a result, showed similar decreases in body weight. This experiment shows that there is no significant adaptation of the body weight gain response to intake of endophyteinfected fescue (Figure. 1.5). Similar studies of adaptation to intake of the E+ diet in the heat have not been done.

Recent genomic analysis in our laboratory has shown that genes related to protein catabolism and gluconeogenesis were upregulated at thermoneutrality (Settivari et al., 2006). Even if rats started eating more, the effect of ergopeptine alkaloids on the mitochondrial genes corresponding to ATP may prevent them from gaining weight and reaching body weights of E- rats (Settivari et al., 2006). Similar effects in beef cattle could cause heavy losses to farmers and sensitizing cattle to the toxin may not help them improve their body weight gain. 


\section{Core body temperature}

Rats in the present study on the E+ diet exhibited both hypothermic and hyperthermic periods that were associated with the thermal environment. Consistent with previous studies (Settivari et al., 2006; Spiers et al., 2005 ), thermoneutral hypothermia $\left(0.22^{\circ} \mathrm{C}\right.$ reduction on the first day of exposure to $\mathrm{E}+$ at $\left.\mathrm{TN}\right)$ was observed when rats in the present study were fed the E+ diet at $21^{\circ} \mathrm{C}$. By Day 7 on the $\mathrm{E}+$ diet the $\mathrm{T}_{\text {core }}$ of these rats did not differ significantly from controls. This is supported by previous reports which state that rats (Settivari et al., 2006) and cattle (Osborn et al., 1992) experiencing fescue toxicosis recover from thermoneutral hypothermia.

When exposed to heat stress all groups increased $\mathrm{T}_{\text {core }}$ within 24 hours. However, a greater increase was observed in rats fed the $\mathrm{E}+$ diet compared to rats fed the Ediet. This is in agreement with other rat studies (Settivari et al., 2006; Spiers et al., 2005). Likewise, there are similar increases in $T_{\text {core }}$ response to heat stress and fescue toxicosis in cattle and sheep (Hoveland, 1983; Osborn et al., 1992; Gadberry et al., 2003). In the present study, heat stressed rats showed signs of adaptation after two days in the heat, and returned to preheat levels. This is in agreement with previous reports, which showed adaptation of rats to heat stress (Matthew, 1997). Both heat loss and production avenues can be adjusted as a means to lower $\mathrm{T}_{\text {core }}$. Adaptive heat lose mechanisms would include increased splanchnic and peripheral vasodilation for increased convective loss (Horowitz, 1993). Likewise, evaporative cooling could be increased by increasing surface area for evaporation with limb extension, and spreading saliva (Schmidek et al., 1983, Hubbard et al., 1982; Gordon, 1993). Once heat loss mechanisms reach their upper limits, the only additional adjustment is to lower heat production. Others have proposed that reduced activity and metabolic heat 
production can be used by rats to lower heat production as adaptation to heat stress (Shido et al., 1991; Gordon, 1993). Reduction in caloric intake as seen in the present study might, in turn, lower metabolic heat production which would decrease the need for excessive heat loss and ultimately result in a decrease in $\mathrm{T}_{\text {core }}$.

The increase in core body temperature of E+HS rats above E-HS rats is attributed to the effects of ergopeptine alkaloids on the thermoregulatory system (Spiers et al., 1995). The primary action is increased peripheral vasoconstriction caused by adrenergic and dopaminergic binding of egopeptine alkaloids (Aldrich et al., 1993; Oliver., 2005). Another possible reason is the inhibition of nitric oxide activity which is needed for vasodilation in the heat to increase convective heat exchange (AlTamimi et al., 2007). The $\mathrm{T}_{\text {core }}$ of $\mathrm{E}+$ rats remained above that of $\mathrm{E}-\mathrm{HS}$ rats until they were taken off $\mathrm{E}+$ diet. Previous studies have reported that rats adapted to $\mathrm{E}+$ diet in thermoneutral and heat stress conditions (Roberts et al., 2002).

Genes corresponding to carbohydrate, lipid, and protein metabolism may be altered to help rats adapt to decreased caloric intake and increased $\mathrm{T}_{\text {core }}$ (Settivari et al., 2006). Settivari et al. (2006) reported upregulation of genes involved in gluconeogenesis, which include phosphoenolpyruvate carboxykinase and fructose-16-biphosphatase. Since, gluconeogenesis helps rats derive energy from not carbohydrate sources like stored fat, it helps rats to maintain a normal energy balance inspite of lower feed intake.

In the present study, rats showed an adaptive response of $\mathrm{T}_{\text {core }}$ when exposed to thermal stress in Period 4. Previous exposure to toxin (Period 2, Figure. 1.7) helped the rats to maintain a lower $T_{\text {core }}$ (Figure. 1.7) compared to rats that received toxins for the first time. One possible explanation from previous data in our lab, is the upregulation of CYP 450 genes in liver when exposed to the toxins for the first time, 
which may help to detoxify ergot toxins on subsequent exposure. Another new finding is the greater difference in $\mathrm{T}_{\text {core }}$ observed during the day (Figure. 1.10) when rats were exposed to heat stress and toxin for the second time. We do not know why these differences are seen during the day. Since, core body temperature shows an adaptive response a detailed analysis is needed to ascertain the physiology of adaptation and other new findings in the study.

\section{Summary}

The current study shows that there is a lack of adaptation to fescue toxicosis and heat stress for of feed intake and body weight gain. Core body temperature response to heat stress does exhibit adaptation over time. Once rats adapt to heat stress, the hyperthermic response to fescue toxicosis in the heat is diminished. Because of this adaptive response, it might be useful to expose animals to the toxin before peak summer heat to enable to provide an imporved response to fescue toxicosis and heat stress. Mortality associated with this combination of stressors might also be avoided. Future studies should be aimed at looking at other physiological parameters and genes related to the adaptive responses. 

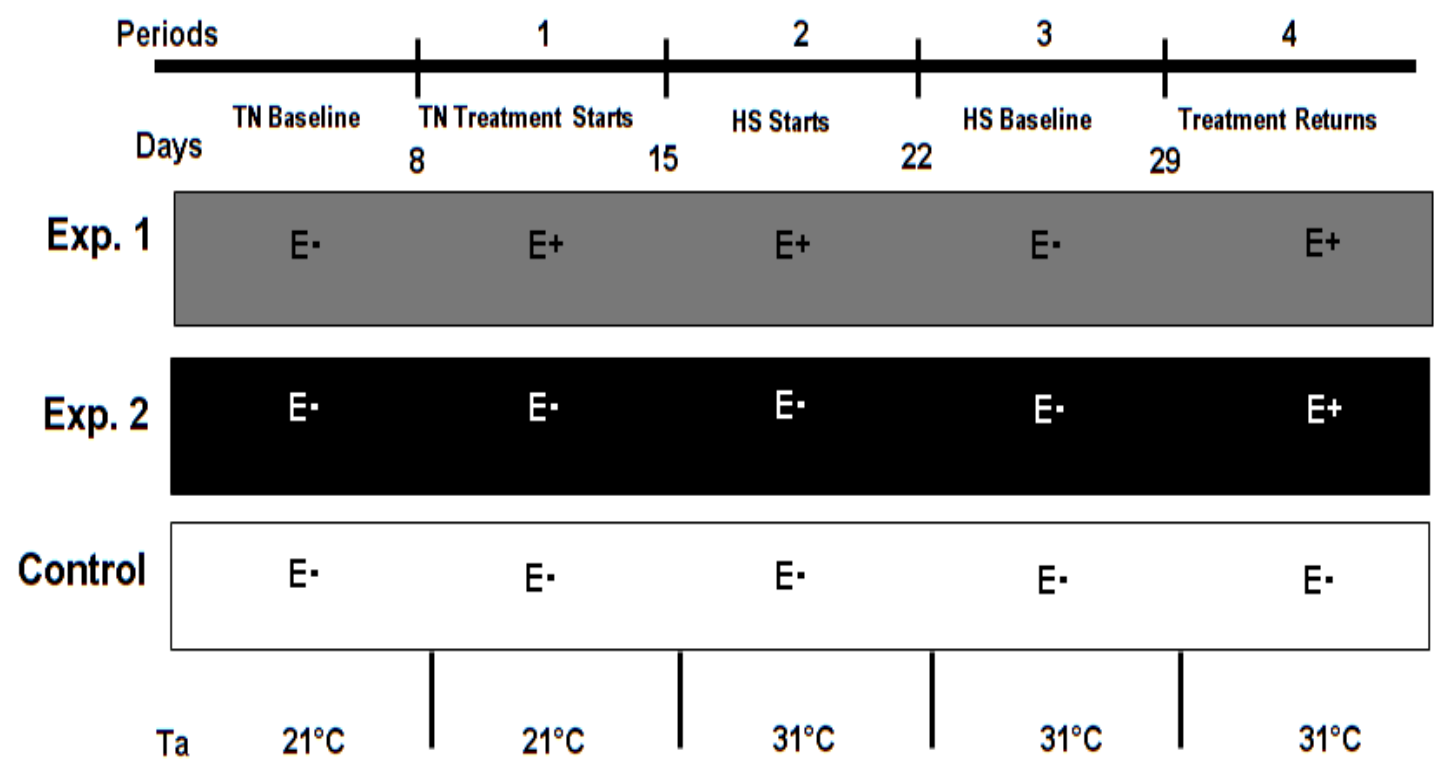

Figure 1.3. Timeline of the study showing E + and E- rats maintained in different thermal environments. The time-line also shows the number of days that rats were exposed to each of the stressors. 


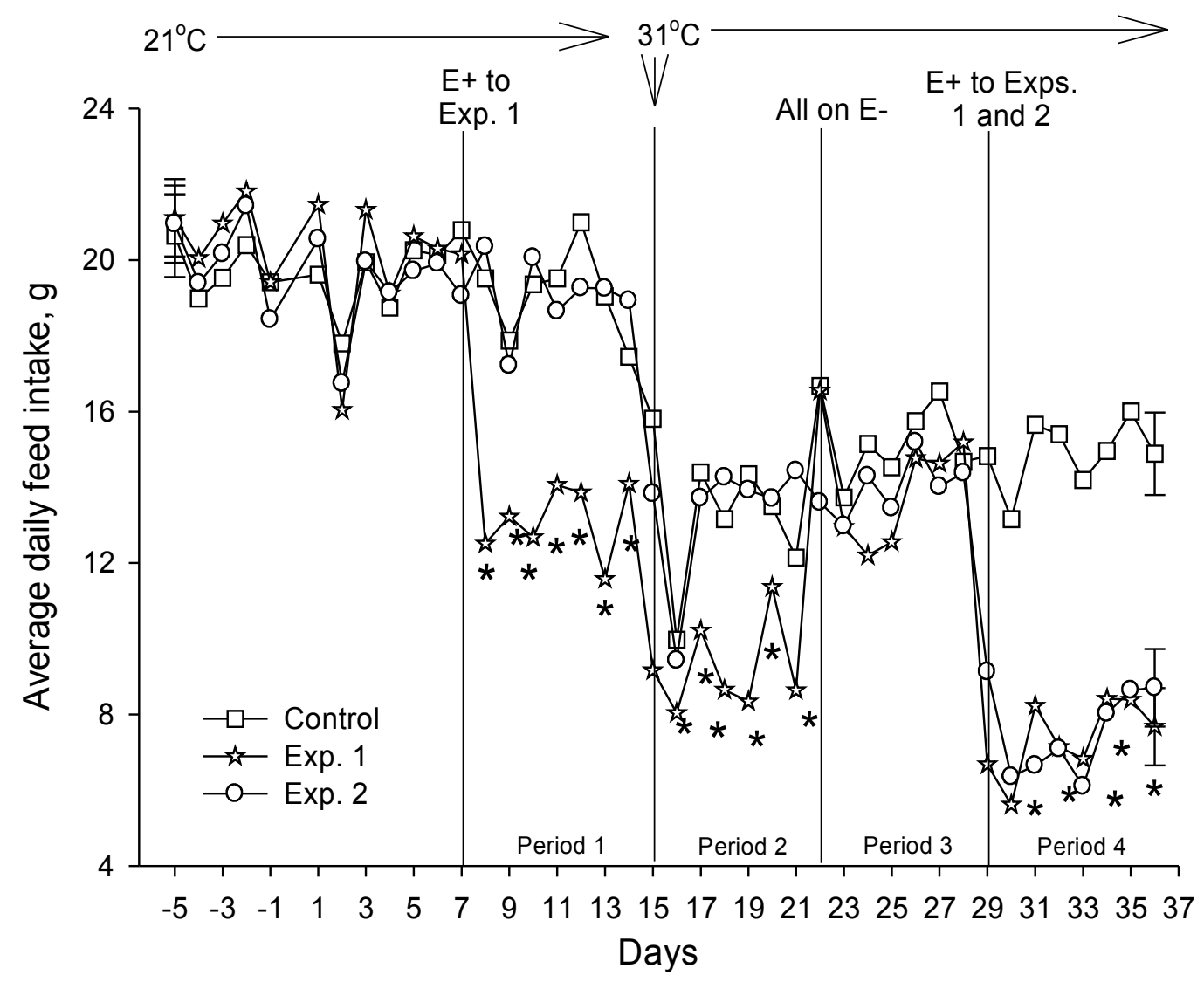

Figure 1.4. Average daily feed intake of Control, Exp. 1 and Exp. 2 groups is shown. Solid lines divide TN baseline, diet treatment at TN, HS and diet treatment, HS baseline, and return to diet treatment in the heat. An asterisk (*) indicates a significant $\mathrm{E}+$ effect compared to E- groups. The vertical lines above and below the first and last points indicate $\pm \mathrm{SE}$. 


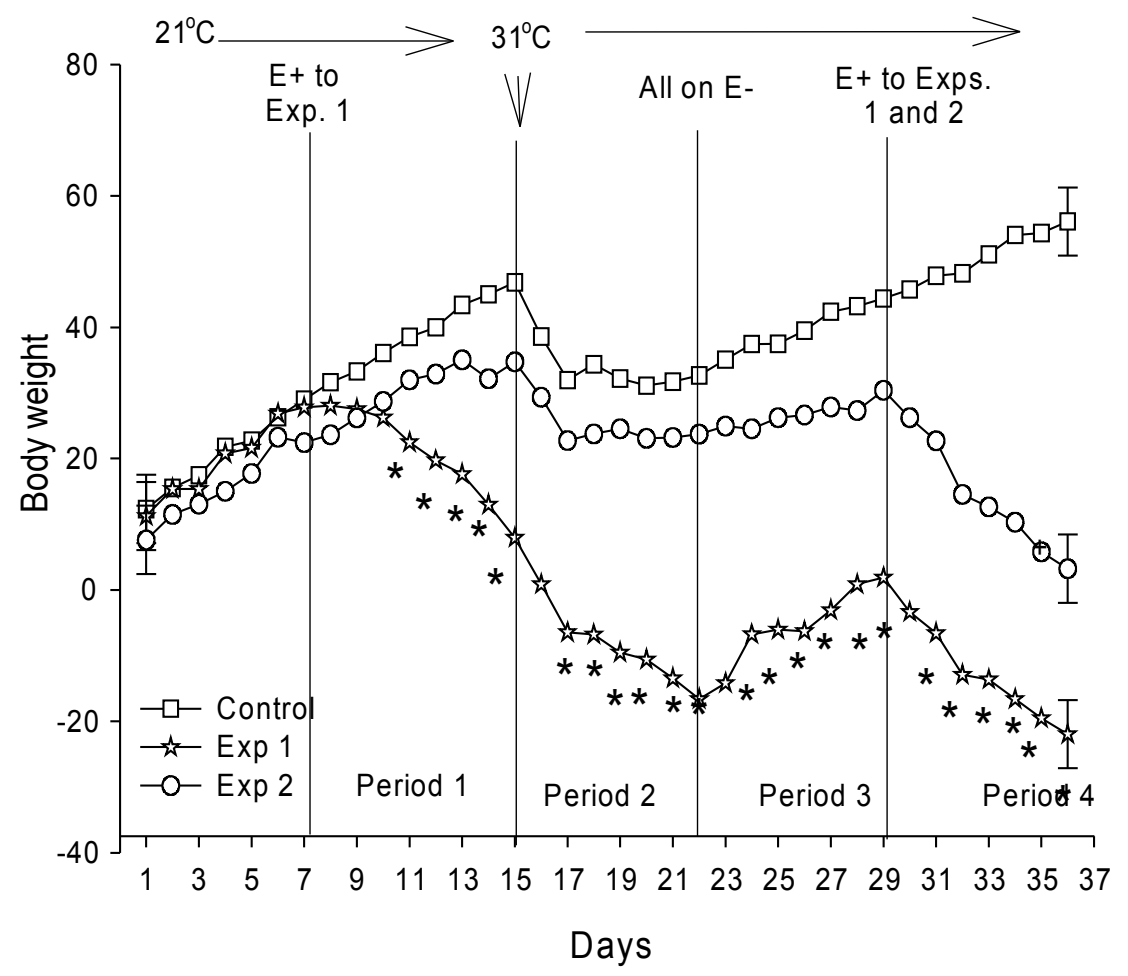

Figure 1.5. Average body weight gain of Control, Exp. 1 and Exp. 2 groups is shown. Solid lines divide TN baseline, diet treatment at TN, HS and diet treatment, HS baseline, and return to diet treatment in the heat. An asterisk $(*)$ indicates a significant $\mathrm{E}+$ effect compared to E- groups. The vertical lines above and below the first and last 


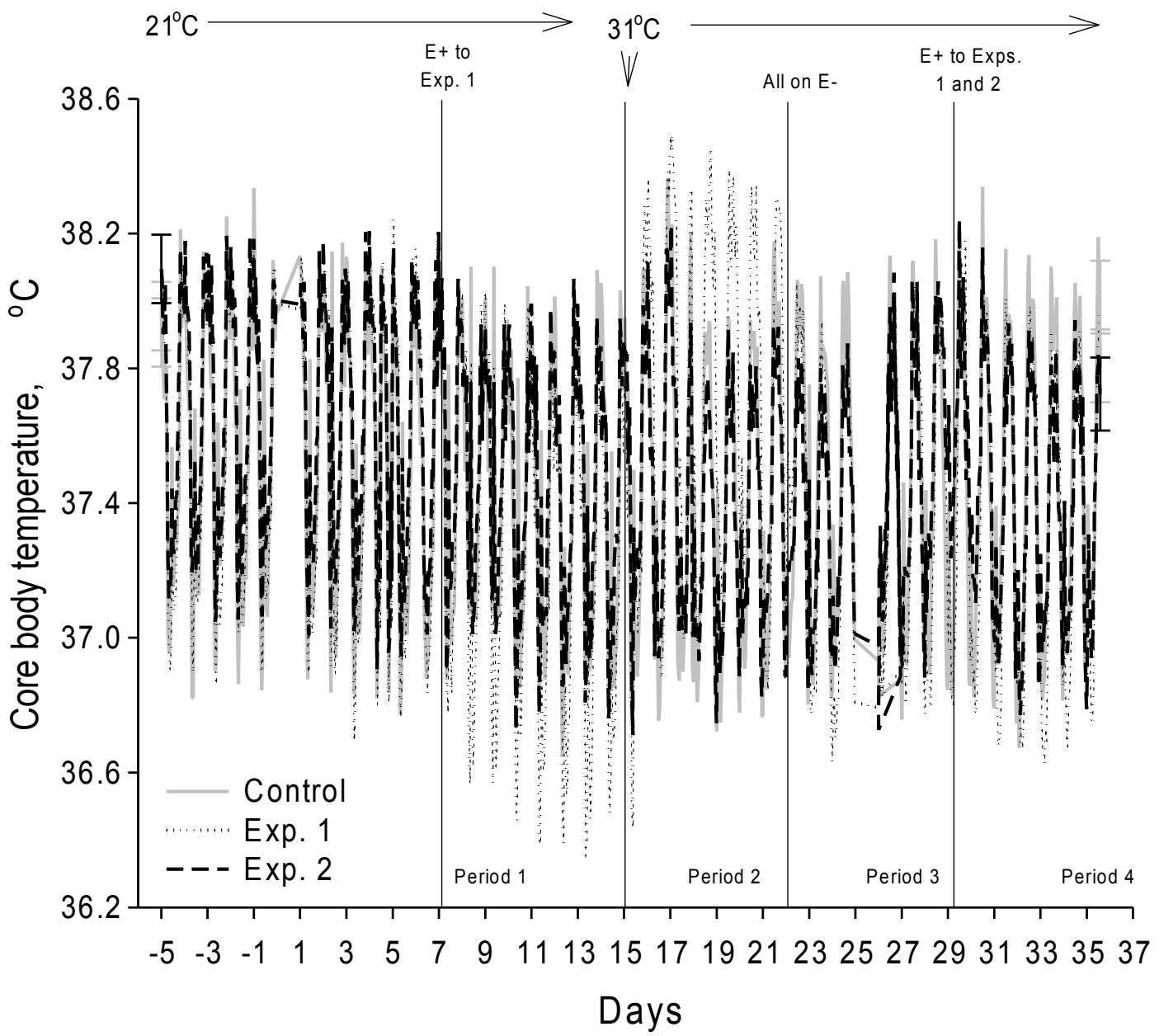

Figure 1.6. Average hourly core body temperature of rats in the Control, Exp. 1 and Exp. 2 groups over the entire study period. Solid lines divide the different periods that include in order thermoneutral baseline, diet treatment at thermoneutrality, heat stress and diet treatment, heat stress off $\mathrm{E}+$ treatment and heat stress with the return to E+ treatment. Figure shows core body temperature of all rats during entire study period with visible signs of hypothermia and hyperthermia. The vertical lines above and below the first and last points indicate \pm SE. 


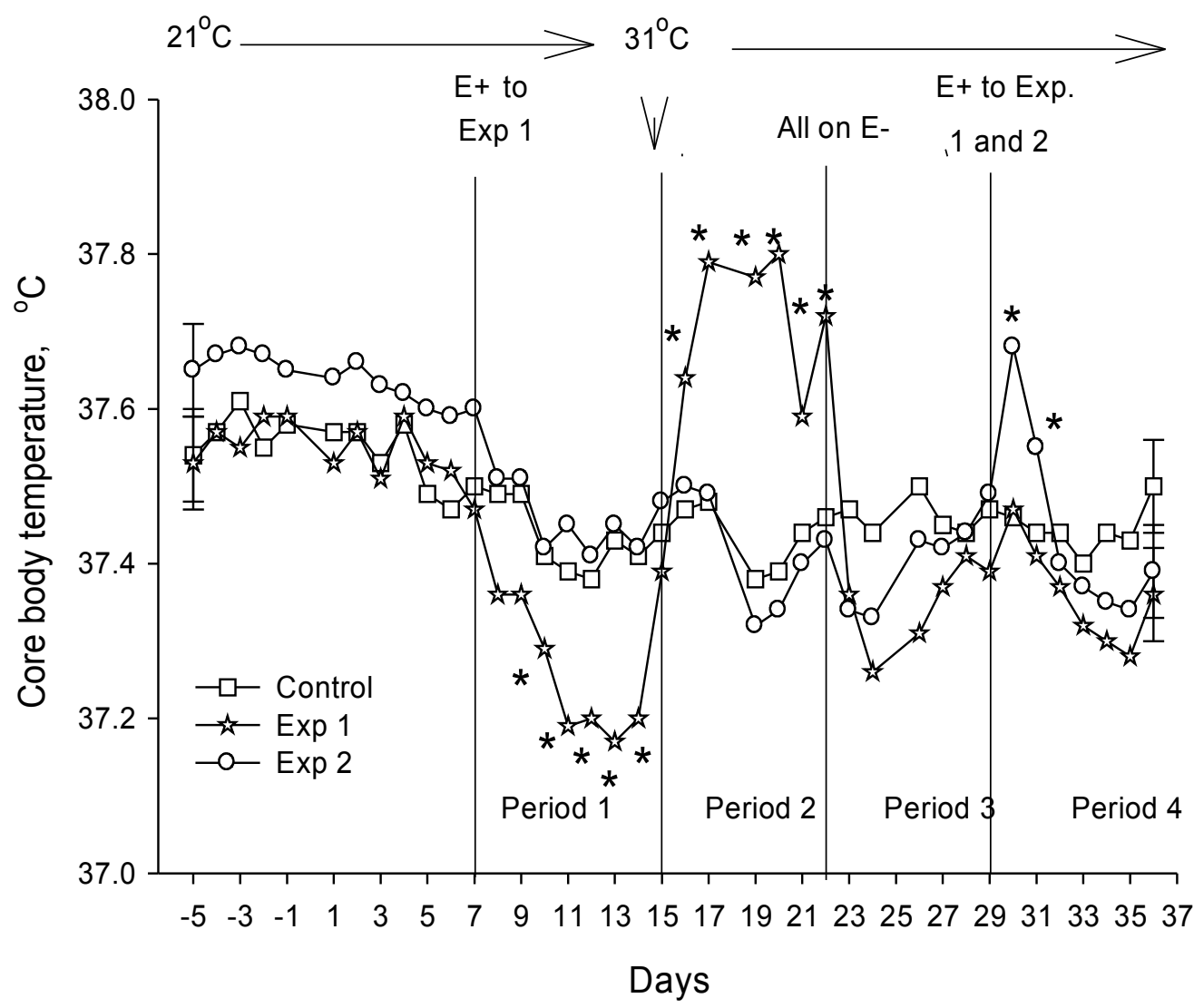

Figure 1.7. Average daily core body temperatures of rats in the Control, Exp. 1 and Exp. 2 groups. Solid lines divide the different periods that include, in order: thermoneutral baseline, diet treatment at thermoneutrality, heat stress and diet treatment, heat stress off E+ treatment, and heat stress with the return to E+ treatment. An asterisk (*) indicates a significant endophyte effect compared to endophyte free groups. Both of these effects are discussed in detail in the text. The vertical lines above and below the first and last points indicate \pm SE. 


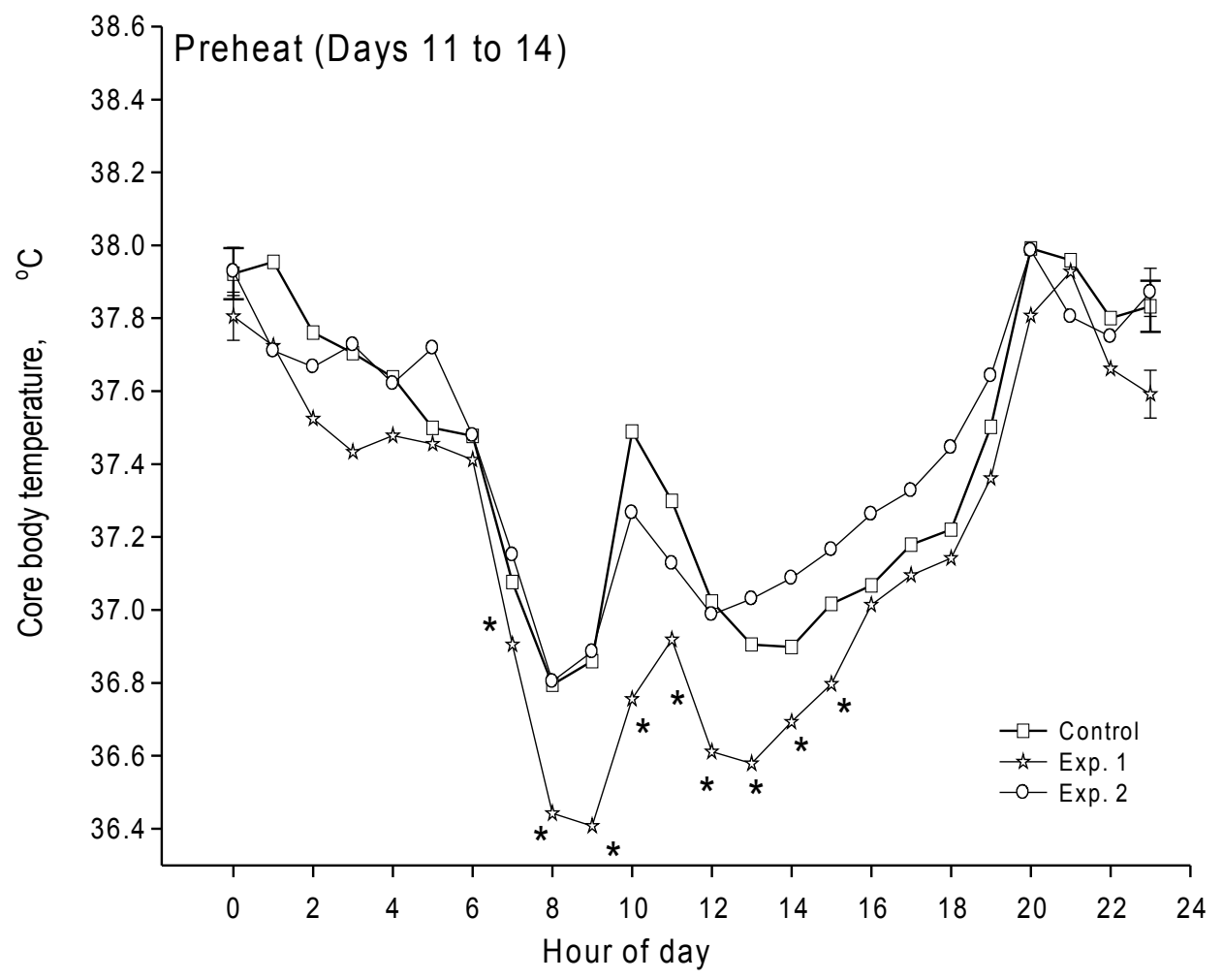

Figure 1.8. Hourly core body temperature averaged by group and averaged for days 11 to 14 for Control, Exp. 1, and Exp. 2 groups. An asterisk (*) indicates a significant difference from previous hour of day. The vertical lines above and below the first and last points indicate \pm SE. 


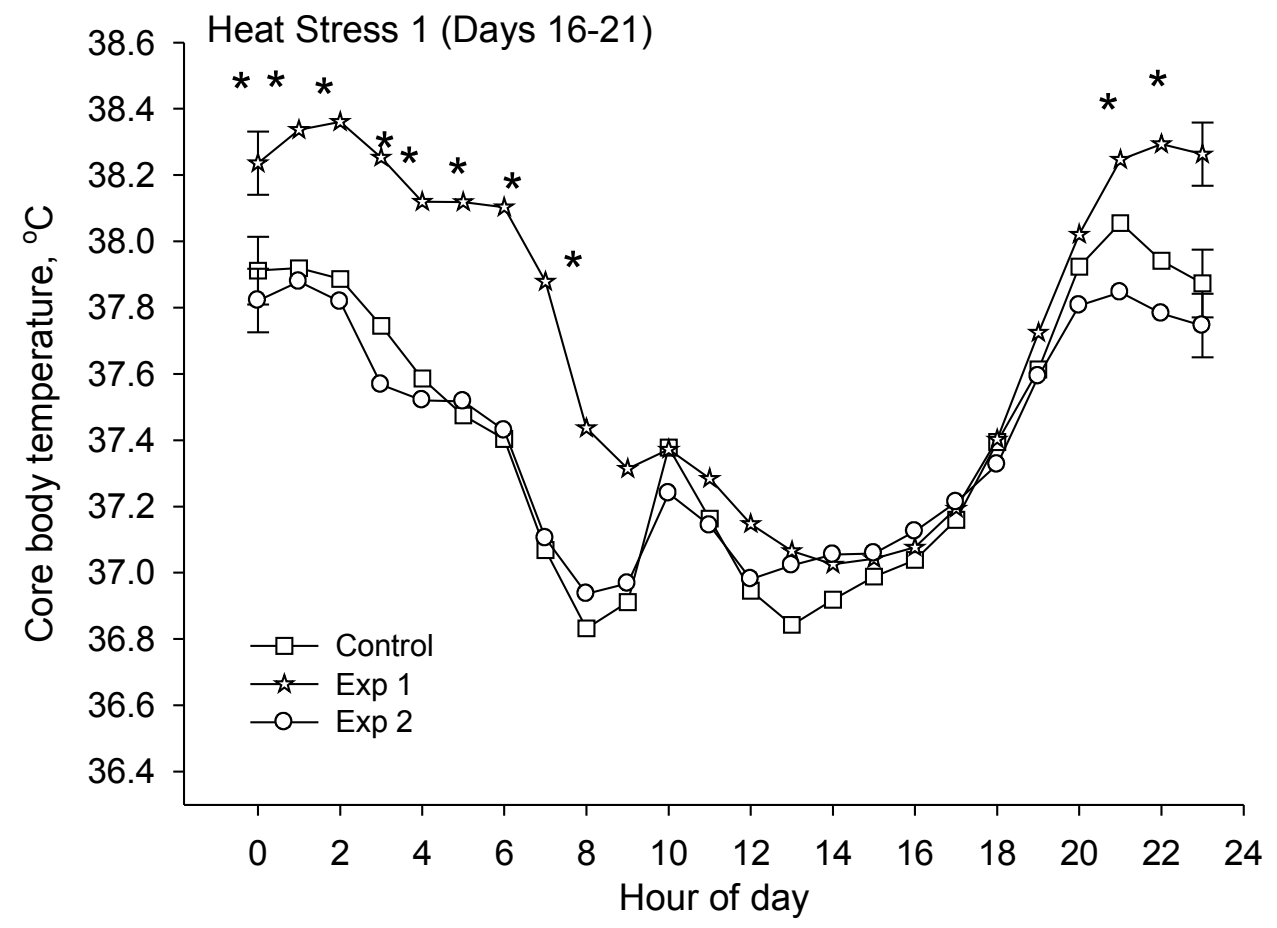

Figure 1.9. Hourly core body temperature averaged by group and averaged for days 16 to 21 . An asterisk $(*)$ indicates a significant difference from previous hour of day Control, Exp. 1, and Exp. 2 groups. The vertical lines above and below the first and last points indicate \pm SE. 


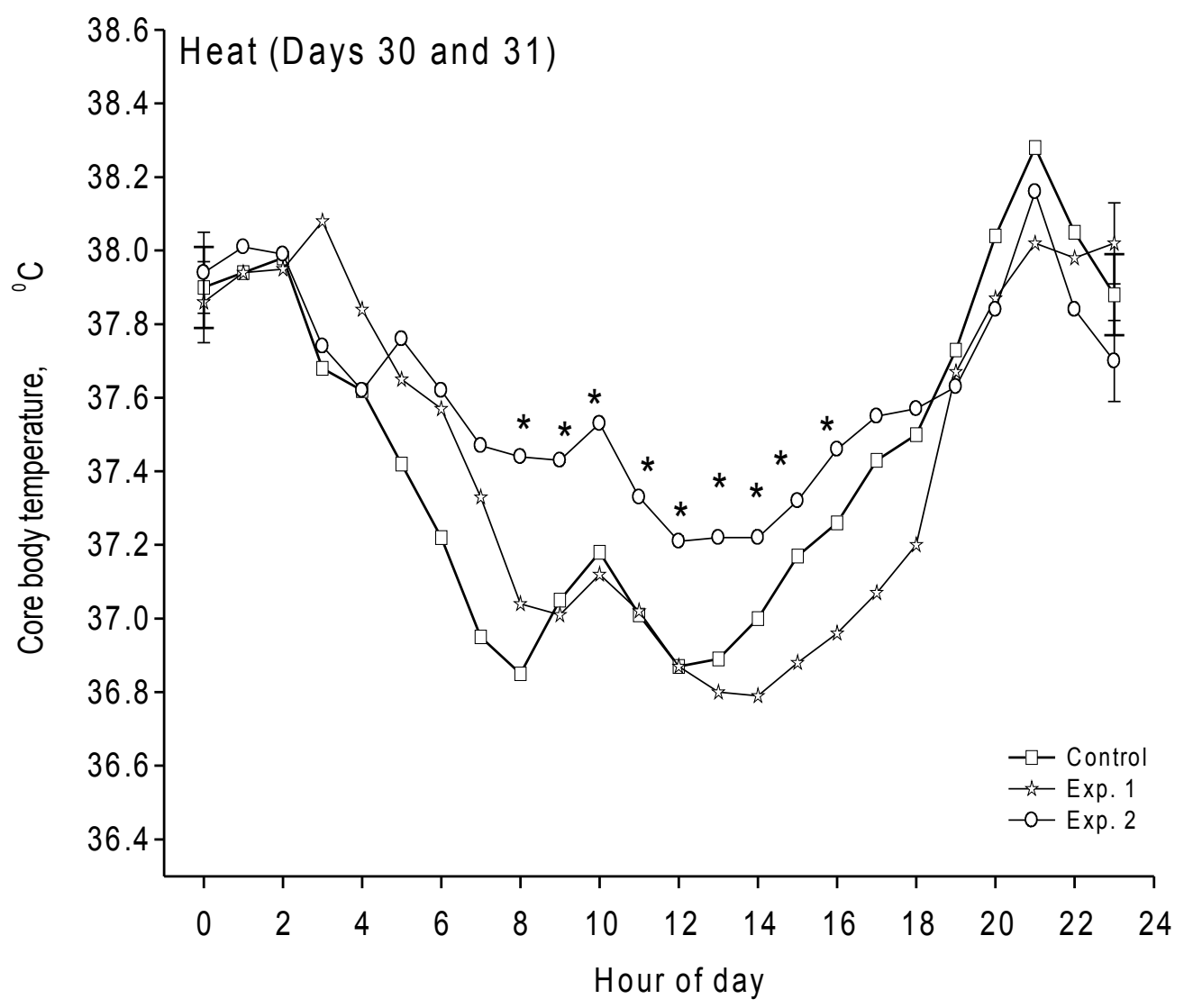

Figure 1.10. Hourly core body temperature averaged by group and averaged for days 30 and 31 . An asterisk $(*)$ indicates a significant difference from previous hour of day Control, Exp. 1, and Exp. 2 groups. The vertical lines above and below the first and last points indicate \pm SE. 


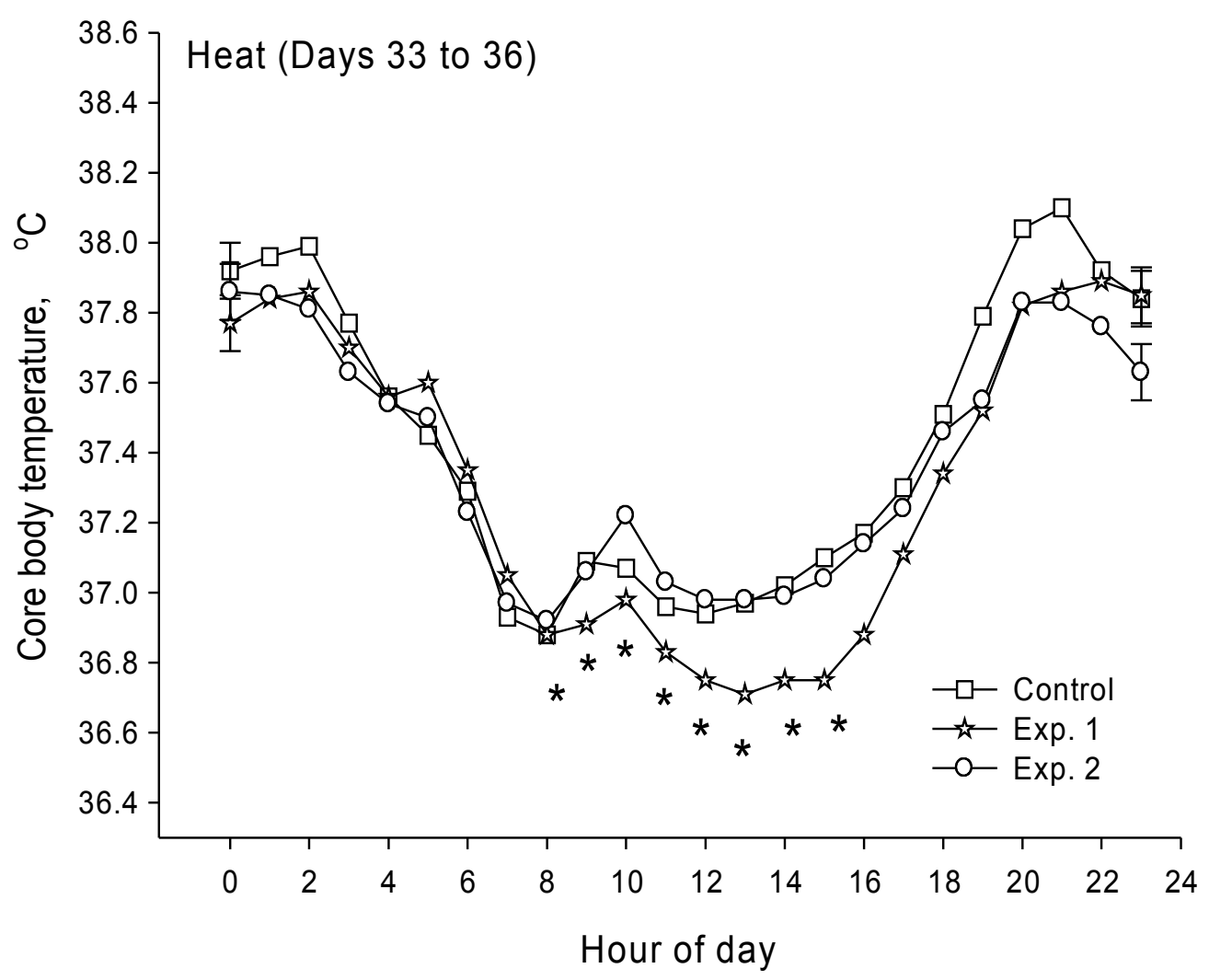

Figure 1.11. Hourly core body temperature averaged by group and averaged for days 33 to 36 for Control, Exp. 1, and Exp. 2 groups. An asterisk (*) indicates a significant difference from previous hour of day for Control, Exp. 1, and Exp. 2 group The vertical lines above and below the first and last points indicate $\pm \mathrm{SE}$. 


\section{CHAPTER II}

\section{EFFECT OF HEAT STRESS AND ERGOPEPTINE ALKALOIDS ON THE IMMUNE SYSTEM OF RATS}

\section{Abstract}

There is very limited information on the effects of heat stress and ergopeptine alkaloids on the immune system. Therefore we looked at a broad immune response in the rat model used to study fescue toxicosis. Rats were implanted with intraperitoneal telemetric transmitters to measure core body temperature (Tc) and activity. They were fed diets containing ergopeptine alkaloids $(\mathrm{E}+)$, minus alkaloids (E-), or pair-fed (PF to $\mathrm{E}+$ ). Ergovaline concentration of alkaloids (E+; 32 ug ergovaline/kg BW/d) was measured using HPLC. Groups were further divided into temperature treatments of thermoneutrality $\left(\mathrm{TN} ; 21^{\circ} \mathrm{C}\right)$ or heat stress $\left(\mathrm{HS} ; 33^{\circ} \mathrm{C}\right)$. All rats received diet treatments at TN for 1 week followed by 3 days of constant HS or TN treatment. On the fourth day, half of the rats in each treatment group received an intraperitoneal lipopolysaccharide (LPS) challenge $(100 \mu \mathrm{g} / \mathrm{kg}$ body weight) and the other half were injected with sterile saline. Feed intake and body weight were reduced by the alkaloids, heat stress, and the combination of both stressors. Likewise, the combination of both stressors increased Tc above other heat-stressed groups. Blood samples were collected at trial end and analyzed by flow cytometry and Quantikine ELISA kits. The combination of stressors diminished B cells by one-half $(\mathrm{P}<0.05)$, while neither heat stress nor endophyte had any effect. Both TNF $\alpha$ and IL1 $\beta$ levels of LPS challenged rats were higher $(\mathrm{P}<0.05)$ than control rats. The levels in the $\mathrm{E}+\mathrm{HS}$ group were highest $(\mathrm{P}<0.05)$ among treatment groups. In summary, combination of 
alkaloid intake and heat stress significantly affected the levels of circulating B cells, and IL $\beta$, with more modest $(\mathrm{P}<0.05)$ changes in $\mathrm{T}$ cells. Caloric restriction (PF) increased NK cells, regardless of environmental conditions. The combined effect of heat stress and intake of ergopeptine alkaloids associated with fescue toxicosis impact the immune system during short-term heat stress. If these are observed in cattle model they may be susceptible to infection during periods of initial heat stress.

\section{Introduction}

Tall fescue (Lolium arundinacea) is widely used in the Midwest as pasture grass. It is considerably resistant to insect attacks and thrives well under marginal, acidic, and poorly drained soil. These advantages are related to its symbiotic relationship with the fungus Lolium arundinaceum (Malinowski et al., 2005). However, this relationship is the primary cause of fescue toxicosis in animals, which causes annual losses of over 600 million dollars in the United States (Hoveland et al., 1993). The toxins cause a wide range of clinically non-specific symptoms like reduced FI, body weight gain, and activity in animals (Stickland et al., 1995). Other syndromes are fescue foot and summer slump. A very characteristic feature of fescue toxicosis is a marked reduction in serum prolactin level (Mizinga et al., 1993; Thompson et al., 2001). This, could lead to agalactia and a compromised immune system.

Effects of fescue toxicosis in the immune system have been variable. Studies using cattle and mouse models have shown that fescue toxicosis lowers white blood cell count, mononuclear phagocytic activity, and serum prolactin levels. In addition, it alters the lymphocyte neutrophil ratio, impacts $\mathrm{T}$ lymphocytes, and affects genes related to immune system (Saker et al., 1997; Filipov et al., 1999a; Dew et al.,1990; Settivari et al., 2006). Similarly, intake of ergot alkaloids decreases eosinophil count and $\gamma$-globulin levels in cattle (Oliver et al., 2000), as well as increases clot formation 
and release of acute phase proteins (Oliver et al., 2000). A long-term study in mice at thermoneutrality reported an increase in spleen T-suppressor cells of rats fed endophyte infected diet (Dew et al., 1990). This could suppress the cell-mediated response and make rats susceptible to extracellular and intracellular pathogens. Few studies have attempted to differentiate the effects ergopeptine alkaloids of immune system under thermoneutral and heat stress conditions. Likewise, studies to identify effects of fescue toxicosis, heat stress, caloric restriction and their combination on the immune system have not been performed. In our study, we hypothesized that the combined effect of heat stress and fescue toxicosis could impact the immune system more than either acting alone. We also hypothesized that short-term heat stress and fescue toxicosis may not allow the animal enough time to adapt to these stressors. 


\section{Materials and Methods}

\section{Animals and treatment diets}

Male Spargue Dawley rats $(\mathrm{n}=72 ; 350$ to $400 \mathrm{~g}$ BW) were implanted intraperitoneally, with telemetric transponders (Respironics, Bend, OR) to record core body temperature (Tc) and activity (Appendix 1). They were then were allowed a post operative recovery period of one week, and placed in to two chambers with similar conditions and ambient temperature. Dataloggers (HOBO Boxcar Prodata; Onset Computer Corp., Bourne, MA) were placed in each chamber to record ambient temperature and percent relative humidity throughout the study. Animals were maintained in 12:12 day:light cycles, and percent relative humidity was maintained at $50 \pm 5 \%$, which was similar to previous studies in our lab (Spiers et al., 2005, Settivari et al., 2006). Feed and water were provided ad libitum throughtout the study period. Rats were fed either uninfected tall fescue seeds (E-) or infected tall fescue seed (E+; $32 \mu \mathrm{g} \mathrm{EV/kg} \mathrm{BW/d).} \mathrm{These} \mathrm{levels} \mathrm{were} \mathrm{estimated} \mathrm{by} \mathrm{standard} \mathrm{protocol}$ using HPLC (Rottinghaus et al 1993). Composition of diet was similar to other studies in our lab (Spiers et al., 2005). All diets were stored at $4^{\circ} \mathrm{C}$ before feeding rats. FI (FI) and body weight were measured every day at 0900. Pair-fed (PF) diet was calculated by dividing the average FI of E+ animals by the average body weight on that particular day. The net value was multiplied by the body weight of each PF animal to calculate the amount of feed, for that day

Rats were randomly divided into 6 treatment groups: E+ diet fed at thermoneutrality $(\mathrm{E}+\mathrm{TN}), \mathrm{E}-\operatorname{diet}$ fed at TN $(\mathrm{E}-\mathrm{TN}), \mathrm{E}-\operatorname{diet}$ pair-fed to E+ TN at TN (PF to $\mathrm{E}+\mathrm{TN}), \mathrm{E}+$ diet fed 7 days at TN followed by 3 days at HS, (E+HS), E- diet fed 7 days TN followed by 3 days at HS (E-HS), E- diet pair-fed to E+ HS at HS.(PF to E+ HS). Thermoneutral and heat stress groups were housed in separate chambers. 


\section{Experimental design.}

Once FI and Tc were stabilized, baseline values for these parameters were collected for all animals in both rooms (HS and TN) for a period of five days. Following this period, rats in both chambers received their respective treatments (i.e., $\mathrm{E}+, \mathrm{E}-$, and $\mathrm{PF}$ to $\mathrm{E}+$ ) at $\mathrm{TN}$, for a period of one week. Following this period, ambient temperature of one of the rooms was increased to a constant temperature of $33^{\circ} \mathrm{C}$ for rest of the study and the other chamber was continuously maintained at $21^{\circ} \mathrm{C}$ (Figure. 2). On the fourth day of this period at 0830, half of the rats from each treatment groups $(\mathrm{n}=6)$ received an intraperitoneal LPS injection $(100 \mu \mathrm{g} / \mathrm{kg} \mathrm{BW})$ and other half $(n=6)$ received a control intraperitoneal injection of $2 \mathrm{mls}$ sterile saline. After 2 hours, rats were anesthetized using xylazine $(13 \mathrm{mg} / \mathrm{ml})$ and ketamine $(87 \mathrm{mg} / \mathrm{ml})$, at dose rate of $0.1 \mathrm{mg} / \mathrm{kg} \mathrm{BW}$. Blood samples were collected using intracardiac puncture.

\section{Sample preparation}

Whole blood was collected in heparinized tubes and processed using the ficollhypaque centrifugation method for isolation of lymphocytes (Appendix 2). All analyses were done at the University of Missouri hospital, flow cytometry core facility). Samples were counted by flow cytometery in the cell and immunobiology core facility, at the University of Missouri hospital using BD FASC calibur instrument. Serum was separated from whole blood for cytokine assays, using Quantikine IL-1 assays and Quantikine TNF $\alpha$ (R \& D systems).

\section{Statistical analysis}


All data were analyzed using Analysis of Variance ANOVA (JMP® 8; SAS Institute, Inc., Cary, NC). Repeated measures ANOVA of SAS (SAS Institute,,Inc, Cary, NC) was used to ascertain time-related changes in FI, body weight, core body temperature, motor activity, and levels of lymphocytes and cytokines. Standard least square model fit (JMP®, SAS Institute Inc, Cary, NC) was used for analysis of all parameters. Significance was reported based on the Tukey-Kramer multiple comparison test (Steele and Torrie, 1981). A P value of less than 0.05 was reported as significant. A linear plot analysis was performed using the mean weight all rats in a treatment for a particular day.

\section{Results}

\section{Feed intake}

\section{Pretreatment}

During pretreatment there were no significant difference in FI among treatment groups (Figure. 2.1). Likewise, there was no endophyte $(\mathrm{P}>0.20)$ or endophyte by day effects $(\mathrm{P}>0.72)$.

\section{Diet Treatment}

Feed inatke of E- rats was unaffected during the initial diet treatment at TN. Rats fed the $\mathrm{E}+$ diet showed a significant reduction in FI on day one of diet treatment $(\mathrm{P}<$ 0.0001). During diet treatment (Days 1 to 7$)$, both day $(\mathrm{P}<0.0001)$ and day by diet $(\mathrm{P}$ $<0.0001)$ effects were observed. The FI of $\mathrm{E}+$ and $\mathrm{PF}$ TO E + rats stayed below $(\mathrm{P}<$ 0.05) FI of E- rats during days 1 to 5 of diet treatment (Figure. 2.1). Partial recovery in FI was observed on Days 4 to $7(\mathrm{P}>0.05)$. However, the FI of $\mathrm{E}+$ rats never reached that of E- rats (Figure. 2.2). 


\section{Heat Stress and diet treatment}

The FI of all treatment groups at TN was lower than HS groups $(\mathrm{P}<0.05$; Figure. 2.3). However, there was no significant difference among treatment groups at $\mathrm{TN}$ from days 8 to 10 . The $\mathrm{E}+\mathrm{TN}$ and $\mathrm{PF}$ TO $\mathrm{E}+\mathrm{TN}$ rats consumed more $(\mathrm{P}<0.05)$ feed compared to E+ HS and PF TO E+ HS rats. The FI of E- TN was consistently higher than E- HS from Days 8 to 10 (Figure. 2.3).

Rats reduced their FI in response to endophyte, HS and a combination of both (P $<0.05$, Figure. 2.4). The E+ HS rats reduced FI on the first HS day by $69 \%$ from Day 7 of diet treatment compared to E- HS rats which reduced their FI by $62 \%$ (Day 8). The PF TO E+ group followed the same pattern of E+ HS rats as expected, but the effects were seen the following day, as FI was calculated based on FI of E+ HS group. Both diet and temperature effects were observed $(\mathrm{P}<0.0001)$ during Days 8 to 10 .

Rats in the short-term HS studydid not show any signs of FI recovery. Comparisons between E- HS and E+HS groups showed that E-HS rats consumed more compared to $\mathrm{E}+\mathrm{HS}$ rats $(\mathrm{P}<0.05)$. In contrast, rats at $\mathrm{TN}$ showed partial FI recovery. The FI of E+ TN rats was highest on Days 9 and 10, with PF TO E+ TN rats following this a day later.

\section{Body weight}

\section{Pretreatment period}

There was no endophyte $(\mathrm{P}>0.16)$ or endophyte by day effects $(\mathrm{P}>0.16)$ on body weight during pretreatment period. However, a significant day effect $(\mathrm{P}<$ 
0.0001) was observed in average daily body weight gain (Figure. 2.4). This was expected since the rats were still within the standard growth phase for their age group.

\section{Diet treatment}

The E- treatment groups continued to grow consistently at the normal rate during the diet treatment phase at TN. There was, however, a decrease in body weight in response to the $\mathrm{E}+$ diet, with the $\mathrm{PF}$ to $\mathrm{E}+$ group following a similar pattern a day later (Figure. 2.5). Although there was no overall endophyte effect, there was a day effect and an endophyte by day effect $(\mathrm{P}<0.0001)$. The linear slope values showed that ETN rats increased $(\mathrm{P}<0.05)$ their body weight $(+2.7 \mathrm{~g} / \mathrm{d})$ significantly above $\mathrm{E}+\mathrm{TN}$ and PF TO E+TN rats, which lost $-2.9 \mathrm{~g} / \mathrm{d}$ and $-1.9 \mathrm{~g} / \mathrm{d}$, respectively.

\section{Heat Stress and diet treatment}

The E- TN rats continued to increase $(\mathrm{P}<0.05)$ their body weight until the end of study. There was gradual increase $(\mathrm{P}<0.05)$ in the body weight of $\mathrm{E}+\mathrm{TN}$ rats, and $\mathrm{PF}$ TO E+ animals at TN followed a similar pattern. On Days 8 to 10 , body weight of $\mathrm{E}+\mathrm{TN}$ rats was not significantly different from E-TN rats.

The body weight of rats dropped $(\mathrm{P}<0.05)$ in response to $\mathrm{HS}\left(33^{\circ} \mathrm{C}\right)$. As stated before, the effects of weight gain are observed after the drop in FI and paired fed effects are seen a day after E+ rats. Considering this, the body weight of all treatment groups during HS at Days 9 and 10 was lower $(\mathrm{P}<0.05)$ than rats maintained at TN (Figure. 2.6). Thermal, thermal by day, and thermal by day by endophyte effects were observed $(\mathrm{P}<0.0001)$. At Day 10 , the body weight of $\mathrm{E}+\mathrm{HS}(\mathrm{P}<0.05)$ was lower than all other treatment groups in the study. This indicates that E+ HS could have a greater impact on body weight gain compared to either stressors acting alone. 
A linear regression plot showed that the E-TN, E+TN, and PF TO E+TN rats gained 5.6, 0.88 and $4.83 \mathrm{~g} / \mathrm{d}$, respectively, from Days 8 to 10 . The body weight of rats at TN was significantly $(\mathrm{P}<0.05)$ greater than the body weight of all HS rats. A significant reduction in body weight of all HS groups was observed. The E+HS, EHS, and PF TO E+HS rats lost 20.80, 17.16 , and $11.80 \mathrm{~g} / \mathrm{d}$, respectively.

The rats at $\mathrm{TN}$ adapted to the toxin, with the $\mathrm{E}+\mathrm{TN}$ group gaining weight consistantly during the last three days of study but not reaching E-TN level. The PF $\mathrm{TO} \mathrm{E}+\mathrm{TN}$ rats followed a similar pattern. Rats fed $\mathrm{E}+$ at $33^{\circ} \mathrm{C}$ did not show any signs of adaptation until Day 10 of study. Also, E-HS rats did not exhibit a positive growth rate. However, the body weight of the E-HS group was higher than the E+HS group

\section{Core body temperature}

\section{Pretreament period}

The average Tc was not significantly different among treatment groups. Diet $(\mathrm{P}>$ $0.16)$ and diet by day $(\mathrm{P}>0.72)$ effects were not observed. The average Tc varied from 37.3 to $37.5^{\circ} \mathrm{C}$ among treatment groups (Figure. 2.7).

\section{Diet treatment}

Rats showed significant day $(\mathrm{P}<0.0001)$ and endophyte by day $(\mathrm{P}<0.0001)$ effects in response to diet treatment. The E- groups did not show any significant variation in average daily $\mathrm{Tc}$ and it ranged from 37.4 to $37.5^{\circ} \mathrm{C}$ (i.e., similar to pretreatment levels). Average daily Tc of rats was lower in $\mathrm{E}+$ groups in response to the endophyte. The notable decrease ( $P<0.05$, Figure. 2.8) in Tc was on days 4 to 7 . On Days 6 and 7, there was a $0.17^{\circ} \mathrm{C}$ decline from day one of diet treatment. During 
diet treatment, PF TO E+ rats also had lower Tc compared to E- rats. The Tc of these rats was lower on Days 5 to 7 compared to first two days of diet treatment. However, these effects were not significant $(\mathrm{P}>0.30)$.

\section{Heat stress and diet treatment}

Significant thermal, day, and thermal by day effects $(\mathrm{P}<0.0001)$ were observed during the thermal treatment period. Rats at TN did not show any significant changes in their Tc $(\mathrm{P}>0.58)$. The E-TN rats had slightly higher Tc than E+TN and PF TO E+TN. There was, however, an increase $(\mathrm{P}<0.05)$ in daily Tc of E- HS, E+ HS and PF TO E+ HS (Figure. 2.7). On the first day (Day 8) of HS, E+ HS had the largest increase $(\mathrm{P}<0.05)$ of $0.9^{\circ} \mathrm{C}$ above day 7 level at $\mathrm{TN}$. The E- HS group increased $(\mathrm{P}<$ 0.05) $0.5^{\circ} \mathrm{C}$ above Day 7 level on the first HS day (Figure. 2.8). On day 8, E+ HS, EHS, and PF TO E+ were not significantly different from one another, but were significantly $(\mathrm{P}<0.05)$ higher than TN groups. However, on Day $10 \mathrm{E}+\mathrm{HS}$ was significantly higher than E-HS and PF TO E+ TN groups (Figure. 2.9). By Day 10, $\mathrm{E}+\mathrm{HS}$ had increased $(\mathrm{P}<0.05)$ another $0.9^{\circ} \mathrm{C}$ from first $\mathrm{HS}$ day.

Core body temperature of rats maintained at $\mathrm{TN}$ did not return to pretreatment levels. However, they stabilized on Day 8 of study and did not drop any further. On day $10, \mathrm{E}+\mathrm{TN}$ Tc was $0.32{ }^{\circ} \mathrm{C}$ lower than its value on day one. Similarly, PF TO E+ also had a lower Tc and it was $0.14^{\circ} \mathrm{C}$ lower than on day one. This shows that the reduction in Tc associated with the intake of ergopeptide alkaloids might be indirectly attributed to the reduction in caloric intake associated with fescue toxicosis. Core body temperature in the HS groups continued to increase until day 10 and did not show signs of adaptation. The Tc of E+HS rats on Day 10 was $0.52{ }^{\circ} \mathrm{C}$ above E- HS level, which suggests that the ability of E+HS rats to dissipate body heat is 
compromised more compared to E-HS rats. The PF TO E+ HS rats had a Tc that was similar to E- HS rats.

Circadian changes in core body temperature

\section{Pretreatment}

Significant hour and thermal by hour effects $(\mathrm{P}<0.0001)$ were observed in Tc patterns. During pretreatment period, the highest Tc was observed at 2100 to 0200 when the nocturnal rats are more active. The lowest Tc recorded was around 08000900 once the lights turn on in the chamber. Core body temperature decreased $(\mathrm{P}<$ 0.05 ) from 0600 to 0700 before lights were turned on and started to increase from 1700 to 1800 before lights were turned off. But a greater increase $(\mathrm{P}<0.05)$ was observed after 2100. A spike in Tc was seen in response to daily feeding time.

\section{Diet treatment}

Diet treatment showed significant hour, endophyte by hour, and thermal by hour effects $(\mathrm{P}<0.0001)$. Both the $\mathrm{E}+$ and $\mathrm{PF}$ TO E+ groups had a lower $(\mathrm{P}<0.05) \mathrm{Tc}$ from 0700 to 1000 compared to 2100 to 0200 during this period (Figure. 2.10). Likewise, the Tc for these groups stayed lower $(\mathrm{P}<0.05)$ throughout the day when the lights were on. However, Tc for these groups increased after the lights were turned off and then did not differ from E- groups. This suggests that TN hypothermia in $\mathrm{E}+$ rats might be best documented during day when lights are turned on. A daily increase in Tc was documented in response to feeding, and PF rats had the greatest increase in Tc as they consume feed immediately. 


\section{Heat stress and diet treatment}

Significant thermal, hour, and thermal by hour effects $(\mathrm{P}<0.0001)$ were observed. Heat stress increased $(\mathrm{P}<0.05) \mathrm{Tc}$ of rats in all HS groups. However, they followed the same circadian pattern as TN rats (Figure. 2.11). The only difference was the absence of a pronounced increase after feeding in E-HS and E+HS rats. Greater $(\mathrm{P}<0.05)$ differences in $\mathrm{Tc}$ between $\mathrm{HS}$ and rats at $\mathrm{TN}$ were observed from 2100 to 0700 when rats were active (Figure. 2.11). Interestingly, E+ HS had a significantly $(\mathrm{P}<0.05)$ higher Tc compared to E-HS and PF TO E+HS from 0900 to 0200. This suggests that difference between E+HS and E-HS is best noted at night when Tc increases.

\section{General gross motor activity}

\section{Pretreatment}

During pretreatment period, gross motor activity did not show endophyte $(\mathrm{P}>$ $0.32)$ or endophyte by day effects $(\mathrm{P}>0.85)$.

\section{Diet treatment}

During the diet treatment period, endophyte $(\mathrm{P}>0.01)$ and endophyte by day $(\mathrm{P}$ $>0.92$ ) effects were absent. Treatment groups did not differ significantly, however E+ groups had a trend towards reduced acitivity (Figure. 2.12).

\section{Thermal treatment and diet treatment}

Rats showed both day $(\mathrm{P}<0.0001)$ and thermal by day effects $(\mathrm{P}<0.001)$ during days 8 to 10 . Activity of rats maintained in TN did not differ significantly among 
treatment groups $(\mathrm{P}<0.09)$. During thermal treatment all HS rats had a slightly higher activity compared to rats at TN on the first day (Day 8) of HS, and then reduced their gross motor activity. The E- HS group had a higher activity $(\mathrm{P}<0.05)$ compared to all other treatment groups on Days 8 and 9, and then reduced their activity on day 10 (Figure. 2.12). On Day10, E+ HS rats had the lowest activity followed by $\mathrm{E}+\mathrm{TN}$ rats, which suggest that activity could be a measure of sickness behaviour.

\section{Circadian changes in activity}

\section{Pretreatment}

A set pattern of daily activity was observed during the entire study. Circadian rhythm of activity was similar to Tc (Figure. 2.14). The large decline in activity was seen from 0600 to $0700(\mathrm{P}<0.05)$ before lights were turned on, and an increase in activity was seen from 1700-1800 before lights were turned off. A greater increase in activity was observed from 1800-1900, and the lowest activity was observed at 0800 . A significant increase from previous hour was observed in all treatment groups at 1000, associated with the daily feeding time. A diet effect $(\mathrm{P}>0.57)$ was not observed.

\section{Diet treatment}

During this period, the circadian rhythm of activity was similar to pretreatment levels and did not change significantly. Hour, endophyte by hour, and thermal by hour effects $(\mathrm{P}<0.0001)$ were observed (Figure. 2.13). The $\mathrm{E}+$ treatment did not have a significant effect on the circadian pattern of activity $(\mathrm{P}>0.35)$. 


\section{Heat stress and diet treatment}

There were significant thermal, hour, endophyte by hour, and thermal by hour effects $(\mathrm{P}<0.0001)$ during HS (Figure. 2.14). The rats maintained at TN followed a similar pattern observed during diet treatment. The E+ HS rats had lower activity (P $<0.05)$ compared to E-TN from 1000 to 1100 . This is attributed to the reduced FI possibly due to reduced interest in eating with two stressors (i.e., HS and E+).

\section{Lymphocytes}

Lymphocytes are white blood cells which can be broadly classified into NK cells, T-lymphocytes, and B lymphocytes, with CD4+ (helper T) and CD8+ (cytotoxic T) cells being subsets of T-lymphocytes (Abbas et al., 2007). Using flow cytometry, the percentage of each cell in the peripheral blood of rats was quantified.

\section{Natural killer cells}

The NK cells play an important role in innate immune response and act against intracellular virus and tumor cells. The E-TN did not differ significantly from E+TN group. However, the E-TN and E+TN groups were lower $(\mathrm{P}<0.05)$ than the $\mathrm{E}+\mathrm{HS}$ group. The E+HS group percentage was highest among all treatment groups (Figure. 2.15). Pair-feeding increased NK cell percentages irrespective of environmental conditions. Heat stress only $(\mathrm{P}>0.06)$ and endophyte $(\mathrm{P}>0.58)$ did not have any significant effects on NK cells.

\section{B-lymphocytes}


The B-lymphocyte percentage of E- TN, E+TN, and PF TO E+ TN groups did not differ significantly. The E+ HS percentage was the lowest and significantly $(\mathrm{P}<$ 0.05) below E- HS, PF TO E+ HS, and E+TN groups (Figure. 2.16). Endophyte (P > 0.66) and heat stress $(\mathrm{P}>0.67)$ individually did not impact $\mathrm{B}$ cell percentages.

\section{T-lymphocytes}

Total T-lymphocyte level of E- TN, PF TO E+TN, and E+TN groups did not differ significantly. The E+TN was slightly loweR $(\mathrm{P}>0.15)$ than other groups at TN. T-lymphocyte percentage of the HS groups did not differ significantly (Figure. 2.17). However, the E+ HS group was higher than other HS groups and significantly $(\mathrm{P}<0.05)$ greater than the $\mathrm{E}+\mathrm{TN}$ group. There were no thermal or diet effects.

\section{CD4+ Cells}

The CD4+ cells are a subset of T-lymphocytes. These cells did not differ significantly among treatment groups. Diet $(\mathrm{P}>0.22)$ and thermal effects $(\mathrm{P}>0.83)$ were not observed.

\section{CD8+ Cells}

The CD8+ cells are a subset of T-lymphocytes. These cells did not differ significantly among treatment groups. Diet $(\mathrm{P}>0.75)$ and thermal effects $(\mathrm{P}>0.83)$ were not observed.

\section{Core body temperature and activity in response to LPS}

\section{LPS at thermoneutrality}


Core body temperatures of all LPS-challenged rats did not differ significantly from LPS control rats. However, both hour $(\mathrm{P}<0.0007)$ and thermal effects $(\mathrm{P}<$ $0.0001)$ were observed. No LPS $(\mathrm{P}>0.70)$ and LPS by hour $(\mathrm{P}>0.81)$ effects were seen. Core Body temperature of E- TN LPS rats showed a marginal $(\mathrm{P}>0.036)$ increase from 0800 to 0900 , but dropped by 1000 (Figure. 2.18). The Tc of E-TN LPS- rats decreased $0.22^{\circ} \mathrm{C}$ at 1000 , following a natural circadian pattern. Likewise, the Tc of E- TN LPS+ rats dropped $0.36^{\circ} \mathrm{C}$ at 1000 , (i.e., 2 hours after initiation of LPS challenge). The largest Tc reduction occurred in the E+TN LPS+ rats with a $0.76^{\circ} \mathrm{C}$ decrease from its preinjection level at 0800 . In contrast, Tc of PF TO E $+\mathrm{TN}$ LPS + rats showed a non-significant increase at 1000. Overall, there was a trend towards hypothermia with LPS injection at TN.

Activity followed the same trend as Tc, with all rats exhibiting lower activity at 1000. In contrast, LPS-challenged rats exhibited a greater but non-significant decrease in activity compared to LPS control rats. There were both hour and thermal effects $(\mathrm{P}<0.0001)$. However, there were no LPS $(\mathrm{P}>0.54)$ and LPS by hour effects $(\mathrm{P}>0.41)$

\section{LPS during heat stress}

For the HS test, there was the same pattern of an initial spike from 0800 to 0900 for all treatment groups. There was both an hour $(\mathrm{P}<0.0007)$ and a thermal effect $(\mathrm{P}$ $<0.0001)$. However, LPS $(\mathrm{P}>0.70)$ and LPS by hour $(\mathrm{P}>0.81)$ effects were not seen. Similar to the TN test, LPS control rats decreased Tc from 0900 to 1000 . Both E+ HS LPS+ and E-HS LPS+ groups increased Tc slightly $(\mathrm{P}>0.81)$ from 0900-1000 (Figure. 2.19). This suggests that there is a trend towards hyperthermia with LPS in heat stressed rats. 
There was a trend for an increase in general activity $(\mathrm{P}>0.42)$, followed by a decrease that was observed for the HS conditions. The E+HS LPS+ rats had the lowest activity at $1000(\mathrm{P}>0.15)$. All other rats also had a lower activity at 1000 , following their natural circadian pattern.

\section{Cytokine response to LPS administration}

\section{IL1及}

All treatment groups at TN and under HS conditions responded with an increase in circulating levels of IL $1 \beta$, when $100 \mu \mathrm{g} / \mathrm{kg}$ BW of LPS was administered intraperitoneally. Two hours after LPS challenge all treatment groups that had received LPS had higher levels of circulating IL1 $\beta,(\mathrm{P}<0.05)$ compared to LPS control groups (Figure. 2.20). A thermal effect $(\mathrm{P}<0.03)$ showed that $\mathrm{E}-\mathrm{TN}$ and $\mathrm{PF}$ TO E+TN had lower circulating levels of IL1 $\beta$, compared to the respective groups in heat stress. The E- TN, PF TO E+ TN and E+TN levels did not differ significantly. The E+ HS LPS + group had the highest $(\mathrm{P}<0.05)$ IL1 $\beta$ level after two hours of challenge. The IL1 $\beta$ concentration was lowest in PF E+ HS LPS+ rats, where it was significantly $(\mathrm{P}<0.05)$ lower than PF TO E+ TN LPS + and E+HS LPS + rats. There was no significant difference among LPS control groups based on diet or thermal treatment.

\section{TNFa}

All LPS challenged groups responded with a higher circulating level of TNF $\alpha$ compared to LPS control groups (Figure. 2.21). The E- TN, PF TO E+ TN, and E+TN levels did not differ significantly. Both $\operatorname{diet}(\mathrm{P}<0.02)$ and thermal effects $(\mathrm{P}<0.02)$ were observed. The E- HS rats had the highest $(\mathrm{P}<0.05)$ circulating levels of TNF $\alpha$. 
Interestingly, $\mathrm{TNF} \alpha$ levels of $\mathrm{E}+\mathrm{HS}$ was lowest $(\mathrm{P}<0.05)$ among treatment groups. The PF to E+ HS rats had slightly lower levels than the same group maintained at TN. The LPS control rats had levels of TNF $\alpha$ that were undetectable.

\section{Discussion}

\section{Feed intake}

In this experiment, FI was reduced by $50 \%$ on the first day of diet treatment at thermoneutrality. Other studies have also reported similar decreases in FI in response to endophyte infected fescue (Roberts et al., 2002; Spiers et al., 2005; Al-Tamimi et al., 2007; Settivari et al., 2008) FI showed partial recovery on day 4, and this is consistent with past studies (Robert et al., 2002; Spiers et al., 2005). Likewise, FI was reduced in response to heat stress and endophyte, and a greater decrease was documented with the combination of both. These results are consistent with previous reports (Spiers et al., 2005; Al-Tamimi et al., 2007). Rats that received ergopeptine alakaloids during heat stress reduced their FI by $69 \%$ compared to rats only exposed to heat stress that reduced their FI by $62 \%$ on the first day of heat stress. These findings are greater than the values from previous reports. A higher magnitude of reduction in FI is attributed to the higher thermal stress $\left(33^{\circ} \mathrm{C}\right)$ used in the present study, compared to past studies. Contrary to previous reports by Settivari et al (2006), no adaptation to FI was observed until Day 10. This could also be related to the effects of higher heat stress. Likewise, Spiers et al. (2005) did not observe any adaptation of FI to short-term heat stress. However, adaptation of FI observed on days 9 and 10 at thermoneutrality was in agreement with previous reports from our laboratory (Settivari et al., 2006). There are several theories proposed stating various reasons for the reduction in FI associated with fescue toxicosis. Strickland et al. 
(1993) related effects of fescue toxin and dopamine activation. Dopamine activation may cause satiety leading to decreased FI. Other studies have shown that alterations serotonin and dopamine could lead to satiety and decreased FI in rats and cattle model used to study fescue toxicosis (Oliver et al., 1997; 2005; Larson et al., 1999). AlTamimi et al. (2007) stated that fescue impacts 5-HT receptors causing reduction in FI. There is consensus that hunger and FI are controlled by different factors. Therefore, fescue toxicosis could also act through more than one mechanism. Reduced FI is seen as a protective mechanism to avoid postprandial metabolic heat production. This improves the animal's ability to cope with the increased body temperature.

\section{Body weight}

Body weight gain was reduced in response to change in FI in the present study. Significant reductions in body weight were observed immediately after the rats were placed on the endophyte-infected diet and following the reduction in FI. They continued to lose body weight until day five. The first increase in body weight was observed on day six. Previously a $63 \%$ decrease in average daily gain was observed when rats were fed an endophyte-infected diet compared to those fed an uninfected diet at thermoneutrality (Neal and Schmidt., 1985). Similar, decrease of 22 to $79 \%$ was also observed in cattle, when fed with endophyte infected diet at thermoneutrality (Schmidt et al., 1982). The reduction in body weight gain in thermoneutrality is similar to past studies in our lab were rats decrease their body weight until days four to six in thermoneutrality and then begin to stabilize and gain body weight (Spiers et a., 2005; Settivari et al., 2006). Roberts et al. (2002) reported that rats stabilized their body weight at day four and then began to increase their body weight when fed E+ 
diet in thermoneutral conditions. Such effects were not documented in the control rats and they gained weight continuously. The pattern of decreased body weight gain paralled FI, therefore we propose that reduced FI contributes to decreased FI.

Heat stress decreased average daily gain in E-, E+, and PF TO E+ treatment groups. The impact of heat stress on body weight gain was greater than the effects of endophyte only. A decrease in body weight gain in response to heat stress in well established in the rat model and this may be an effort to lower metabolism and decrease metabolic heat production (Johnson and Cabanac, 1982; De Vries et al., 1993). It has been documented that decreased metabolic heat production in summer is due to decreased caloric intake and lowered thyroid hormone production (Baccari et al., 1983). Spiers et al. (2005) reported a $35 \%$ reduction in daily gain from thermoneutrality and suggested that heat stress caused a higher reduction body weight and FI compared eropeptine alkaloids in thermoneutrality. Roberts (2002) also observed a similar phenomenon. Intake of ergopeptine alkaloids in the heat produced the highest decrease in body weight gain compared to either acting alone. The rats that received both stressors lost 20.80 grams each day. Such huge decreases in body weight have been reported previously using the rat model (Spiers et al., 2005; Settivari et al., 2006).

Recent genomic analysis has shown that genes related to protein catabolism and gluconeogenesis were upregulated at thermoneutrality for rats on an endophyteinfected diet (Settivari et al., 2006). Settivari et al. (2006) reported upregulation which include phosphoenolpyruvate carboxykinase and fructose-1-6-biphosphatase. This might help rats consume more feed and adapt better to the toxin. These effects were not seen in the rats exposed to heat stress or combination of heat stress and E+ diet, this might partially explain why these rats did not show any adaptation to the 
toxin. Likewise, FI was also lower in the rats that received ergopeptine alkaloids in the heat and heat stress only, this could have contributed directly to the decreased average daily gain. Once rats start recovering from the reduced FI, the effect of ergopeptine alkaloids on the mitochondrial genes corresponding to ATP production may prevent them from gaining weight (Settivari et al., 2006). This could be the reason, why the average daily gain of $\mathrm{E}+$ rats never reached that of $\mathrm{E}-$ rats in thermoneutral and heat stress conditions.

\section{Average core body temperature}

Average daily core temperature of rats consuming the endophyte-infected fescue diet was lowered at thermoneutrality. Thermoneutral hypothermia was maximal on days six and seven. During this period, the pair-fed rats also had a lower core body temperature compared to rats consuming the uninfected fescue diet. These findings are consistent with another previous report (Spiers et al., 2005). Similarly, hypothermia in response to ergovaline at thermoneutrality has been documented previously (Zhang et al., 1994; Spiers et al., 1995). Zhang et al. (1994) suggested that hypothermia was due to increased peripheral heat loss and decreased metabolic heat production. However, Spiers et al. (2005) showed that ergovaline has direct effects of thermoregulatory system, by demonstrating an immediate decrease in core body temperature at thermoneutral conditions. Pair-feeding causes caloric restriction in rats which can lead to lower body temperature due to decreased metabolic rate and metabolic heat production. Duffy et al. (1989) showed that caloric restriction can reduce core body temperature and related its effects to decreased metabolic rate. $\mathrm{He}$ demonstrated that resting metabolic rate was lower in caloric restricted and starved rats compared to rats that that received ad libitum feed. Similarly, Severinsen and 
Munch, (2001) found that restriction of $25 \%$ the caloric value of the control diet decreased core body temperature by $0.04^{\circ} \mathrm{C}$ for every 24 hours, compared to starved rats that decreased their core body temperature by $0.1^{\circ} \mathrm{C}$ every 24 hours. This shows that magnitude of caloric restriction also impacts core body temperature.

Heat stress significantly increased core body temperatures of all groups in the present study compared to the same treatment groups at thermoneutrality. Likewise, the core body temperature on rats fed the ergot alkaloid diet in the heat was significantly higher than for the other groups. Again, these findings are in agreement with past studies (Spiers et al., 1995; 2005). The increase in core body temperature is due to vasoconstriction of of peripheral blood vessels caused by $\alpha-2$ adrenergic and serotonergic binding (Oliver et al., 1998; Larson et al., 1999). The present short-term heat stress study did not show any adaptation of the elevated core body temperature. This is the first time rats are tested at $33^{\circ} \mathrm{C}$ combined with fescue toxicosis and the increased temperature may thwart adaptive mechanisms. Previous experiments have also shown a partial adaptive response to core body temperature at $32^{\circ} \mathrm{C}$ to $33^{\circ} \mathrm{C}$ when rats were tested with heat stress individually (Gordon, 1993, Horowitz and Meiri, 1993; Matthew, 1997,). Splanchnic and peripheral vasodilation (Horowitz, 1998) helps in heat loss. Likewise, evaporative cooling, increasing surface area by extending limbs and spreading saliva are other ways to reduce skin and ultimately core.body temperature (Schmidek et al., 1983, Hubbard et al., 1982; Gordon., 1993) in rats. Nitric oxide also plays an important role in vasodilation in rats, and the effects may be negated by the toxin. Al-Tamimi et al (2007) showed supplementation of nitric oxide reduces core body temperature. Others hypothesize that interaction with adrenergic and serotonergic receptors causes vasoconstriction and hampers thermoregulatory mechanisms in rats (Oliver, 2005). 


\section{General activity}

General activity of rats in the present study was highly variable among treatment groups. However, no significant difference was observed across treatment groups during pretreatment and diet treatments. These findings are in agreement with previous reports (Spiers et al., 2005). In this study, heat stress generally reduced activity in rats. Spiers et al. (2005) stated that a reduced decreased activity is an attempt to lower core body temperature. Others have shown that rats in the heat have reduced activity and decreased metabolic rate (Shido et al., 1991; Gordon, 1993) The rats consuming the control diet had a higher activity in the heat compared to the animals on the ergopeptine alkaloid infected diet in the heat. It is possible that this reduced activity represents a form of sickness behaviour. Previous research has also that sick rats have lowered activity compared to healthy rats, due to both the infections and psychological disorders associated with illness (Meerlo et al., 1996; Luker et al., 2000). Therefore, we suggest that lower activity in E+ HS groups is an indication of sickness behaviour. Increase in activity observed in E-HS is a new observation. This could be due to high levels of heat stress, and rats might seek to burrow or may try to find a place away from the heat.

\section{Circadian changes in core body temperature and activity}

Higher core body temperatures were observed in rats during the night. Rats are nocturnal animals, and the increase in core body temperature is attributed to their natural behaviour in the wild. Scales and Kluger, (1987) reported similar changes in the circadian rhythm of rats. Likewise, daily feeding time resulted in a spike in core body temperature. It is suggested that feed consumption results in increased heat 
production as a consequence of metabolic heat production in the body. Similarly, activity of rats can be connected to core body temperature. Both followed the same increases and decreases during a 24 hour period. In this experiment, activity was highest at night when lights were turned off, and these findings are consistent with previous reports (Sevrinsen and Munch, 1999; Spiers et al., 2005). Likewise, a significant decline in activity was recorded before lights were turned off. Similar reductions have been reported (Spiers et al., 2005).

\section{Lymphocytes}

\section{Natural killer cells}

Flow cytometric analysis showed an increase in the natural killer activity of all groups used in the present study, except the control group at thermoneutrality. These findings are consistent with the previous findings which show that stress and caloric restriction increase natural killer cell percentages and activity (Biron, 1997; Shibolet et al., 2000; Shen et al., 2009). The present findings suggest that rats may activate their immune system, in anticipation of an infection (Biron et al., 1987; Biron and

Orange, 1996). Rats exposed to duel stressors (i.e., heat stress and fescue toxicosis) had the greatest increase in NK percentages. We hypothesize that intensity of stress may also influence the magnitude of NK activation and response. This is evident from the increased, additive activation found in rats consuming the endophyteinfected diet in the heat, which received the highest level of stress. This increase might be a host defense mechanism to counter invading pathogens in times of extreme stress (Biron, 1997). 


\section{B-lymphocytes}

Lymphocyte percentage across treatment groups was fairly constant except for a significant reduction in these levels for the rats consuming the endophyte-infected diet in the heat. This is in agreement with an earlier study that showed a decrease in Bcell percentages in response to extreme stress (Dracott and Smith, 1979). Likewise, previous studies have shown that both short and long-term treatment with fescue toxin did not affect the animals ability to mount an inflammatory or antibody response to these stressors (Rice et al.,1997; Filipov et al., 1999a). Since B cells are involved in antibody production, we suggest that major alterations may not be found in antibody production for rats fed the endophyte-infected diet at thermomeutrality. Interestingly, rats fed the infected diet in the heat had the lowest B-lymphocyte percentages. Therefore, we propose that rats ability to produce antibodies may be compromised in this treatment group..

\section{T-lymphocytes}

The T-lymphocyte percentage for animals in the present study varied across treatment groups. There was a marked increase in rats receiving the endophyteinfected diet in the heat, at the same time that they experienced a suppression in the B cell percentage. Conversely, animals fed the endophyte-infected diet at thermoneutrality had a significantly lower percentage of T-lymphocytes than the heat stressed animals on the same treatment diet. Settivari et al. (2006) reported down regulation of TCR beta genes in response to endophyte infected fescue. Earlier reports have stated that feeding mice an endophyte-infected diet at thermoneutrality increased T-suppressor cell percentage at the end of fourty three days (Dew et al., 1990). Dew et al. (1990) suggested that this activation may decrease T-cell 
percentage and activity, however, the total T-lymphocyte percentage was not measured in that study. The increased percentage of $\mathrm{T}$ cells in the heat stressed rats consuming the endophyte-infected diet is a new finding, and it could be an initial response to the extreme levels of stress. However, it is important to determine if $\mathrm{T}$ cell percentages change over a longer period of exposure.

\section{CD4+ and CD8+ cells}

The CD4+ CD8+ percentages have been used by others in the diagnosis of immunosuppressive conditions like HIV (Taylor et al., 1989). Many studies have reported that these percentages can change in chronic immunosuppressive diseases and percentages are a better prognostic indicator compared to total count (Taylor et al., 1989; Burcham et al., 1991, Ander Ryst et al., 1998). In the present study, CD4+ and CD8+ cells did not differ significantly across treatment, because of the short period of stress.

\section{Core body temperature and activity in response to LPS}

The febrile response to LPS injection in rats is controversial and it has been debated for years. In our studies, rats did not increase their core body temperature significantly following injection and actually showed a trend towards hypothermia at thermoneutrality, which is a popular and widely accepted phenomenon (Kasting et al., 1973; Kasting and Martin, 1983; Derijk and Berkenbosch, 1994; Romanovsky et al., 1996; Chu et al., 1997; Bison et al., 2009). A few studies have noted that rats increase their core body temperature in response to heat stress and LPS (Kluger et al., 1997). In these studies rats were heat stressed prior to LPS and the administered LPS and reported an increase in core body temperature in response to LPS. In our study rats 
were continuously exposed to heat and we observed at most only a trend towards hyperthermia. Similar findings have been reported in the past, were prior heat stress increased core body temperature of rats (Kluger et al 1997; Chu et al 1997). Similar to our studies, Chu et al. (1997) observed a decrease in core body temperature of rats at thermoneutrality and an increase during heat stress immediately after an LPS challenge. Some of their findings are significant, and this could be due to high LPS dose $(15 \mathrm{~g} / \mathrm{kg} \mathrm{BW})$ compared to the $100 \mu \mathrm{g} / \mathrm{kg}$ BW dose used in our study. This study clearly shows that fever in rats may differ in relation to environmental temperature. Likewise, the dose and route of administration of LPS may plays a major role in determining the nature of response. In a recent rat study, LPS doses (IP) from 1 to $250 \mu \mathrm{g} / \mathrm{kg} \mathrm{BW}$ were used for a challenge, and animals were monitored over a period of 24 hours to measure core body temperature, activity, and cytokine response (Bison et al., 2009). No significant change in core body temperature was noted in the present study for the first two hours, until a dose of $250 \mu \mathrm{g} / \mathrm{kg} \mathrm{BW}$ was used. The same study reported fever after 24 hours in rats that received $125 \mu \mathrm{g} / \mathrm{kg} \mathrm{BW}$. In our study, there was a trend towards decreased activity after an hour of LPS challenge and Bison et al. (2009) reported significant decrease in the activity of rats 24 hours after LPS challenge. There is no information available on the effects of LPS on rats fed an endophyte- infected diet in the heat on core body temperature and activity. From our results, it is suggested that intake of an endophyte-infected diet or caloric restriction may have limited impact on core body temperature and activity in the rat model.

\section{Cytokine response to LPS}

Although core body temperatures did not differ significantly with an LPS challenge in the present study, there was a significant cytokine response. All 
treatment groups retained the ability to produce pro-inflammatory cytokines following the challenge, and this is in agreement with other studies (Rice at el., 1997; Filipov et al., 1999b). The rats fed the endophyte-infected diet in the heat showed the greatest increase in IL1 concentration, to suggest that the increased stress experienced by these animals had a significant effect. Increased pro-inflammatory response to endotoxin at thermoneutrality has been reported for mice and cattle on an endophyte-infected diet (Rice at el., 1997; Filipov et al., 1999a, Filipov et al., 1999b). Other studies have shown that caloric restriction of $40 \%$ for four to seven months can significantly reduce the proinflammatory response (Muthukumar et al., 2000). The results from the present study only showed a diminished response for rats that were pair-fed in heat stress chambers. We attribute this to the lower FI of heat-stressed rats fed the endophyte-infected fescue compared to rats at thermoneutrality. The animals that were pair-fed to this group would likely be in a starvation mode with significant caloric restriction. Rats fed an endophyte-infected fescue at thermoneutrality showed partial recovery of FI, so the beneficial effects associated with caloric restriction may have been counteracted. Both groups fed the uninfected diet and those pair-fed to those fed the infected diet under heat stress conditions had a lower IL1 response compared to those on the same diet treatment at thermoneutrality. This same trend has been reported previously (Chu et al., 1997). This is considered to be a protective and adaptive mechanism to survive heat stress. Chu et al. (1997) reported increased survival of heat stressed rats compared to thermoneutral groups, when challenged with LPS. The rats exposed to heat stress on the uninfected diet had a higher level of TNF $\alpha$, as reported by others (Bouchama et al., 1991; Lin et al., 1997). Interestingly, $\mathrm{TNF} \alpha$ levels were lower in rats fed endophyte-infected diet in the heat and highest in rats that were subject to heat stress only. The TNF $\alpha$ levels may have significantly 
risen immediately after LPS challenge, and this triggered an increase in IL1 (Bison et al., 2009). Our samples were collected s at two hours, when TNF $\alpha$ levels may have dropped. Zuckerman and Butter, (1989) reported that TNF $\alpha$ levels peak approximately at one hour after LPS challenge and then decreases to baseline level at three to four hours after LPS challenge. In contrast, IL1 levels remained higher for 24 hours after LPS challenge. He also reported that cortisol or dexamethasone, associated with the stress response, can rapidly decrease TNF $\alpha$ levels. In our shortterm study, extreme stress caused by intake of the endophyte-infected diet, heat stress, and then the LPS injection could have triggered a massive release of cortisol, blunting TNF $\alpha$ levels rapidly. The very high level of IL1 in E+HS was another indication that the rats experienced a massive stress load.

\section{Summary}

The current study has provided a broad overview of the immune system shortterm response to intake of an endophyte-infected diet, heat stress, and caloric restriction. The results have helped to differentiate the effects of individual stressors and interactions of stressors on different physiologic parameters that are associated with various components of the immune system. Short-term studies revealed that the immune system of rats fed an endophyte infected diet during heat stress was affected the most. These animals responded with a greater immune response during short-term stress. This study reveals that either heat stress or endophyte alone may not significantly impact the immune system. Long-term studies are now needed to understand the complex pattern of adaptation to fescue toxicosis during heat exposure. 


\begin{tabular}{ll|l|l|l|}
\hline \multirow{2}{*}{ PERIODS } & $\begin{array}{l}\text { TN } \\
\text { Baseline }\end{array}$ & Diet Trt & $\begin{array}{l}\text { Diet }+ \\
\text { TaTrt }\end{array}$ & LPS \\
\cline { 2 - 5 } DAYS & -5 to -1 & 1 to 7 & 8 to 10 & 11 \\
Ta & $21^{\circ} \mathrm{C}$ & $21^{\circ} \mathrm{C}$ & $21^{\circ} \mathrm{C} / 33^{\circ} \mathrm{C}$ & \\
\hline
\end{tabular}

Figure 2. Timeline of short term study. Time line of study shows E+, E- and PF TO E+ rats maintained in pretreatment, diet treatment and thermal treatment. The timeline also shows the number of days that rats were placed on each of these stressors and the temperature of chambers during the specific treatment periods. 


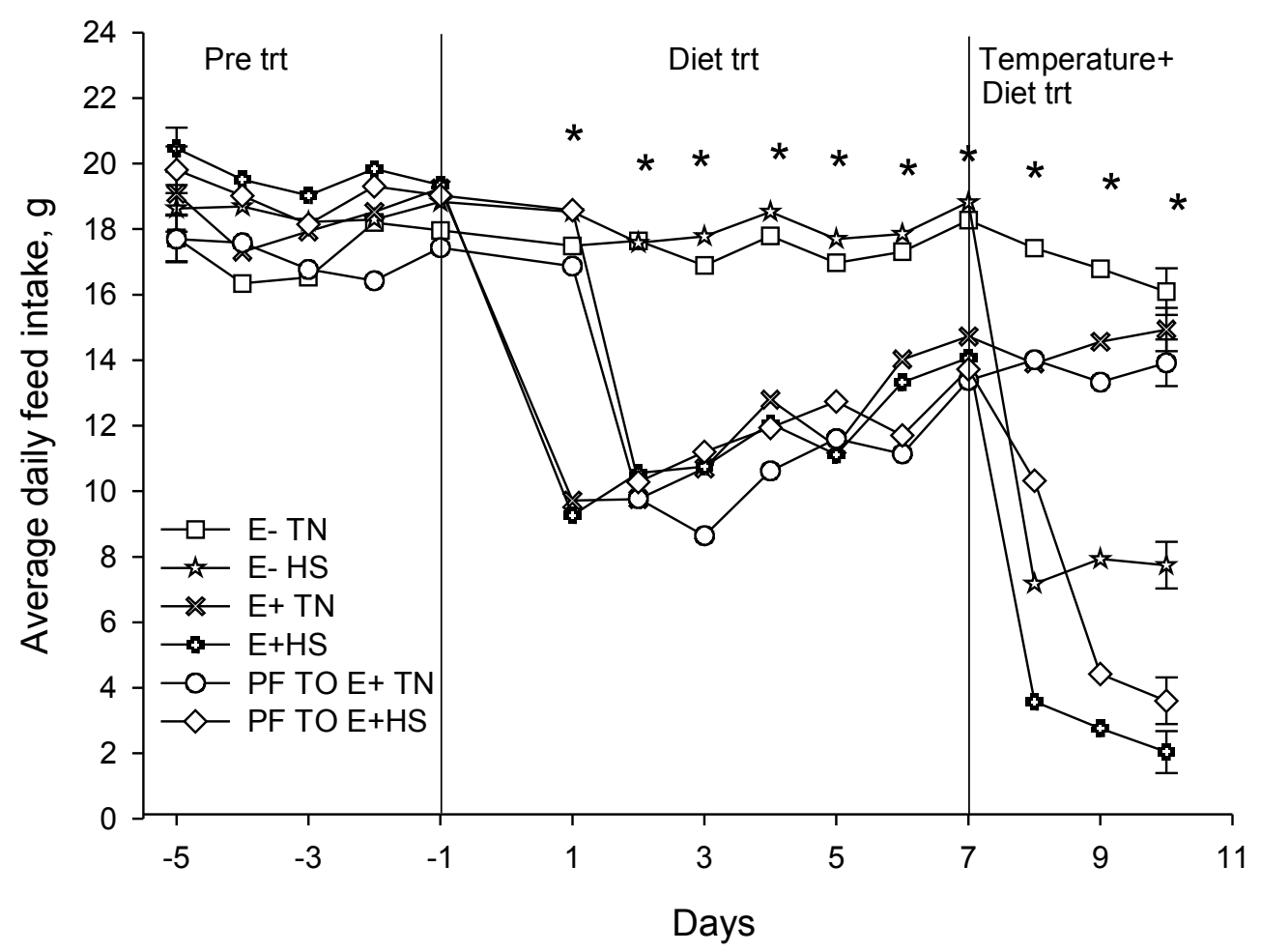

Figure 2.1. Average daily feed intake of E-, E+, and PF TO E+ rats during HS and TN periods. An asterisk $(*)$ shows the statistical significance of $\mathrm{E}+\mathrm{HS}$ and PF TO E+ HS compared to E-TN treatment. The vertical lines above and below the first and last points indicate \pm SE. 


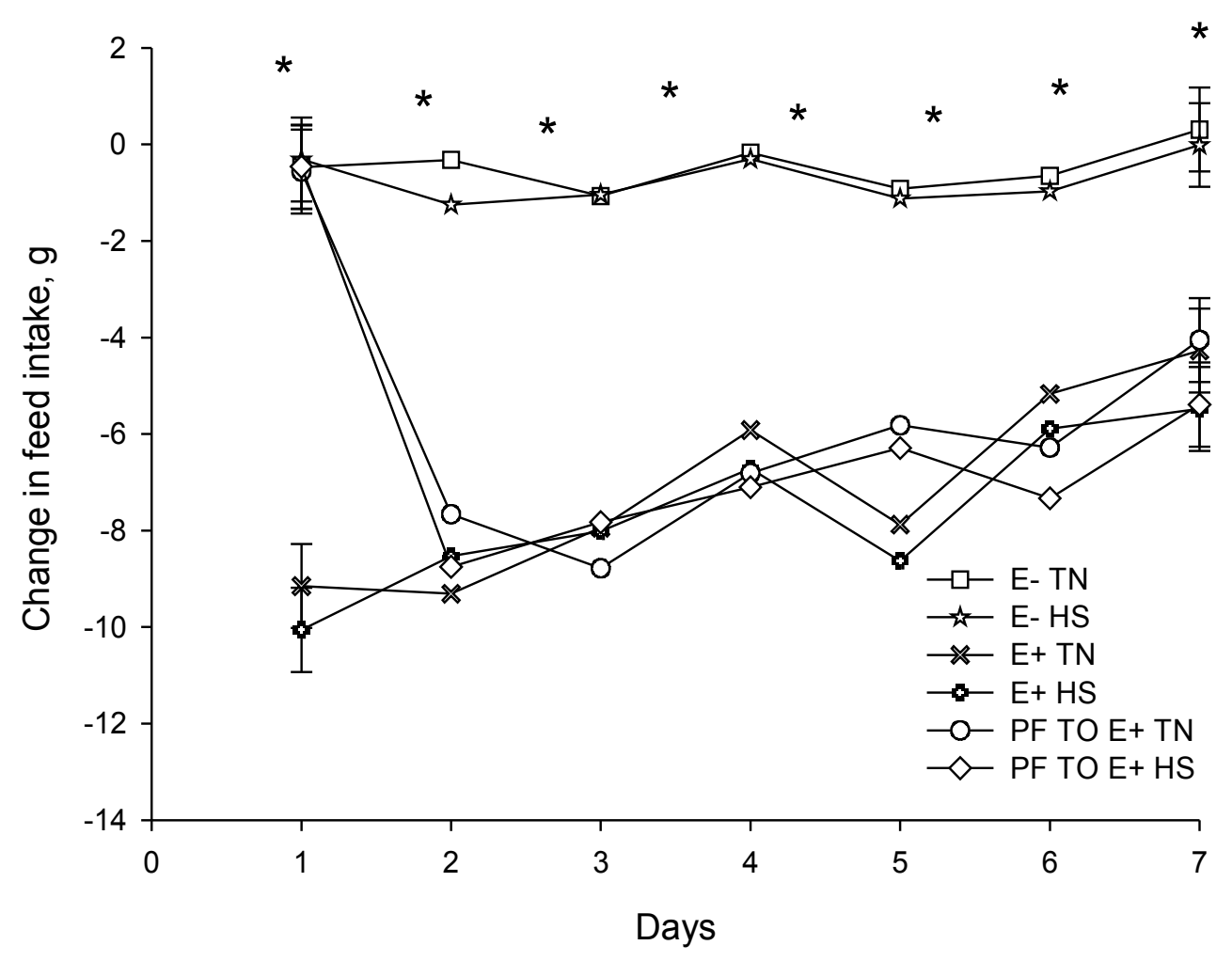

Figure 2.2. Change in average daily feed intake of E-, E+, and PF TO E+ during pretreatment period (Days 1 to 7 ). E- TN rats consumed $18 \mathrm{~g}$ on day -1 . An asterisk (*) shows the statistical significance of $\mathrm{E}+$ and $\mathrm{PF}$ TO E+ compared to E-TN rats. The vertical lines above and below the first and last points indicate + SE. The vertical lines above and below the first and last points indicate \pm SE. 


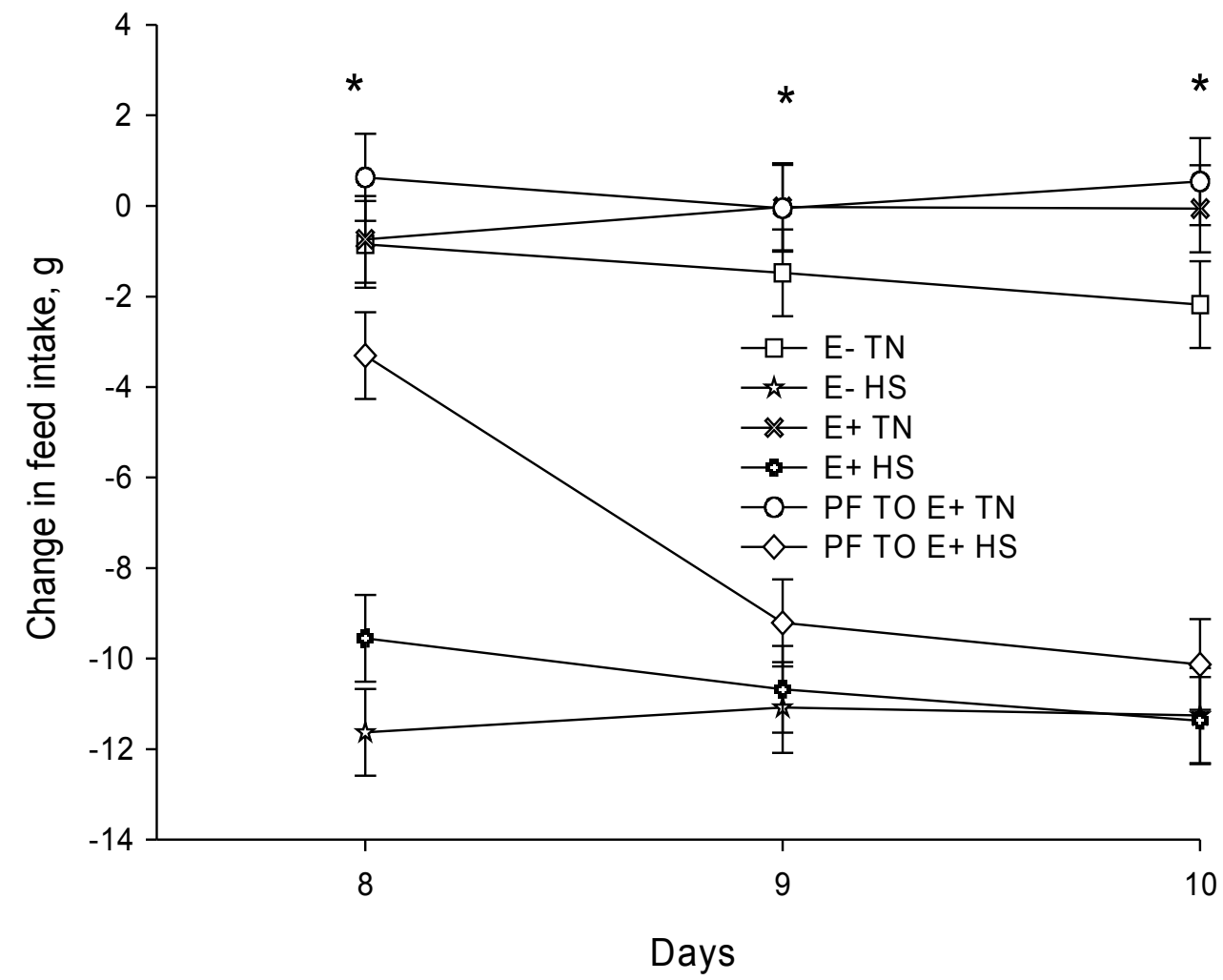

Figure 2.3. Change in average daily feed intake of E-, E+, and PF TO E+ during thermal treatment period (Days 8 to 10 ). E- TN rats consumed $18 \mathrm{~g}$ on day 7 .An asterisk $(*)$ shows the statistical significance of $\mathrm{E}+$ and $\mathrm{PF}$ TO E+ compared to E-TN rats. The vertical lines above and below the first and last points indicate \pm SE. 


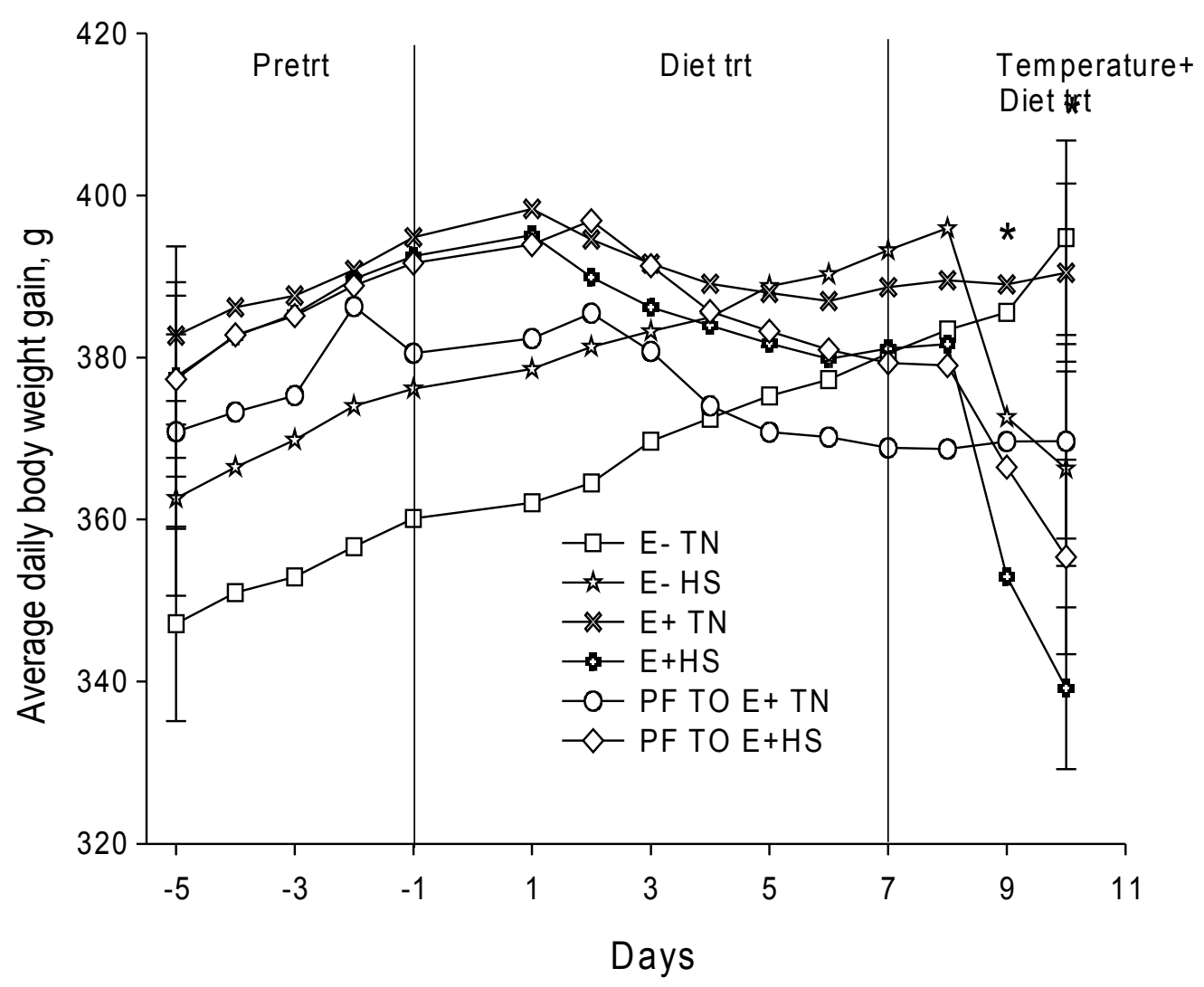

Figure 2.4. Change in average daily body weight gain of E-, E+ and PF TO E+ rats maintained at $\mathrm{HS}$ and TN conditions during the entire study. An asterisk $\left({ }^{*}\right)$ shows the statistical significance between E+ HS and E-TN rats. The vertical lines above and below the first and last points indicate \pm SE. 


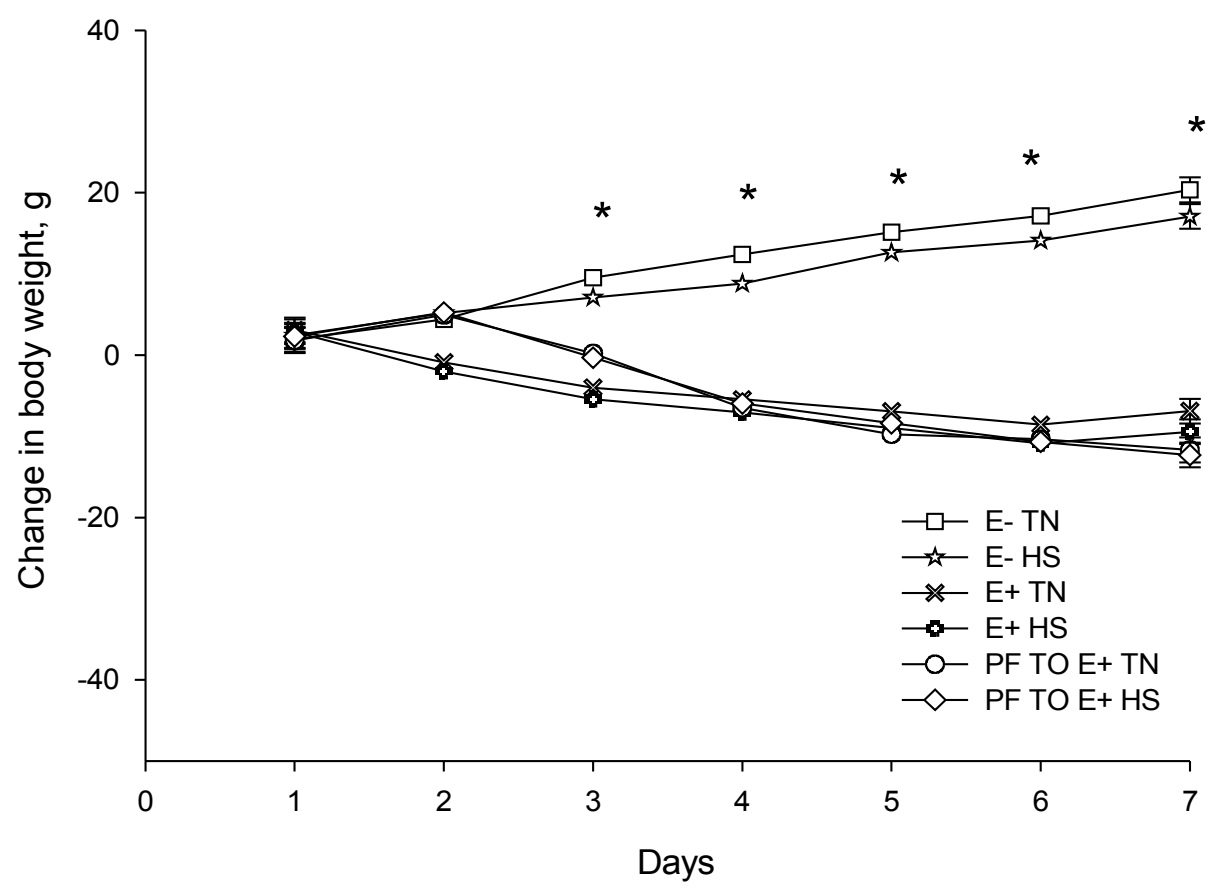

Figure 2.5. Change in average daily body weight of E-, E+, and PF TO E+ groups during diet treatment at TN (Days 1 to 7 ). An asterisk $\left(^{*}\right)$ shows the statistical significance between $\mathrm{E}+\mathrm{TN}$ and E-TN groups. The vertical lines above and below the first and last points indicate \pm SE. 


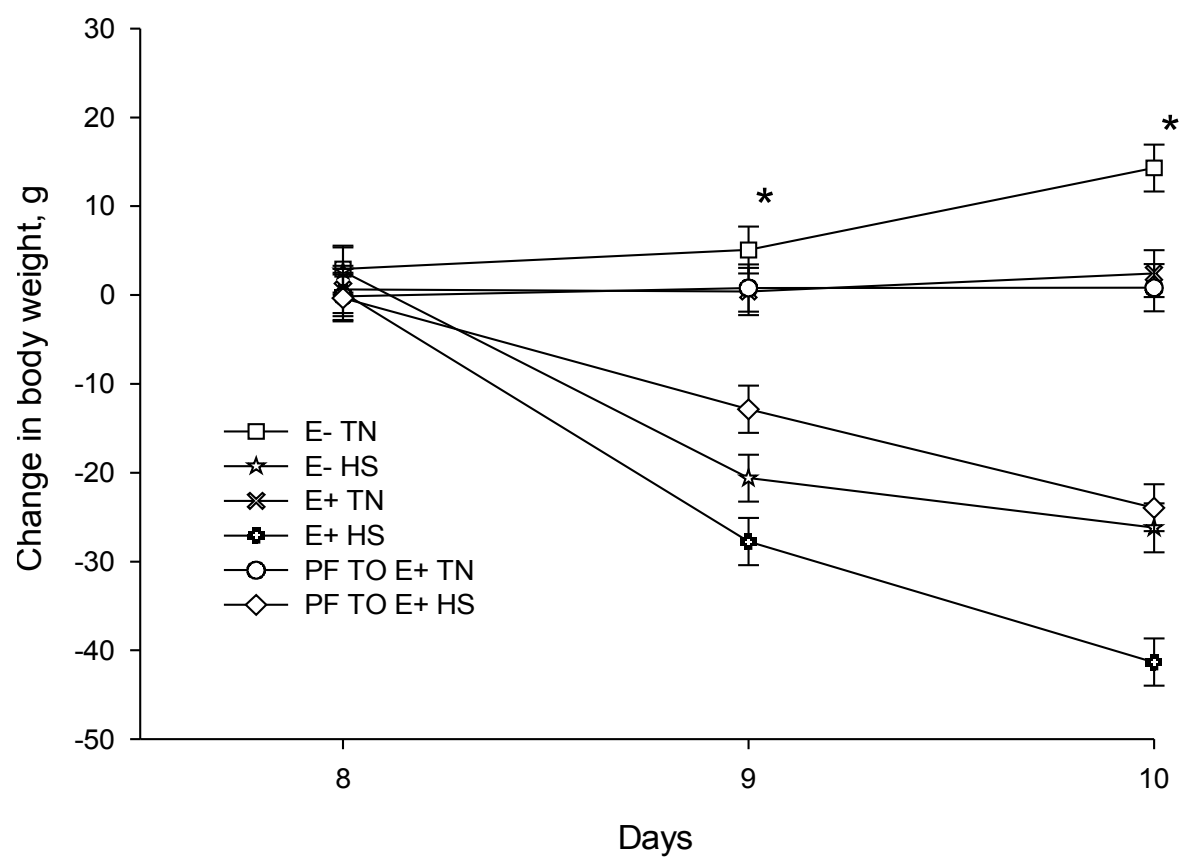

Figure 2.6. Change in average daily body weight of E-, E+, and PF TO E+ during thermal and diet treatment period (Days 8 to 10). An asterisk (*) shows the statistical significance between E+ HS and E-TN rats. The vertical lines above and below the first and last points indicate \pm SE. 


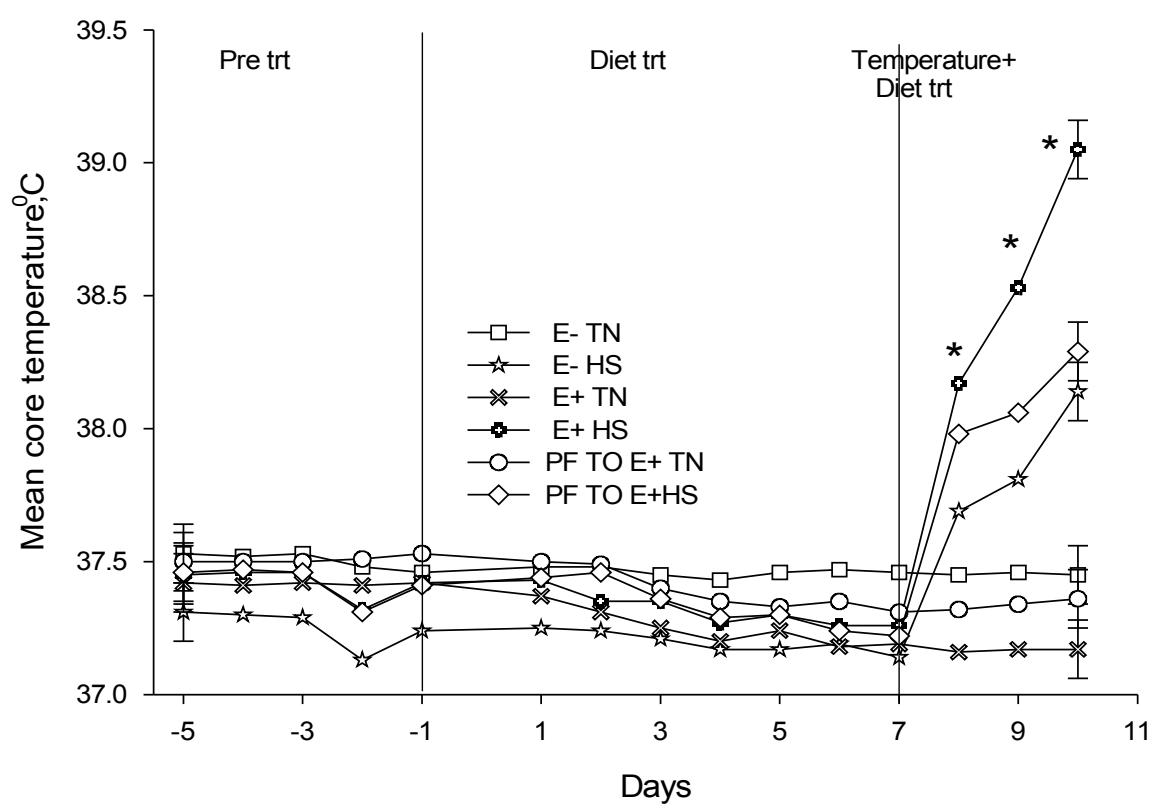

Figure 2.7. Change in average core body temperature of E-, E+, and PF TO E+ rats maintained under HS and TN conditions. An asterisk (*) shows the statistical significance between E+HS and E-TN rats. The vertical lines above and below the first and last points indicate \pm SE. 


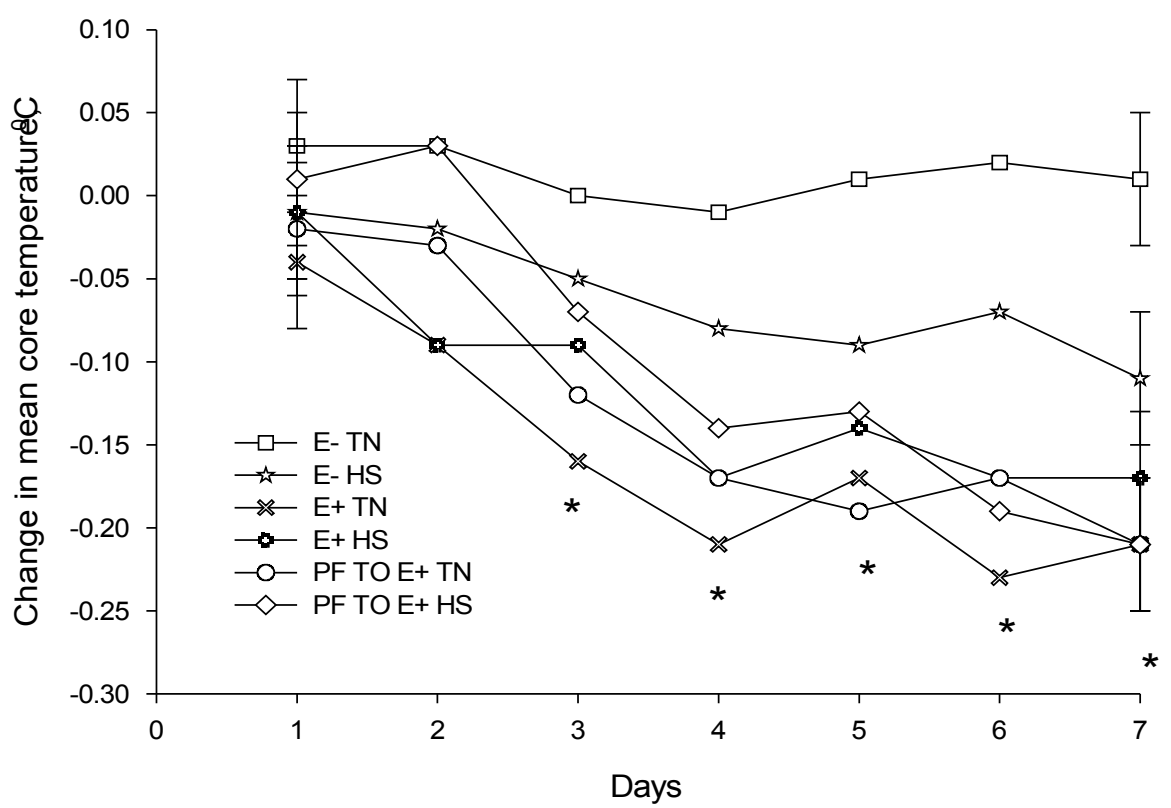

Figure 2.8. Change in average core temperature of E-, E+, and PF TO $\mathrm{E}+$ during the diet treatment period (Days 1 to 7). An asterisk (*) shows the statistical significance of E+ TN compared to E-TN rats. The vertical lines above and below the first and last points indicate \pm SE 


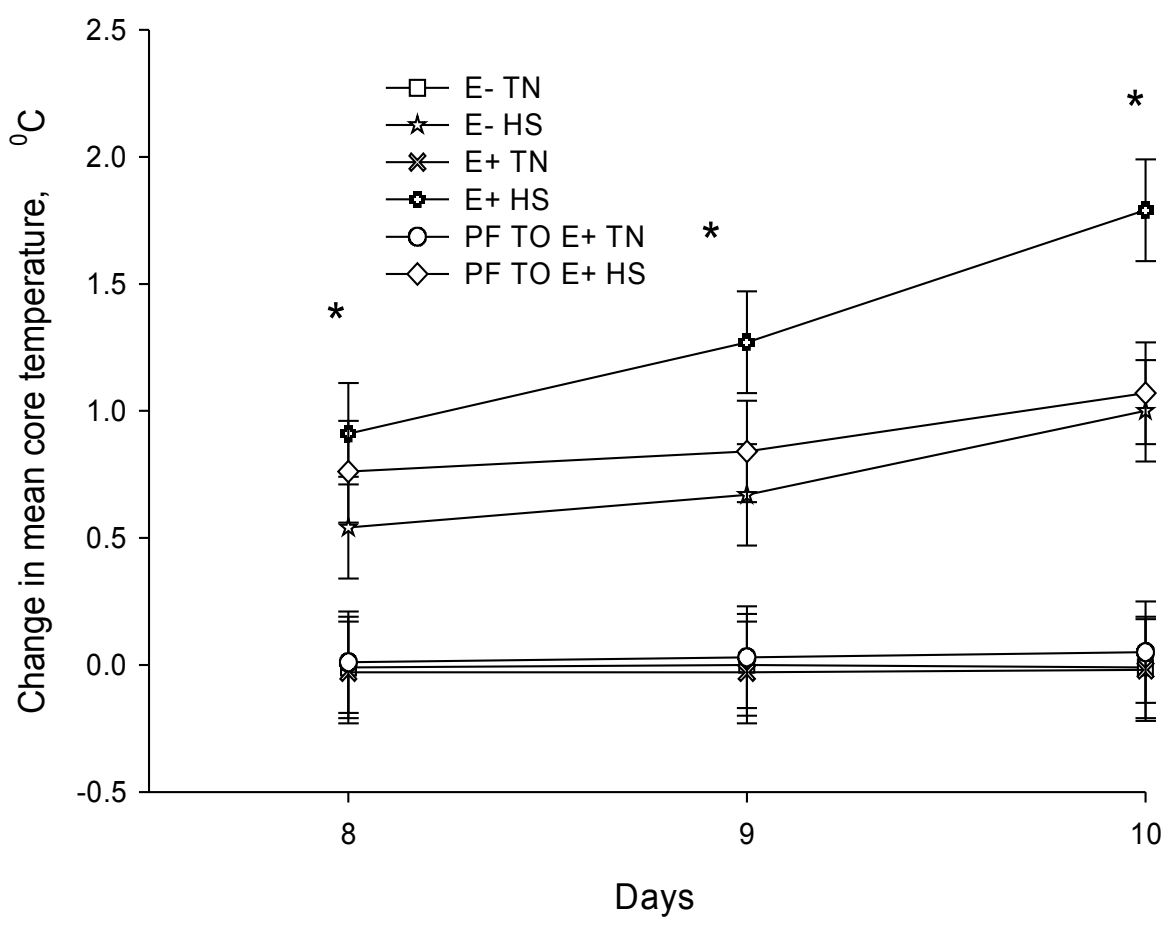

Figure 2.9. Change in average core body temperature of E-, E+ and PF TO E+ during the thermal and diet treatment period (Days 8-10). An asterisk $(*)$ shows the statistical significance between heat stressed and thermoneutral treatment groups. The vertical lines above and below the first and last points indicate $\pm \mathrm{SE}$ 


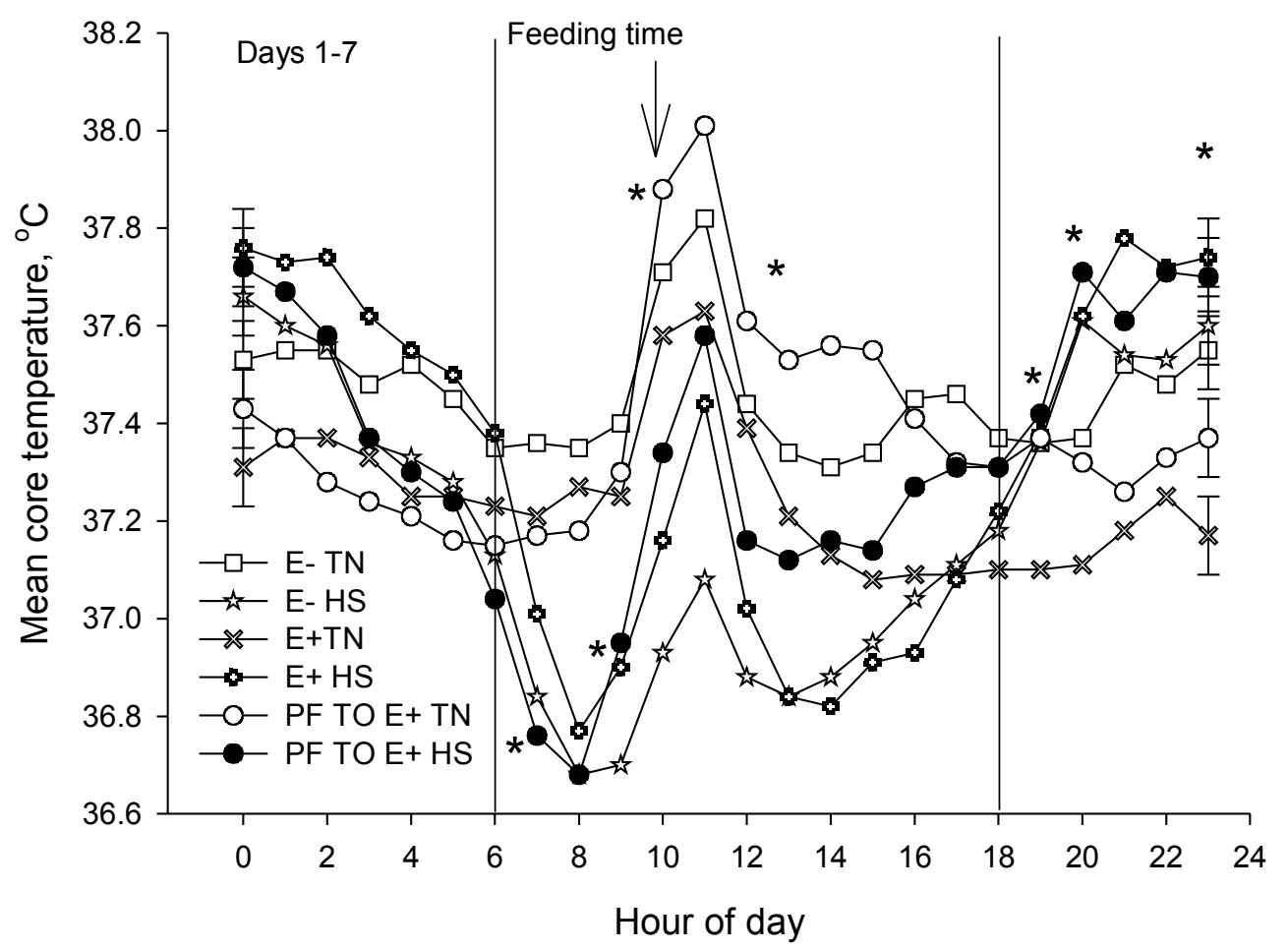

Figure 2.10. Circadian changes in average core body temperature of E-, E+, and PF TO E+ during the diet treatment period (Days 1 to 7). Vertical lines show periods when lights were turned on (0600) and when lights were turned off (1800). An asterisk $(*)$ shows the statistical significance from previous hour of day. The vertical lines above and below the first and last points indicate \pm SE. 


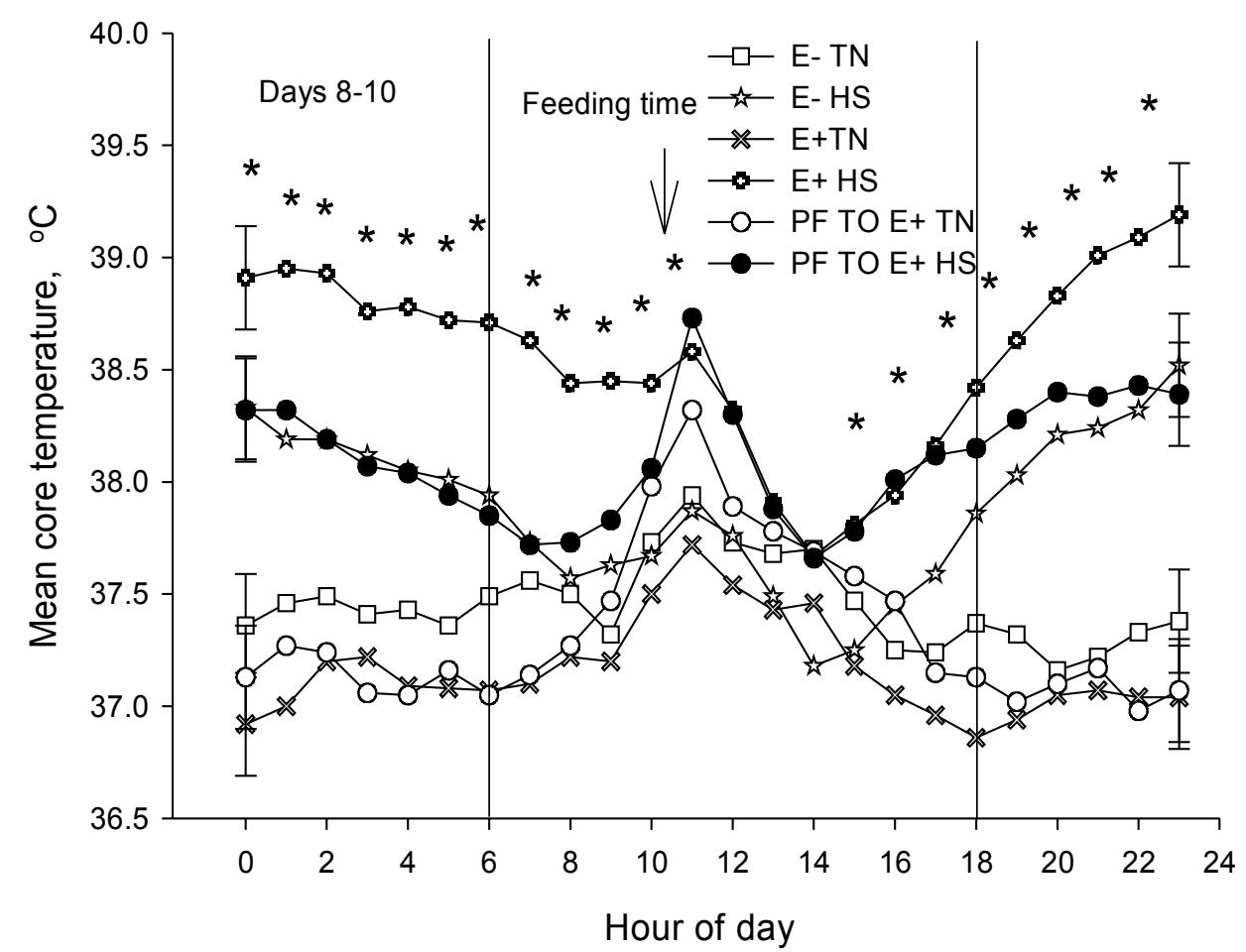

Figure 2.11. Circadian changes in average core body temperature of E-, E+, and PF TO E+ during the thermal and diet treatment period (Days 8 to 10). Vertical lines show periods when lights were turned on (0600) and when lights were turned off (1800). An asterisk (*) shows the statistical significance between E+ HS and E-TN rats. The vertical lines above and below the first and last points indicate $\pm \mathrm{SE}$. 


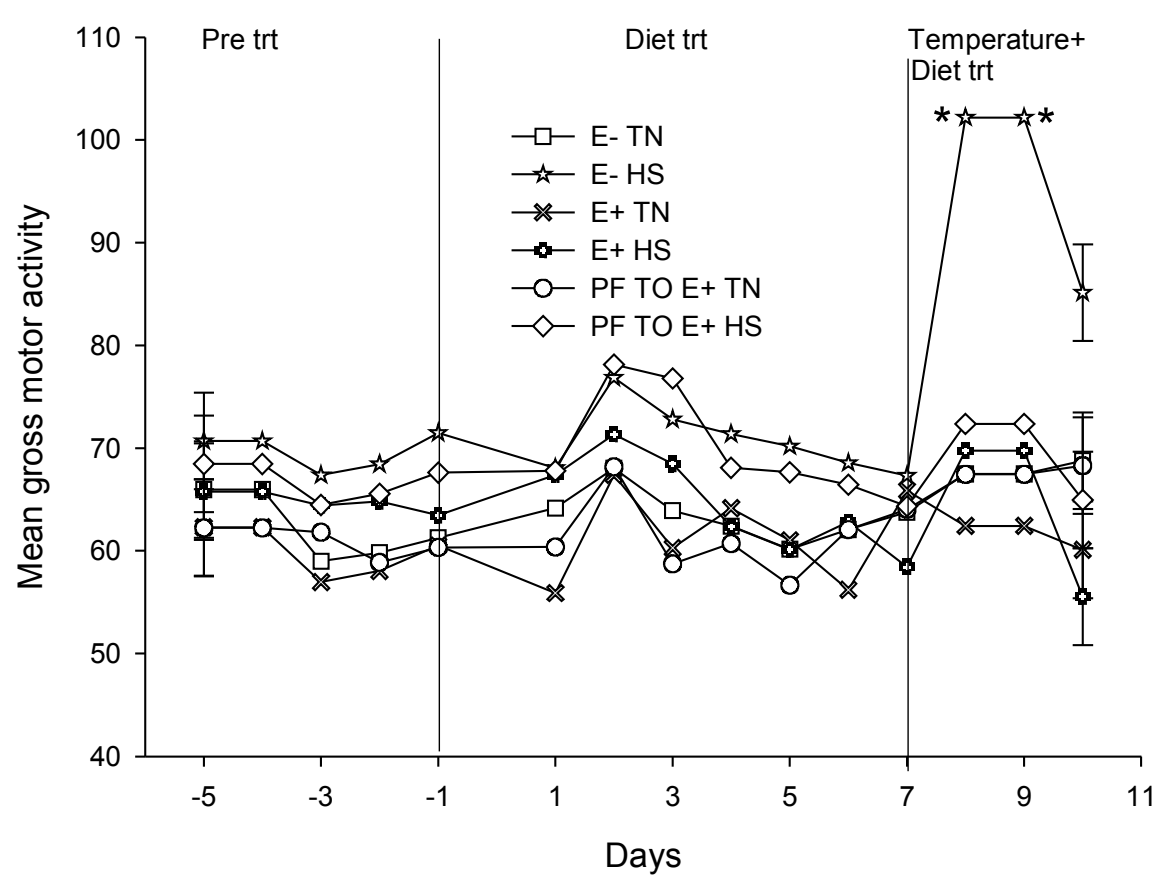

Figure 2.12. Mean gross motor activity of E-, E+, and PF TO E+ groups during the thermal and diet treatment period. An asterisk (*) shows the statistical significance between E- HS and E-TN rats. The vertical lines above and below the first and last points indicate \pm SE. 


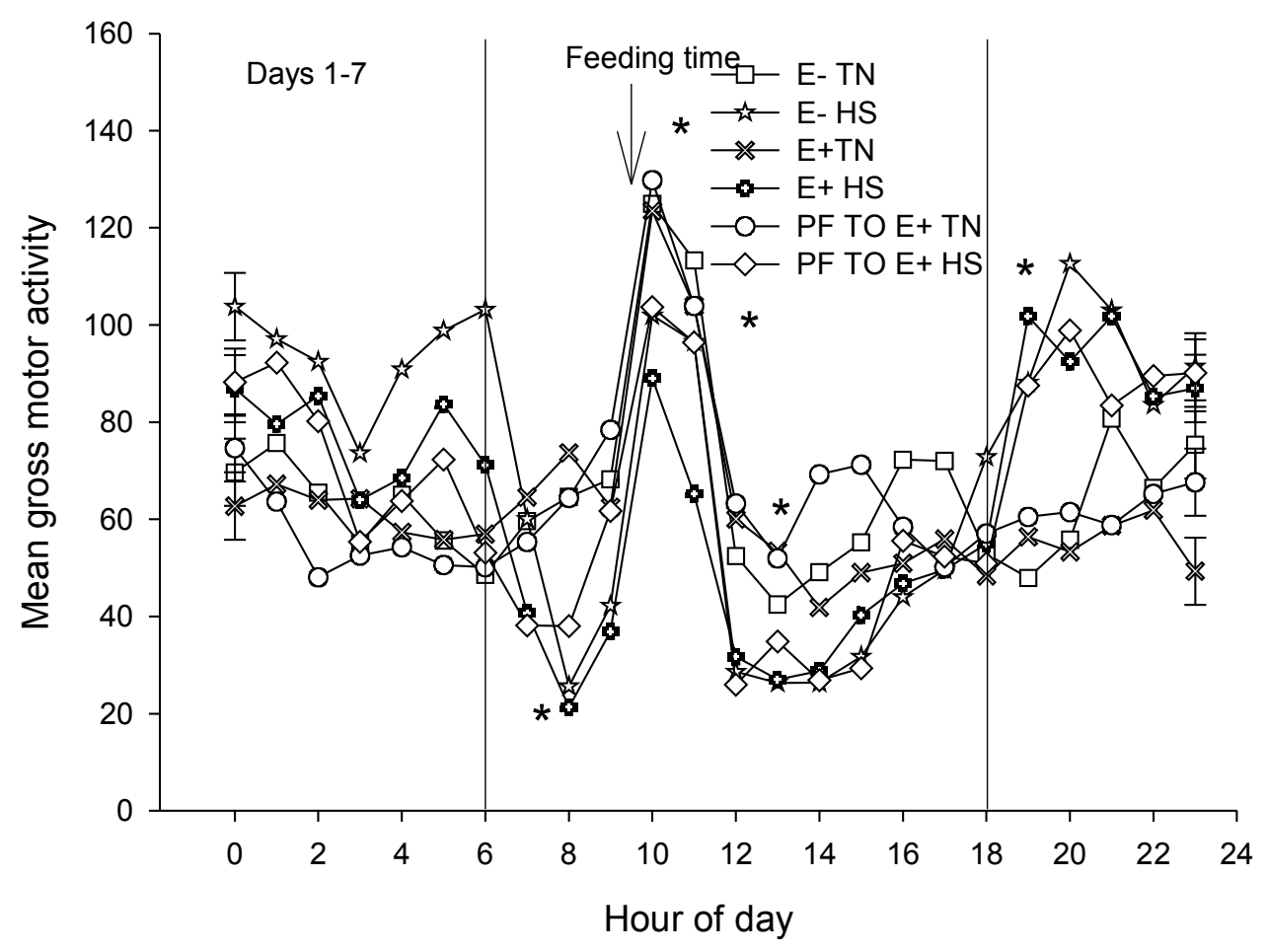

Figure 2.13. Circadian changes in gross motor activity of E-, E+, and PF TO $\mathrm{E}+$ groups during the diet treatment period (Days 1 to 7 ). Vertical lines show periods when lights were turned on (0600) and when lights were turned off (1800). An asterisk $(*)$ shows the significant change from previous hour of day. The vertical lines above and below the first and last points indicate \pm SE. 


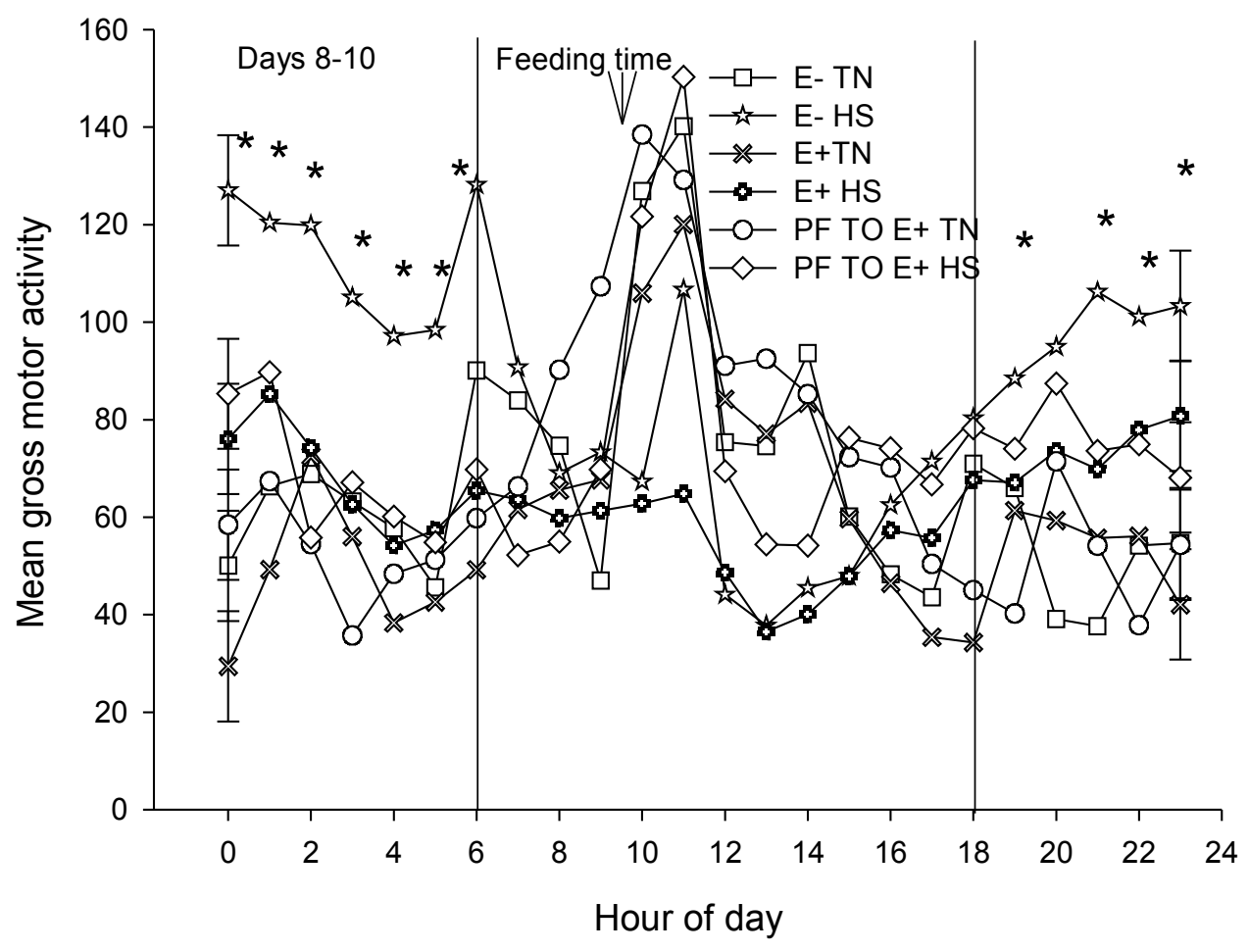

Figure 2.14. Circadian changes in gross motor activity of E-, E+, and PF TO $\mathrm{E}+$ groups during the thermal treatment period (Days 8 to 10 ). Vertical lines show periods when lights were turned on $(0600)$ and when lights were turned off (1800). An asterisk $(*)$ shows the significant difference between E+ HS and E- TN rats. The vertical lines above and below the first and last points indicate $\pm \mathrm{SE}$ 


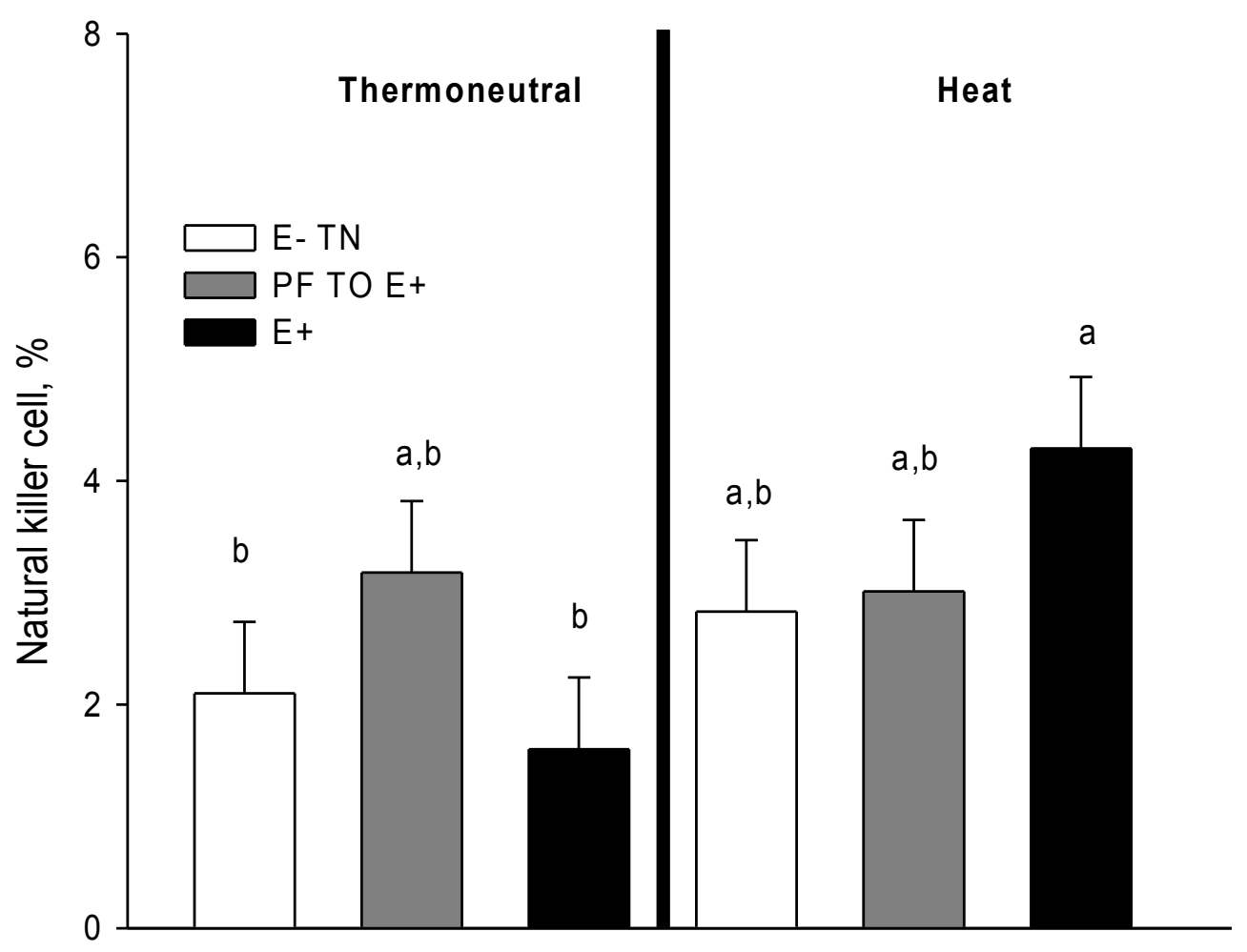

Figure 2.15. Horizontal bars represent E-, E+, and PF TO E+ percentages of natural killer cells under HS and TN conditions. Different letters indicate significant differences across treatment groups. Heat $(\mathrm{P}>0.06)$ and endophyte $(P>0.58)$ individually did not have any significant effects on NK cells 


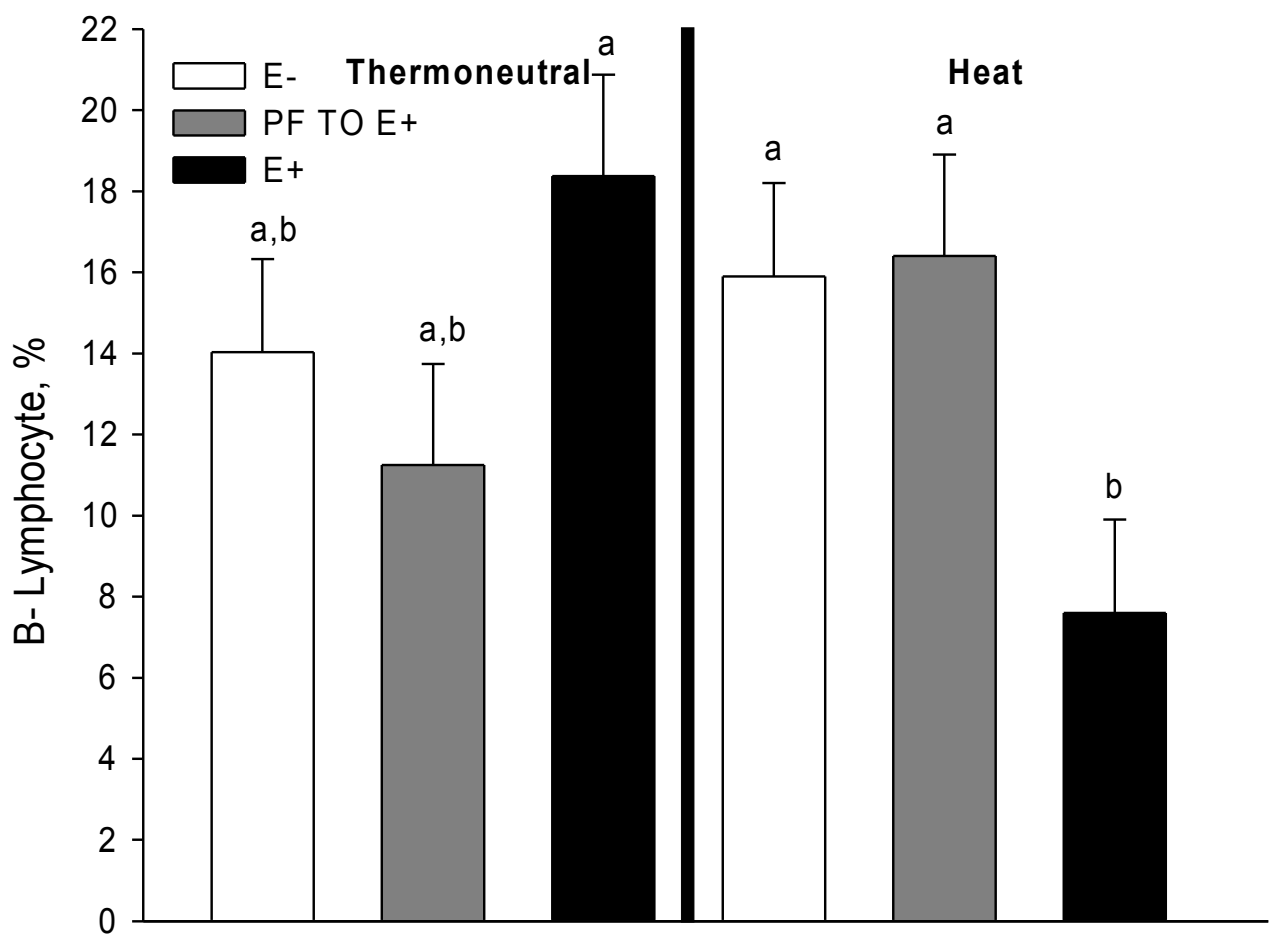

Figure 2.16. Horizontal bars represent E-, E+, and PF TO E+ percentages of B-lymphocytes cells under HS and TN conditions. Different letters indicate significant differences across treatment groups. Heat $(\mathrm{P}>0.67)$ and endophyte $(\mathrm{P}>0.66)$ individually did not have any significant effects on B-lymphocytes. 


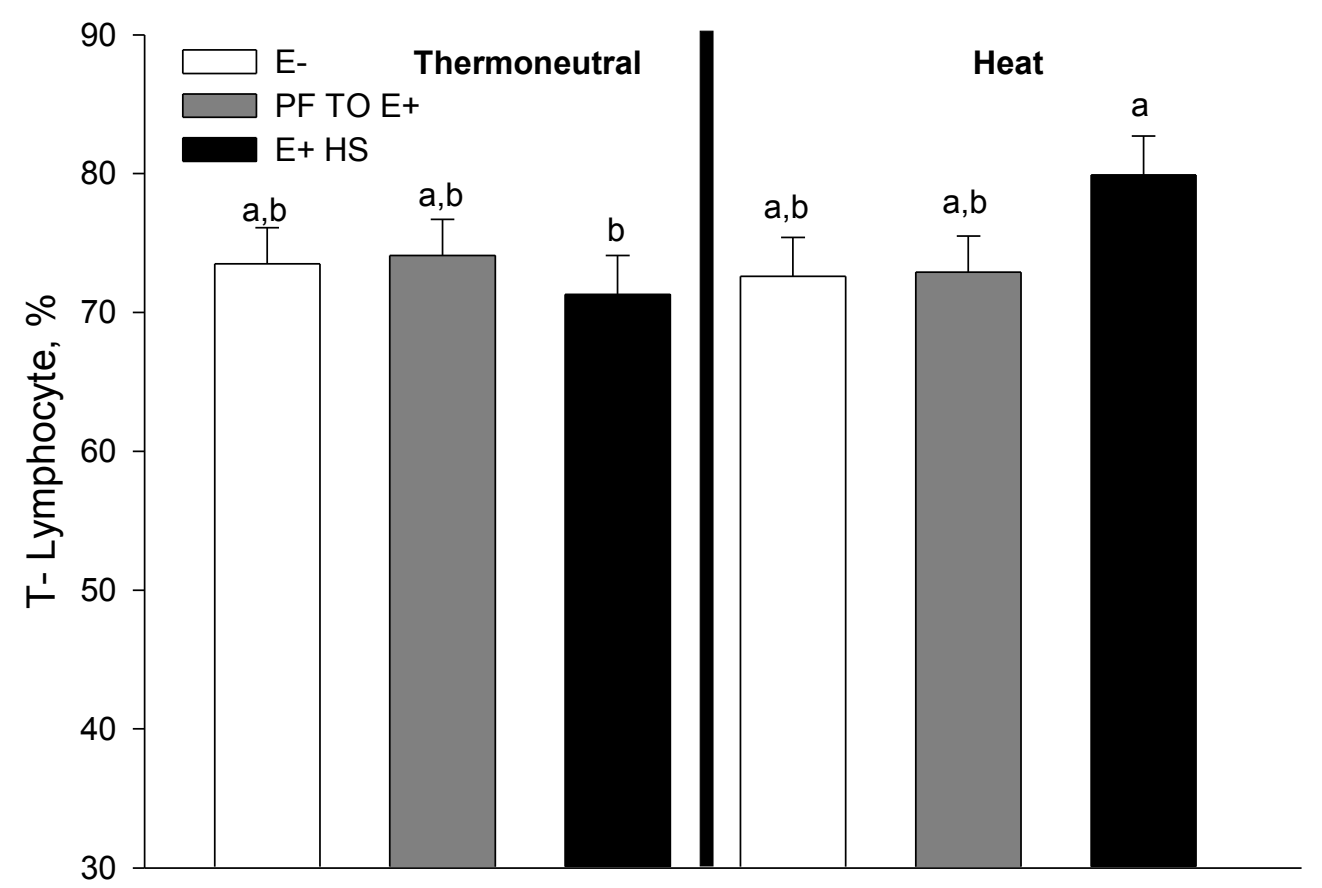

Figure 2.17. Horizontal bars represent E-, E+, and PF TO E+ percentages of Tlymphocytes for rats under HS and TN conditions. Different letters indicate significant differences across treatment groups. Heat $(\mathrm{P}>0.34)$ and endophyte $(\mathrm{P}>0.64)$ individually did not have any significant effects . 


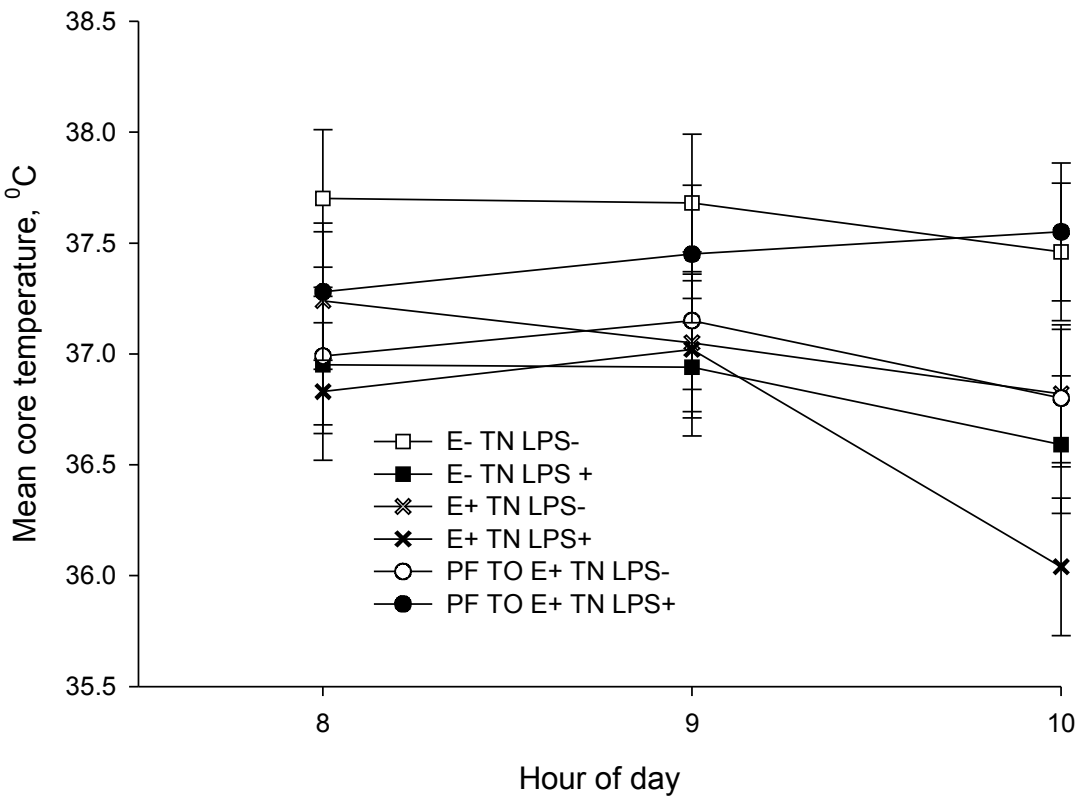

Figure 2.18. Change in average core body temperature of E-, E+ and PF TO E+ in response to LPS challenge at thermoneutrality. The vertical lines above and below the first and last points indicate \pm SE. 


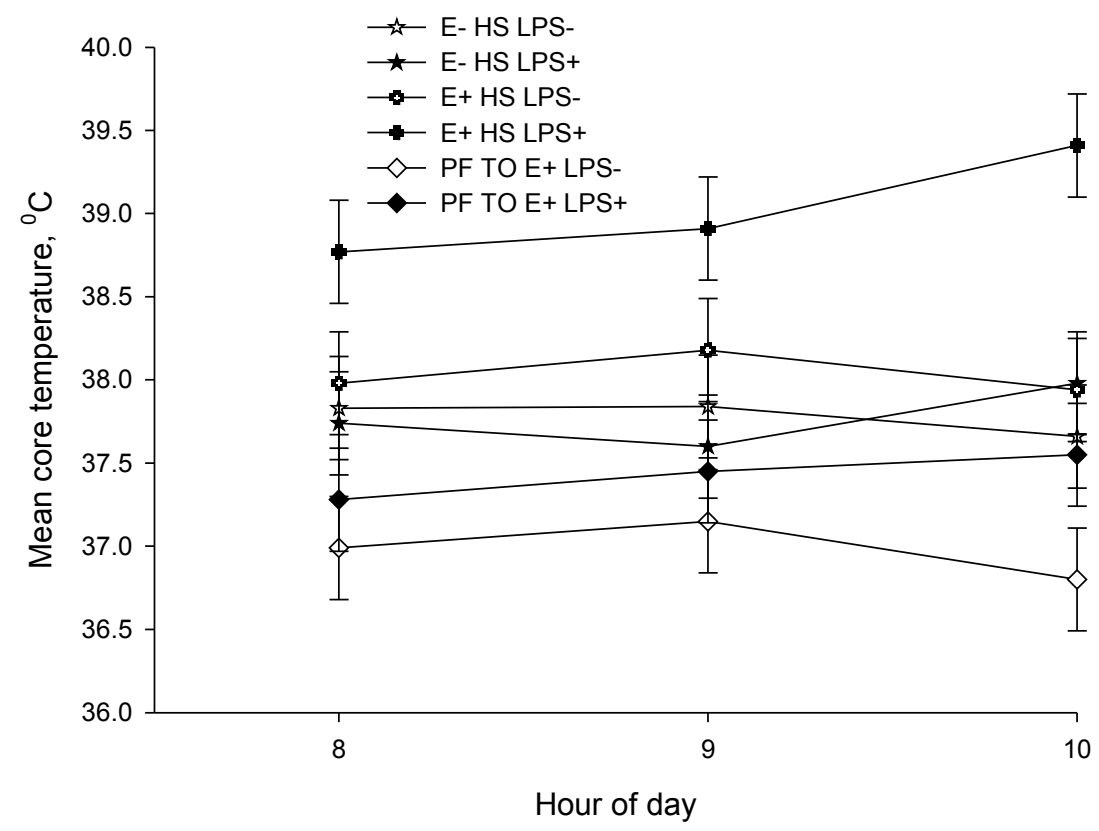

Figure 2.19. Change in average core body temperature of E-, E+ and PF TO E + in response to LPS challenge under heat stress conditions. The vertical lines above and below the first and last points indicate \pm SE. 


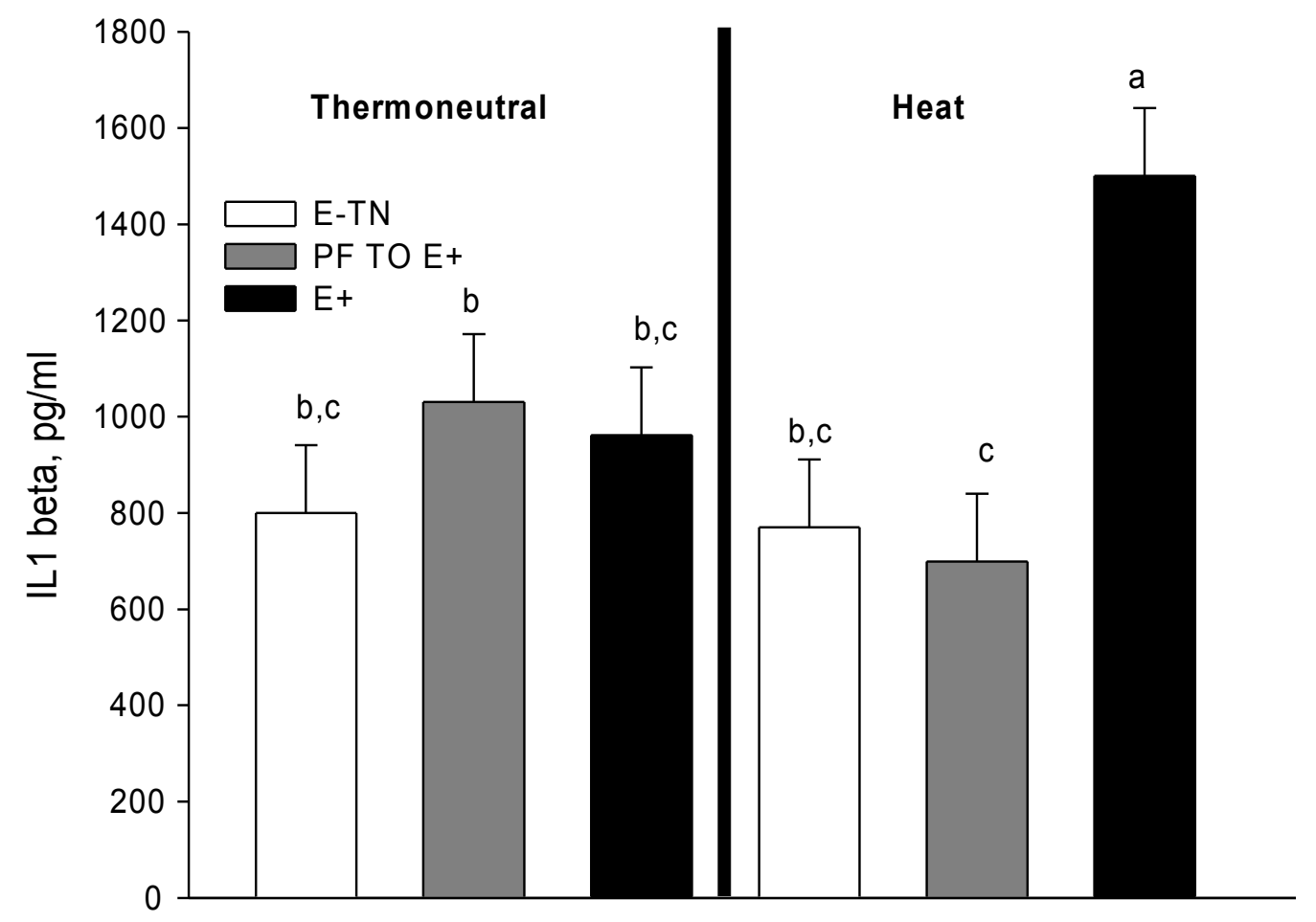

Figure 2.20. Horizontal bars represent levels of circulating IL1 $\beta$ in E-, E+, and PF TO E+ 2 hours after LPS challenge at thermoneutrality and under heat stress conditions. Different letters indicate significant differences across treatment groups. An endophyte effect $(\mathrm{P}>0.008)$ was observed, but a heat effect $(\mathrm{P}>0.61)$ was not seen. 


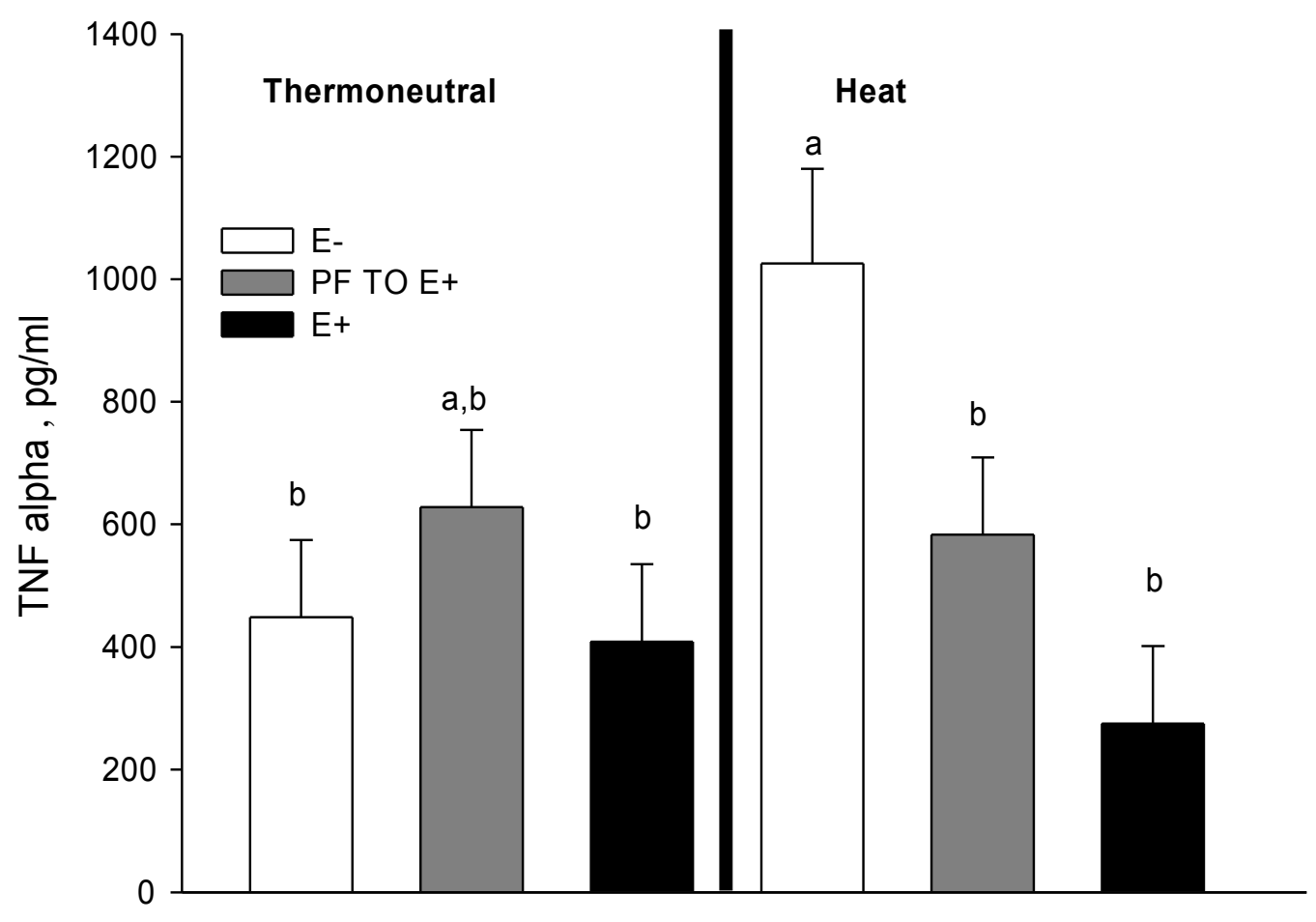

Figure 2.21. Horizontal bars represent levels of circulating TNF $\alpha$ in E-, E+ and PF TO E +2 hours after LPS challenge at thermoneutrality and under heat stress conditions. Different letters indicate significant differences across treatment groups. An endophyte effect $(\mathrm{P}>0.01)$ was observed, but a heat effect $(\mathrm{P}>0.21)$ was not seen. 


\section{Chapter IV}

\section{EFFECT OF SUBCHRONIC HEAT STRESS AND ERGOPEPTINE ALKALOIDS ON THE IMMUNE SYSTEM OF RATS}

\section{Abstract}

Research has shown that fescue toxicosis can impact immune function. A study was conducted to assess the combined impact of heat stress and ergopeptine alkaloids on the immune system. Telemetric temperature transmitters were implanted intraperitoneally in some animals to record core temperature (Tc). Feed inatke (FI) and and body weight (BW) were measured daily. Rats were fed diets containing ergopeptine alkaloids (E+; 32 ug Ergovaline/kg BW/d), no alkaloids (E-), or pair-fed (PF to E+ using an alkaloid-free diet). Each diet group was further divided into temperature treatments of thermoneutrality $\left(\mathrm{TN} ; 21^{\circ} \mathrm{C}\right)$ or heat stress $\left(\mathrm{HS} ; 33^{\circ} \mathrm{C}\right)$. Rats received diet treatments at TN for 1 week followed by 21 days in HS or TN environments. At the end of study, rats were given an intraperitoneal LPS challenge at dose rate of $100 \mu \mathrm{g} / \mathrm{kg} \mathrm{BW}$ and monitored for 2 hours. Blood samples were collected at the end of study and analyzed using flow cytometry for various lymphocyte percentages. Daily FI of E+ rats averaged $2 \mathrm{~g}$ below E- in both environments during the last treatment week $(\mathrm{P}<0.05)$. Moreover, $\mathrm{Tc}$ of $\mathrm{E}+$ rats was more than $0.9^{\circ} \mathrm{C}$ above E- level at the end of the treatment period $(\mathrm{P}<0.05)$. T cell $\%$ of total lymphocytes in E+ HS group was lower $(\mathrm{P}<0.05)$ compared to all the other heat stress and E-TN groups. Caloric restriction combined with heat stress reduced $\mathrm{CD} 4 \%$ and $\mathrm{CD} 8 \%$ percentage to half that of E-HS $(\mathrm{P}<0.05)$. In summary, we noted several significant effects of heat stress on the T cell compartment of the immune 
system. Comapred to short term the total T-lymphocyte, CD4+ and CD8+ percentages of E+HS and PF TO E+HS rats in this study were significantly suppressed. Some effects were independent of exposure to ergopeptide alkaloids, and none were a specific consequence of reductions in food intake associated with heat stress or fescue toxicosis.

\section{Introduction}

Tall fescue (Lolium arudinaceum) is commonly used in the Midwest United States as pasture grass. It has a symbiotic relationship with the fungus Neotyphodium coenophialum (Malinowski et al., 2005). This symbiotic relationship makes the plant very hardy and it thrives well in marginal, acidic and poorly drained soil. However, this relationship causes fescue toxicosis which results in an annual loss of over 600 million dollars in the United States (Hoveland, 1993). Ergopeptine alkaloids, cause a wide range of clinically non-specific symptoms such as reduced FI, body weight gain, activity, and thriftiness of the animal (Stickland et al., 1993). Other complications are fescue foot, summer slump, sloughing of peripheral tissues in ear and necrosis. Reduced prolactin level is a sensitive indicator of fescue toxicosis. Reduction in prolactin may also contribute to alterations in the immune system (Porter, 1995). The reproductive performance in affected in both male and female animals, embryonic development is also affected by the toxins (Collier et al., 2006). Cattle are mainly affected by the toxin (Cross et al., 2005; Oliver, 2005). However, horses (Cross et al., 1995), rabbits (Filipov et al., 1998), sheep (Gadberry et al., 2003), rats (Spiers et al., 2005), and mice (Varney et al., 1991) are also affected.

Effects of fescue toxicosis on the immune system had been studied using the mice and cattle model. Studies using cattle model, have shown that long term exposure to endophyte infected fescue doesnot compromise the animals ability to 
initiate production of pro-inflammatory cytokines like TNF $\alpha$ and IL6 (Saker et al., 1998; Filipov et al., 1999). Dew et al. (1997) showed that feeding mice with high levels of ergopeptine alkaloids, caused an increase in T-suppressor cells in thermoneutral conditions. This could lead to decrease in total T-lymphocyte percentages.

There is evidence to show that rats adapt to core body temperature, FI and body weight gain during long term exposure to fescue toxicosis (Settivari et al., 2006). Rats receiving $\mathrm{E}+$ and $\mathrm{E}$ - diet in the heat show adaptation to core body temperature. However, rats receiving E- diet adapted better than E- rats (Horowitz and Meiri, 1993; Matthew, 1997; Settivari et al., 2006). Upregulation of genes related to gluconeogenesis, lipid metabolism, protein catabolism and hepatic detoxification (CYP P450 isozymes) may also contribute to long term adapation to increased FI and body weight gain. There is very little information available on the adaptation to fescue toxin and effects on the long term effects of fescue toxicosison the immune system, therefore we decided to evaluate the long term effects of fescue toxin, heat stress and calorie restriction on the immune system. In this study we hypothesize that long term exposure at higher temperatures may allow rats to adapt to effects of heat stress and endophyte, but not the combination of both. 
The treatment diets, statistical analysis and sample experimental deisgn(except

21 days in heat) are all similar to short term studies. Here they are listed again.

\section{Materials and Methods}

\section{Animals and treatment diets}

Male Spargue Dawley rats $(\mathrm{n}=72 ; 350$ to $400 \mathrm{~g}$ BW) were implanted intraperitoneally, with telemetric transponders (Respironics, Bend, OR) to record core body temperature (Tc) and activity (Appendix 1). They were then were allowed a post operative recovery period of one week, and placed in to two chambers with similar conditions and ambient temperature. Dataloggers (HOBO Boxcar Prodata; Onset Computer Corp., Bourne, MA) were placed in each chamber to record ambient temperature and percent relative humidity throughout the study. Animals were maintained in 12:12 day light cycles, and percent relative humidity was maintained at $50 \pm 5 \%$, which was similar to previous studies in our lab (Spiers et al., 2005, Settivari et al., 2006). Feed and water were provided ad libitum throughtout the study period. Rats were fed either uninfected tall fescue seeds (E-) or infected tall fescue seed (E+; $32 \mu \mathrm{g} \mathrm{EV/kg} \mathrm{BW/d).} \mathrm{These} \mathrm{levels} \mathrm{were} \mathrm{estimated} \mathrm{by} \mathrm{standard} \mathrm{protocol}$ using HPLC (Rottinghaus et al., 1993). Composition of diet was similar to other studies in our lab (Spiers et al., 2005). All diets were stored at $4^{\circ} \mathrm{C}$ before feeding rats. FI (FI) and body weight were measured every day at 0900. Pair-fed (PF) diet was calculated by dividing the average FI of E + animals by the average body weight on that particular day. The net value was multiplied by the body weight of each PF animal to calculate the amount of feed, for that day

Rats were randomly divided into 6 treatment groups: E+ diet fed at thermoneutrality $(\mathrm{E}+\mathrm{TN}), \mathrm{E}-\operatorname{diet}$ fed at TN $(\mathrm{E}-\mathrm{TN}), \mathrm{E}-\operatorname{diet}$ pair-fed to $\mathrm{E}+\mathrm{TN}$ at TN 
(PF to E+TN), E+diet fed 7 days at TN followed by 21 days at HS, (E+HS), E- diet fed 7 days TN followed by 21 days at HS (E-HS), E- diet pair-fed to E+ HS at HS.(PF to E+ HS). Thermoneutral and heat stress groups were housed in separate chambers.

\section{Experimental design.}

Once FI and Tc were stabilized, baseline values for these parameters were collected for all animals in both rooms (HS and TN) for a period of five days. Following this period, rats in both chambers received their respective treatments (i.e., $\mathrm{E}+, \mathrm{E}-$, and $\mathrm{PF}$ to $\mathrm{E}+$ ) at $\mathrm{TN}$, for a period of one week. Following this period, ambient temperature of one of the rooms was increased to a constant temperature of $33^{\circ} \mathrm{C}$ for rest of the study and the other chamber was continuously maintained at $21^{\circ} \mathrm{C}$ (Figure. 3). On the twenty second day of this period at 0830 , half of the rats from each treatment groups $(\mathrm{n}=6)$ received an intraperitoneal LPS injection $(100 \mu \mathrm{g} / \mathrm{kg}$ BW) and other half $(n=6)$ received a control intraperitoneal injection of 2 mls sterile saline. After 2 hours, rats were anesthetized using xylazine $(13 \mathrm{mg} / \mathrm{ml})$ and ketamine $(87 \mathrm{mg} / \mathrm{ml})$, at dose rate of $0.1 \mathrm{mg} / \mathrm{kg} \mathrm{BW}$. Blood samples were collected using intracardiac puncture.

\section{Sample preparation}

Whole blood was collected in heparinized tubes and processed using the ficollhypaque centrifugation method for isolation of lymphocytes (Appendix. 2). All analyses were done at the University of Missouri hospital, flow cytometry core facility). Samples were counted by flow cytometery in the cell and immunobiology core facility, at the University of Missouri hospital using BD FASC calibur 
instrument. Serum was separated from whole blood for cytokine assays, using Quantikine IL-1 assays and Quantikine TNF $\alpha$ (R \& D systems).

\section{Statistical analysis}

All data were analyzed using Analysis of Variance ANOVA (JMP® 8; SAS Institute, Inc., Cary, NC). Repeated measures ANOVA of SAS (SAS Institute,,Inc, Cary, NC) was used to ascertain time-related changes in FI, body weight, core body temperature, motor activity, and levels of lymphocytes and cytokines. Standard least square model fit (JMP®, SAS Institute Inc, Cary, NC) was used for analysis of all parameters. Significance was reported based on the Tukey-Kramer multiple comparison test (Steele and Torrie, 1981). A P value of less than 0.05 was reported as significant. A linear plot analysis was performed using the mean weight of all rats in a treatment for a particular day.

\section{Results}

\section{Feed intake}

During the pretreatment period, there was no significant endophyte $(\mathrm{P}>0.12)$ or endophyte by day effects $(\mathrm{P}>0.86)$ on FI (Figure. 3.1).

\section{Diet treatment}

Rats showed a significant reduction in FI in response to endophyte $(\mathrm{P}<0.0001)$. This response was seen within 24 hours of exposure to the endophyte. A significant day effect and endophyte by day effect $(\mathrm{P}<0.0001)$ were also observed. The FI of 
$\mathrm{E}+$ and $\mathrm{PF}$ TO E+ rats stayed below $(\mathrm{P}<0.05)$ FI of E- rats during the first four days of diet treatment (Figure. 3.2). Partial recovery in FI was observed from Days 5 to 7, when FI was not significantly different from pretreatment levels. However, the FI of E+ rats never reached that of E- rats (Figure. 3.1).

\section{Heat stress and diet treatment}

The FI of E-TN, E+ TN and PF TO E+ TN groups were not significantly different from one another during this period. In contrast, there were pronounced heat stress effects, with endophyte, thermal, day, endophyte by day, and thermal by day differences $(\mathrm{P}>0.0001)$. Rats maintained at thermoneutrality had a higher FI $(\mathrm{P}<$ 0.05) compared to rats exposed to HS (Figure. 3.3). The E+ TN group stabilized their FI at Day 5, and then maintained this level until end of the study period. At the end of the study E+TN was slightly lower than E-TN, but this dfference was not statistically significant. During first 3 days of HS (Days 8 to 10), FI of all HS rats were lower (P $<0.05$ ) compared to rats at thermoneutrality. After the $4^{\text {th }}$ day of HS (Day 11), E- HS had a slight increase $(\mathrm{P}<0.05)$ in FI compared to its FI on the fisrt day of HS. Their FI was significantly $(\mathrm{P}<0.05)$ lower than E-TN until end of study, but it was not significantly different from $\mathrm{E}+\mathrm{TN}$ rats. Conversely, $\mathrm{E}+\mathrm{HS}$ had a lower $(\mathrm{P}<0.05) \mathrm{FI}$ until the last day of study.

Rats reduced their FI in response to heat stress, endophyte, and the combination of both. The E+ HS rats reduced their FI by $54 \%$ compared to E- HS rats which reduced their FI by $45 \%$ on the first HS day (Day 8 ) compared to Day 7 of diet treatment. The PF TO E+ group followed the same pattern of E+ HS rats, but the effects were seen the following day since FI was calculated based on FI of the E+ HS group. 
Rats maintained at thermoneutrality showed a pattern of adaptation to endophyte, with the FI of E+ TN rats increasing from Day 5 of diet treatment. This was fairly consistent throughoutt the study period. The PF TO E + TN rats followed a similar pattern, but the effects were observed a day later. In the long-term study, E+ HS rats $\left(33^{\circ} \mathrm{C}\right)$ did not show any significant signs of recovery. The E+ HS rats FI increased by a small magnitude from Day 11 (i.e., Day 4 of heat treatment). However, the FI of this group was consistently lower than other treatment groups. The E-HS rats showed signs of recovery after Day 11, when their FI stabilized. Although their FI did not consistently increase, there were no major reductions after Day 11.

\section{Body weight}

\section{Pretreatment period}

There was no endophyte $(\mathrm{P}>0.007)$ or endophyte by day effects $(\mathrm{P}>0.81)$ on body weight during this period. However, there were significant day effects $(\mathrm{P}<$ 0.0001). The day effect was attributed to the daily body weight gain observed in rats under normal conditions (Figure. 3.4).

\section{Diet treatment}

Diet treatment did not result in a significant difference between body weights of E-, E+, and PF TO E+ rats. Although there was no endophyte effect $(\mathrm{P}>0.21)$, there was both day $(\mathrm{P}<0.0001)$ and endophyte by day effects $(\mathrm{P}<0.0001)$. This shows that intake of an endophyte-infected fescue diet at thermoneutrality had a limited impact on growth. The E- rats were not affected and their body weight increased $(\mathrm{P}<$ $0.05)$ through out the treatment period. There was a decrease in body weight in 
response to the $\mathrm{E}+$ diet, and the response of animals on the $\mathrm{PF}$ to $\mathrm{E}+$ treatment followed a similar pattern (Figure. 3.5). The body weight of E+ rats were lower than E- rats during the diet treatment period. The E+ rats average daily weight gain was reduced compared to pretreatment levels until Day 5. Analysis using a linear plot showed a significant increase in body weight of E- TN (3.2g/d) compared to E+TN and E-TN rats which lost $1.46 \mathrm{~g} / \mathrm{d}$ and $0.86 \mathrm{~g} / \mathrm{d}$ respectively. These findings are similar to the short-term studies.

\section{Heat stress and diet treatment}

Exposure to TN conditions on the uninfected diet resulted in a continued increase $(\mathrm{P}<0.05)$ in BW until the last day of study. This increase began on Day 5. However, the E+ TN group never reached the level of the E-TN group. During the thermal treatment period, there were significant day, endophyte by day, and thermal by day effects. Body weight decreased $(\mathrm{P}<0.05)$ in response to $\mathrm{HS}\left(33^{\circ} \mathrm{C}\right)$. As stated before, the reduction in body weight gain occurs after the reduction in FI, and the pair-fed effects are seen a day after a change occurs for the E+ rats. Considering this, the body weight reduction below TN level of all HS groups occurred after Day 9 (Figure. 3.6). Both E- HS and E+ HS groups had lower body weights compared to pretreatment and diet treatment levels until day 10 of study. Then E-HS started to increase it body weight gain, but it stayed lower than $(\mathrm{P}<0.05) \mathrm{E}-\mathrm{TN}$ until last day of study. The E+ HS rats had lower body weights than E- HS until the last day of study (21 days in heat).

A regression line plot showed that the E-TN, E+TN, and PF TO E+TN gained 1.9 $\mathrm{g} / \mathrm{d}, 0.1 .3 \mathrm{~g} / \mathrm{d}$ and $1.07 \mathrm{~g} / \mathrm{d}$, respectively, from Days 8 to 28 . The body weight of rats at TN was significantly $(\mathrm{P}<0.05)$ higher than the $\mathrm{E}+\mathrm{HS}$ rats over this time period. A 
reduction in body weight of E+ HS (-1.25 g/d) and PF TO E+ HS (-0.17 g/d) was observed from the first day of HSuntil the end of study. Interestingly, E-HS gained weight at a slow pace of $0.79 \mathrm{~g} / \mathrm{d}$.

This study showed that $\mathrm{E}+$ treatment at $\mathrm{TN}$ can result in recovery from weight loss, but even with this recovery it may never reach the E- TN level. At the end of the study, there was no significant difference in the body weight of E+ TN and E-TN rats. The E- HS group showed signs of adaptation, by starting to gain weight on Day 10 . This was constant until the end of study. In contrast, E+HS rats did not show any trend towards weight gain until the end of the study (Day 21 in HS). This could be due to the effects of reduced FI.

\section{Core body temperature}

\section{Pretreament period}

The average core body temperature was not significantly different among treatment groups prior to treatment. Endophyte $(\mathrm{P}>0.58)$ and endophyte by day effects $(\mathrm{P}>00.47)$ were not seen. The average core body temperature varied from $37.4^{\circ} \mathrm{C}$ to $37.6^{\circ} \mathrm{C}$ among treatment groups (Figure. 3.7 ).

\section{Diet treatment}

In response to diet treatment, there were significant day $(\mathrm{P}<0.0001)$ and endophyte by day effects $(\mathrm{P}<0.0001)$. The groups that consumed the uninfected fescue diet did not show any significant variation in core body temperature, and it ranged from $37.4^{\circ} \mathrm{C}$ to $37.6^{\circ} \mathrm{C}$ (i.e., similar to pretreatment levels). Average daily core temperature of rats consuming the infected fescue was lower in response to 
endophyte. This trend towards hypothermia was minimal, and resulted in only a $0.1^{\circ} \mathrm{C}$ reduction which was not physiological relevant. On these days, the core body temperature of $\mathrm{E}+$ rats was lower than pretreatment $\mathrm{E}+$ core body temperatures (Figure. 3.8). During diet treatment, the PF TO E+ rats also had lower body temperatures compared to E- rats.

\section{Heat stress and diet treatment}

There were thermal, day, thermal by day, and endophyte by day effects $(\mathrm{P}<$ 0.0001) during HS period. The Tc of E- TN, E+TN and PF to E+ TN rats did not differ significantly. The E+TN and PF TO E + TN rats were slightly lower than E-TN rats, but this difference was not statistically significant. In contrast, the E- HS, E+ HS, and PF TO E+ HS rats had a dramatic increase $(\mathrm{P}<0.05)$ in Tc on the first HS day (Day 8) compared to TN rats. The E+ HS group had the greatest Tc increase $(\mathrm{P}<$ 0.05) (i.e., $0.98^{\circ} \mathrm{C}$ above Day 7 level at $\mathrm{TN}$ ) compared all other treatment groups, including E-HS, which increased $0.65^{\circ} \mathrm{C}$ above Day $7(\mathrm{P}<0.05)$, and $\mathrm{PF}$ TO E+HS groups in the first day of HS. (Figure. 3.9). Both E-HS and PF TO E+ rats showed signs of adaptation after three HS days, when Tc was reduced and stabilized at a level lower than that of E+HS. The Tc of PF rats was lower compared to E- HS rats. The $\mathrm{E}+\mathrm{HS}$ rats showed no signs of adaptation at $33^{\circ} \mathrm{C}$, with $\mathrm{Tc}$ on the last day of study being similar to that of first HS day (Figure. 3.10). During the entire HS period, Tc of $\mathrm{E}+\mathrm{HS}$ rats ranged from 38.2 to $38.9^{\circ} \mathrm{C}$ compared to E- HS which ranged from 37.9 to $38.3^{\circ} \mathrm{C}$. This suggests that $\mathrm{E}+\mathrm{HS}$ rats may never be able to adapt to the combined effects of this level of HS and intake of ergopeptine alkaloids found in endophyteinfected fescue. 


\section{Circadian changes in core body temperature}

\section{Pretreatment}

Both an endophyte effect $(\mathrm{P}<0.58)$ and endophyte by hour $(\mathrm{P}<0.12)$ interaction were absent during the pretreatment period. The highest Tc was observed during 2100 to 0200 and lowest was recorded around 0800 to 0900 . There was a decrease in Tc $(\mathrm{P}<0.05)$ from 0600 to 0700 , before lights were turned on, and an increase from 1700 to 1800 before lights were turned off. The greater Tc increase $(\mathrm{P}<0.05)$ from evening level was observed after 2100. In addition, there was a spike in Tc in response to daily feeding.

\section{Diet treatment}

All treatment groups exhibited circadian changes in TC. Diet treatment showed significant endophyte by hour $(\mathrm{P}<0.0001)$ and thermal by hour effects $(\mathrm{P}<0.0011)$. Both the E+ and PF TO E+ groups had a lower Tc $(\mathrm{P}<0.05)$ from 0700 to 1000 compared to 2100 to 0200 (Figure. 3.11). Likewise, their Tc stayed lower during the day from 1100-1700, when the lights were turned on. However, their Tc increased after lights were turned off and it did not differ from E- treatment groups. This suggests that TN hypothermia in $\mathrm{E}+$ rats might be best documented during the day when lights are turned on. A daily increase in Tc was documented in response to feeding, and the PF rats had the greatest increase in Tc as they consume feed immediately.

\section{Heat stress and diet treatment}


Significant diet, thermal, hour, diet by hour, and thermal by hour effects $(\mathrm{P}<$ $0.0001)$ were observed in this phase of the experiment. Heat stress increased $\mathrm{Tc}(\mathrm{P}<$ 0.05) of rats, and all HS groups followed the same circadian pattern as TN rats. The only appreciable difference was the absence of a pronounced Tc increase after feeding in E-HS and $\mathrm{E}+\mathrm{HS}$ rats. Greater $(\mathrm{P}<0.05)$ differences in Tc between HS and TN rats were observed from 2100 to 0500 when rats were most active (Figure. 3.12). This suggests that the difference between $\mathrm{HS}$ and $\mathrm{TN}$ rats is greatest at night when the rat Tc increases (Figure. 3.12).

\section{General gross motor activity}

\section{Pretreatment}

Gross motor activity did not differ significantly among treatment groups during the pre-treatment period. No endophyte $(\mathrm{P}>0.84)$ or endophyte by day effects $(\mathrm{P}>$ 0.12) were observed.

\section{Diet treatment}

There was no endophyte $(\mathrm{P}>0.94)$ or endophyte by day effects $(\mathrm{P}>0.39)$ on average daily activity. A non-significant decrease in activity was observed in E+ rats after diet treatment (Figure. 3.13).

\section{Heat stress and diet treatment}

Significant day $(\mathrm{P}<0.0001)$ and thermal by day effects $(\mathrm{P}<0.0001)$ were observed in response to $\mathrm{HS}$. The E-TN, E+TN, and PF TO E+TN rats daily activity did not differ significantly. All HS rats had a slightly higher activity compared to TN 
rats on the first 2 days of HS (Days 8 and 9), and then reduced their gross motor activity. Both E-HS and PF TO E+HS groups had the greatest activities on Days 8 and 9. A marked reduction in activity was seen in E+HS rats during the last two weeks of HS (Figure. 3.13). This reduction was significantly $(\mathrm{P}<0.05)$ lower than first 2 HS days. Reduced activity could be an indication of sickness behaviour in these rats.

\section{Circadian changes in activity}

\section{Pretreatment}

A set pattern of activity was observed during the entire study. Circadian rhythm of activity was similar to that of Tc. The greatest decrease in activity was seen from 0600 to $0700(\mathrm{P}<0.05)$ before lights were turned on, and the increase in activity was seen from 1700 to 1800 before lights were turned off. A greater increase in activity was observed from 1800 to 1900 . The lowest activity was observed at 0800 . A significant increase in activity was observed in all treatment groups at 1000 corresponding to the daily feeding time. A diet effect $(\mathrm{P}>0.57)$ was not observed during this period.

\section{Diet treatment}

During this period, the circadian rhythm for activity was similar to pretreatment levels and did not change significantly (Figure. 3.14). There were hour, endophyte by hour, and thermal by hour effects $(\mathrm{P}<0.0001)$. However, there was no endophyte only significant effect on the circadian pattern of activity $(\mathrm{P}>0.35)$. 


\section{Heat stress and diet treatment}

There were significant hour, endophyte by hour, and thermal by hour effects $(\mathrm{P}<0.0001)$ in response to thermal stress. The TN rats followed a similar pattern observed during diet treatment. The E+ HS rats had lower activity compared to the ETN rats from 1900 to 2100 . The PF TO E+HS rats demonstrated a greatest $(\mathrm{P}<0.05)$ increase in activity during daily feeding time (Figure. 3.15).

\section{Lymphocytes}

\section{Natural killer cells}

Natural killer cells are cells of the innate immune response that act against intracellular virus and tumor cells. The E-TN, PF TO E+TN, and E+TN groups in the present study did not differ significantly. However, a thermal effect was observed (P $<0.0024)$. All HS rats had a higher NK cell percentage compared to the same treatment under thermoneutral conditions. The PF TO E+ HS group showed a marked increase $(\mathrm{P}<0.05)$ in NK Cell percentage compared to all TN groups (Figure. 3.16). The E+ HS and E- HS groups had greater values than their respective TN groups. This shows that stress caused by caloric restriction, HS, and combination of endophyte and HS increases NK cell percentages to anticipate infections.

\section{B-lymphocytes}

The B-lymphocytes are cells that help in antibody production. Diet $(\mathrm{P}<0.16)$ and thermal effects $(\mathrm{P}<0.14)$ were not observed. The B-lymphocyte percentages in the PF TO E + HS and E+HS groups were lower compared to the other treatment groups. However, this reduction was not statistically significant. 


\section{T-lymphocytes}

Total T- lymphocyte percentages varied in response to the endophyte diet and heat treatment. The E-TN, E+TN, and PF TO E+ TN treatments did not differ significantly. However, the T-lymphocyte percentage of the E+ HS group was lower $(\mathrm{P}<0.05)$ than for E-TN, E+TN, and PF TO E+ HS groups (Figure. 3.17). The E- HS and PF TO E+ HS groups did not differ significantly from their TN counterparts.

\section{CD4+ cells}

The CD4+ cells are a subclass of T-lymphocytes. They are commonly referred as helper-T cells. The E-TN, E+TN, and PF TO E+ TN treatment groups did not differ significantly from each other. The CD4+ percentage in the PF TO E+ HS group was the lowest, and it was significantly lower than $(\mathrm{P}<0.05)$ the $\mathrm{E}+\mathrm{TN}$, PF TO $\mathrm{E}+\mathrm{TN}$, and E-HS groups (Figure. 3.18). The E+ TN and E+ HS groups did not vary significantly from one another. However, there was a marked decrease in the E+HS treatment group.

\section{CD8+ cells}

The CD8+ cells are a subclass of T-lymphocytes. These cells are also called as cytotoxic T cells. The E-TN, E+TN, and PF TO E+ TN treatment groups did not differ significantly from one another (Figure. 3.19). The CD4+ percentage in the PF TO E+ HS group was lowest, and it was significantly lower than $(\mathrm{P}<0.05)$ all other treatment groups. Other HS groups did not show significant changes.

\section{LPS administration}




\section{LPS at thermoneutrality}

Core body temperatures of all LPS-challenged rats did not differ significantly across treatment groups. Both hour $(\mathrm{P}<0.0217)$ and thermal by hour effects $(\mathrm{P}<$ 0.0126) were observed. However, there were no LPS $(\mathrm{P}>0.19)$ and LPS by hour (P $>0.09$ ) effects. The Tc of E- TN LPS rats showed a marginal decrease of $0.5^{\circ} \mathrm{C}$ from 0800 to 1000 (Figure. 2.20), following a natural circadian pattern. The E- TN LPS+ rats had a non-significant decrease of Tc $0.7^{\circ} \mathrm{C}$ at 1000 (i.e., 2 hours after LPS challenge). The PF TO E+ TN LPS+ rats showed a nonsignificant decrease at 1000. The E+TN LPS+ rats also had a Tc decrease of $0.6^{\circ} \mathrm{C}$ from its level at 0800 . A trend towards hypothermia was noted under TN conditions for all LPS challenged groups (Figure 3.20).

Activity followed the same trend as Tc, with all groups having a lower activity at 1000. However, the LPS-challenged rats exhibited a greater decrease in activity compared to the LPS-control rats. Both hour and thermal effects were observed $(\mathrm{P}<$ 0.0001). However, there were no LPS $(\mathrm{P}>0.24)$ or LPS by hour effects $(\mathrm{P}>0.14)$.

\section{LPS at heat stress}

In the HS environment, there was the same pattern on an initial Tc spike from 0800 to 0900 for all treatment groups. Both hour $(\mathrm{P}<0.0217)$ and thermal effects $(\mathrm{P}$ $<0.0126)$ were observed. However, there were no LPS $(\mathrm{P}>0.19)$ or LPS by hour ( $>0.09$ ) effects. Similar to the TN response, the LPS control rats decreased Tc from 0800 to 0100 . Both E+ HS LPS+ and E-HS LPS+ groups increased their Tc slightly from 0900-1000, but it was not statistically significant. This suggests that there is a trend towards hyperthermia in HS rats (Figure. 3.21). 
There was a general trend toward an increase in activity, followed by a decrease under HS conditions. The E+HS LPS+ rats had the lowest activity at 1000, but this decrease was not significant. All other rats also had a lower activity at 1000 following their natural circadian pattern.

\section{IL1及}

Rats maintained under TN and HS conditions responded with an increase in circulating levels of IL $1 \beta$ when $100 \mu \mathrm{g} / \mathrm{kg}$ BW of LPS was administered intraperitoneally. Two hours after LPS challenge all treatment groups that received the LPS injection had higher $(\mathrm{P}<0.05)$ levels of circulating IL1 $\beta$ compared to the LPS control groups (Figure. 3.22). The values for E- TN, E+TN, and PF TO E+ TN rats did not vary significantly from one another. In addition, a significant thermal effect $(\mathrm{P}<0.0331)$ was observed. The IL1 $\beta$ values for all HS treatments were higher than their respective TN groups. The IL1 $\beta$ value for PF TO E+ HS rats was significantly $(\mathrm{P}<0.05)$ higher than for E-TN and PF TO E+ TN groups. There was not significant different between E-HS and E+HS rats.

\section{TNFa}

Rats that received LPS challenge responded with an increase in serum TNF $\alpha$ level. In contrast, control rats had below detectable levels of TNF $\alpha$. Both significant endophyte $(\mathrm{P}<0.0300)$ and thermal effects $(\mathrm{P}<0.0181)$ were noted. The E- TN, E+TN, and PF TO E+ TN rats were not significantly different from one another (Figure. 3.23). Similar to IL1 $\beta$, all HS groups had higher circulating levels of TNF $\alpha$ compared to the same TN groups. The E+ HS group was significantly higher $(\mathrm{P}<$ 
0.05) than all TN treatment groups and the PF TO E+ HS group. Pair-fed groups had the lowest circulating levels of TNF $\alpha$ under TN and HS conditions.

\section{Discussion}

\section{Feed intake}

In this final trial, FI was reduced by $40 \%$ on the first day of diet treatment. This is in agreement with other studies which have reported more than a $50 \%$ reduction in FI on the first day of diet treatment at thermoneutrality (Roberts et al., 2002; Spiers et al., 2005; Settivari et al., 2008). FI of rats in the present study showed a partial recovery on Day 4. Again, this is consistent with past studies were a recover in FI was observed on day 4 of exposure to the E+ diet (Robert et al.,2002; Spiers et al., 2005).

The present study showed that both heat stress and intake of endophyte-infected tall fescue independently reduces FI. In addition, the combination of both results in an even greater reduction. These results are consistent with previous reports from this laboratory (Spiers et al., 2005; Al-Tamimi et al., 2007). The E+ HS rats reduced their

FI by $63 \%$ compared to E- HS rats which reduced their FI by $50 \%$ on the first day of heat stress (Settivari et al., 2006). Reduction in FI in response to heat stress has been reported previously (Johnson and Cabanac, 1982; Johnson and Stack, 1989 and DeVries et al., 1993). Reduced FI may be due to decreased duration, frequency and quantity of FI (Johnson and Cabanac, 1982; Johnson and Stack, 1989). Al-Tamimi et al. (2007) stated that a decreased FI is an adaptive mechanism to lower post prandial heat production.

The E+HS had a lower feedintake until the last day of study and never reached the levels of E-HS rats. This is in agreement inprevious reports (Settivari et al., 
2006). Similar, decreases in FI of E+ HS rats above the levels of E-HS has been reported in the past in cattle (Aldrich et al., 1993) and sheep (Gadberry et al., 2003). These findings show that heat stress may negate the adaptive response in rats to E+ diet. Reduction in FI for the present study was higher than the values of previous reports. This reduction is attributed to higher thermal stress $\left(33^{\circ} \mathrm{C}\right)$, compared to past studies. Long-term studies (Spiers et al., 2005; Settivari et al., 2006) showed that E+TN and E-HS may recover partially from their reduced FI. However, their FI never fully returns to the level of the control E- TN rat. There are several theories proposed stating various reasons for the reduction in FI. Strickland et al. (1993) related effects of fescue toxin and dopamine activation. Dopamine receptor activation may cause satiety, leading to decreased FI. Other studies have shown that serotonin receptors could be activated causing satiety in rats (Oliver, 2005). Al- Tamimi et al. (2007) stated that toxins associated with endophyte-infected fescue impacts 5-HT receptors causing a reduction in FI. There is consensus that hunger and FI are goverened by different factors, therefore fescue toxicosis could also act through more than one mechanism. Likewise, Spiers et al. (2005) ruled out the possibility that reduced FI is due to palatability of the diet. Reduced FI is seen as a protective mechanism to avoid postprandial metabolic heat production. This possibly gives the animal the ability to cope with increased core body temperature.

\section{Body weight}

Body weight gain in the present study was altered in response to change in FI as a result of heat stress or intake of ergopeptine alkaloids associated with endophyteinfected tall fescue. Body weight was reduced 24 hours following intake of the endophyte-infected diet, but this was not significant. Rats continued to lose body 
weight until Day 5 on the diet, with the first increase on Day 6. Similar, adaptation to body weight gain has been observed previously from day 4 to 6 (Spiers et al., 2005, Settivari et al., 2006).

It is interesting to note that at thermoneutrality the body weight of the $\mathrm{E}+$ and $\mathrm{PF}$ TO E+ rats were very similar. This is evidence to suggest that the effect of ergopeptine alkaloids on body weight gain is no more than the effect on FI. Farm animals that consume endophyte-infected tall fescue follow a similar pattern of body weight gain (Osborn et al., 1992; Gadberry et al., 2003). In the present study, rats fed the E+ diet started gaining weight from Day 6 and this continued until the last day of study.

Heat stress decreased average daily gain in E-, E+, and PF TO E+ treatment groups. The impact of heat stress on body weight gain was greater than the effect of endophyte alone. These results were consistent with previous results from our lab (Spiers et al., 2005; Al-Tamimi et al., 2007). In contrast to E+TN, E+ HS did not show a continuous pattern of increased body weight gain until the last day of study. Previous research reports a $63 \%$ decrease in average daily gain of $\mathrm{E}+$ rats compared to E- rats at thermoneutrality after 24 hours of exposure (Neal and Schmidt., 1985). The greatest difference in body weight of E+ and E- rats has been noted on Day 3 of treatment (Spiers et al., 2005).

Control rats in the heat also reduced the average daily body weight and this is in agreement with previous studies by other research groups (Johnson and Cabanac., 1982; De Vries et al., 1993). Decreased FI could have contributed to decreased daily weight gain in E-HS rats. Decreased metabolic heat production in summer is achieved due to decreased caloric intake and lowered thyroid hormone production, which helps heat stressed animals adapt to hyperthermia (Baccari et al., 1983). 
Recent genomic analysis has shown that genes related to protein catabolism and gluconeogenesis are upregulated at thermoneutrality for rats on the $\mathrm{E}+$ diet in thermoneutral conditions (Settivari et al., 2006). However, long term E+HS produced down regulation of genes related carbohydrate (transmembrane protein 123, 6phosphofructo-2-kinase/fructose-2,6-biphosphatase 2 and solute carrier family 37 member 4), lipid (acetyl-coenzyme a acyltransferase 2, acyl-coenzyme a oxidase and protein (eukaryotic translation elongation factor 2, and heterogeneous nuclear ribonucleoprotein 3) metabolism (Settivari et al., 2006) More confounding is the fact that even if rats started recovering from reduced FI, the effect of ergopeptine alkaloids on the mitochondrial genes relating to ATP production may prevent them from gaining weight (Settivari et al.,2006). This suggests that endophyte may impact bodyweight gain by more than one avenue. However, these effects are not significant unless combined with heat stress.

\section{Average core body temperature}

Average daily core temperature in the present study was lower for rats fed the endophyte-infected fescue diet at thermoneutrality. Likewise, the pair-fed rats at thermoneutrality had a lower core body temperature compared to E- rats. These findings are consistent with previous reports (Spiers et al., 2005; Settivari et al., 2006). Hypothermia in response to ergovaline at thermoneutrality has also been documented previously (Zhang et al., 1994; Spiers et al., 1995). The fact that pairfeeding, which causes caloric restriction, also leads to a lower core body temperature (Yoda et al., 2000) shows that reduced caloric intake could be a possible reason for the hypothermia seen in E+ rats at thermoneutrality. Although the core body temperature of $\mathrm{E}+$ rats at thermoneutrality was significantly lower than the 
pretreatment $\mathrm{E}+$ level it was not significantly different across treatment groups during diet treatment. These findings suggest than the $\mathrm{E}+$ diet by itself may not cause a harmful shift in core body temperature at thermoneutrality.

Exposure to heat stress $\left(33^{\circ} \mathrm{C}\right)$ in the present study significantly increased core body temperatures of E- HS, E+ HS, and PF TO E+ HS rats compared to the same treatment groups at thermoneutrality. Likewise, the E+HS group was significantly higher than either the E- HS or PF TO E+ HS rats for the duration of heat exposure. These findings are partially in agreement with past studies at $31^{\circ} \mathrm{C}$ (Spiers et al., 1995; 2005). The E+ HS rats in the current study did not show any signs of lowering their core body temperature for the duration of the study. Any reduction observed was only transient, and was followed by an increase in core body temperature. This is different from lower heat stress levels where a certain degree of adaptation was seen in the E+ HS rats (Settivari et al., 2006). Oliver, (2005) reported that binding of $\alpha$ adrenergic receptors, hampers vasodilation and heat loss mechanism in rats. This might explain why the E+HS could never reduce their Tc as they were continuously exposed to the the toxin. Al-Tamimi et al. (2007) showed that inihition nitric oxide could also prevent vasodilation leading to hyperthermia, which a common symptom fescue toxicosis.

Previous experiments have also shown a heat adaptive response for core body temperature. Rats exposed to $32^{\circ} \mathrm{C}$ to $33^{\circ} \mathrm{C}$ for 2 weeks, showed adaptation from Day3 and then their Tc began to decline (Horowitz and Meiri, 1993; Matthew, 1997). During heat stress, splanchnic and peripheral vasodilation (Horowitz, 1998) aid in heat loss from the body. Similarly, evaporative cooling, extending limbs to increase surface area, and spreading saliva are other ways to reduce core body temperature (Schmidek et al., 1983; Hubbard et al., 1982; Gordon, 1993). 


\section{General activity}

General activity was highly variable among treatment groups in the present study. No significant difference was observed across different treatment groups during pretreatment and diet treatment periods. These findings are in agreement with previous reports by Spiers et al. (2005). In the present study, heat stress reduced activity of rats. The PF TO E+ HS group had the highest activity during heat stress. We hypothesized that the relatively small quantity of feed given to these rats kept them extremely hungry, and this hunger could have driven them to explore for more food. The E+HS group had lowest activity at the end of study. This could be an indication of sickness behaviour as suggested by Spiers et al. (2005) due to the fescue toxicosis. Similar to our short-term studies, increased activity was observed in E-HS for the first two days. But this gradually reduced and at the end of the study this activity was considerably lower than other treatment groups. Al-Tamimi et al. (2007) also observed reduced activity in response to heat stress in long-term studies.

\section{Lymphocytes}

\section{Natural killer cells}

Natural Killer cells play a major role in innate antiviral defense (Biron, 1997). In the short term study the highest percentage was observed in E+HS rats, the paired-fed rats also had an increase in NK cell percentages. In the current study there was a marked increase in the NK cell percentage of PF TO E+ HS rats compared to other heat stressed and thermoneutral groups, suggesting that a higher magnitude of caloric restriction can trigger an increase in NK percentages and activity (Shen et al., 2009). 
It is important to note that a significantly lower CD8+ percentage in the PF TO E+ HS group, may have maintained NK cell percentages at a higher level. There is an inverse relationship between MHC 1(which interacts with CD8+) and NK cell percentages (Biron, 1997). If one is increased it decreases the other, therefore we suggest that lower levels of CD8+ (which interacts with MHC I) in PF TO E+ HS r ats may have contributed to the increase in NK cell percentages (Abbas et al., 2007). The same effects were not observed in PF TO E+ TN rats, as the magnitude of caloric restriction was very low compared to $\mathrm{PF}$ TO E+ HS rats. It was interesting to note that NK cell percentage was higher in all heat stressed groups compared to thermoneutral treatments. This suggests that heat stress may also contribute to increased NK cell percentages (Biron, 1997).

\section{B-lymphocytes}

Lymphocyte percentages across treatment in the present study were fairly constant, except for a reduction in the pair-fed treatment groups. In the current longterm study, B cell percentages were lower in the pair-fed treatment group exposed to heat stress compared to thermoneutral groups. Past studies have shown that both short- and long-term heat stress and intake of fescue toxin do not affect an animal's ability to mount an inflammatory or antibody response in response to pathogens (Rice et al., 1997; Filipov at al., 1999b). Our results are in agreement with these reports. Compared to our short-term study which showed a lower percentage in E+HS rats B cell percentages of E+ HS rats recovered in the long-term. An earlier report also noted that B-lymphocyte percentages improve 4 to 7 days after exposure to acute stress heat stress (Dracott and Smith, 1979). Heat stress and endophyte individually did not affect B cell percentages in the present study. This suggests that this antibody 
response may not be affected by individual exposure to heat stress or feeding animals an endophyte-infected diet at thermoneutrality (Rice et al., 1997; Filipov et al., 1999b).

\section{T-lymphocytes}

$\mathrm{T}$ cell percentage varied across treatment groups in the present study, with the $\mathrm{E}+$ group having the lowest percentage of $\mathrm{T}$ cells after long-term heat stress. The E+ TN was only slightly lower than the level found in E- TN rats. Settivari et al. (2006) reported a down regulation in TCR beta of rats exposed to $\mathrm{E}+$ diet in thermoneutrality. Earlier reports have stated that feeding mice an E+ diet increased T-suppressor cell percentage at the end of 43 days (Dew et al., 1990). However, they did not report the total T-lymphocyte percentages. Compared to our short-term studies, the E+ HS rats had lower T-lymphocyte percentages. It is possible that the activation of T-suppressor cells could have reduced the percentage of total Tlymphocytes. All treatment groups in the current study, except E+ HS treatment group, stabilized within a normal range for T-lymphocytes. This indicates that T-cell dependent, cell-mediated immunity could be compromised in these animals. The cellmediated immunity of E+HS could have been compromised more than the other groups due to the lower levels of T-lymphoctes and CD8+ cells. This reduction might make these animals susceptible to intracellular and extracellular pathogens.

\section{CD4+ and CD8+ cells}

Both CD4+ and CD 8+ percentages have been used in diagnosis of immunosuppressive conditions like HIV. (Taylor et al., 1989; Burcham et al., 1991) Many differences in these areas were observed across treatment groups in our long- 
term study. Several studies have reported that these percentages can change in chronic immunosuppressive diseases, and estimating total percentages are a better prognostic indicator than total cell count (Taylor et al., 1989; Burcham et al., 1991; An der Ryst et al., 1998). The CD4+ percentages were the lowest for the PF TO E+ HS rats. This suggests that caloric restriction in the heat may impact the T-cell -mediated response in rats. Ana et al. (2004) reported an increase in difference of $\mathrm{CD} 4+\mathrm{CD} 8+$ rats when subjected to caloric restriction. Identical to our findings, others using the mice model have reported a significant depletion of CD4+ and CD8+ cells in response to caloric restriction (Poetschke et al., 2000). These effects were observed at more than $40 \%$ caloric restriction at thermoneutrality. Conversely, PF TO $\mathrm{E}+\mathrm{TN}$ rats in the current long-term study received less caloric restriction, and therefore changes in the PF TO E+ group were not seen. Alterations in $\mathrm{CD} 4+$ and $\mathrm{CD} 8+$ percentages may compromise the rats ability to mount an immune response against both intracellular and extracelluar pathogens.

The E+ HS rats in the present study also had a marked reduction in CD8+ cells, in contrast to the E- HS rats which had a higher percentage. These changes suggest that intake of the $\mathrm{E}+$ diet in the heat may compromise the animals ability to mount an adequate cell-mediated response to intracellular pathogens, which are mainly counteracted by CD8+ cells. Most of the changes observed are in the T-cell lineages. Therefore, we propose that long-term caloric restriction and intake of the $\mathrm{E}+$ diet in the heat may compromise the long-term adaptive, cell-mediated immune response.

\section{Cytokine response to LPS}

Although core body temperatures did not differ significantly in response to LPS injection in the present study, there was a significant cytokine response. All treatment 
groups, including the rats that received endophyte diet, retained the ability to produce pro-inflammatory cytokines in response to LPS. This is in agreement with other studies (Rice at el., 1997; Filipov et al., 1999b). The E+ HS group showed the highest TNF $\alpha$ response, which might be associated with the increased amount of stress experienced by these animals. Increased pro-inflammatory response to endotoxin at thermoneutrality has been reported in mice and cattle fed E+ diet (Rice at el., 1997; Filipov et al., 1999a). Studies have shown that caloric restriction can reduce the proinflammatory response (Muthukumar et al., 2000). These studies examined TNF $\alpha$ level in pair-fed animals in thermoneutrality and showed a decreased activation in pairfed animals. The IL1 $\beta$ and TNF $\alpha$ levels in the E-HS group were marginally higher than for the E-TN group, suggesting that heat stress can increase the proinflammatory response in rats. Similar results have been reported in the past using the rat model (Bouchama et al., 1991; Lin et al., 1997). The marked increase noted in $\mathrm{TNF} \alpha$ level of $\mathrm{E}+\mathrm{HS}$ rats and the moderate increase in $\mathrm{E}+\mathrm{TN}$ rats revealed that these animals did not lose their ability to mount a proinflammatory response even after consuming an endophyte-infected diet for many days. This is consistent with a previous report using mice (Filipov et al., 1999a). However, it is important to note that an increased response may also be harmful to tissue and cell functions. In the long term study rats had a higher level of TNF $\alpha$ compared to all other treatment groups, this might cause an increased inflammatory condition in the body, predisposing the animal to shock and sepsis 


\begin{tabular}{|c|c|c|c|c|}
\hline PERIODS & $\begin{array}{l}\text { TN } \\
\text { Baseline }\end{array}$ & Diet Trt & $\begin{array}{l}\text { Diet + } \\
\text { TaTrt }\end{array}$ & LPS \\
\hline DAYS & -5 to -1 & 1 to 7 & 8 to 28 & 29 \\
\hline Ta & $21^{\circ} \mathrm{C}$ & $21^{\circ} \mathrm{C}$ & $21^{\circ} \mathrm{C} / 33^{\circ} \mathrm{C}$ & \\
\hline
\end{tabular}

Figure 3. Timeline of long term study. Time line of study shows E+ , E- and PF TO E+ rats maintained in pretreatment, diet treatment and thermal treatment. The timeline also shows the number of days that rats were placed on each of these stressors and the temperature of chambers during the specific treatment periods. 


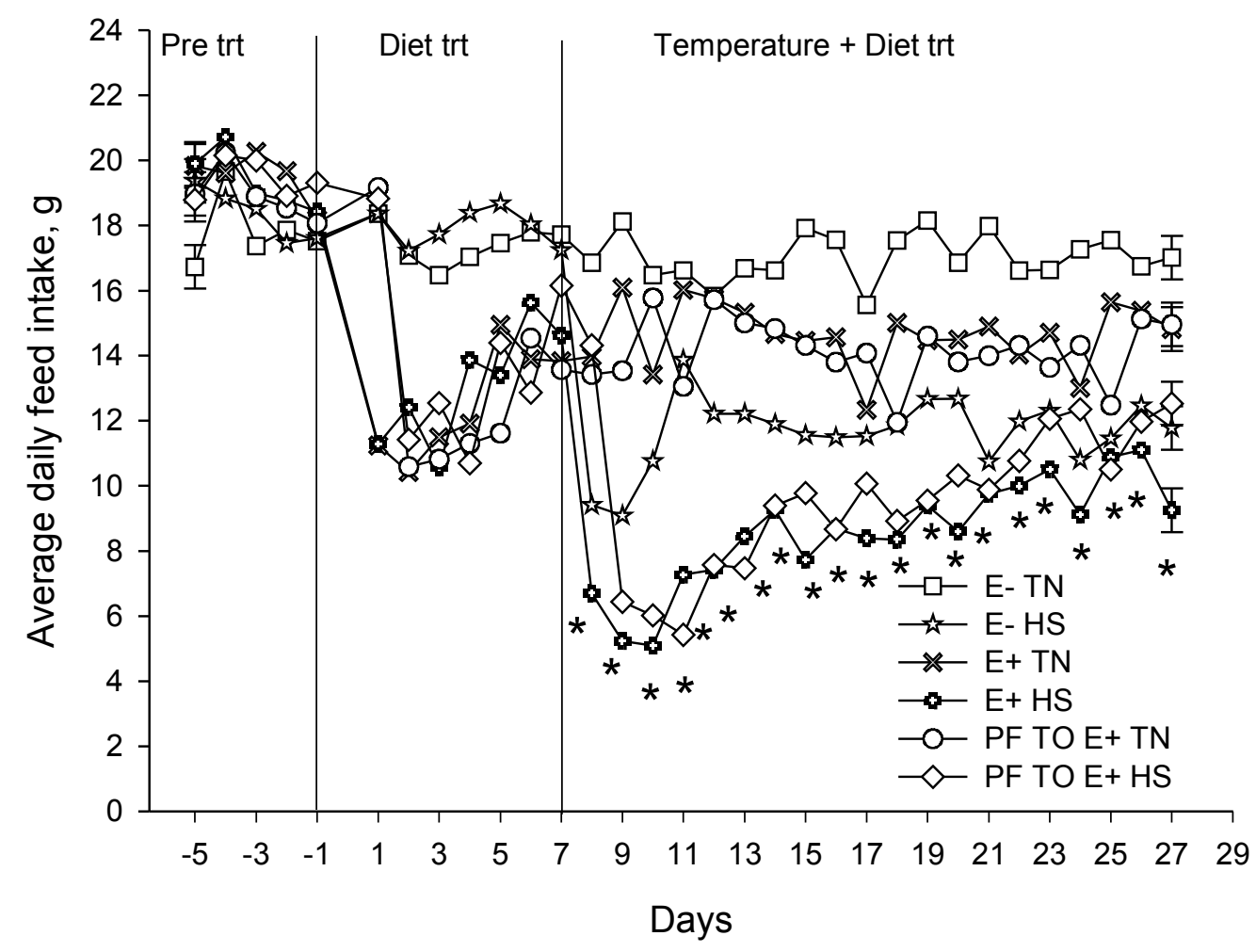

Figure 3.1. Average daily feed intake of E-, E+, and PF TO E+ rats under HS and TN conditions. An asterisk (*) shows the statistical significance between E+ HS and $\mathrm{E}-\mathrm{TN}$ groups. The vertical lines above and below the first and last points indicate \pm SE. 


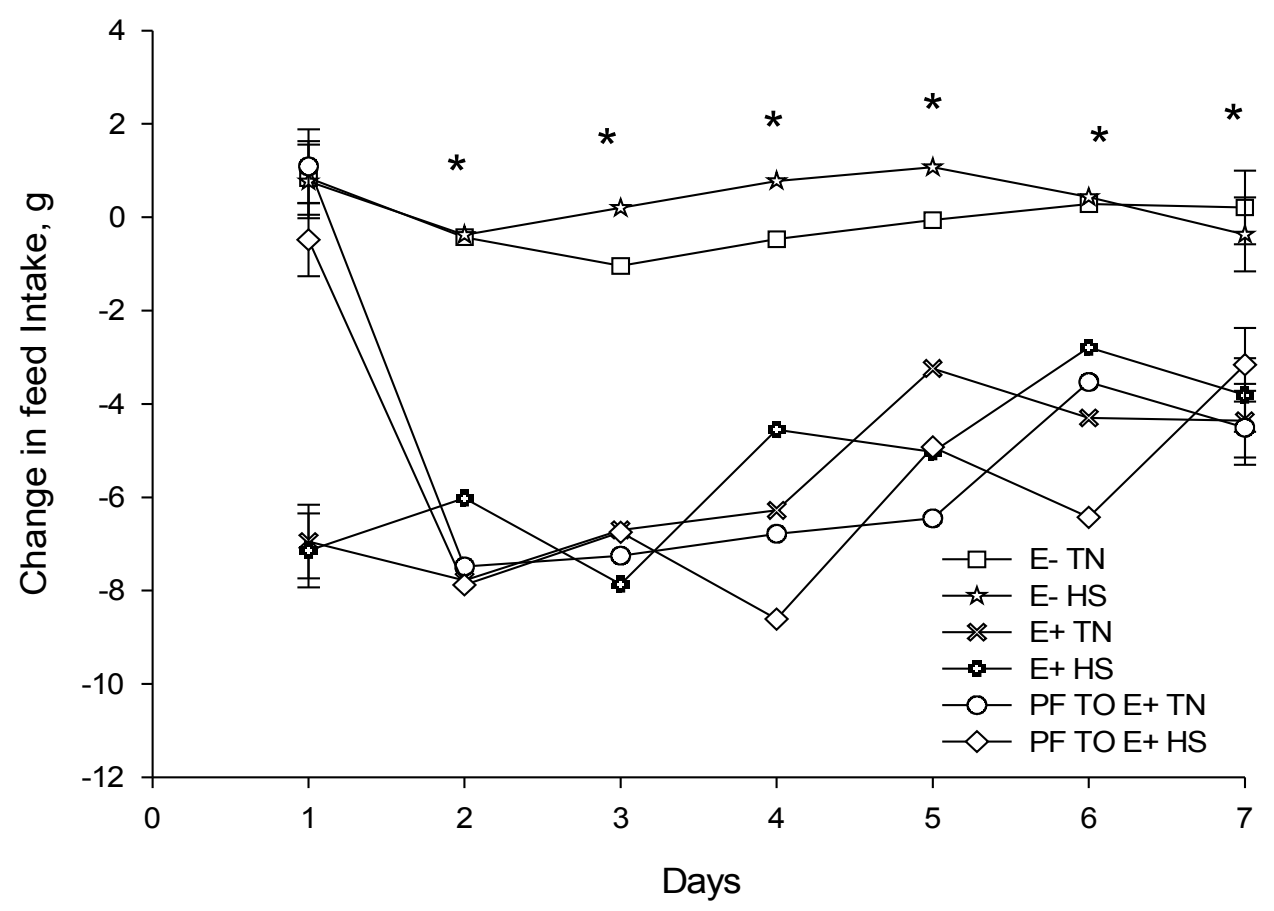

Figure 3.2. Change in average daily feed intake of E-, E+, and PF TO E+ during diet treatment period (Days 1 to 7 ). E- TN rats consumed $18 \mathrm{~g}$ on day -1 ..An asterisk (*) shows the statistical significance between E+ HS and E- TN groups. The vertical lines above and below the first and last points indicate \pm SE. 


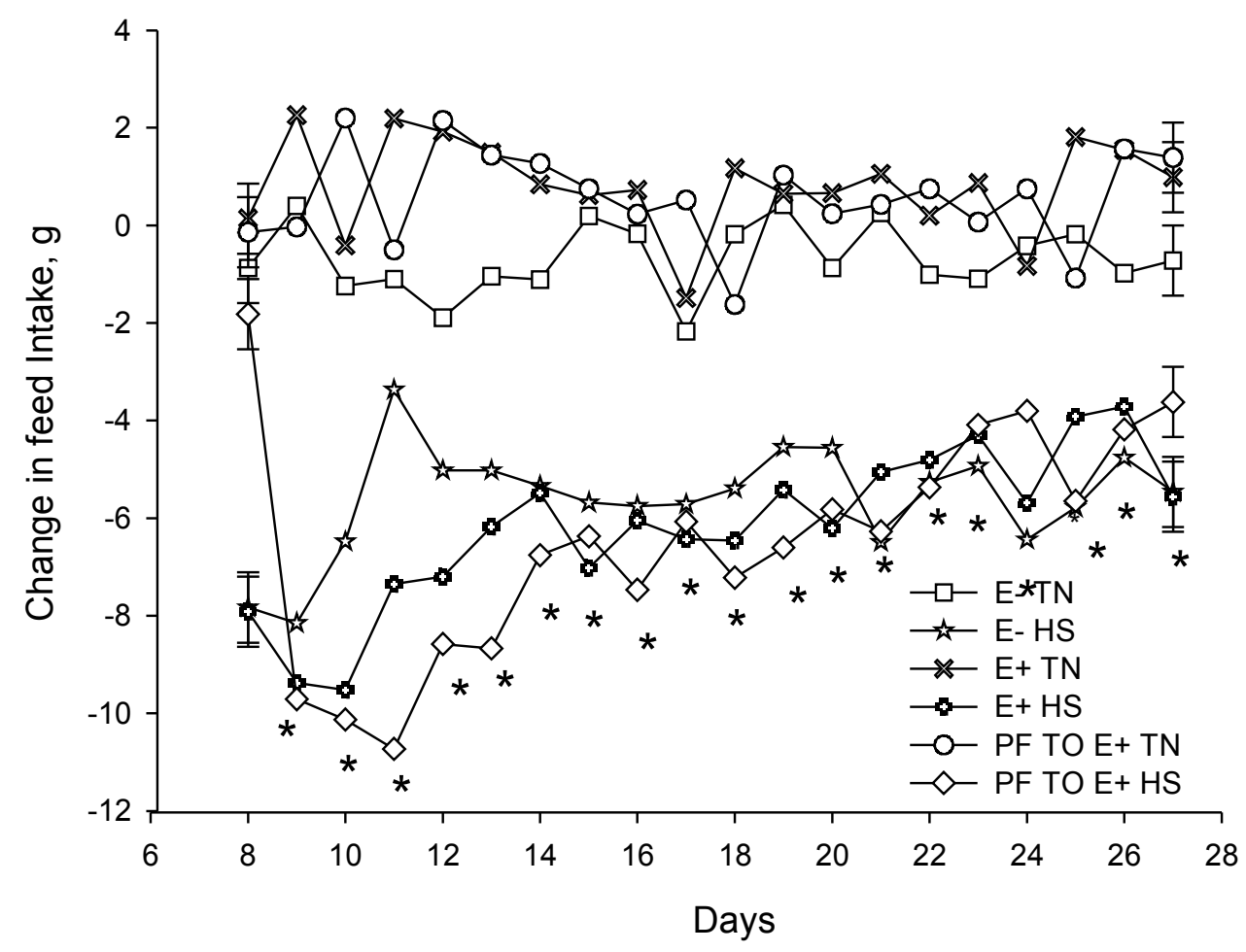

Figure 3.3. Change in average daily feed intake of E-, E+, and PF TO E+ during heat stress and diet treatment period (Days 8 to 28). E- TN rats consumed $18 \mathrm{~g}$ on day 7. An asterisk $(*)$ shows the statistical significance between E+ HS and E- TN groups. The vertical lines above and below the first and last points indicate \pm SE. 


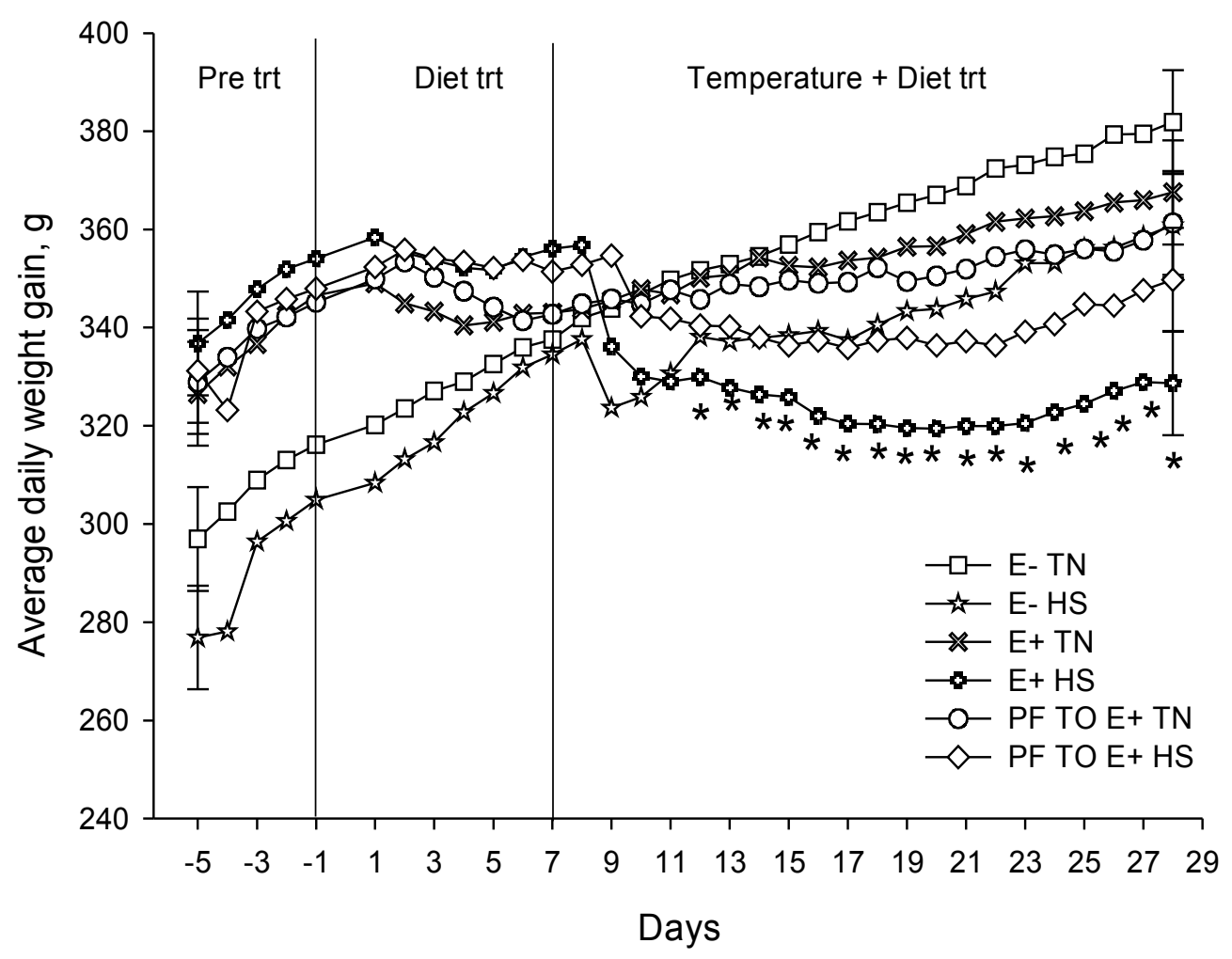

Figure 3.4. Average daily body weight gain of E-, E+, and PF TO E+ rats maintained under HS and TN conditions. An asterisk $(*)$ shows the statistical significance between E+HS and E-TN rats. The vertical lines above and below the first and last points indicate \pm SE. 


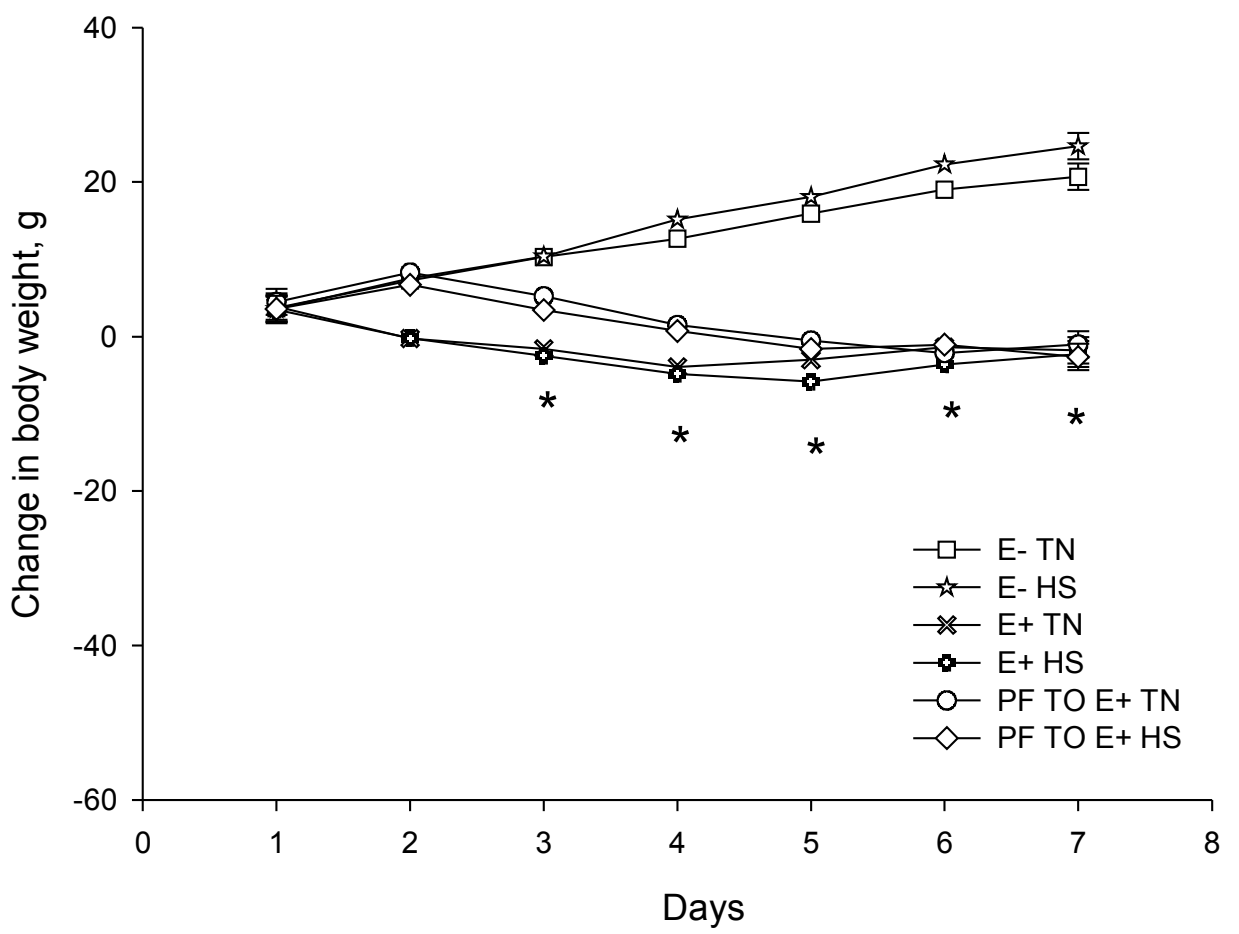

Figure 3.5. Change in average daily body weight intake of E-, E+ and PF TO E+ during diet treatment period (Days 1 to 7 ). Asterisk (*) shows the statistical significance between E+TN and E-TN rats. The vertical lines above and below the first and last points indicate $\pm \mathrm{SE}$. 


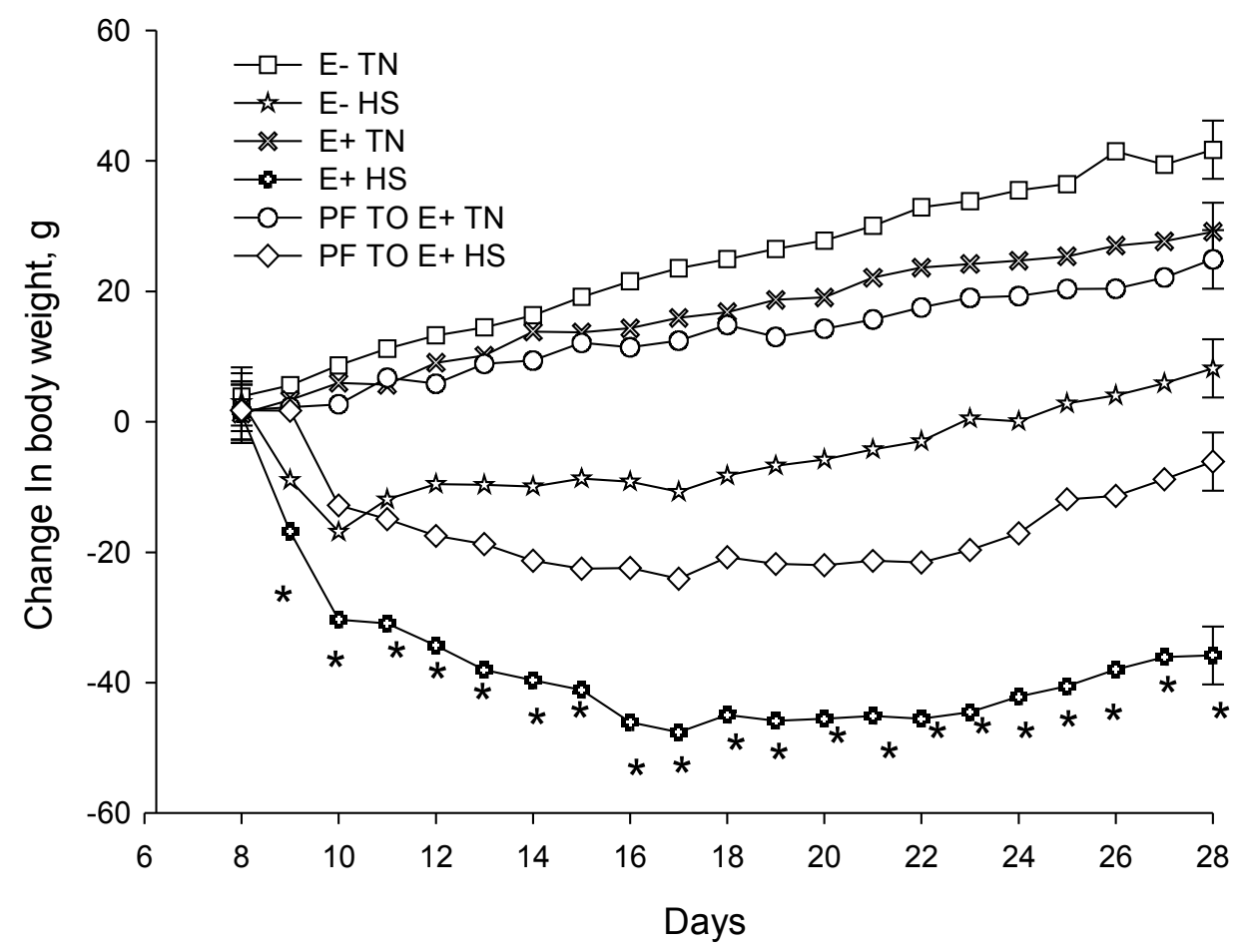

Figure 3.6. Change in average daily body weight of E-, E+, and PF TO E+ during HS and diet treatment period (Days 8 to 28). An asterisk (*) shows the statistical significance between E+HS and E-TN rats. The vertical lines above and below the first and last points indicate \pm SE. 


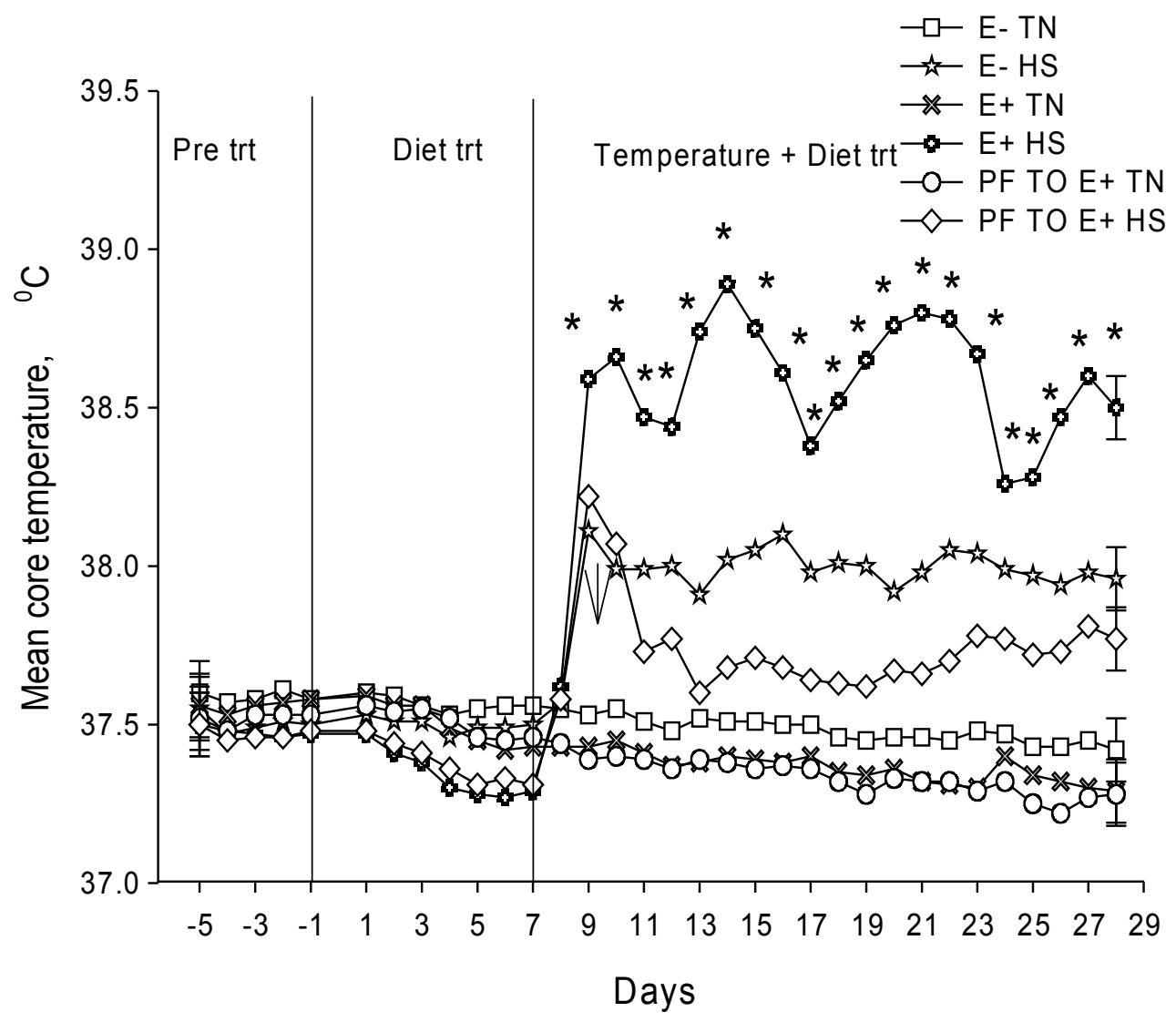

Figure 3.7. Average daily core body temperature of E-, E+, and PF TO E+ rats maintained under $\mathrm{HS}$ and TN conditions during entire study period. An asterisk (*) shows the statistical significance between E+HS and E-TN rats. The vertical lines above and below the first and last points indicate \pm 1 SE. 


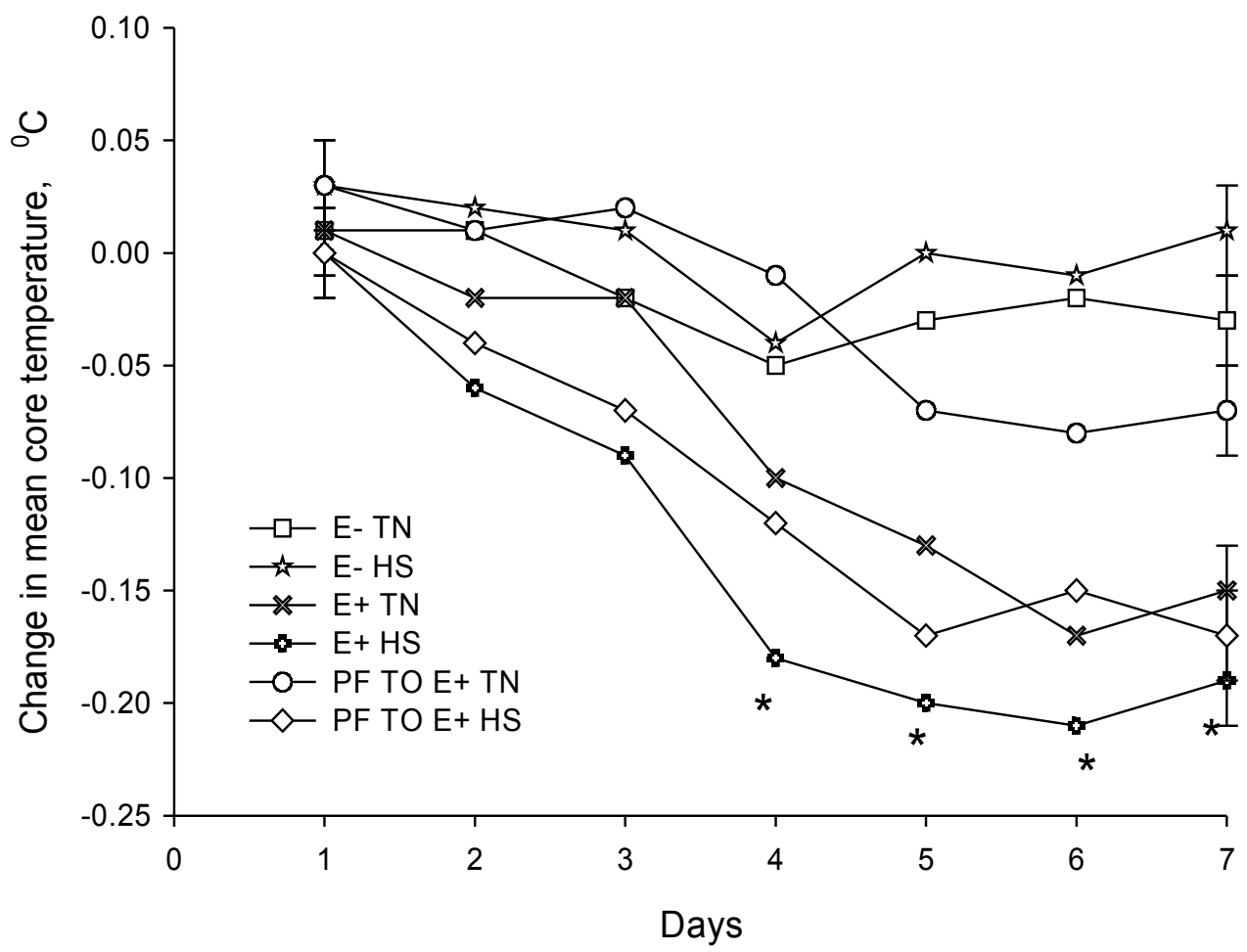

Figure 3.8. Change in average daily core body temperature of E-, E+, and PF TO E+ during diet treatment. An asterisk $(*)$ shows the statistical significance between E+TN and E-TN rats. The vertical lines above and below the first and last points indicate $\pm 1 \mathrm{SE}$. 


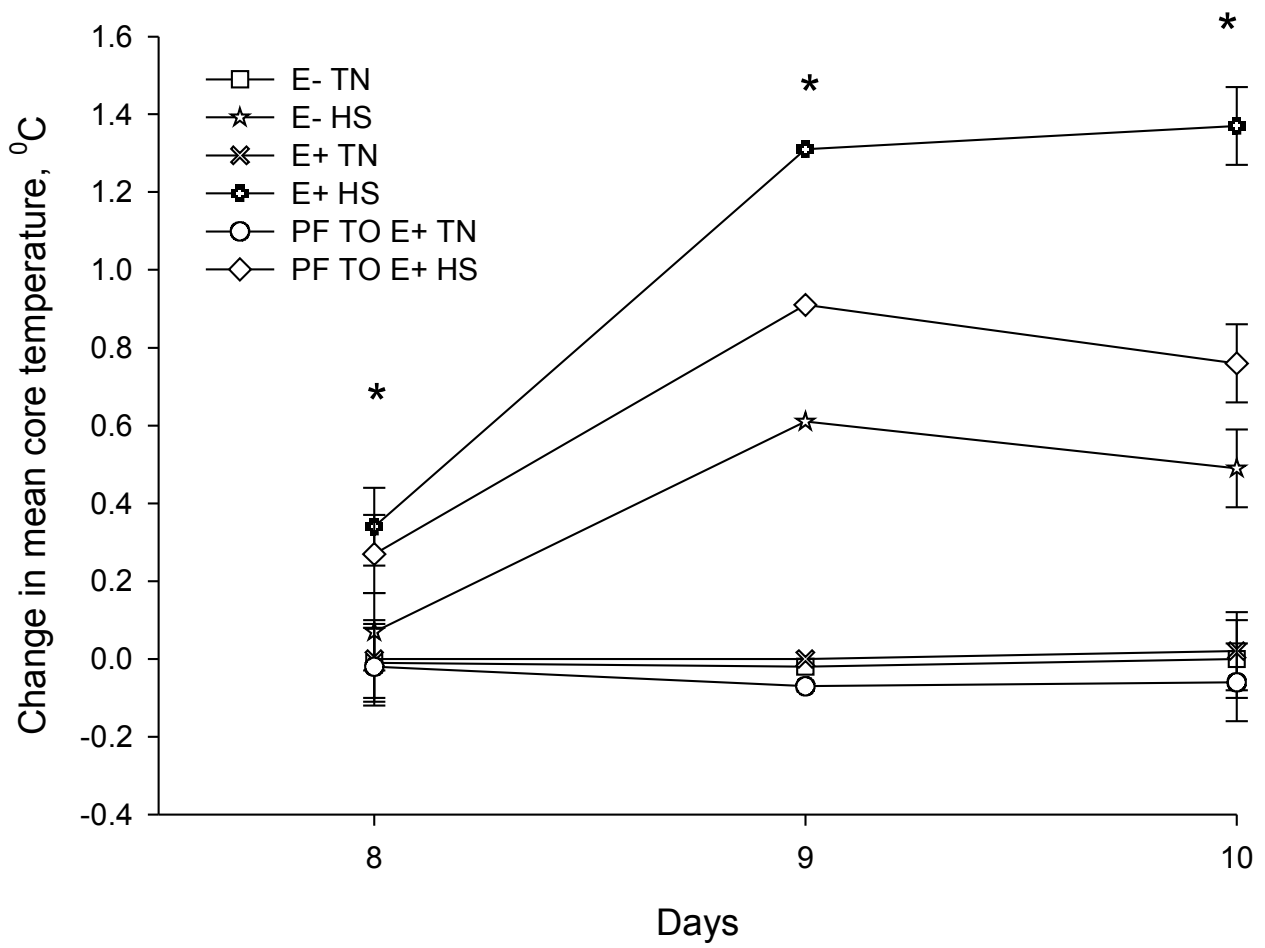

Figure 3.9. Change in core body temperature of E-, E+, and PF TO E+ during first three days of HS and diet treatment period (Days 8 to 10). An asterisk $(*)$ shows the statistical significance between E+HS and E-TN rats. The vertical lines above and below the first and last points indicate \pm 1 SE. 


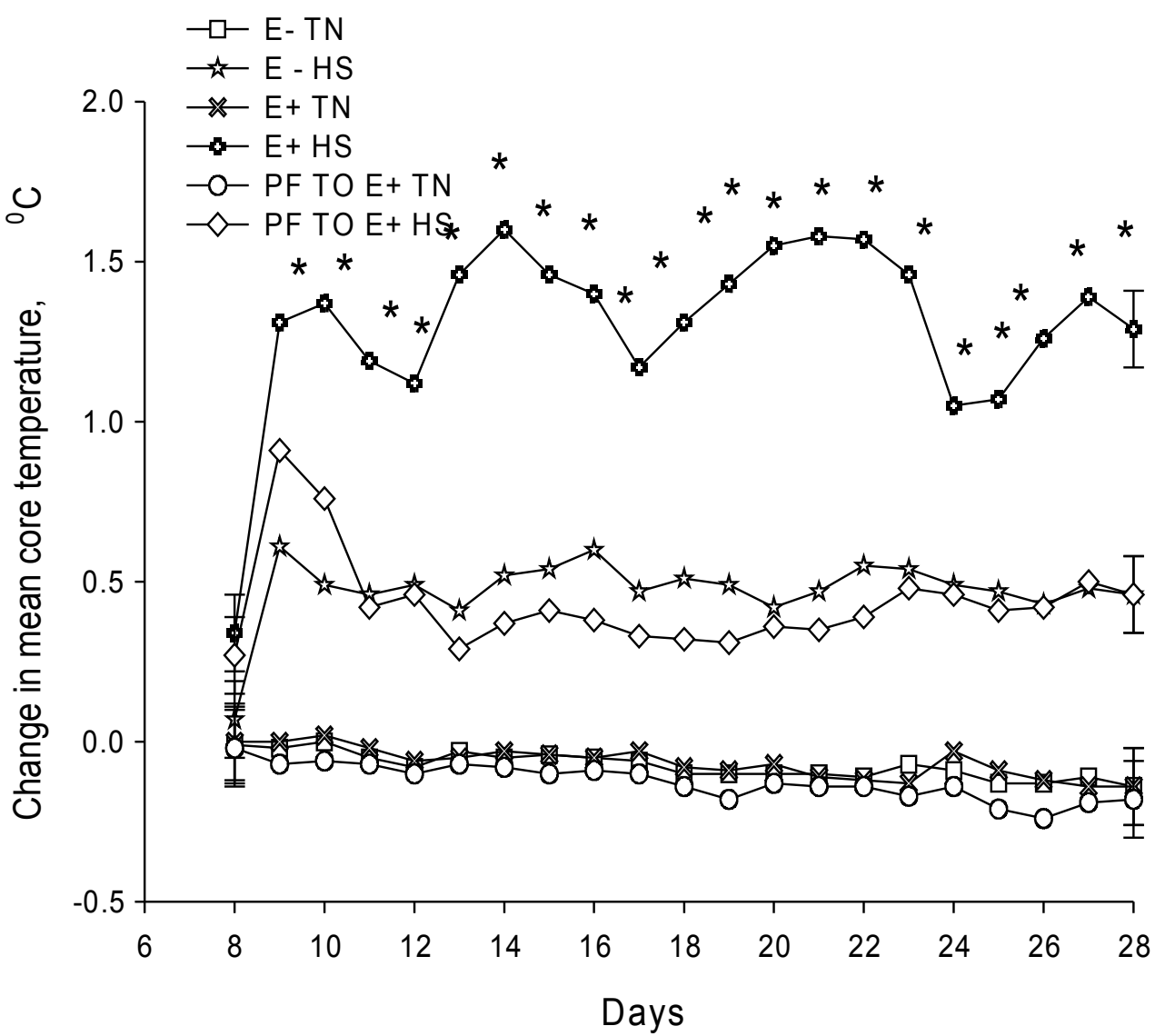

Figure 3.10. Change in core body temperature of E-, E+, and PF TO E+ of rats maintained under HS and TN conditions (Days 8 to 28). An asterisk (*) shows the statistical significance between E+HS and E-TN rats. The vertical lines above and below the first and last points indicate \pm 1 SE. 


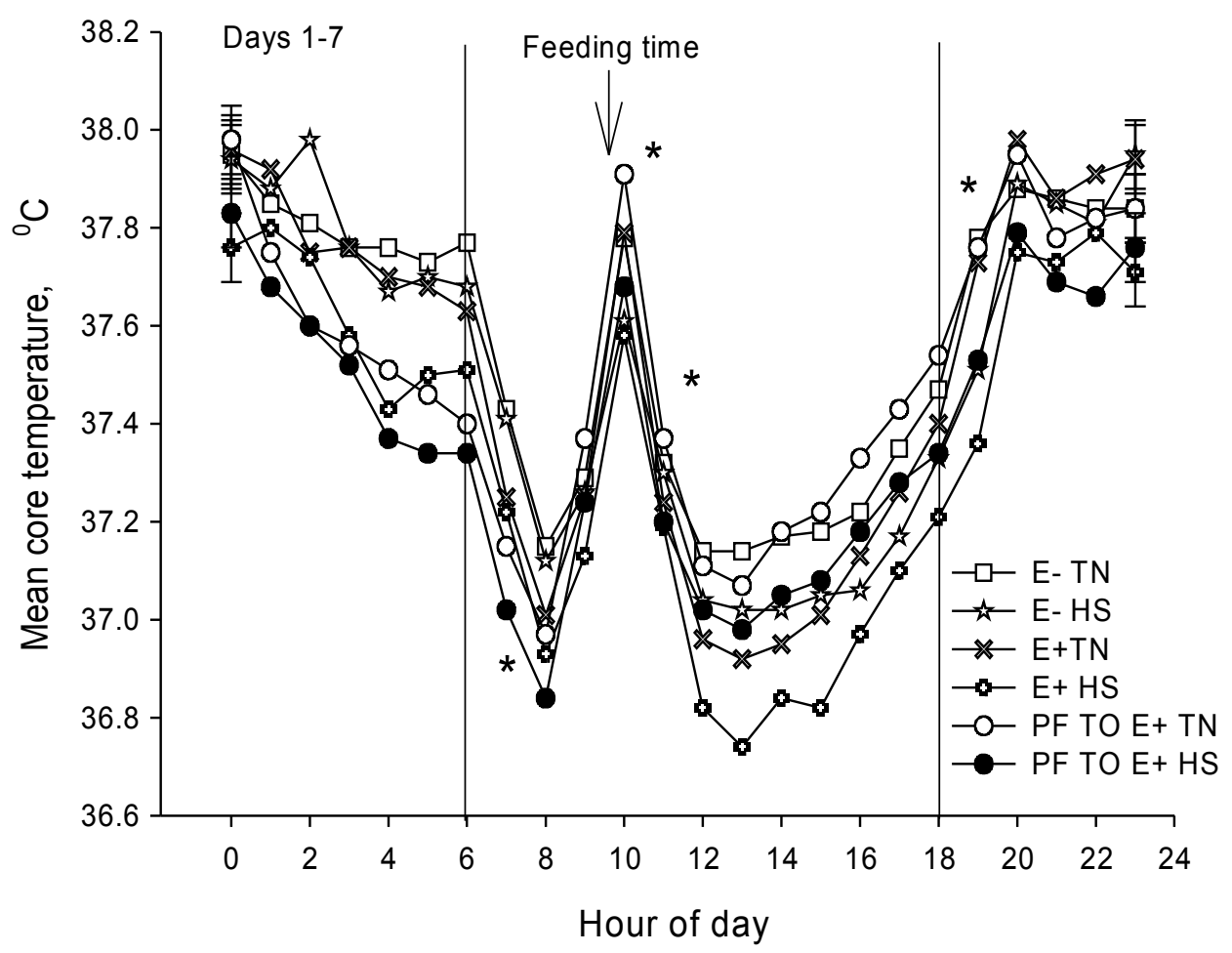

Figure 3.11. Circadian changes in core body temperature of E-, E+, and PF TO E+ rats during diet treatment period (Days 1 to 7). Vertical lines show periods when lights were turned on (0600) and when lights were turned off (1800). An asterisk $(*)$ shows the statistical significant difference from previous hour of day. The vertical lines above and below the first and last points indicate $\pm 1 \mathrm{SE}$. 


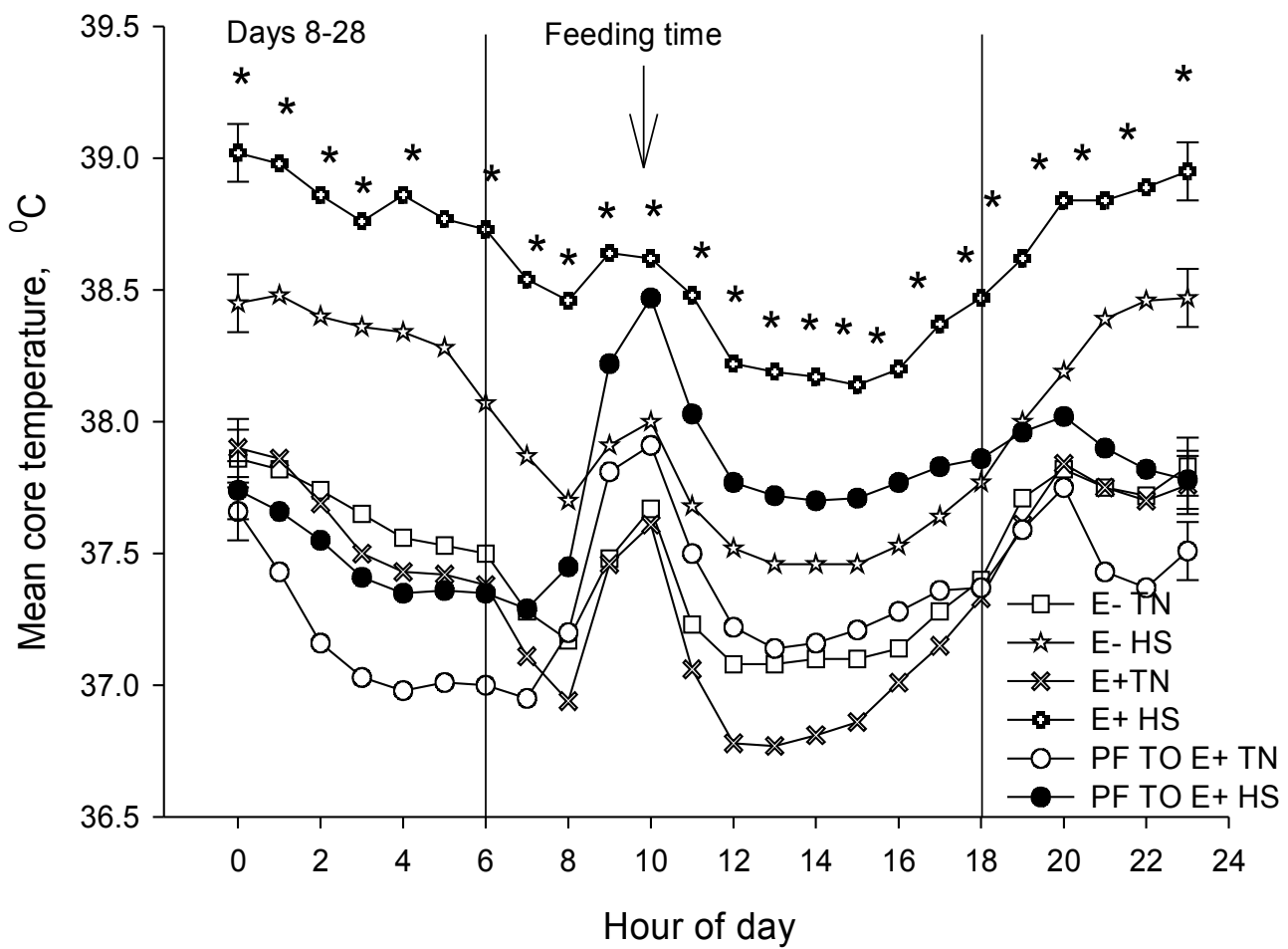

Figure 3.12. Circadian changes in core body temperature of E-, E+, and PF TO E+ rats maintained under HS and TN conditions (Days 8 to 28). Vertical lines show periods when lights were turned on (0600) and when lights were turned off $(1800)$. An asterisk $(*)$ shows the statistical significance between $\mathrm{E}+\mathrm{HS}$ and E-TN rats during 24 hour period. The vertical lines above and below the first and last points indicate \pm 1 SE. 


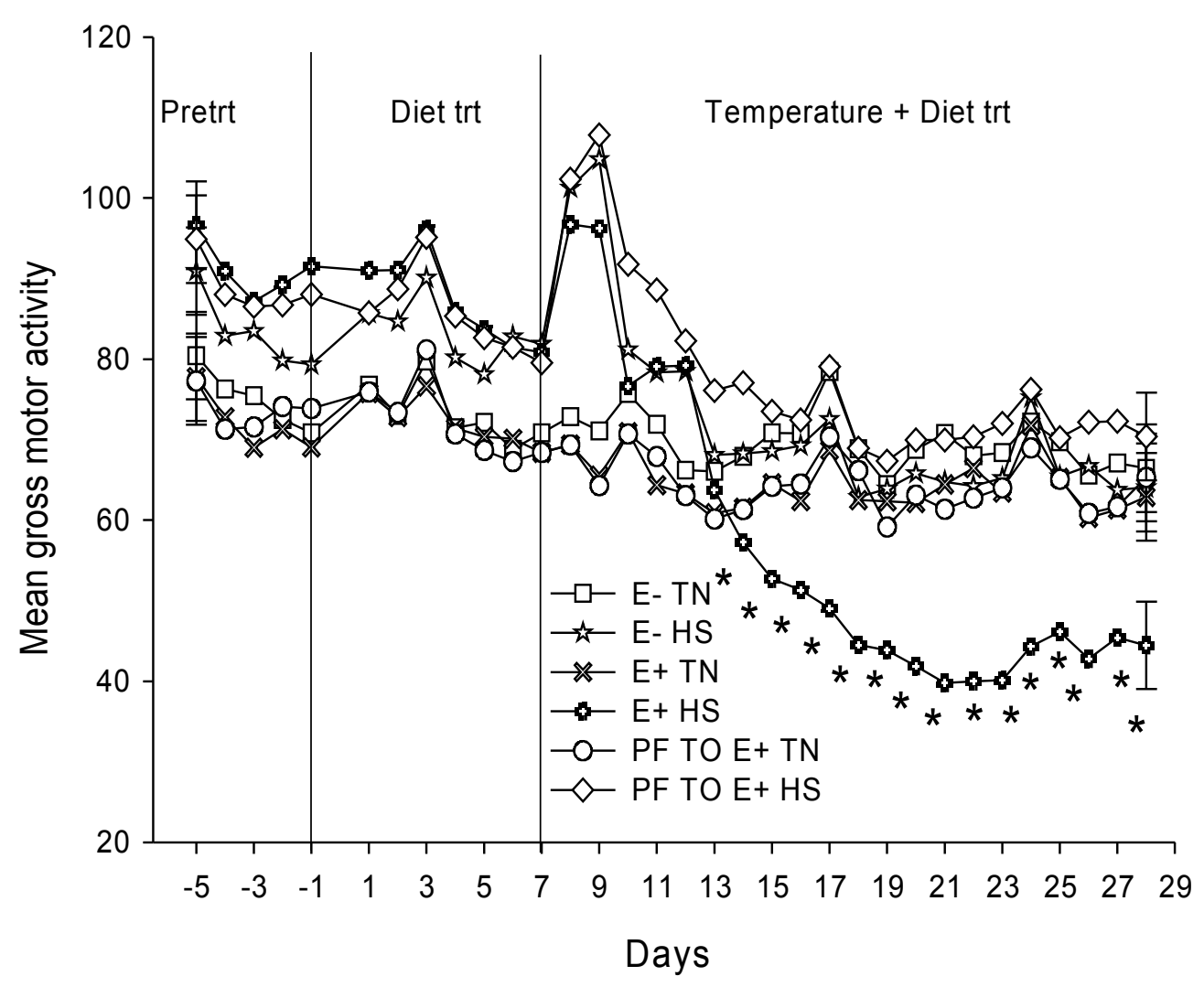

Figure 3.13. Average daily activity of E-, E+, and PF TO E+ rats maintained under HS and TN conditions. An asterisk $(*)$ shows the statistical significance between E+HS and E-TN rats. The vertical lines above and below the first and last points indicate $\pm 1 \mathrm{SE}$. 


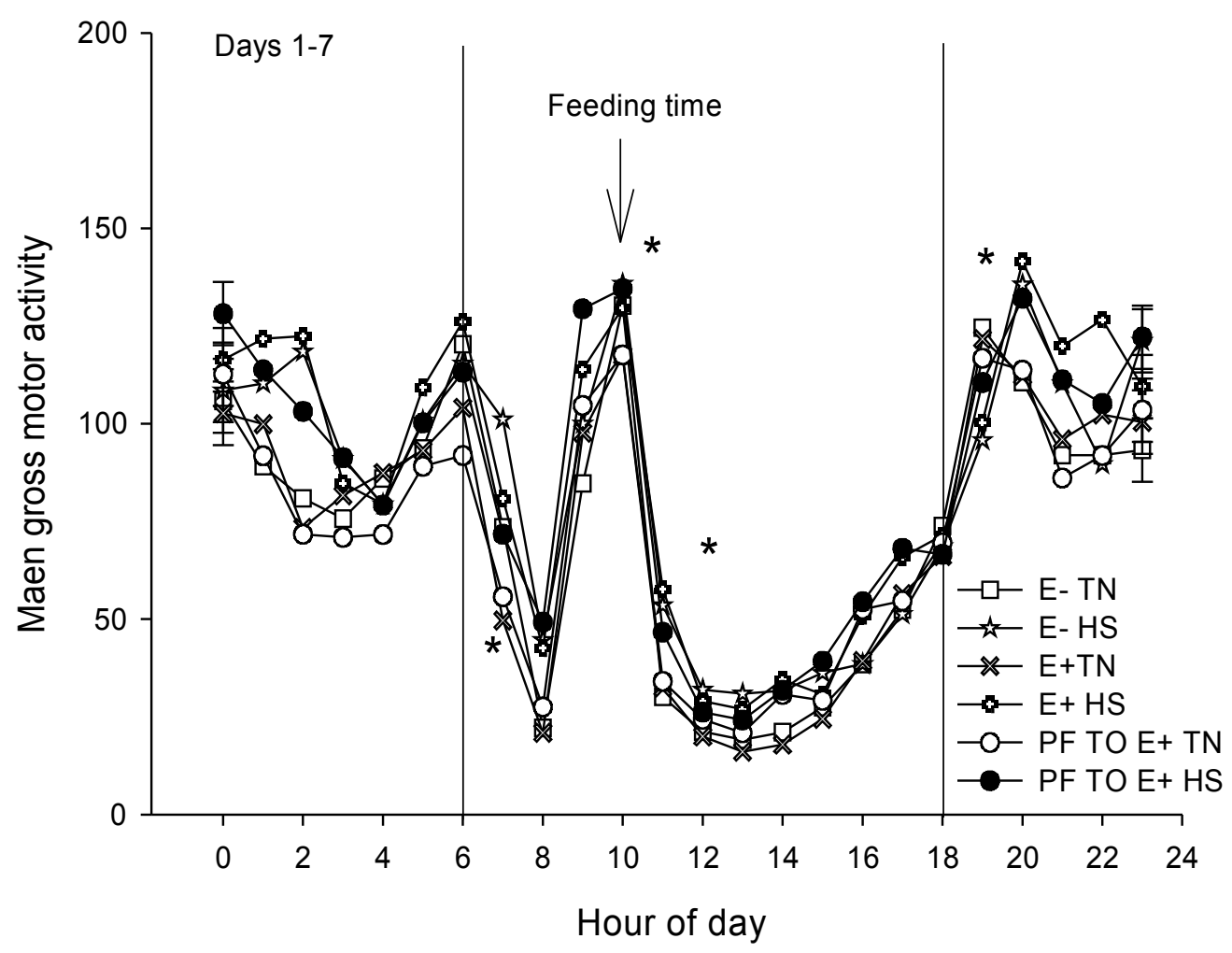

Figure 3.14. Circadian changes in average daily activity of E-, E+, and PF TO E+ rats during diet treatment period (Days 1 to 7). Vertical lines show periods when lights were turned on (0600) and when lights were turned off (1800). An asterisk $\left(^{*}\right)$ shows the statistical significant difference from previous hour of day. The vertical lines above and below the first and last points indicate $\pm 1 \mathrm{SE}$. 


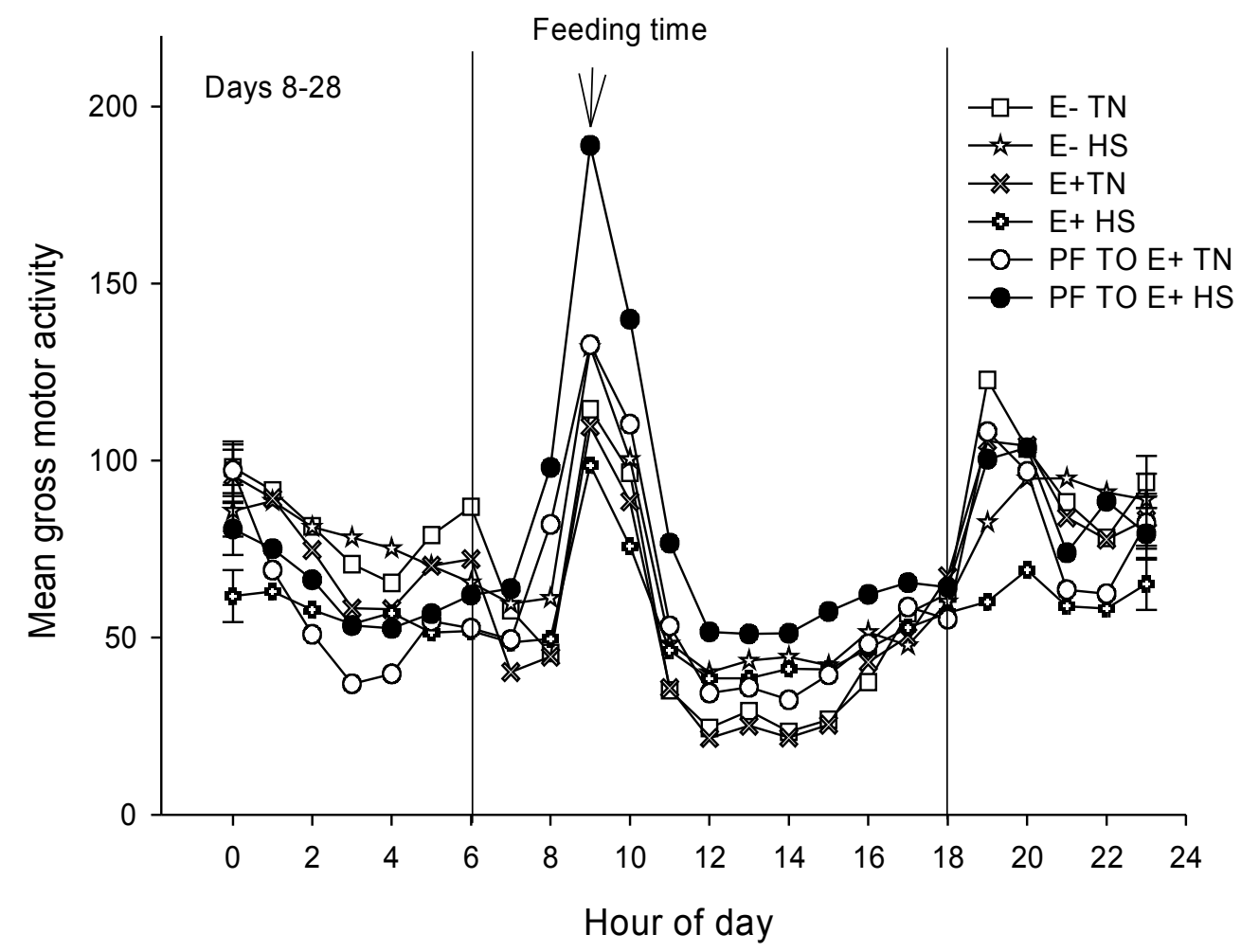

Figure 3.15. Circadian changes in average daily activity of E-, E+, and PF TO $\mathrm{E}+$ rats maintained under HS and TN conditions (Days 8 to 28). Vertical lines show periods when lights were turned on (0600) and when lights were turned off (1800). The vertical lines above and below the first and last points indicate $\pm 1 \mathrm{SE}$. 


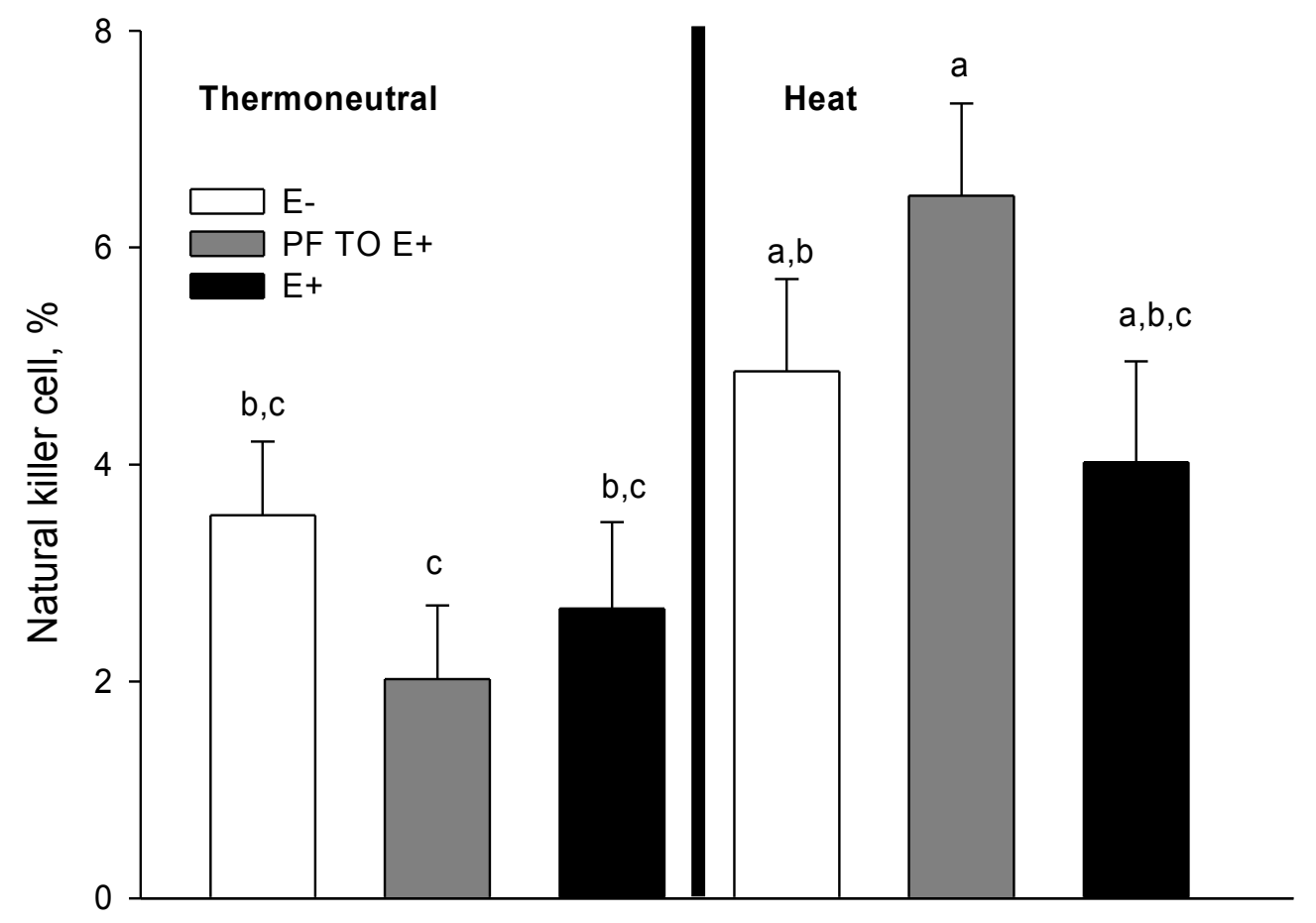

Figure 3.16. Horizontal bars represent E-, E+, and PF TO E+ percentages of natural killer Cells maintained under TN and HS conditions. The vertical lines above each bar indicates +1 SE. A heat stress effect $(P>0.002)$ was observed, but an endophyte $(\mathrm{P}>0.52)$ effect was not seen. 


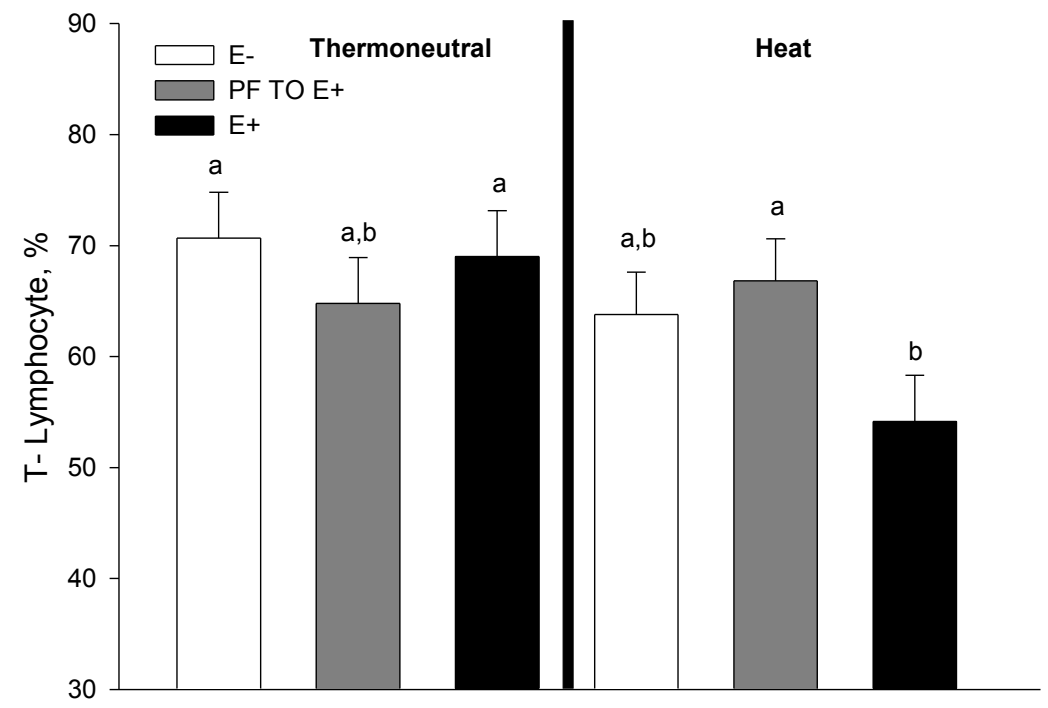

Figure 3.17. Horizontal bars represent E-, E+, and PF TO E+ percentages of T-lymphocytes maintained at TN and HS conditions. Different letters above each bar indicate significance across treatment groups. The vertical lines above each bar indicates +1 SE. A heat stress effect $(P>0.04)$ was observed, but an endophyte $(\mathrm{P}>0.33)$ effect was not seen. 


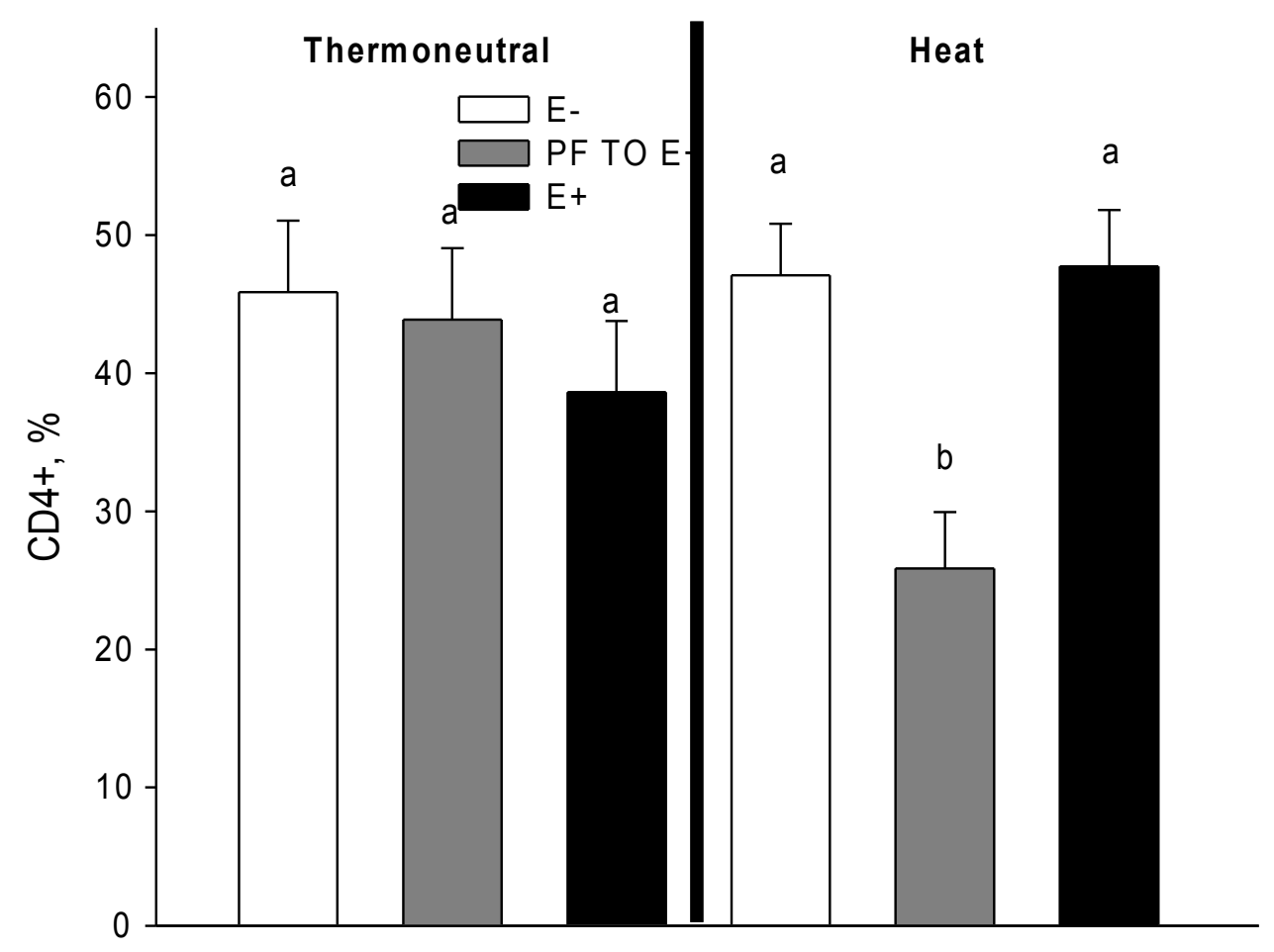

Figure 3.18. Horizontal bars represent E-, E+, and PF TO E+ percentages of CD4+ cells maintained under TN and HS conditions. Different letters above each bar indicate significance across treatment groups. The vertical lines above each bar indicates $+1 \mathrm{SE}$. An endophyte effect $(\mathrm{P}>0.03)$ was observed, but a heat effect $(\mathrm{P}>0.59)$ effect was not seen. 


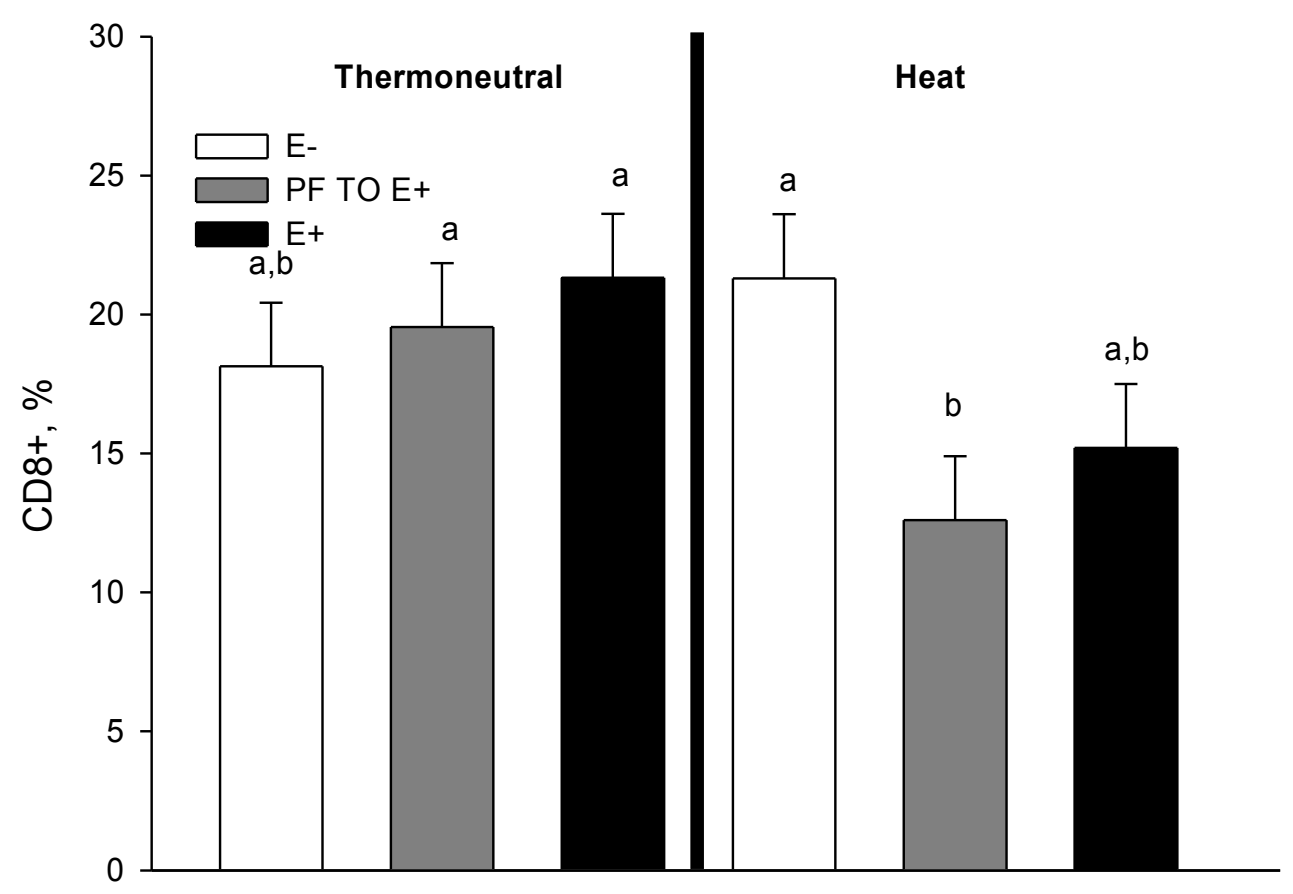

Figure 3.19. Horizontal bars represent E-, E+, and PF TO E+ percentages of $\mathrm{CD} 8+$ cells maintained under TN and HS conditions. Different letters above each bar indicate significance across treatment groups. The vertical lines above each bar indicates +1 SE. A heat stress effect $(P>0.09)$ and endophyte effect $(\mathrm{P}>0.29)$ was not observed. 


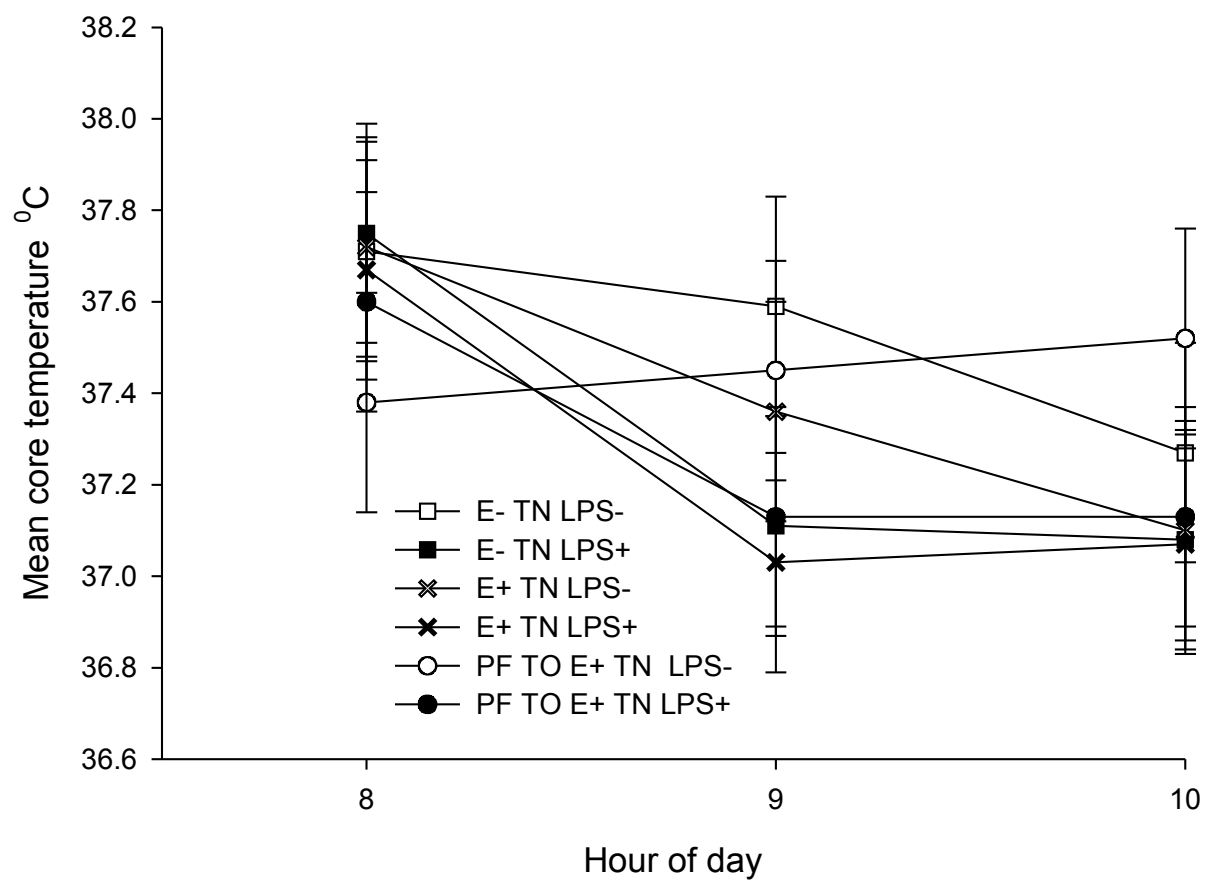

Figure 3.20. Average core body temperature of E-, E+, and PF TO E+ rats in response to LPS at thermoneutrality. The vertical lines above and below each point indicates $\pm 1 \mathrm{SE}$. 


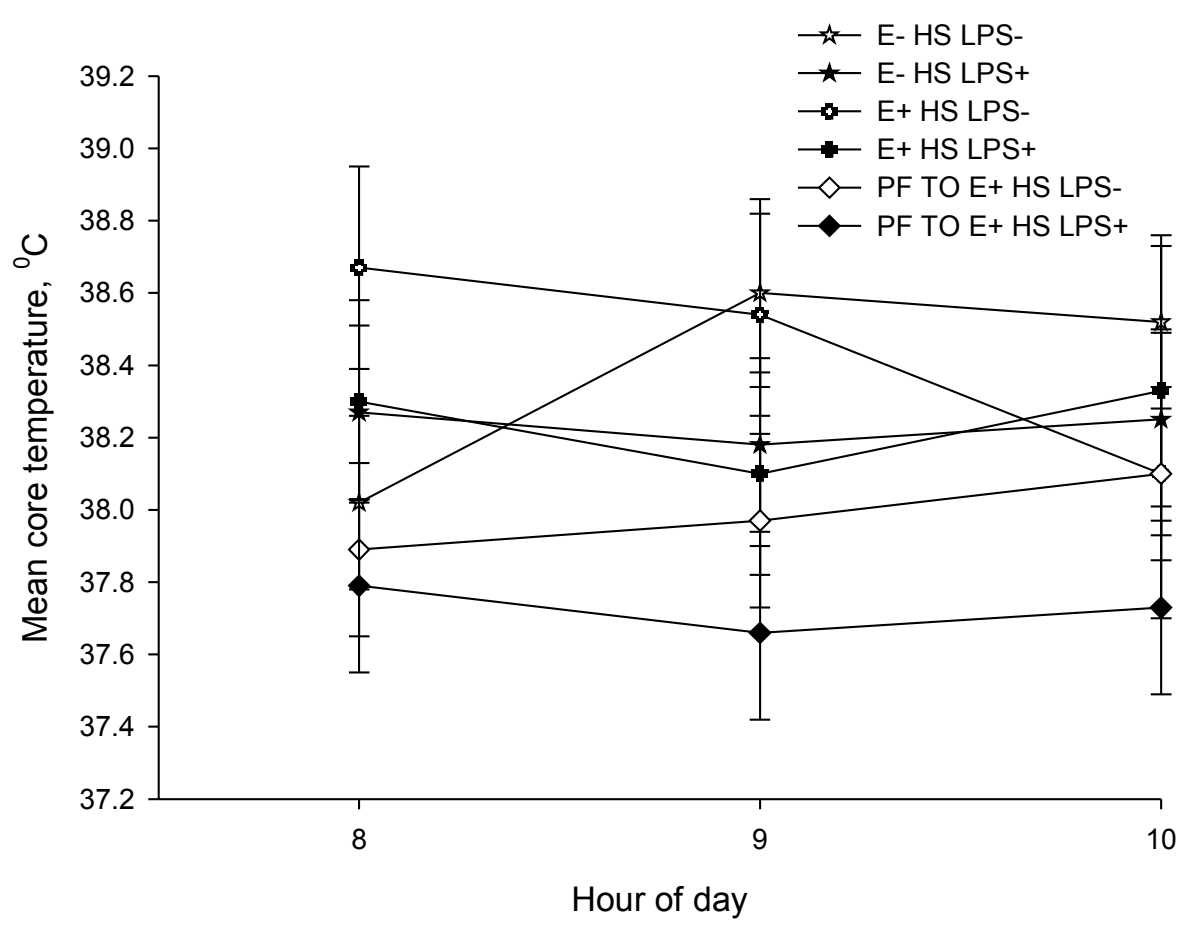

Figure 3.21. Average core body temperature of E-, E+, and PF TO E+ rats in response to LPS under heat stress conditions. The vertical lines above and below each point indicates $\pm 1 \mathrm{SE}$. 


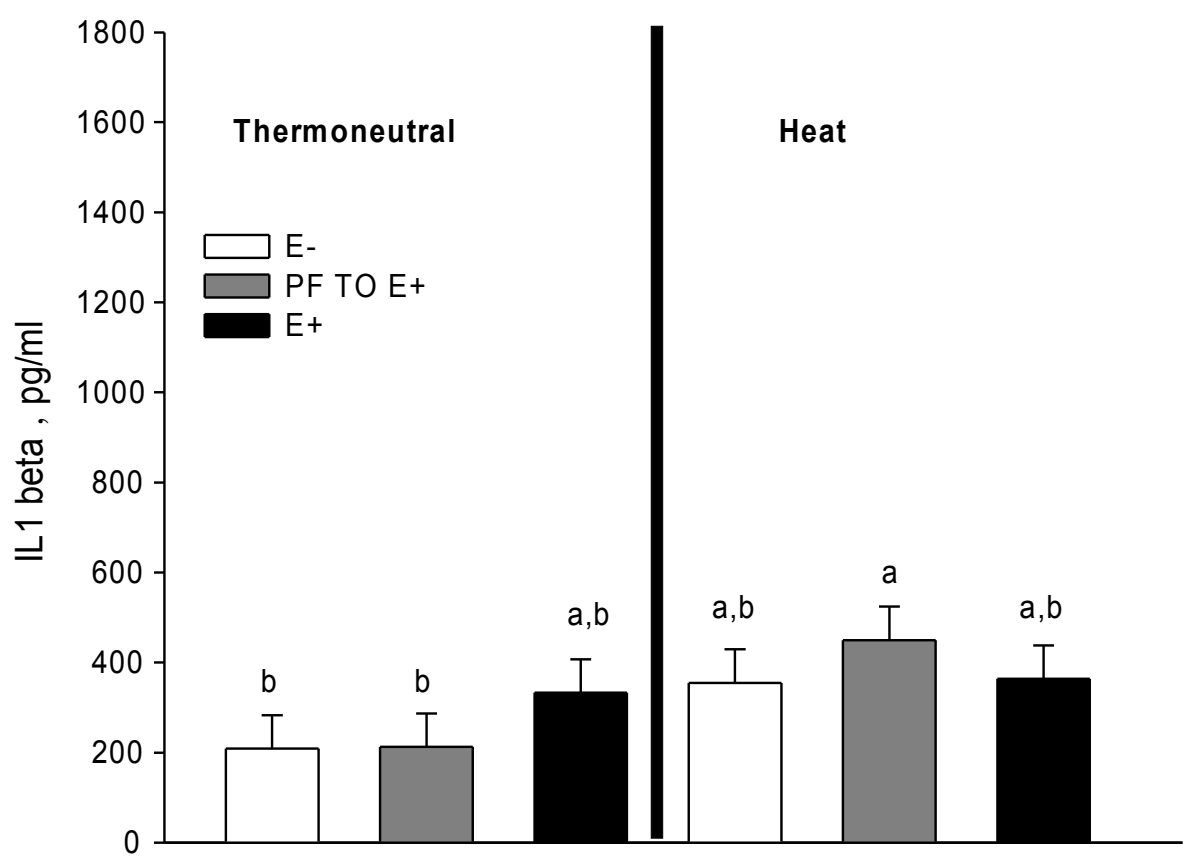

Figure 3.22. Horizontal bars represent levels of circulating IL1 $\beta$ in E-, E+, and PF TO E +2 hours after LPS challenge at TN and under HS conditions. Different letters above each bar indicate significance across treatment groups. The vertical lines above each bar indicates $+1 \mathrm{SE}$. A heat effect $(\mathrm{P}>0.03)$ was observed, but a endophyte effect $(\mathrm{P}>0.66)$ was not seen. 


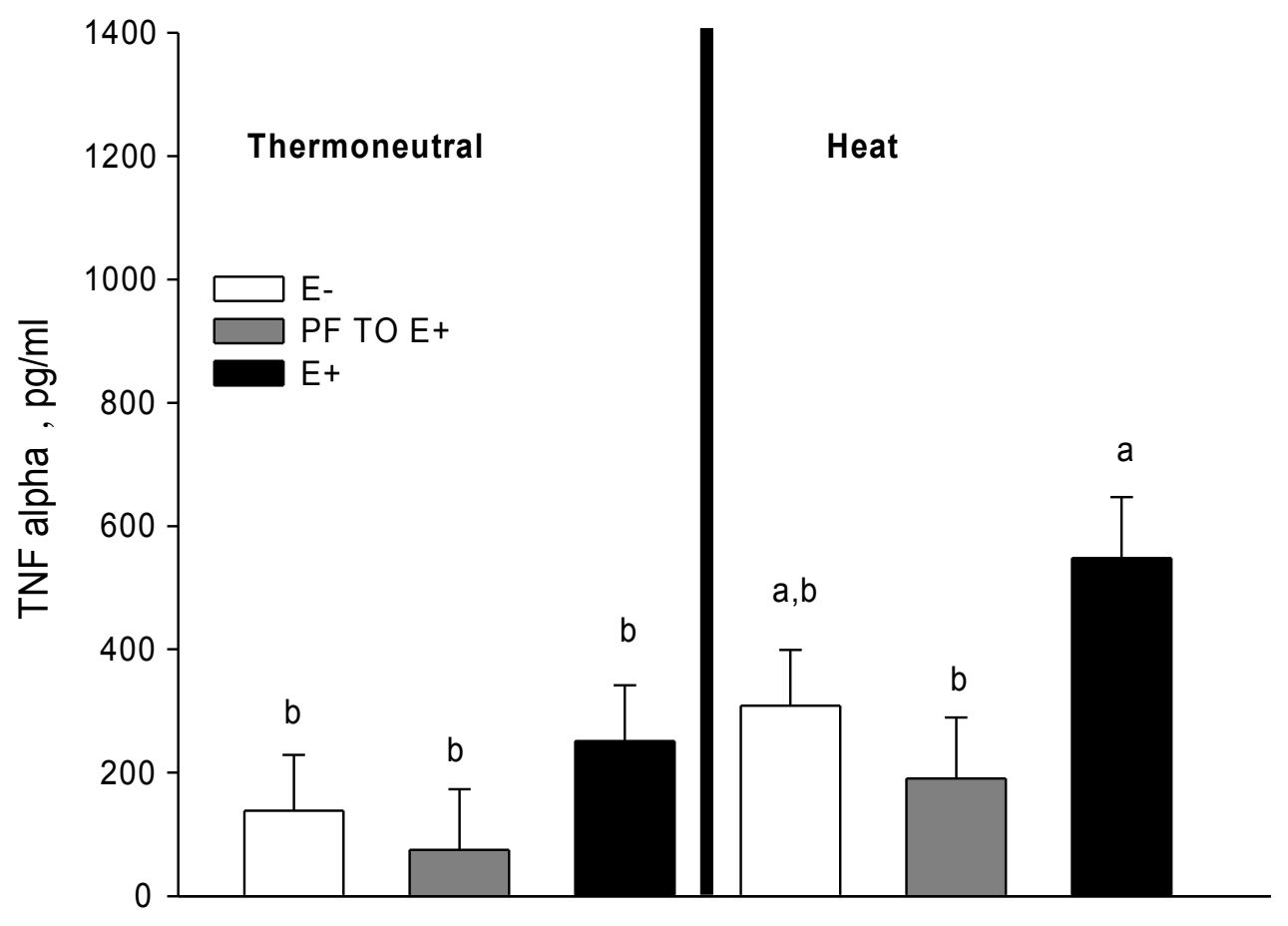

Figure 3.23. Horizontal bars represent levels of circulating TNF $\alpha$ in E-, E+ and PF TO E+ 2 hours after LPS challenge at TN and under HS conditions. Different letters above each bar indicate significance across treatment groups. The vertical lines above each bar indicates $+1 \mathrm{SE}$. A heat effect $(\mathrm{P}$ $>0.01)$ and endophyte effect $(\mathrm{p}>0.03)$ was observed. 


\section{Summary}

The current study was designed to examine a portion of the immune system response to fescue toxicosis, and the potentially compounding effects of heat stress and caloric restriction on this activity. The results differentiate the effects of these individual stressors, as well as their interactive actions on different physiological parameters and specific components of the immune system.

In the short-term study, rats decreased their feed intake and body weight gain, and increased their core body temperature in response to heat stress and a combination of heat stress and endophyte infected diet (Table. 1.2). These variables did not change until the end of the study.

In the long-term study, rats exposed to heat stress and either fed an endophytefree diet or pair-fed showed signs of adaptation. After three days in the heat, these rats increased their feed intake and body weight gain. Their core body temperature was also reduced at this time and stabilized slightly above the thermoneutral level. In contrast, rats receiving the endophyte-infected diet in the heat had a lower feed intake and body weight gain until the last day of study, and did not show an adaptive response comparable to other treatment groups in the heat (Table. 1.3). Likewise, their core body temperature was higher than other treatment groups in the heat and did not show signs of adaptation. This shows that the combination of these stressors hampers adaptation of core body temperature. If similar effects are experienced by farm animals, economic losses may occur during summer months. Our first study has shown that sensitizing rats to ergopeptine alkaloids, improves the response to a combination of heat stress and fescue toxicosis. The same approach might be used for farm animals before exposure to summer heat and endophyte-infected pastures to improve heat tolerance under these conditions. 
The immune responses to short-term heat stress included an initial increase in Tlymphocytes of heat-stressed rats on the endophyte-infected diet. However, there was a significant suppression of T-lymphocytes in the long-term study (Table. 1.3). This might indicate an impairment in cell-mediated immunity, making the animal susceptible to intracellular and extracellular pathogens. During short-term heat stress, the B-lymphocyte percentages of heat-stressed rats on the endophyte-infected diet had the lowest percentage among all the treatment groups. This could reduce their ability to produce antibodies, making them susceptible to extracellular pathogens. In the long-term study, B-lymphocyte percentage did not differ across treatment groups, suggesting that long-term exposure to heat stress on an endophyte-infected diet may not affect antibody production.

Cytokine response was maximal in heat-stressed rats on the endophyte-infected diet for both short- and long-term studies. Heat-stressed rats on the endophyte- free diet also experienced an increased cytokine release. This response shows that intake of ergopeptine alkaloids and heat stress does not attenuate the pro-inflammatory response. In contrast, these stressors may produce an increase in the levels of circulating pro-inflammatory cytokines in response to infection. However, an increased stimulation of the pro-inflammatory response may also be harmful to host tissues. This study only examined blood samples at one point following lipopolysaccharide injection, and could have missed some of the rise or fall in response. Thus, blood samples collected at different time points in future studies might provide a better understanding of the pro-inflammatory response which is a dynamic process.

Caloric restriction during short-term heat stress did not produce significant changes in lymphocyte percentages. However, long-term caloric restriction reduced 
CD4+ and CD8+ cells, possibly making the animal susceptible to both intracellular and extracellular pathogens. The lower levels of pro-inflammatory cytokines in pairfed rats was seen as a positive adaptive mechanism to reduce chronic inflammatory response. Caloric restriction also helped rats adapt better to heat stress and maintain a lower core body temperature compared to rats that received the ad libitum endophyte-free diet in the heat. We suggest that caloric restriction individually has the above mentioned positive effects when the animal is subjected to heat stress. However, the additive, synergistic, or antagonistic effects of caloric restriction in rats fed the endophyte-infected diet in the heat are very hard to speculate, since this is the first time that the effects of caloric restriction and fescue toxicosis on the immune system of rats has been studied.

Overall, the combination of heat stress and intake of the endophyte-infected diet had maximal impact on core body temperature and immune system in short- and longterm studies. Although B-lymphocyte percentage recovered, a significant suppression of T-lymphocyte was observed in the long-term study. This could compromise the animals cell- mediated adaptive immunity and make it susceptible to intracellular and extracellular pathogens. The changes observed in the pair-fed group suggested that caloric restriction also contributes to changes in T-lymphocyte lineages. Therefore, we conclude that it is the combination of ergopeptine alkaloids, heat stress, and caloric restriction that has the greatest impact on the immune system and thermoregulatory ability. 


\begin{tabular}{|c|c|c|c|c|c|}
\hline \multicolumn{6}{|c|}{ SHORT-TERM } \\
\hline & TC & FI & $\mathbf{B}$ & $\mathbf{T}$ & CD4+ \\
\hline E-HS & $\uparrow$ & $\downarrow$ & - & - & - \\
\hline $\mathbf{E}+\mathbf{H S}$ & 个个 & $\downarrow \downarrow$ & $\downarrow$ & $\uparrow$ & - \\
\hline PFTOE + HS & $\uparrow$ & $\downarrow$ & - & - & - \\
\hline
\end{tabular}




\begin{tabular}{|c|c|c|c|c|c|}
\hline \multicolumn{6}{|c|}{ LONG-TERM } \\
\hline & TC & FI & $\bar{B}$ & $\mathbf{T}$ & CD4+ \\
\hline E-HS & - & $\downarrow$ & - & - & - \\
\hline $\mathbf{E}+\mathbf{H S}$ & 个个 & $\downarrow \downarrow$ & - & $\downarrow \downarrow$ & - \\
\hline PFTOE + HS & - & $\downarrow$ & - & - & $\downarrow$ \\
\hline
\end{tabular}

Table 1.3. The arrows indicate significant increase or decrease when compared to the E-TN group $(\mathrm{P}<0.05)$. Double arrows indicate a stronger response compared to other treatment groups. 


\section{REFERENCES}

Abbas, K. B., A. H. Lichtman, and S. Pillai. 2007. Cellullar and Molecular Immunology. $6^{\text {th }}$ Ed. Saunders Elsevier. Philadelphia, PA.

Albright, J. L. and C. W. Alliston. 1972. Effects of varying the environment upon performance of dairy cattle. J. Anim. Sci. 32: 566-577.

Aldrich, C. G., J. A. Paterson, J. L. Tate, and M. S. Kerley. 1993. The effects of endophyte infected tall fescue consumption on diet utilization and thermal regulation in cattle. J. Anim. Sci. 71: 164-170.

Aiken, G. E., M. L. Looper, S. F. Tabler, D. K. Brauer, J. R. Strickland, and F. N. Schrick. 2006. Influence of stocking rate and steroidal implants on growth rate of steers grazing toxic tall fescue and subsequent physiological responses. J. Anim. Sci. 84: 1626-1632.

Aiken, G. E., B. H. Kirch, J. R. Strickland, L. P. Bush, M. L. Looper, and F. N. Sci Schrick. 2007. Hemodynamic responses of the caudal artery to toxic tall Sci fescue in beef heifers. J. Anim. Sci. 85: 2337-2345.

Al-Tamimi, H. J., P. A. Eichen, G. E. Rottinghaus, and D. E. Spiers. 2007. Nitric oxide supplementation alleviates hyperthermia induced by intake of ergopeptine alkaloids during chronic heat stress. J. Thermal Biol. 32: 179-187.

AnderRyst, K. M., G. Joubert, M. Steyn, H. Pieters, M. van der Westhuizen, M. van Staden, and C. Venter.1998. Correlation among total lymphocyte count, absolute CD4+ count, and CD4+ percentage in a group of HIV-1-infected south african patients. J. Acquried Immune Def. Human Retro. 19: 238-244. .

Baccari, F., H. D. Johnson, and G. L. Hahn. 1983. Environmental heat effects on growth, plasma T3 and postheat compensatory effects on Holstein calves. Proc. Soc. Exp. Biol. Med. 173: 312-318.

Baker, M.A. 1989. Effect of dehydration and rehydration on thermoregulatory sweating in goats. J. Physiol. Lond. 417: 421-435.

Bednar I, G. Forsberg, A. Linden, G. A. Qureshi, and K. Sodersten. 1991. Involvement of dopamine in inhibition of food intake by cholecystokinin octapeptide in male rats. J. Neuroendocrinol. 3: 491-496.

Beutler, B. A., W. Milsark, and A. Cerami. 1985. Passive immunization against cachectin/tumor necrosis factor protects mice from lethal effects of toxin. J. Immunol. 135: 3972.

Biron, C. A., P. van den Elsen, M. M. Tutt, P. Medveczky, V. Kumar, and C. Terhorst. 1987. Murine natural killer cells stimulated in vivo do not express the $\mathrm{T}$ cell receptor T3 \pm , or T3 genes. J. Immunol. 139: 1704-1710. 
Biron, C. A. and R. T. Gazzinelli. 1995. Effects of IL-12 on immune responses to microbial infections: a key mediator in regulating disease outcome. Curr. Opin. Immunol. 7: 485-496.

Biron, C. A., H. C. Su, and J. S. Orange. 1996. Function and regulation of natural killer (NK) cells during viral infections: characterization of responses invivo.Methods: Companion Methods Enzymol. 9: 379-393.

Biron, C. A. 1997. Activation and function of natural killer cell responses during viral infections. Curr. Opin. Immunol. 9: 24-34.

Bison, S., L. Caboni, R. Arban, S. Bate, P. Gerrard, and M. Razzoli. 2009.

Differential behavioural, physiological and hormonal sensitivity to LPS challenge in rats. Intern. J. Interferon, Cytokine, Mediator Research1: 1-13.

Blackwell, T. S. and J. W. Christman. 1997. The role of nuclear factor-kappa B in cytokine gene regulation. Am. J. Respir. Cell. Mol. Biol. 17: 3-9.

Blatteis, C. M., and Sehic, E. 1998. Cytokines and fever. Ann. NY Acad. Sci. NY.

Blatteis, C. M. 2000. The afferent signalling of fever. J. Physiol. 526: 653- 661.

Blazquez, N. B., S. E. Long, T. M. Mayhew, G. C. Perry, N. J. Prescott,,and C. M. Wathes. 1994. Rate of discharge and morphology of sweat glands in the perineal, lumbodorsal and scrotal skin of cattle. Res. Vet. Sci. 57: 277-284.

Bligh, J. and K. G. Johnson. 1973. Glossary of terms for thermal physiology. J. Appl. Physiol. 35: 941-961.

Bottomly, K. 1988. A functional dichotomy in CD4+ T lymphocytes. Immunol. Today 9: 268-277.

Bouchama A., R. S. Parhar, A. el-Yazigi, K. Sheth, S. al-Sedairy. 1991. Endotoxemia and release of tumor necrosis factor and interleukin 1 alpha in acute heatstroke. J. Appl. Physiol. 70: 2640-2644.

Burcham, J., M. Marmor, and N. Dubin. 1991. CD4\% is the best predictor of development of AIDS in a cohort of HIV-infected homosexual men. AIDS 5: 365-372.

Buono, M.J. and N.T. Sjoholm. 1988. Effect of physical training on peripheral sweat production. J. Appl. Physiol. 65: 811-814.

Chai, Z, S. Gatti, V. Poli , and T. Bartfai. 1996. Interleukin (IL)-6 gene expression in the central nervous system is necessary for fever response to lipopolysaccharide or IL-1b: a study on IL-6 deficient mice. J. Exp. Med. 183: 311-316. 
Christine, A., K. B. Biron, G. C. Nguyen, G. C. Pien, L. P. Cousens, and T. P.Salazar-Mather. 1999. Natural killer cells in anti viral defense: Function and regulation by innate cytokines. Ann.Rev. Immunol. 17: 189-220.

Chu, L., E. K. Ribeiro, P. S.Slutsky and S. Arthur. 1997. Heat stress increases survival rates in lipopolysaccharide-stimulated rats. J. Crit. Care Medicine25: 1727-1732.

Cross, D. L., L. M. Redmond, and J. R. Strickland. 1995. Equine fescue toxicosis: Signs and solutions. J. Anim. Sci. 73: 899- 908.

Curtis, S. E. 1983. Perception of thermal comfort by farm animals. In: S. H. Baxter, M. R. Bnxter and J.A.D. MacCormack Ed. Farm Animal Welfare and Housing. Marlinus Nijhoff, Boston, MA.

De Rijk, R. H., M. Van Kampen, N. Van Rooijen, and F.Berkenbosch. 1994. Hypothermia to endotoxin involves reduced thermogenesis, macrophagedependent mechanisms and prostaglandins. Am. J. Physiol. 266: R1 - R8.

De Rijk, R.H. and F. Berkenbosch. 1996. Hypothermia to endotoxin involves the cytokine tumor necrosis factor and the neuropeptide vasopressin in rats. Am. J. Physiol. Regul. Integr. Comp. Physiol. 270: 693-703.

Dew, R. K., G. A. Boissonneault, N. Gay, J. A. Boling, R. J. Cross, and D. A. Cohen. 1990. The effect of the endophyte and associated toxin(s) of tall fescue on serum titer response to immunization and spleen cell flow cytometry analysis and response to mitogens. Vet. Immunol. Immunopathol. 26: 285-295.

De Vries, J., J. H. Strubbe, W. C. Wildering, J. A. Gorter, and A. J. A. Prins. 1993. Patterns of body temperature during feeding in rats under varying ambient temperatures. Physiol. Behav. 53: 229-235.

Dinarello, C.A., H.A. Bernheim, and G.W. Duff. 1984. Mechanisms of fever induced by recombinant human interferon. J. Clin. Invest. 74: 906-913.

Dinarello, C.A., J.G. Cannon, and S.M. Wolff.1986. Tumor necrosis factor (cachectin) is an endogenous pyrogen and induces production of interleukin-1. J .Exp. Med. 163: 1433-1450.

Dinarello, C. A. 1991. Inflammatory cytokines interleukin-1 and tumor necrosis factor as effector molecules in autoimmune diseases. Curr. Opin. Immunol. 3: 941- 948 .

Dracott, B.N. and C.E. Smith. 1979. Hydrocortisone and the antibody response in mice. I. Correlations between serum cortisol levels and cell numbers in thymus, spleen, marrow and lymph nodes. J. Immunol. 38: 429-435.

Duffy, P. H., R. J. Feuers, J. A. Leakey, K. Nakamura, A. Turturro, and R. W. Hart. 1989. Effect of chronic caloric restriction on physiological variables related to energy metabolism in the male fischer 344 rat. Mech. Ag. Dev. 48: 117-133. 
Ellis, S., A. Mouihate, and Q. J. Pittman. 2005. Early life immune challenge alters innate immune responses to lipopolysaccharide: implications for host defense as adults. The FASEB J. 10: 3569-3586.

El-Nouty, F. D., A. A. Al-Haidary, and M. S. Salah. 1990. Seasonal effects on body temperature, thyroid function, blood glucose and milk production in lactating and dry Holstein cows in semi-arid environment. Arab Gulf J. Sci. Res. 8: 89-103.

Esquifinoa, A.I., F. Chacona, P. Canoa, A. Marcosb, R. A. Cutrerac, D. P. Cardinali. 2004. Twenty-four-hour rhythms of mitogenic responses, lymphocyte subset populations and amino acid content in submaxillary lymph nodes of growing male rats subjected to calorie restriction. J. Neuro. Immunol. 156: 66-73.

Feldberg, W. and P. N. Saxena. 1975. Prostaglandins, endotoxin and lipid A on body temperature in rats. J. Physiol. Lond. 249: 601-615.

Filipov, N. M., F. N. Thompson, N. S. Hill, D. L. Dawe, J. A. Stuedemann, J. C. Price, and C.K. Smith. 1998. Vaccination against ergot alkaloids and the effect of endophyte-infected fescue seed-based diets on rabbits. J. Anim. Sci. 76: 2456 2463.

Filipov, N. M., F. N. Thompson, J. A. Stuedemann, T. H. Elsasser, S. Kahl, R. R. Sharma, C.R. Young, L. H. Stanker, and C. K. Smith. 1999a. Increased responsiveness to intravenous lipopolysaccharide challenge in steers grazing endophyte-infected tall fescue compared with steers grazing endophyte-free tall fescue. J. Endocrin. 163:213-220.

Filipov, N. M., F. N. Thompson, R. P. Sharma, and R. R. Dugyala. 1999b. Increased proinflammatory cytokines production by ergotamine in male BALB/c mice. J.Toxicol. Environ. Health. 58: 145-155.

Filipov, N. M., F. N. Thompson, J. A. Stuedemann, T. H. Elsasser, S. Kahl, L. H. Stanker, C.R. Young, D. L. Dawe, and C. K. Smith. 2000. Anti-inflammatory effects of ergotamine in steers. Proc. Soc. Exp. Biol. Med. 225: 136-142.

Flajnik, M.F. and L. Pasquier. 2004. Evolution of innate and adaptive immunity: Can we draw a line? Trends Immunol. 25: 640-644.

Freeman, M. 2000. Feedback control of intercellular signalling in development. Nature 408: 313-319.

Gabay, C. and I. Kushner. 1999. Acute-phase proteins and other systemic responses to inflammation. N. Engl. J. Med. 340: 448-454.

Gadberry, M. S., T. M. Denard, D. E. Spiers, and E. L. Piper. 2003. Effects of feeding ergovaline on lamb performance in a heat stress environment. J. Anim. Sci. 81: $1538-1545$.

Gidlund, M.O., H. Wigzell, A. Senik, and I. Gresser. 1978. Enhanced NK cell 
activity in mice injected with interferon and interferon inducers. Nature 273: 759-761.

Gioannini, T.L. and J.P. Weiss. 2007. Regulation of interactions of gramnegative bacterial endotoxins with mammalian cells. Immunol. Res. 39: 249260 .

Gonzalez, B. and R. Manso. 2004. Induction, modification and accumulation of HSP70 in the rat liver after acute exercise: early and late responses. J. Physiol. 556: 369-385.

Gordon, C. J. 1993. Thermoregulatory effector responses. In: Temperature Regulationin Laboratory Rodents. Cambridge Univ. Press, New York, NY.

Goujon,_E., S. Laye, P. Parnet, and R. Dantzer. 1997. Regulation of cytokine gene expression in the central nervous system by glucocorticoids: mechanisms and functional consequences. Psychoneuro. 22:75-88.

Harrington, L.E., R.D. Hatton, P.R. Mangan, H. Turner, T.L. Murphy, K.M. Murphy, and C.T. Weaver. 2005. Interleukin 17-producing CD4+ effector T cells develop via a lineage distinct from the $\mathrm{T}$ helper type 1 and 2 lineages. Nat. Immunol. 6:1123-1132.

Hawiger, J. 2001. Innate immunity and inflammation: a transcriptional paradigm. Immunol. Res. 23: 99-109.

Hemken, R.W., J. A. Boling, L. S. Bull, R. H. Hatton, R. C. Buckner, and L. P. Bush. 1981. Interaction of environmental temperature and anti-quality factors on the severity of summer fescue toxicosis. J. Anim. Sci. 52: 710-714.

Hill, N. S., F. N. Thomson, J. A. Stuedemann, G. W. Rottinghaus, H. J. Ju, D. L. Dawe, and E. E. Hiatt. 2001. Ergot alkaloid transport across ruminant gastric tissues. J. Anim. Sci. 79: 542-549.

Hill, N. S. 2005. Absorption of ergot alkaloids in the ruminant. In Neotyphodium in Cool-Season Grasses. C. A. Roberts, C. P. West, and D. E. Spiers. Blackwell Publ., Ames, IA.

Horowitz, M. and U. Meiri. 1993. Central and peripheral contributions to control of heart rate during heat acclimation. Europ. J. Physiol. 422: 386-392.

Horowitz, M. 1998. Do cellular heat acclimation responses modulate central thermoregulatory activity? News Physiol. Sci. 13: 218-225.

Hoveland, C. S. 1993. Economic importance of Acremonium endophytes. Agric. Ecosyst. Environ. 44: 3-12.

Hubbard, R.W., W. T. Matthew, and R. Francesconi. 1982. Heat-stressed rats: Effects of atropine, desalivation, or restraint. J. Appl. Physiol. 53: 1171-1174. 
Hutchinson, J. C., and G. D. Brown. 1969. Penetrance of cattle coats by radiation. J. Appl. Physiol. 26:454-464.

Ishikawa, Y. and O. Hayaishi. 1982. Role of prostaglandin D2 in the hypothermia of rats caused by bacterial lipopolysaccharide. Proc. Natl. Acad. Sci. 79: 6093-6097.

Izon, D.J, J.A. Punt, and W.S. Pear. 2002. Deciphering the role of notch signaling in lymphopoiesis. Curr. Opin. Immunol. 14: 192-199.

Janeway, C. A. Jr.1992. The T-cell receptor as a multicomponent signalling machine: CD4/CD8 coreceptors and CD45 in T-cell activation. Annu. Rev.Immunol. 10: 645-674.

Janeway, C. A. 2007. Janeway's Immunobiology, $7^{\text {th }}$ Ed . Garland science publishing. Arbindon. UK.

Johnson, K. G. and M. Cabanac. 1982. Homeostatic competition between food intake and temperature regulation in rats. Physiol. Behav. 28: 675-679.

Johnson, K. G. and R. Stack. 1989. Adaptive behavior of laboratory rats feeding in hot conditions. Comp. Biochem. Physiol. 94: 69-72.

Jones, K. L., S. S. King, K. E. Griswold, D. Cazac, and D. L. Cross. 2003.

Domperidone can ameliorate deleterious reproductive effects and reduced weight gain associated with fescue toxicosis in heifers. J. Anim. Sci. 81: 2568-2574.

Jones, K. L., S. S. King, and M. J. Iqbal. 2004. Endophyte-infected tall fescue diet alters gene expression in heifer luteal tissue as revealed by interspecies microarray analysis. Mol. Repro. Dev. 67: 154-161.

Kaplan, M.M. and R.D. Utiger. 1978. Iodothyroninemetabolism in liver and kidney homogenates from hyperthyroid and hyothyroid rats. Endocrinology 103: $156-161$.

Kasting, N. W., M. P. Wilkinson, L. D. Lytle, D. W. Taam, and R. J. Wurtman. 1973. Blockade of endotoxin-induced hypothermia by pretreatment with intracisterna endotoxin-induced hypothermia by pretreatment with intracisternal 6-hydroxydopamine. Life Sci. 17: 485-492.

Kasting, N. W. and J. B. Martin. 1983. Changes in immunoreactive vasopressin concentrations in brain regions of the rat in response to endotoxin. Brain Res. 258: $127-132$.

Kent, S., R.M. Bluthe, K.W. Kelley, and R. Dantzer. 1992.Sickness behavior as a new target for drug development. Trends Pharmacol. Sci. 13: 24-28.

Kim, S. and W.M. Yokoyama. 1998. NK cell granule exocytosis and cytokine production inhibited by Ly49A engagement. Cell. Immunol. 183: 106-112. 
Klir, J. J., J. Roth. Z. Szelenyi, J. L. McClellan, and M. J. Kluger. 1993. Role of hypothalamic interleukin- 6 and Tumor necrosis factor- $\alpha$ in LPS fever in rats. Am. J. Physiol. Regulatory Integrative Comp. Physiol. 265: 512-517.

Klotz, J. L., L. P. Bush, D. L. Smith, W. D. Shafer, L. L. Smith, A. C. Vevoda, A. M. Craig, B. C. Arrington, and J. R. Strickland. 2006. Assessment of vasoconstrictiveprotential of D-lysergic acid using an isolated bovine lateral saphenous vein bioassay. J. Anim. Sci. 84: 3167-3175.

Kluger, M.J., K. Rudolph, D. Soszynski, C.A. Conn, L. R. Leon, W. Kozak, E. S. Wallen and P. L Moseley. 1997. Effect of heat stress on LPS-induced fever and tumor necrosis factor. Am. J. Physiol. Regul. Integr. Comp. Physiol. 273: R858R863.

Knochel, J.P. and G. Reed. 1994. Disorders of heat regulation. In: Narins RG, Ed. Maxwell \& Kleeman's Clinical Disorders of Fluid and Electrolyte Metabolism.5th Ed. New York: McGraw-Hill. :1549-1590.

Kondo, M., I.L. Weissman, and K. Akashi. 1997: Identification of clonogenic common lymphoid progenitors in mouse bone marrow. Cell 91: 661-672.

Larson, B. T. 1993. D2 dopamine receptors in fescue toxicosis. Ph.D. Dissertation. Univ. of Missouri, Columbia.

Larson, B. T., D. L. Harmon, E. L. Piper, L. M. Griffis, and L. P. Bush. 1999. Alkaloid binding and activation of D2 dopamine receptors in cell culture. J. Anim. Sci. 77: 942-947.

Lanier, L.L., H. Spits, and J.H. Phillips. 1992. The developmental relationship between NK cells andT cells. Immunol. Today 13: 392-395.

Lin, M.T., H.H. Liu, and Y.L. Yang. 1997. Involvement of interleukin-1 receptor mechanisms in development of arterial hypotension in rat heatstroke. Am. J. Physiol. 273: H2072-H2077.

Lipham, L.B., F. N. Thompson, J. A. Steudemann, and J. L. Sartin. 1989. Effects of metoclopramide on steers grazing endophyte-infected fescue. J. Anim. Sci. 67: 1090-1097.

Luker, F. I., D. Mitchell, and H. P. Laburn. 2000. Fever and motor activity in rats following day and night injections of Staphylococcus aureus cell walls. Am. J. Physiol. Reg. Integ. Comp. Physiol. 279: 610-616.

Lylte L.D., D.W.Taam, and R.J. Wurtman. 1973. Blockade of endotoxin-induced hypothermia by pretreatment with intracisternal6- hydroxydopamine. Life Sci. 13: 485-492.

Maia, A. S. C., R. G. DaSilva, and C. M. Battiston Loureiro. 2005. Sensible and latent heat loss from the body surface of Holstein cows in a tropical environment. Int. J. Biomet. 50: 17-22. 
Malinowski, D. P., D. P. Belesky, and G. C. Lewis. 2005. Abiotic stresses in endophytic grasses. In Neotyphodium in Cool-Season Grasses. C. A. Roberts, C. P. West, and D. E. Spiers. Blackwell Publ., Ames, IA.

Manz, R.A., A. Thiel, and A. Radbruch. 1997. Lifetime of plasma cells in the bone marrow. Nature 388: 133-134.

Masoro, E.J. 2000. Caloric restriction and aging: An update. Exp. Gerontol. 35: 299305.

Masoro, E.J. and S.N. Austad. 1996. The evolution of the antiaging action of dietary restriction: a hypothesis. J. Gerontol., Ser. A, Biol. Sci. Med. Sci. 51A: B387-B391.

Matthew, C. B. 1997. Telemetry augments the validity of the rat as a model for heat acclimation. Annals New York Acad. Sci. 813: 233-238.

Meerlo, P., S. F. De Boer, J. M. Koolhaas, S. Daan, and R. H. Van Den Hoofdakker. 1996. Changes in daily rhythms of body temperature and activity after a single social defeat in rats. Physiol. Behav. 59:735-739.

Mercer, J. 2001. Glossary of terms for thermal physiology. Jpn. J. Physiol. 51: 245280.

Miyake, K. 2007 Innate immune sensing of pathogens and danger signals by cell surface Toll-like receptors. Semin Immunol. 19: 3-10.

Mizinga, K. M., F. N. Thompson, J. A. Stuedemann, and G. L. Edwards. 1993. Neural dopamine D2 receptors in rats fed endophyte-infected fescue seed. Drug Chem. Toxicol. 16: 307-319.

Mosmann, T. and Coffman, R. 1989. Th1 and Th2 cells: Different patterns of lymphokine secretion leads to different functional properties. Annu. Rev. Immunol. 7, 145-173

Munch, I. C. 1995. Influences of time intervals between meals and total food intake on resting metabolic rate in rats. Acta Physiol. Scand. 153: 243-247.

Muthukumar, A.R., C.A. Jolly., K. Zaman, and G. Fernandes. 2000.

Calorie restriction decreases proinflammatory cytokines and polymeric Ig receptor expression in the submandibular glands of autoimmune prone NZB(NZW)F1 mice. J. Clin. Immunol. 20: 354-361.

Neal, W. D. and S. P. Schmidt. 1985. Effects of feeding Kentucky 31 tall fescue seed infected with Acremonium coenophialum to laboratory rats. J. Anim. Sci.61:603-611.

Ochsenbein, A.F., D.D. Pinschewer, S. Sierro,E. Horvath, H. Hengartner, and R.M. Zinkernagel. 2000. Protective long-term antibody memory by antigen-driven and 
T help-dependent differentiation of longlived memory B cells to short-lived plasma cells independent of secondary lymphoid organs. Proc. Natl. Acad. Sci. USA 97: 13263-13268.

Oliver, J.W. 1997. Physiological manifestations of endophyte toxicosis in ruminant and laboratory species. In: C.W. Bacon and N.S. Hill, Ed. Neotyphodium/ Grass Interactions, Plenum Press, NY.

Oliver, J.W., J.R. Strickland, J. C. Waller, H. A. Fribourg, R. D. Linnabary, and L. K.Abney. 1998. Endophytic fungal toxin effects on adrenergic receptors in bovine lateral saphenous veins of cattle grazing tall fescue. J. Anim. Sci. 76: 2853-2856.

Oliver, J.W., A.E. Schultze, B.W. Rohrbach, H.A. Fribourg, T. Ingle, and J. C. Waller. 2000. Alterations in hemograms and serum biochemical analytes of steers after prolonged consumption of endophyte-infected tall fescue. J. Anim. Sci. 78: 1029-1035.

Oliver, J. W. 2005. Pathophysiologic response to endophyte toxins. In Neotyphodium in Cool-Season Grasses. C. A. Roberts, C. P. West, and D. E. Spiers. Blackwell Publ., Ames, IA.

Oppmann, B. 2000. Novel p19 protein engages IL-12p40 to form a cytokine, IL-23, with biological activities similar as well as distinct from IL-12. Immunity 13: $715-725$.

Osborn, T. G., S. P. Schmidt, D. N. Marple, C. H. Rahe, and J. R. Steenstra. 1992. Effect of consuming fungus-infected and fungus-free tall fescue and ergotamine tartrate on selected physiological variables of cattle in environmentally controlled conditions. J. Anim. Sci. 70: 2501-2509.

Pahlavani, M.A. 2004. Influence of caloric restriction on aging immune system. J. Nutr. Health Aging 8: 38- 47.

Panaccione, D. G., R. D. Johnson, J. Wang, C. A. Young, P. Damrongkool, B. Scott, and C. L. Schardl. 2001. Elimination of ergovaline from a grass-Neotyphodium endophyte symbiosis by genetic modification of the endophyte. Proc. Natl. Acad.Sci. USA. 98: 12820-12825.

Pereira, A. M., F. Baccari, E. A., Titto, and J. A. Almeida. 2007. Effect of thermal stress on physiological parameters, feed intake and plasma thyroid hormones concentration in Alentejana, Mertolenga, Frisian and Limousine cattle breeds. Int. J. Biometeorol. 52. 199- 208.

Poetschke H.L., D.B.Klug, S.N. Perkins, T.Thomas.Y. Wang, E.R. Richie, and S.D. Hursting. 2000. Effects of calorie restriction on thymocyte growth, death and maturation. Carcinogenesis. 21: 1959-1964.

Pollock, J.D. and N. Rowland. 1981. Peripherally administered serotonin decreases food intake in rats. Pharmacol. Biochem. Behav. 15: 179-183. 
Porter J.K. 1995. Analysis of endophyte toxins: fescue and other grasses toxic to livestock, J. Anim. Sci.75: 871-880.

Prasad, M., G. Uma Devi, and S. Govindappa. 1989. Effect of hypo and hyperprolactinemia on the testicular maturation of Wistar rats during pubertal transition. Arch. Int. Physiol. Biochem. 97: 347-353.

Raetz C. R. H and C. Whitfield. 2002. Lipopolysaccharide endotoxins. Ann. Rev. Biochem. 71: 635-700.

Reber, P.M. 1993. Prolactin and immunomodulation. Am. J. Med. 95: 637-64.

Rhodes, M. T., J. A. Paterson, M. S. Kerley, H. E. Garner, and M. H. Laughlin. 1991. Reduced blood flow to peripheral and core body tissues in sheep and cattle induced by endophyte-infected tall fescue. J. Anim. Sci. 69: 2033-2043.

Rice, R.L., D.J. Blodgett, G. Schurig, W.S. Swecker, J.P. Fontenot, V.G. Allen, and R. M. Akers. 1997. Evaluation of humoral immune responses in cattle grazing endophyte-infected or endophyte-free fescue. Vet. Immunol. Immunopathol. 59: 285-291.

Romanovsky, A. A., O. Shido, S, S. Sakurada, N. Sugimot, and T. Nagasaka. 1996.Endotoxin shock: Thermoregulatory mechanisms. Am. J. Physiol. Regul. Integr.Comp. Physiol. 270: 693-703.

Roberts, C. A., D. E. Spiers, A. L. Karr, H. R. Benedict, D. A. Sleper, P. A. Eichen, C. P. West, E. L. Piper, and G. E. Rottinghaus. 2002. Use of a rat model to evaluate tall fescue seed infected with introduced strains of Neotyphodium coenophialum. J. Agric. Food Chem. 50: 5742-5745.

Rottinghaus, G. E., L. M. Schultz, P. F. Ross, and N. S. Hill. 1993. An HPLC method for the detection of ergot in ground and pelleted feeds. J. Vet. Diagn. Invest. 5: $242-247$.

Saker, K. E., V. G. Allen, J. Kalnitsky, C. D. Thatcher, W. S. Swecker, Jr., and J. P. Fontenot. 1998. Monocyte immune cell response and copper status in beef steers that grazed endophyte-infected tall fescue. J. Anim. Sci. 76: 2694-2700.

Saker, K. E., V. G. Allen, J. P. Fontenot, C. P. Bagley, R. L. Ivy, R. R. Evans and D. B Wester. 2001. Tasco-Forage: II. Monocyte immune cell response and performance of beef steers grazing tall fescue treated with a seaweed extract. J.Anim.Sci. 79: 1022-1031.

Santoli, D., Trinchieri G, Koprowski H. 1978. Cell-mediated cytotoxicity against virus-infected target cells in humans. II. Interferon induction and activation of natural killer cells. J. Immunol. 81: 1010-1021.

Saper, C.B. and C.D. Breder. 1994. The neurologic basis of fever. N. Engl. J. Med. 330: 1880-1884. 
Scharton T.M. and P. Scott. 1993. Natural killer cells are a source of interferon that drives differentiation of CD4C $\mathrm{T}$ cell subsets and induces early resistance to Leishmania majorin mice. J. Exp. Med. 178: 567-577.

Schmidek, W. R., E. E. Zachariassen, and H. T. Hammel. 1983. Total calorimetric measurements in the rat: Influence of the sleep-wakefulness cycle and of the environmental temperature. Brain Res. 288: 261-271.

Schmidt-Nielsen, K. and B. Schmidt-Nielsen. 1952. Water metabolism of desert mammals. Physiol. Rev. 32: 135-166.

Schoning, C., M. Flieger, and H. H. Pertz. 2001. Complex interaction of ergovaline with5-HT2A, 5-HT1B/1D, and alpha 1 receptors in isolated arteries of rat and guinea pig. J. Anim. Sci. 79: 2202-2209

Schmidt, S. P., C. S. Hoveland, E. M. Clark, N. D. Davis, L. A. Smith, H. W. Grimes, and J.L.Holliman. 1982. Association of an endophytic fungus with fescue toxicity in steers fed Kentucky 31 tall fescue seed or hay. J. Anim. Sci. 50: 1259 1269.

Scales, W. E. and M. J. Kluger. 1987. Effect of antipyretic drugs on circadian rhythm in body temperature of rats. Am. J. Physiol. Regul. Int. Comp. Physiol. 253: R306-313.

Settivari, R. S., Bhusari, T. Evans, P.A. Eichen, L.B. Hearne, E. Antoniou, and D.E. Spiers. 2006. Genomic analysis of the impact of fescue toxicosis on hepatic function, J. Anim. Sci. 84: 1279-1294.

Settivari, R.S., T.J. Evans, P.A. Eichen, G.E. Rottinghaus, and D.E. Spiers. 2008. Short - and long-term responses to fescue toxicosis at different ambient temperatures. J. Therm. Biol 33: 213-222.

Severinsen, T. and I. C. Munch. 2001. Body core temperature during food restriction in rats. Acta Physiol. Scand. 165: 299-305.

Shen J., H. Ren, C. Tomiyama- Miyaji, M. Watanable, E. Kainuma, M. Inoue, Y. Kuwano, and T. Abo. 2009. Resistance and augmentation of innate immunity in mice exposed to starvation. Cell Immunol. 259: 66-73.

Shibolet, O., R. Alper, Y. Avraham, E. M. Berry, and Y. Ilan, Y. 2002. Immunomodulation of experimental colitis via caloric restriction: Role of Nk1.1+ T cells. Clin. Immunol. 105: 48 - 56.

Shido, O., S. Sakurada, and T. Nagasaka. 1991. Effect of heat acclimation on diurnal changes in body temperature and locomotor activity in rats. J. Physiol. 433: 5971.Silanikove, N. 2000. Effects of heat stress on the welfare of extensively managed domestic ruminants. Livestock Prod. Sci. 67: 1-18

Sims, J.E., J.G. Giri, and S.K. Dower. 1994. The two interleukin-1 receptors play different roles in IL-1 activities. Clin. Immunol. Immunopathol. 72: 9-14. 
Sleper, D. A. and R. C. Buckner. 1995. The fescues. In: Forages, vol. I: An introduction to grassland agriculture. R. F. Barnes, D. A. Miller and C. J. Nelson Ed. Iowa State University Press, Ames, IA.

Smith, V. G., R. R. Hacker, and G. Brown. 1997. Effect of alterations in ambient temperature on serum prolactin concentration in steers. J. Anim. Sci. 44: 645654.

Spiers, D. E., Q. Zhang, P. A. Eichen, G. E. Rottinghaus, G. B. Garner, and M. R. Ellersieck. 1995. Temperature-dependent responses of rats to ergovaline derived from endophyte-infected tall fescue. J. Anim. Sci.73: 1954-1961.

Spiers, D.E., P.A. Eichen, and G.E. Rottinghaus. 2005. A model of fescue toxicosis: responses of rats to intake of endophyte-infected tall fescue, J. Anim. Sci. 83: $1423-1434$.

Steele, R. G. and J. H. Torrie. 1980. Principles and Procedures for Statistics: A Biometric Approach. McGraw Hill, New York, NY.

St-Pierre, N. R., B. Cobanov, and G. Schnitkey. 2003. Economic losses from heat stress by US livestock industries. J. Dairy Sci. 86: E52-77.

Strickland, J. R., J. W. Oliver, and D. L. Cross. 1993. Fescue toxicosis and its impact on animal agriculture. Vet. Human Tox. 35:454-464.

Stuedemann, J. A. and D. H. Seman. 2005. Integrating genetics, environment and management to minimize animal toxicosis. In Neotyphodium in Cool-Season Grasses. C. A. Roberts, C. P. West, and D. E. Spiers. Blackwell Publ., Ames, IA.

Taylor, J.M.G., J.L. Fahey, R. Detels, and J.V. Giorgi. 1989. CD4 percentage, CD4 number, and CD4/CD8 ratio in HIV infection: Which to choose and how to use. J. Acquir. Immune Defic. Syndr. 2: 114-124.

Thompson, F. N. and J. A. Stuedemann. 1993. Pathophysiology of fescue toxicosis. In: R. Joost and S. Quissenberry Eds. Acremonium/Grass interaction.Elseview, The Netherlands.

Thompson, F. N., J. A. Stuedemann, and N. S. Hill. 2001. Anti-quality factors associated with alkaloids in eastern temperature pasture, J. Range Manag. $54: 474-489$.

Tian, L., Q. Cai, R. Bowen, and H. Wei. 1995. Effects of caloric restriction on age-related oxidative modifications of macromolecules and lymphocyte proliferation in rats. Free Radic. Biol. Med. 19: 859- 865.

Tracey, K.J., B. Beutler, and S.F. Lowry. 1986. Shock and tissue injury induced by recombinant human cachectin. Science 234: 470-474. 
Tracey, K.J., S. F. Lowry, T.J. Fahey .1987. Cachectin/tumor necrosis factor induces lethal shock and stress hormone responsesin the dog. Surg. Gynecol. Obstet. 164: 415-422.

Trinchieri, G., D. Santoli, and H. Koprowski. 1978. Spontanteous cell-mediated cytotoxicity in humans: role of interferonand immunoglobulins. J. Immunol. 120: 1849-1855.

Trinchieri, G. 1989. Biology of natural killer cells. Adv. Immunol. 47: 187-376.

. Van Damme, J., G. Opdenakker, and R.J. Simpson. 1987. Identification of the human $26-\mathrm{kD}$ protein, interferon b2 (IFN-b2) as a B-cell hybridoma/ plasmacytoma growth factor induced by interleukin-1 and tumor necrosis factor. J. Exp. Med. 165. 914-919.

Varney, D.R., L.A. Varney, P.M. Zavos, R.W. Hemken, M.D. Wiglesworth, and M.R.Siegel. 1991. Tall fescue endophyte: effect on congenital development and pup growth in mice. J. Dairy Sci. 74: 460-465.

Wallace, L. L., D. W. Vogt, R. J. Lipsey, G. B. Garner, and C. N. Cornell. 1996. Effects of environmental heat and intake of tall fescue seed infested with Acremonium coenophialum on the acid-base status of young bulls. J. Vet. Diag. Invest. 8: 233-237.

Wanagat, J., D.B Allison, and R. Weindruc. 1999. Caloric intake and aging: mechanisms in rodents and a study in nonhuman primates. Toxicol. Sci. 52: 540 (suppl.).

Weil, Z.M., S.L. Bowers, L.M. Pyter, and R.J. Nelson. 2006. Social interactions alter proinflammatory cytokine gene expression and behavior following endotoxin administration. Brain Behav.Immun.. 20: 72-79.

Wellman, P. J., B. T. Davies, and A. Morien. 1993. Modulation of feeding by hypothalamic paraventrucular nucleus alpha 1- and alpha 2 adrenergic receptors. Life Sci. 53: 669-679.

West, J. W. 2003. Effects of heat-stress on production in dairy cattle. J. Dairy Sci. 86: $2131-2144$

Wright, S.D. 1995. CD14 and innate recognition of bacteria. J. Immunol. 155: 6-8.|

Wu, C. 1995. Heat shock transcription factors: structure and regulation. Annul. Rev. Cell Dev. Boil. 11: 441-469.

Yoda, T. C., L. I. Yoshida, K. Su, L. Hosono, T. Shido, O. Sakurada, S. Fukuda, Y. Kanosue, K. Kazuyuki. 2000. Effects of food 61 deprivation on daily changes in body temperature and behavioral thermoregulation in rats. Am. J. Physiol. Regul. Integ. Comp. Physiol. 278: R134-139. 
Yokoyama, W.M. 1998. Natural killer cell receptors. Curr. Opin. Immunol. 10: 298 305.

Yousef, M.K. 1985. Stress Physiology in Livestock. BasicPrinciples, Vol. 1. CRC Press, Boca Raton, FL.

Zanzalari, K.p., R. N. Heitmann, J.B. McLaren, and H.A. Fribourg. 1989.Effects of endophyte-infected fescue and cimetidine on respiration rates, rectaltemperaturesand hepatic mixed function oxidase activity as measured by hepatic antipyrinemetabolism in sheep. J. Anim. Sci. 67: 3370-3378.

Zetterstrom, M., A.K. Sundgren-Andersson, P. Ostlund, and T. Bartfai. 1998. Delineation of the proinflammatory cytokine cascade in fever induction. Ann. NY Acad. Sci. 856: 48-52.

Zhang, Q., D. E. Spiers, G. E. Rottinghaus, and G. B. Garner. 1994. Thermoregulatory effects of ergovaline isolated from endophyte-infected tall fescue seed on rats. J. Ag. Food Chem. 42: 954-958.

Zuckerman, S.H. and S. Butter. 1989. Differential regulation of lipopolysaccharide-induced interleukin 1 and tumor necrosis factor synthesis: Effects of endogenous and exogenous glucocorticoids and the role of the pituitary-adrenal axis. Eur. J. Immunol. 19: 301-305. 


\section{Appendix 1}

\section{Implanting Minimitters in rats}

Surgical instruments were autoclaved.Temperature transponders were sterilized using zephiran chloride and rinsed with sterile saline prior to use. Animals will be anesthetized with isoflurane or rat cocktail (83mg/ml Ketamine/ml Xylazine@ $0.1 \mathrm{ml} / 100 \mathrm{gBW}, \mathrm{IM}$ ) and hair will be clipped from the incision site (ventral midline or abdominal flank) and removed with topical application of depilatory cream and site cleaned with alcohol and betadine three times. Ointment in the eyes is not needed as its only a short procedure.

The incision was made through the ventral midline just large enough to insert the transponder, approximately $12 \mathrm{~mm}$. Then transponder was sutured to the body wall to prevent migration in to the inguinal canal. The peritoneum was closed with Vedco Supra Sorb absorbable 4-0 suture and skin closed with Vedco Supra Sorb absorbable 4-0 suture using simple interrupted pattern.

Following surgical placement of transmitters, rats recieved Buprenex (Buprenorphine- 0.1 to $0.05 \mathrm{mg} / \mathrm{kg}$ ) subcutaneously. Additional Buprenex can be adiministered if necessary. All animals were kept warm on heating pads and monitored for return to sternal recumbency then moved to housing cages where core body temperature and activity were monitored continuously. Animals will not be tested for the first week after surgery. 


\section{Appendix 2}

\section{Materials}

\section{Materials:}

1. DPBS (Dulbcecco's phosphate buffered saline ) Cat no \# 1760420

2. RPMI- Invitrogen Cat no \# 22400-089

3. Histopaque- Sigma Aldrich Cat no \# 10771

4. Pipette tips (fischer brand)- 1-200 $\mu 1$ Cat no \#21-197-8k ,0.5-1 $\mu 1$ Cat no\# 02-581-5.

5. $1000 \mu 1$ Cat no \# 21-197-8s.

6. Microiltre tubes- Fisher brand Cat no \# 502GRD-SC-FCS

7. Fc block (rat)- BD biosciences- Cat no- 550270

8. NK/B/T cocktail- BD biosciences- Cat no- 558495

9. ELISA Kits TNF $\alpha$ R\&D Systems Cat no \# RTA00

10. IL1 $\beta$ cat no \# R\&D Systems Cat no \# RLB00

11. Pipettes and LPS material

12. Syringes and needles for blood collection

13. Heparinised tubes

14. Polystyrene tubes for flow - BD falcon Fischer Cat no \# 352052

15. Cell strainer for flow BD falcon Fischer Cat no \# 08771-23

16. Microscope or cell counter

17. Mcmaster slide and cover slips

18. APC control - anti - rat CD4 - e biosciences Cat no \# 17-0040-80

19. PE control - anti - rat CD8 - e biosciences Cat no \# 12-0084-80

20. FITC control - anti-rat CD 90 - e biosciences Cat no \# 11-0900-81 


\section{Isolation of rat peripheral blood lymphocytes (PBL)}

Add room temperature PBS to the tube containing heparized or EDTA whole rat blood to make a $50 \%$ blood-50\%PBS mixture, then mix gently by inversion.

Carefully overlay 4 to $7 \mathrm{~mL}$ of diluted blood onto $3 \mathrm{~mL}$ of room temperature FicollHypaque $(\mathrm{d}=1.007)$ in a $15 \mathrm{ml}$ conical bottom centrifuge tube. (It is critical that the blood, PBS, and Ficoll be at room temperature. Be very slow and don't pipette very fast)

Centrifuge at $1500 \mathrm{rpm}(300 \mathrm{X} \mathrm{g})$ at $25^{\circ} \mathrm{C}$ for 40 mins (Program 3 - Dr. F. centrifuge) The red blood cells should pellet at the bottom of the tube and the lymphocytes should be seen as a whitish band at the interface. (Press bulb outside before inserting transfer pipette)

Carefully remove the tubes from the centrifuge and using a plastic disposable pipette collect the lymphocytes from the interface. Try to collect no more than 1-1.5 mls.

Place the lymphocytes in a 15-ml conical centrifuge tube and dilute with $\sim 13 \mathrm{mls}$ of ice-cold RPMI $+10 \%$ FBS.

Centrifuge at 2200rpm(1000G) for 5 mins. Repeat this washing procedure. 
Resuspend the final pellet in cold RPMI $+10 \%$ FBS using the same volume as the original blood sample that you started with prior to dilution.

Count an aliquot of cells with trypan blue to determine cell number and viability. The cells are now ready to use. Place tubes with cells on ice!

\section{Antibody labeling and Fc Block}

Transfer $1 \times 10^{6} \mathrm{PBL}$ into a $12 * 75 \mathrm{~mm}$ polystyrene test tube (appropriate for flow cytometry -BD Falcon \# 352052), keeping cells on ice.

Centrifuge at 1700rpm (400G) for 5 mins)

Using an aspirator, suck supernatant

Add $2 \mu 1$ /tube CD32 (Fc Block) $+50 \mu 1$ PBS; mix by vortexing vigorously for 5 sec.

Incubate 30 minutes on ice;

Repeat ; Centrifuge at 1700rpm (400G) for 5 mins).Using an aspirator, suck supernatant 
Add $20 \mu 1$ rat cocktail (NK/T/B or CD4/CD8) + $30 \mu 1 \mathrm{PBS}$ (vortex).

Control tubes- $1 \mu 1$ PE anti-CD8 $+50 \mu 1$ PBS (Vortex), $1 \mu 1$ APC Anti-rat CD4 +50 $\mu 1$ PBS (Vortex), $1 \mu 1$ FITC Anti-rat CD 90+50 $\mu 1$ PBS (Vortex),

Incubate 30 minutes on ice (protect from light)

Repeat Centrifuge at 1700rpm (400G) for 5 mins).Using an aspirator, suck supernatant

Add $500 \mu 1$ of PBS in each tube; cap \& vortex; store in refrigerator; protect from light.

Prior to taking sample to flow lab, pass them through a mini-filtration device (cat. \# BD falcon 08771-23) to remove any clumped cells that will clod the flow cytometer. Run samples within 24 hrs. 
Appendix 3

\begin{tabular}{lll} 
Treatment groups & Thermoneutral (n) & Heat stress (n) \\
\hline E-LPS- & 6 & 6 \\
E-LPS+ & 6 & 6 \\
E+LPS- & 6 & 6 \\
E+LPS+ & 6 & 6 \\
PF TO E+ LPS- & 6 & 6 \\
PF TO E+ LPS+ & 6 & 6 \\
\hline
\end{tabular}




\section{Appendix 4}
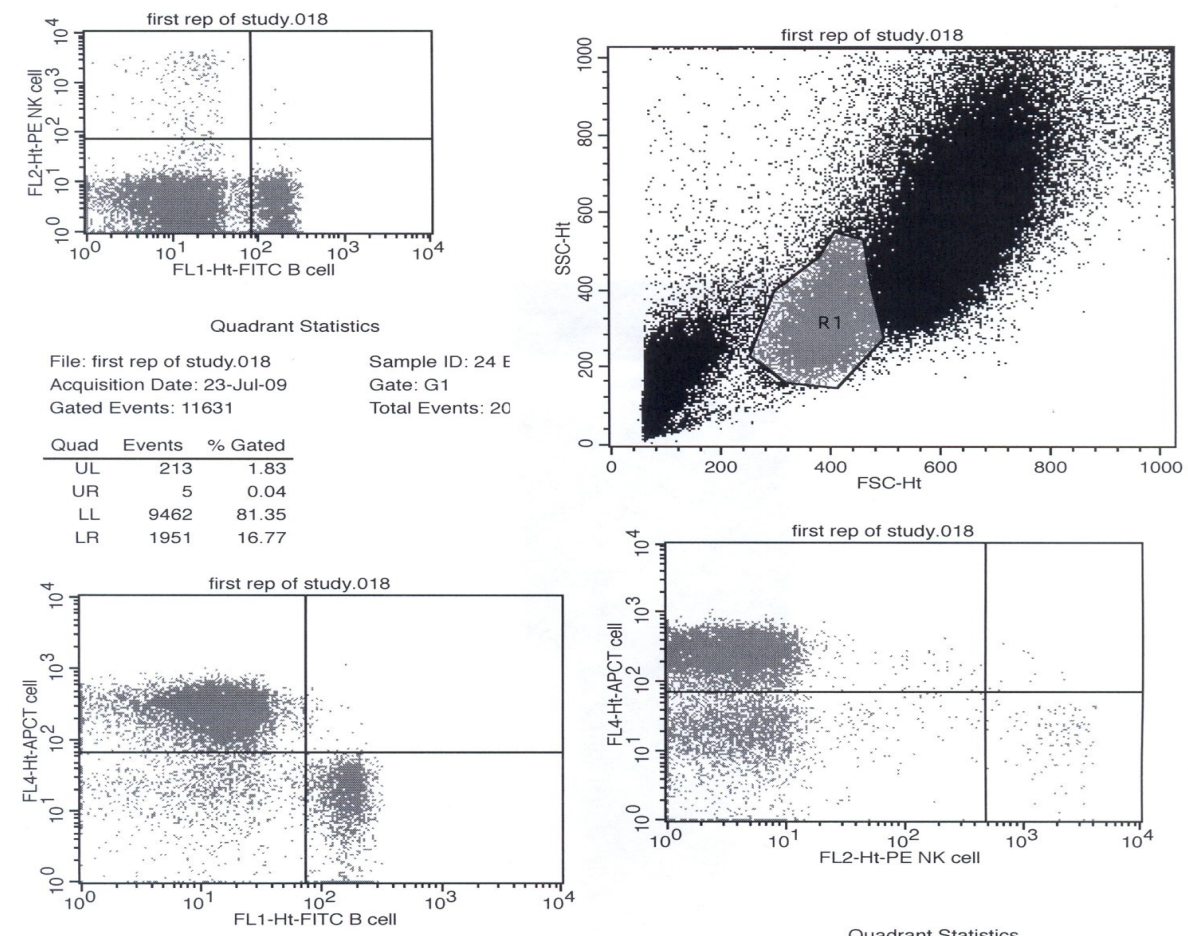

Quadrant Statistics

File: first rep of study. 018

Sample ID: $24 \mathrm{E}+\mathrm{TN}$

Acquisition Date: 23Jul09

Gate: G1

Gated Events: 11631

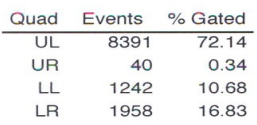

Quadrant Statistics

File: first rep of study. 018

Sample ID: $24 \mathrm{E}+\mathrm{TN}$

Acquisition Date: $23 \mathrm{Jul}-09$

Gate: G1

Gated Events: 11631
Total Events: 204000
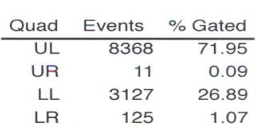

Page 1

Results of flow cytometry were $\mathrm{R} 1$ refers to the gate around lymphocytes. Then samples were separated in to T, B and NK cells using four quadrant model. The figure also shows forward and side scatter and the location of the lymphocytes in relation to forward and side scatter. 
